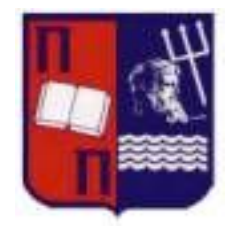

\title{
RISK AND CRISIS MANAGEMENT
}

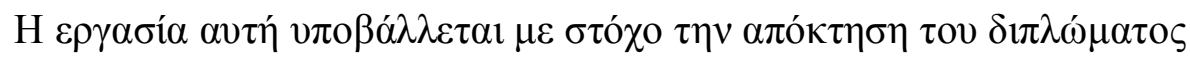

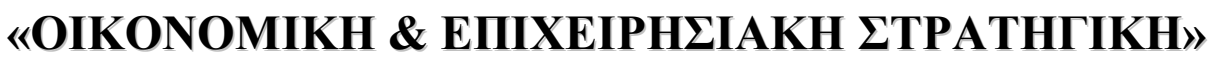

$\alpha \pi$ to

ПАNЕПІ $\Sigma$ THMIO ПЕIPAI $\Omega \Sigma$

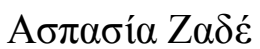

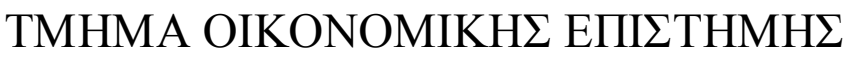




\section{$\Delta \mathbf{H} \Lambda \Omega \Sigma H$}

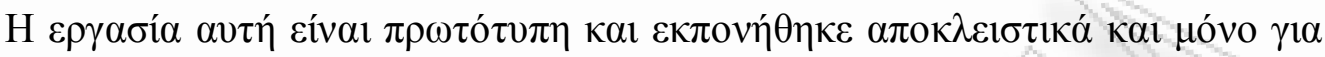

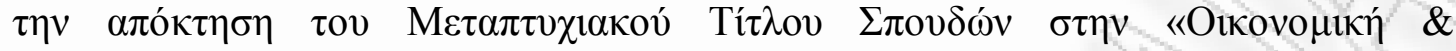

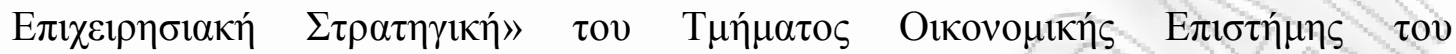

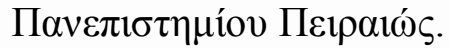

$\mathrm{H} \Delta \eta \lambda \circ v ́ \sigma \alpha$,

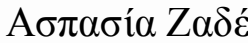




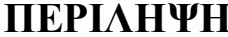

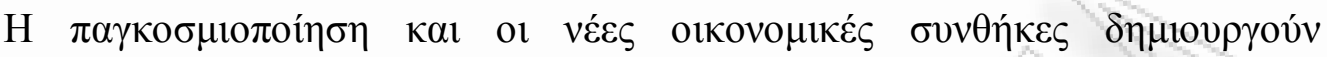

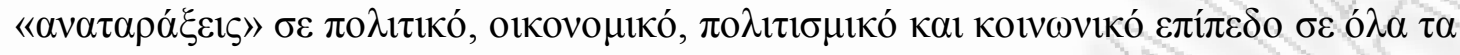

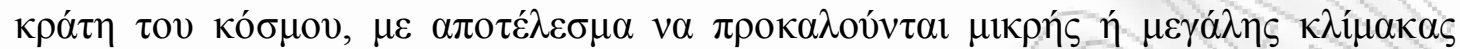

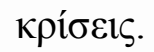

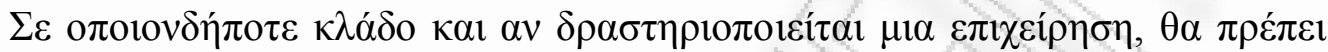

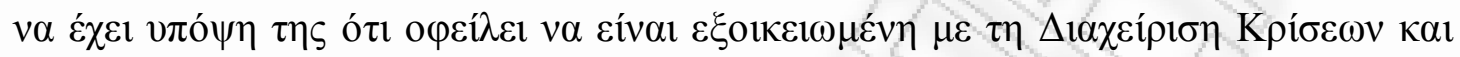

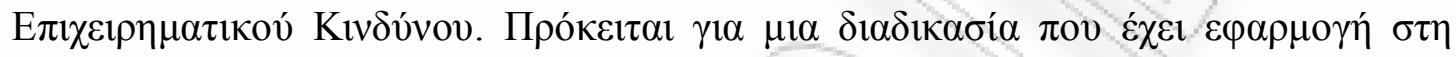

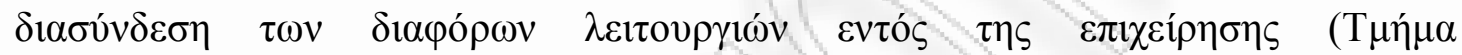

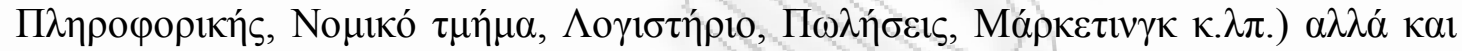

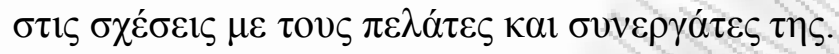

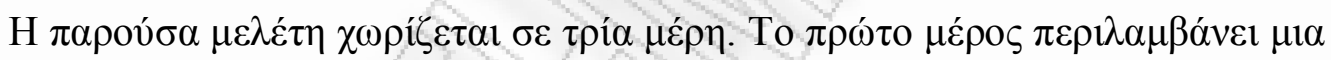

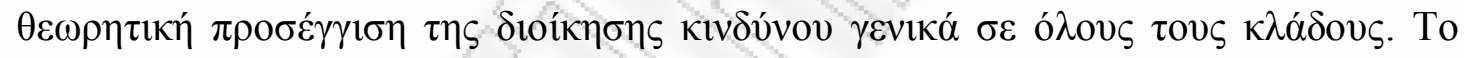

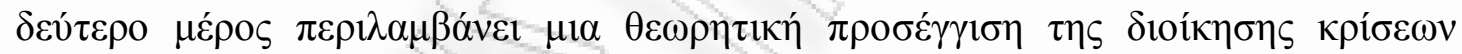

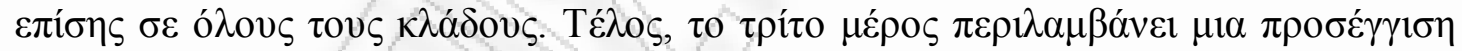

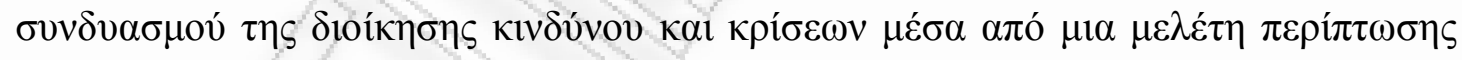

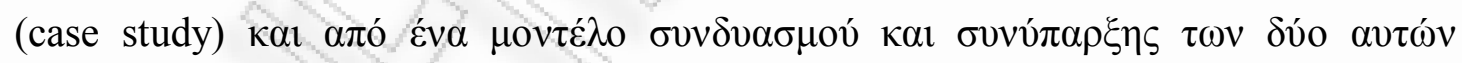

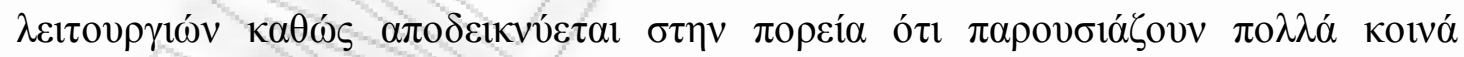
$\sigma \eta \mu \varepsilon^{i} \alpha$. 


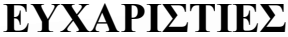

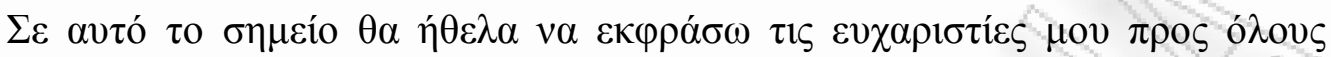

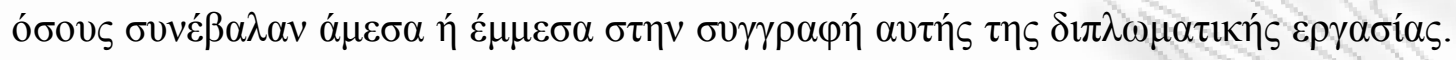

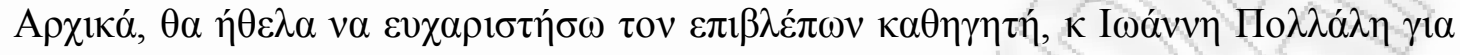

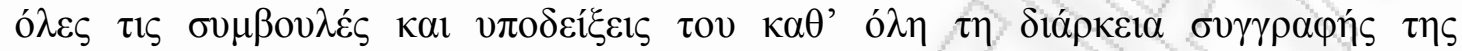

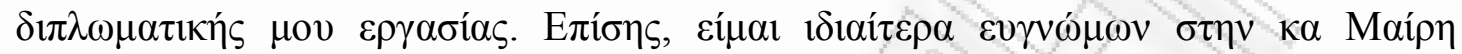

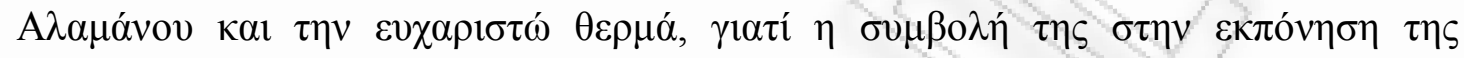

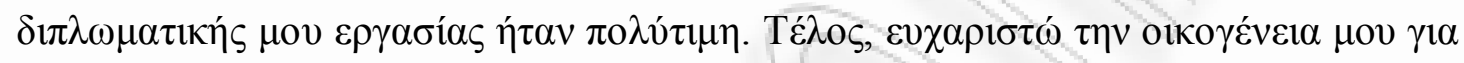

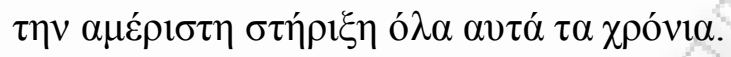




\section{ПEPIEXOMENA}

MEPOE A

\section{$\Delta I O I K H \Sigma H ~ K I N A Y N O Y$ (RISK MANAGEMENT)}

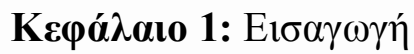

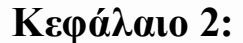

2.1 Opıruoí 8

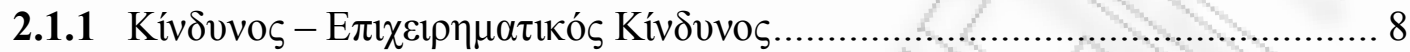

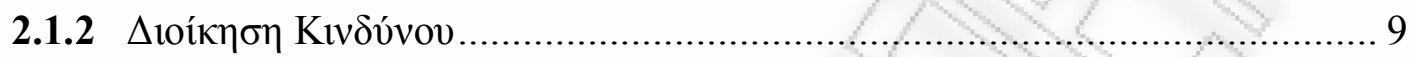

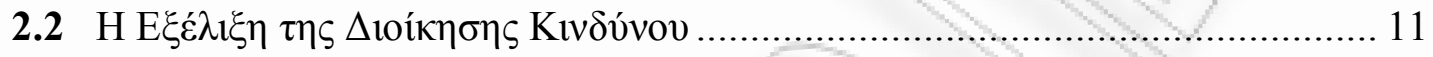

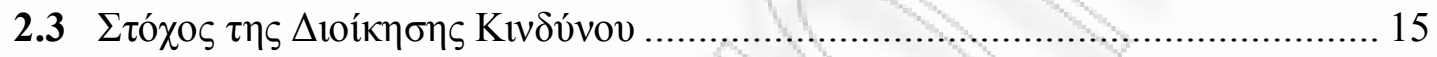

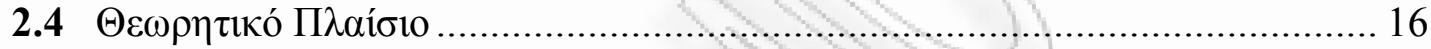

2.4.1 T

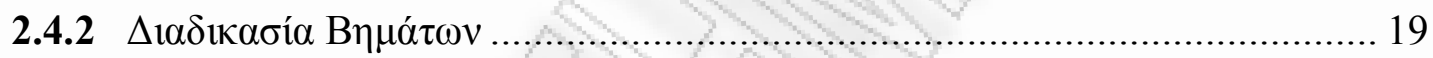

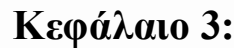

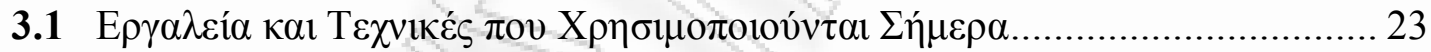

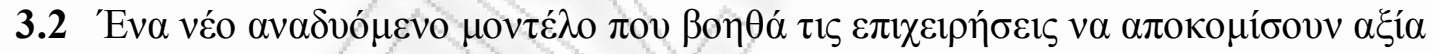

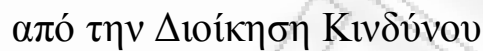
27

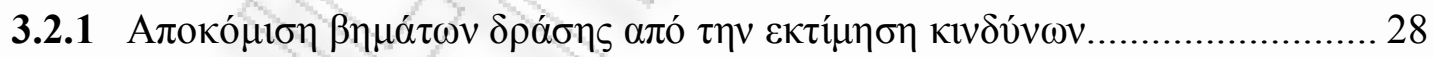

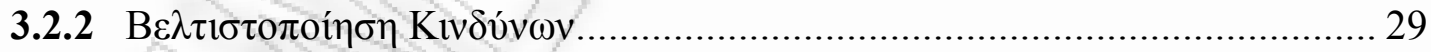

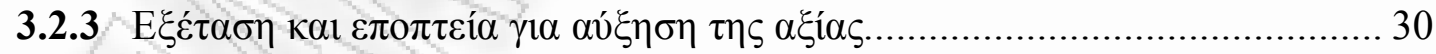

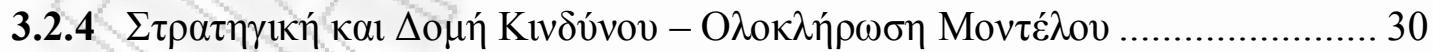

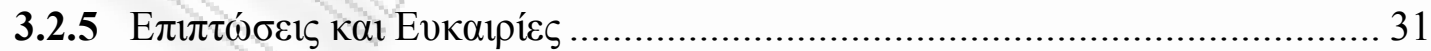

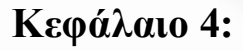

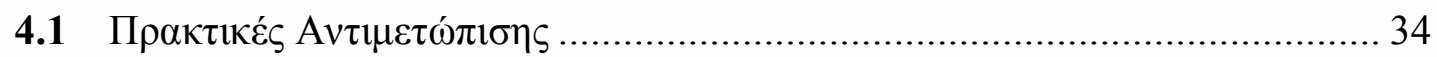

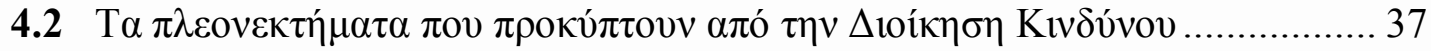




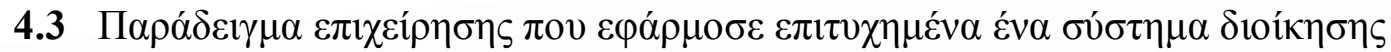

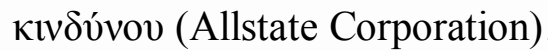

MEPOI 'B

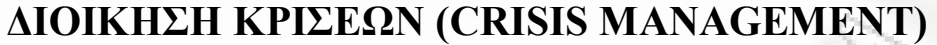

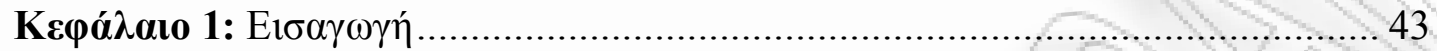

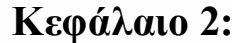

2.1 Opıбноí

2.1.1 Kрíon 44

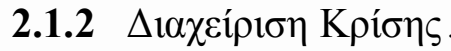
45

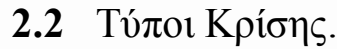
46

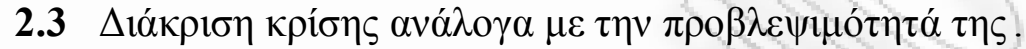
48

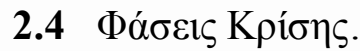
48

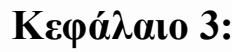

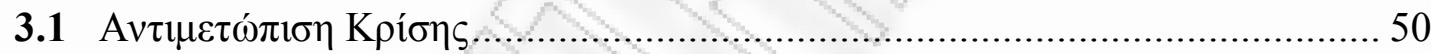

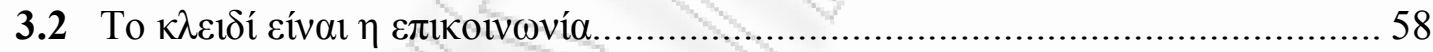

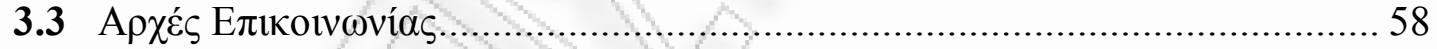

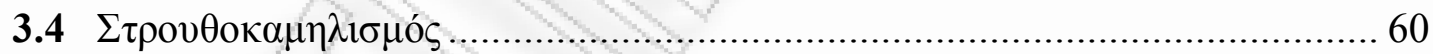

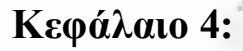

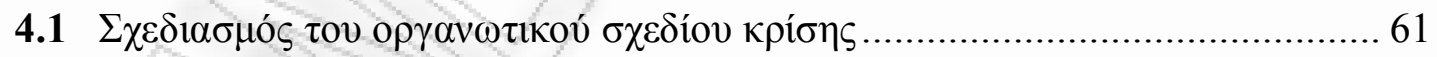

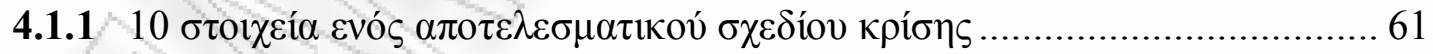

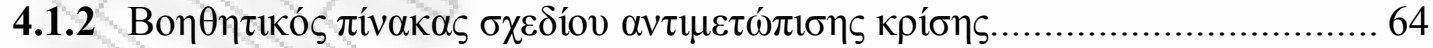

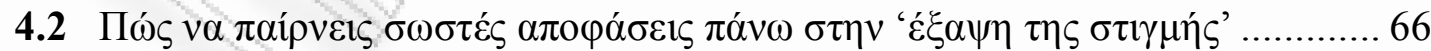




\section{$\operatorname{MEPO\Sigma } \cdot \Gamma$}

\section{RISK \& CRISIS MANAGEMENT: $\Sigma$ YNAYA $\Sigma M O \Sigma$}

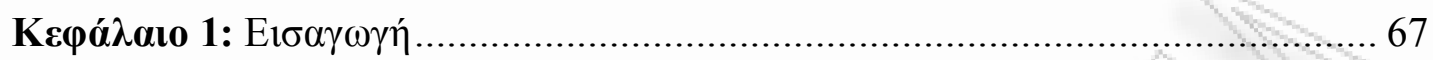

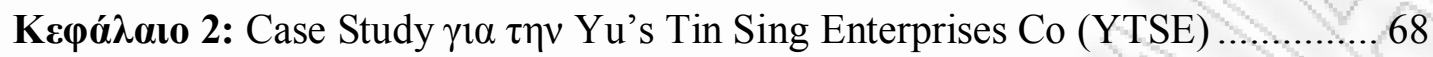

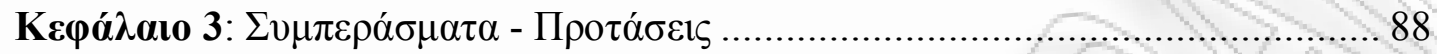

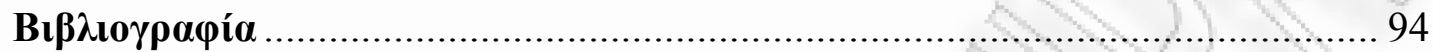




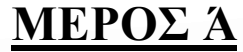

\section{$\Delta I O I K H \Sigma H ~ K I N \Delta Y N O Y$ (RISK MANAGEMENT)}

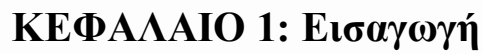

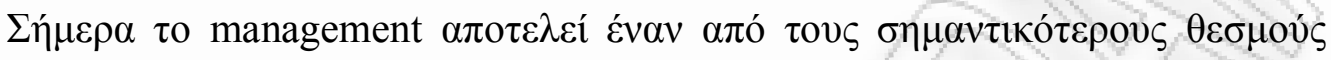

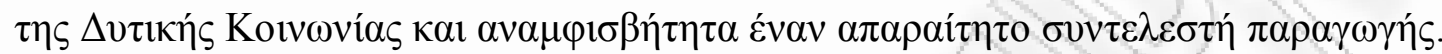

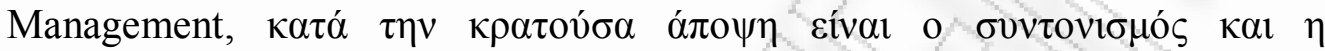

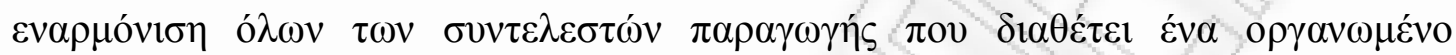

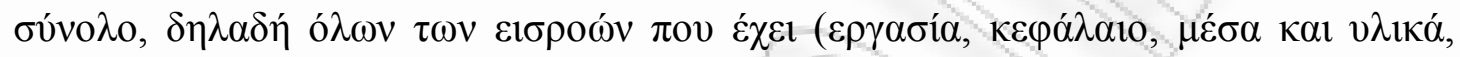

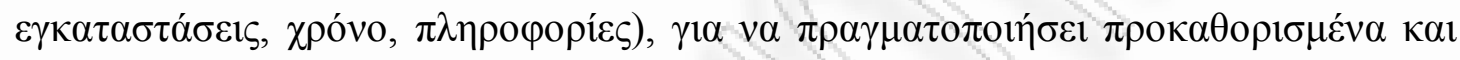

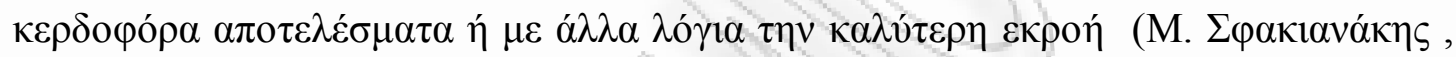
1998).

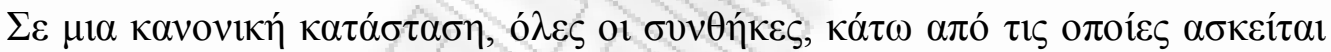

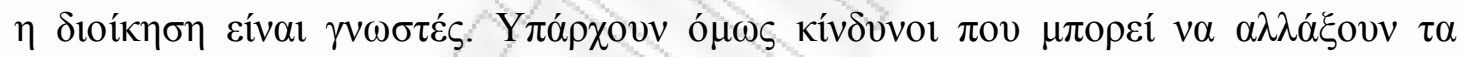

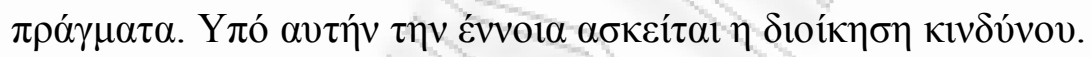

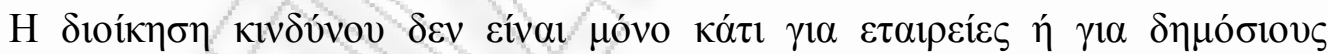

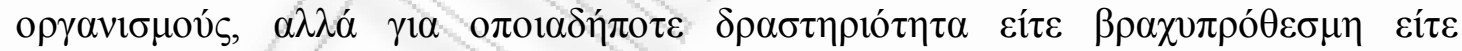

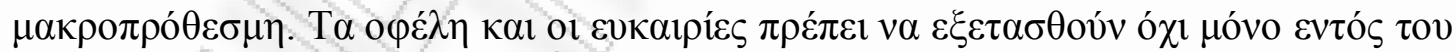

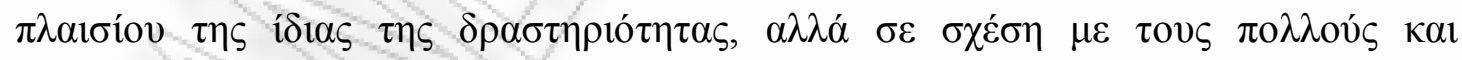

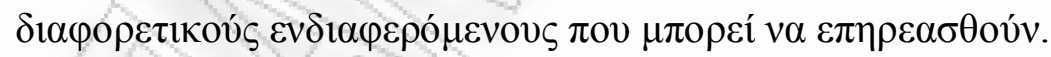




\section{КЕФАААIO 2}

\subsection{Opıбнoí}

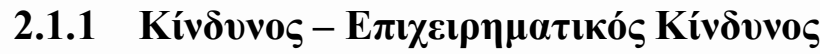

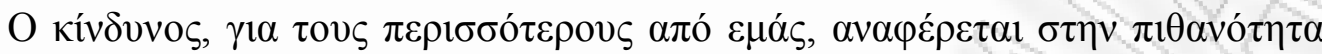

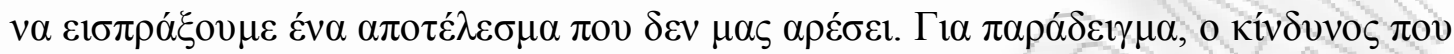

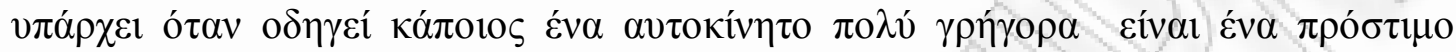

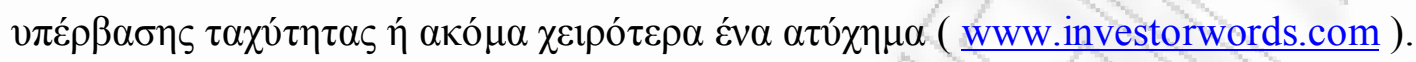

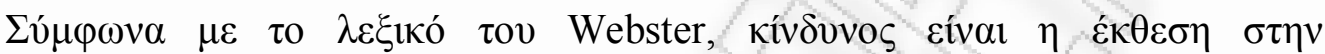

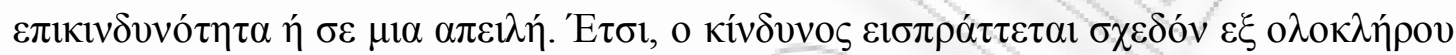
$\mu \varepsilon \alpha \rho v \eta \tau \iota \kappa \eta ́ ~ \varepsilon ́ v v o 1 \alpha$.

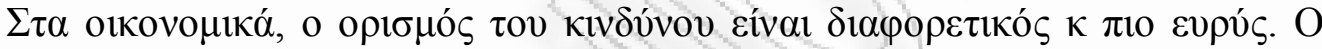

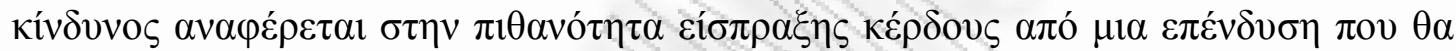

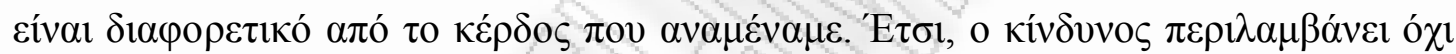

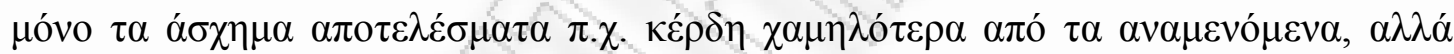

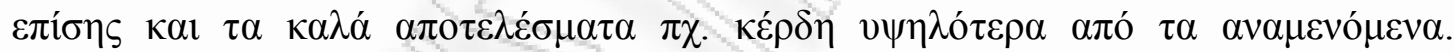

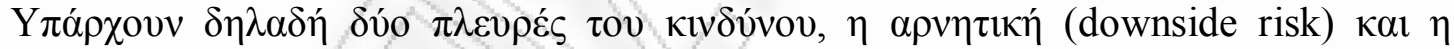

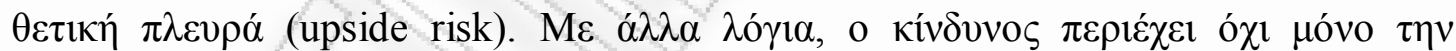

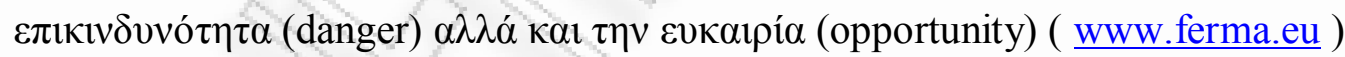

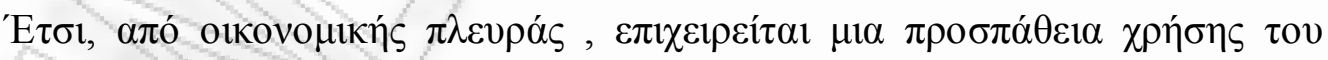

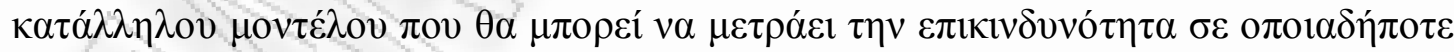

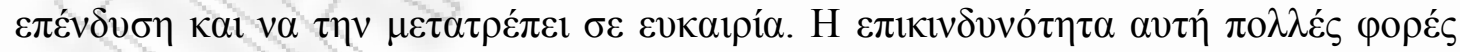

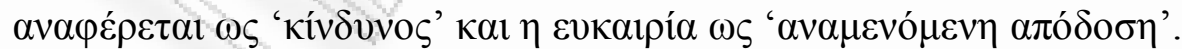




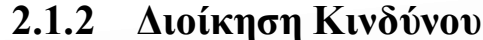

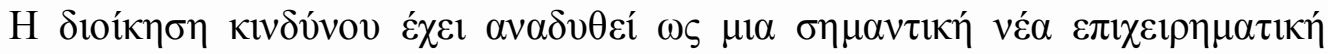

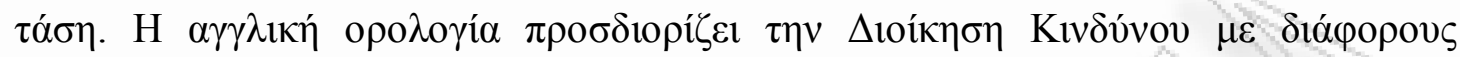
$\tau \rho o ́ \pi о \cup \varsigma$ ó $\omega \varsigma$ Enterprise Risk Management (ERM) каı Integrated Risk Management (IRM).

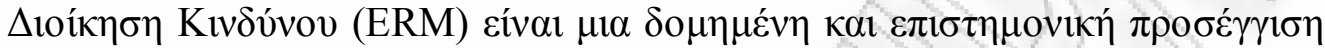

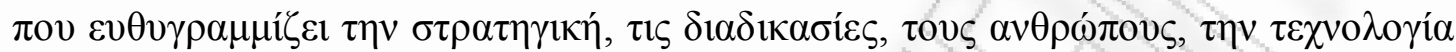

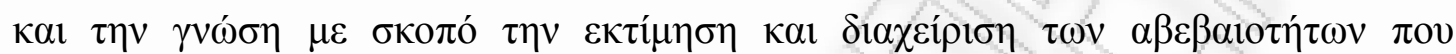

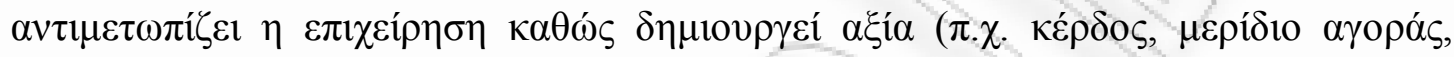

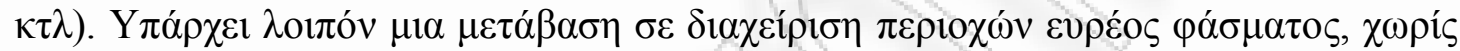

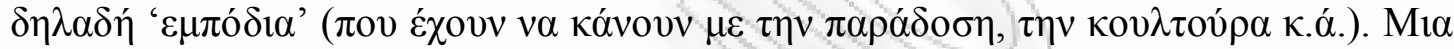

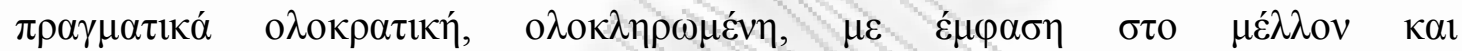

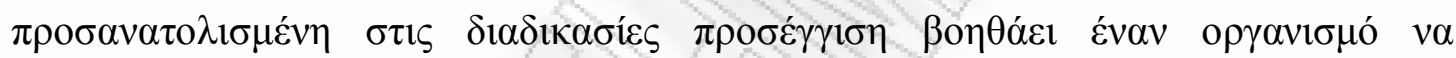

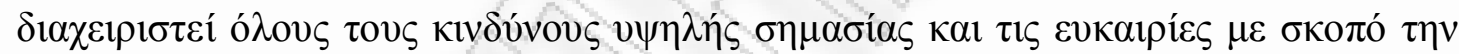

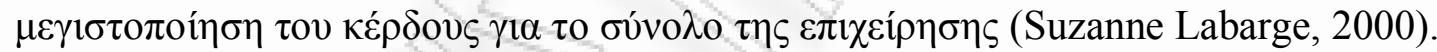

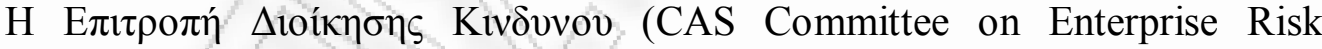

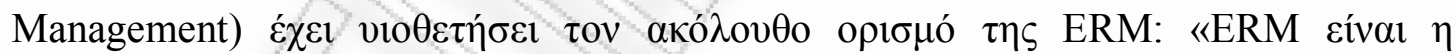

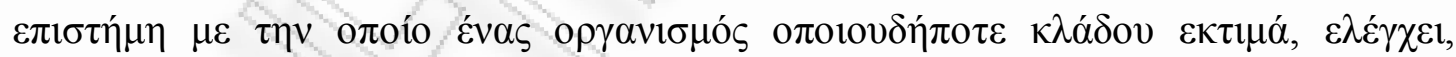

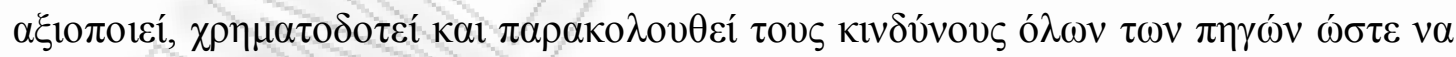
$\alpha v \xi \eta ́ \sigma \varepsilon 1 \tau \alpha \beta \rho \alpha \chi v \pi \rho o ́ \theta \varepsilon \sigma \mu \alpha \kappa \alpha 1 \mu \alpha \kappa \rho о \pi \rho o ́ \theta \varepsilon \sigma \mu \alpha \kappa \varepsilon \dot{\varepsilon} \rho \delta \tau \omega \nu \mu \varepsilon \tau o ́ \chi \omega v$.

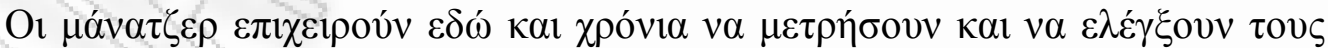

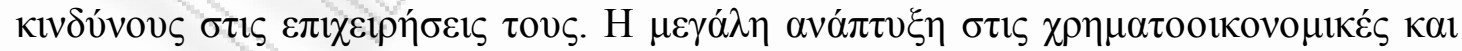

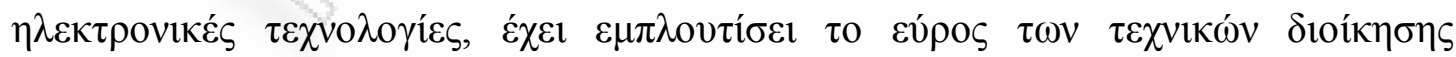

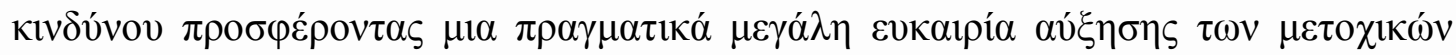

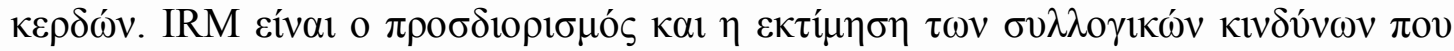




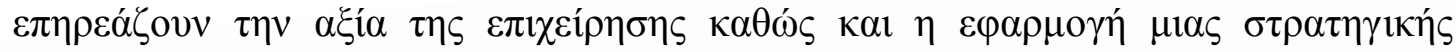

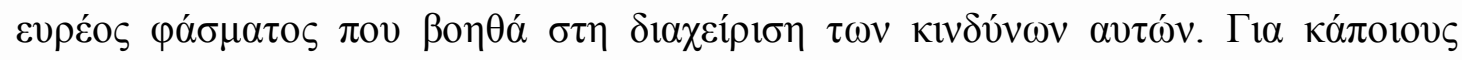

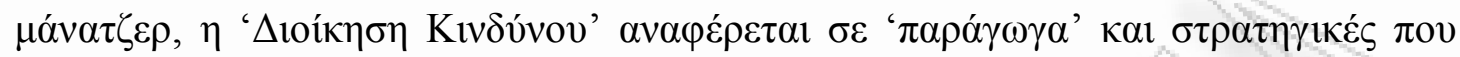

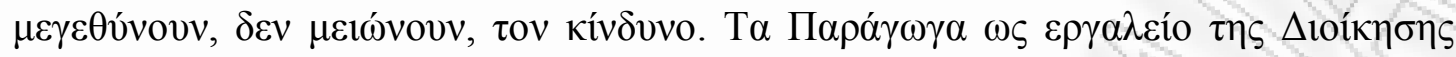

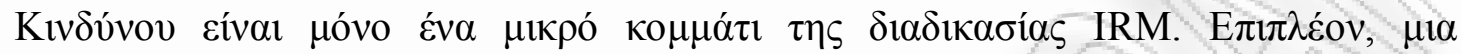

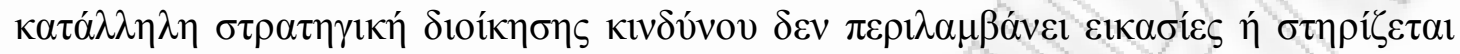

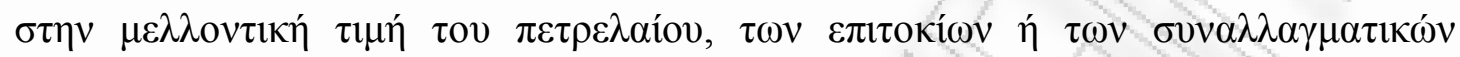

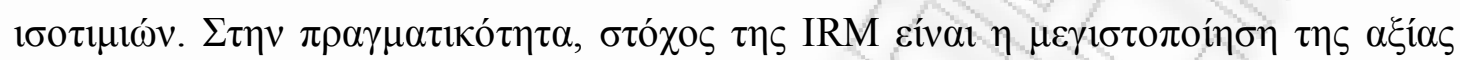

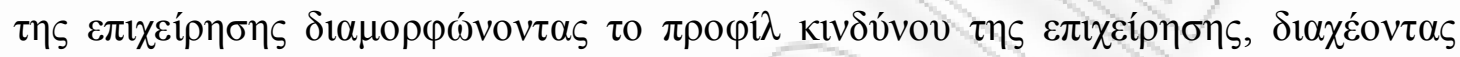

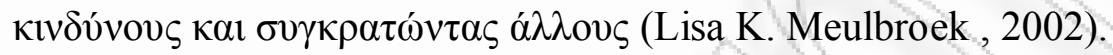

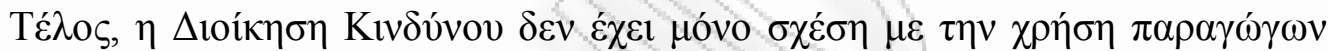

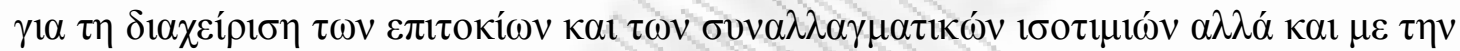

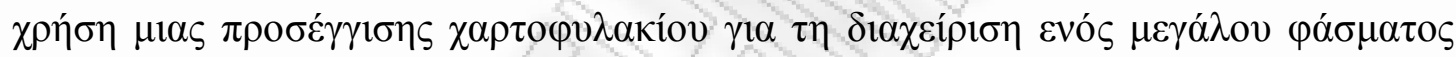

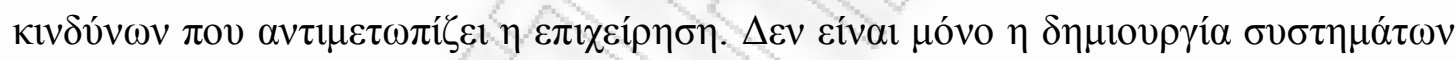

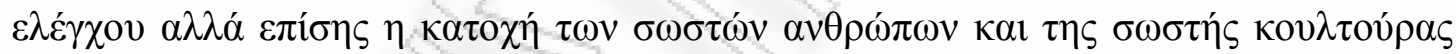

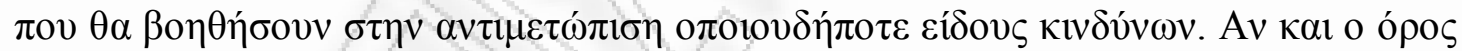

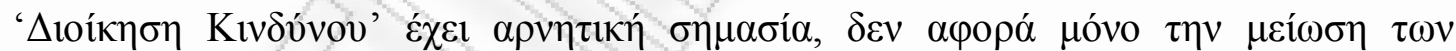

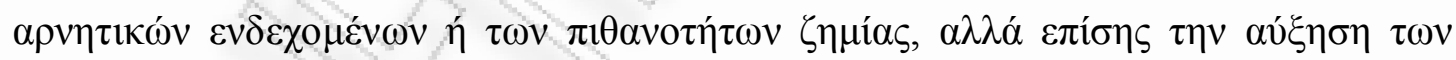

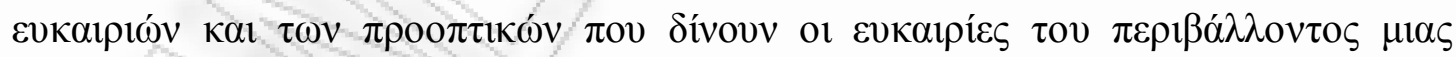

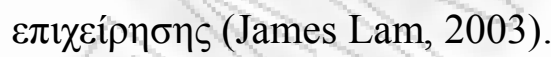

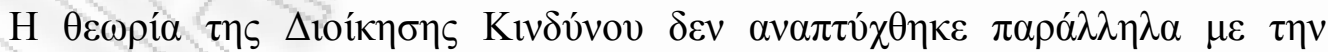

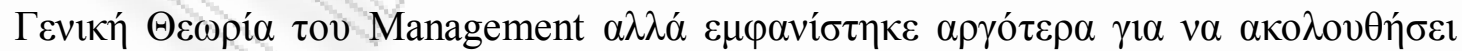

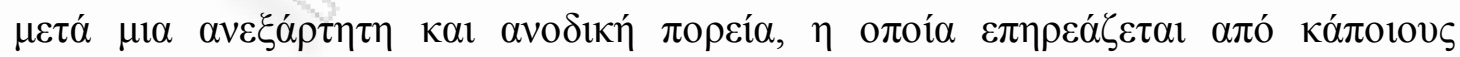

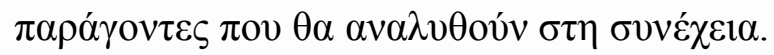




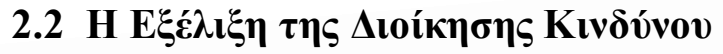

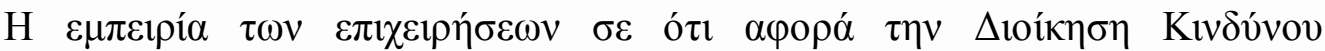

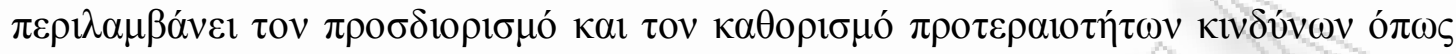

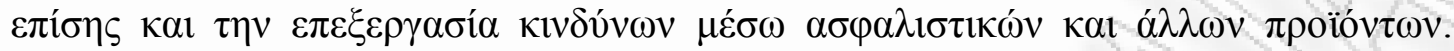

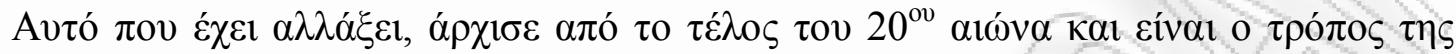

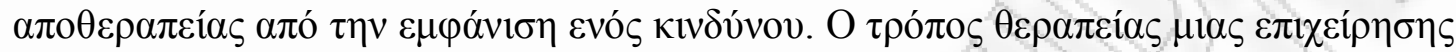

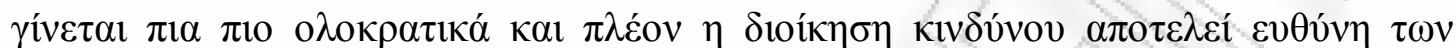

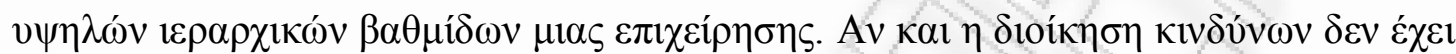

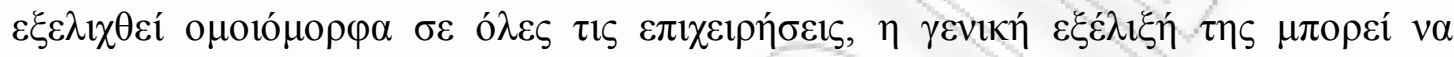

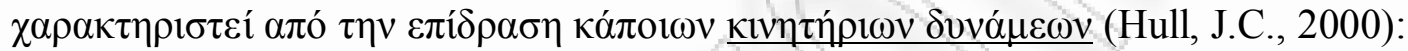

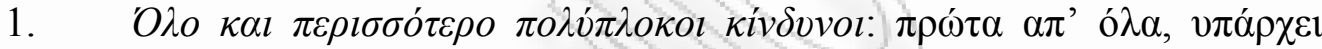

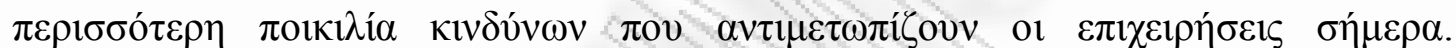

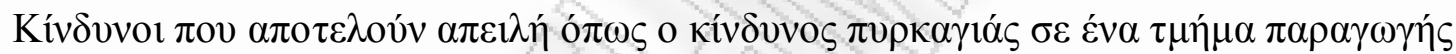

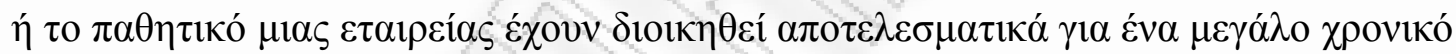

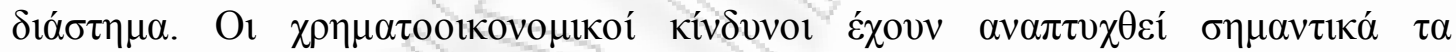

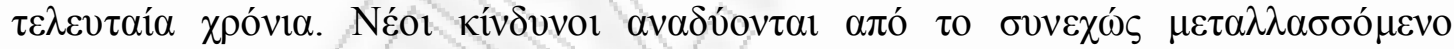

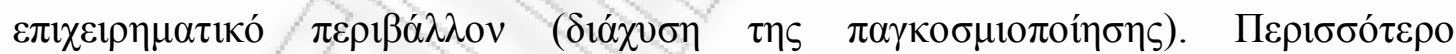

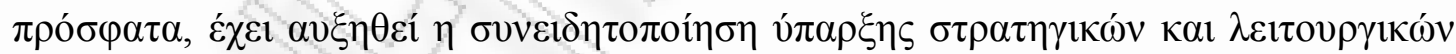

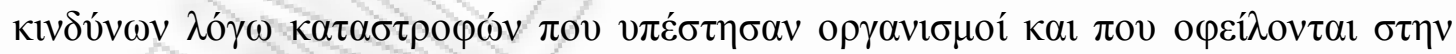

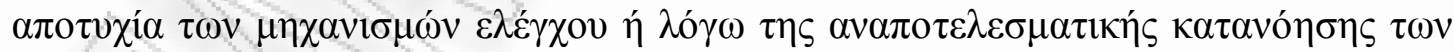

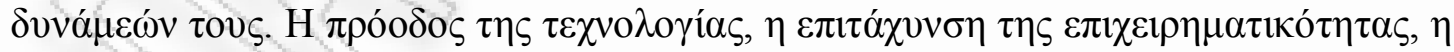

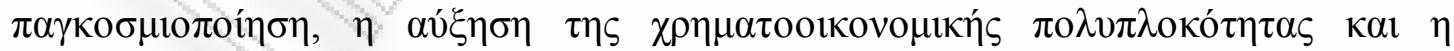

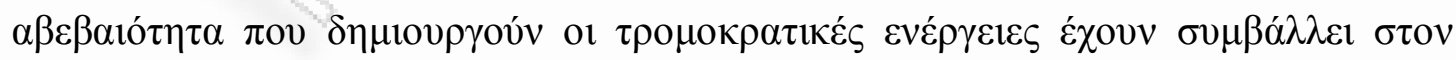

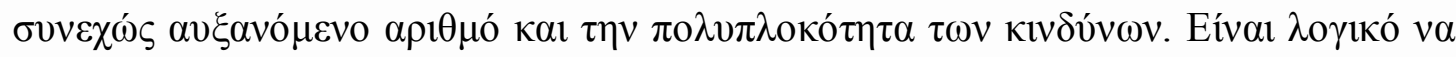

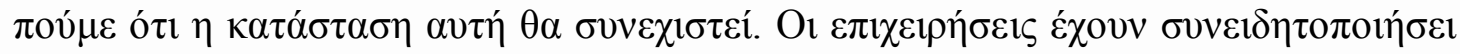




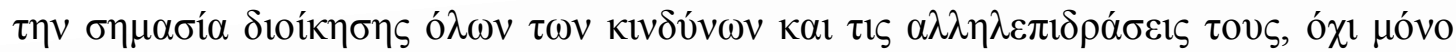

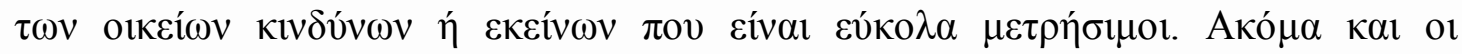

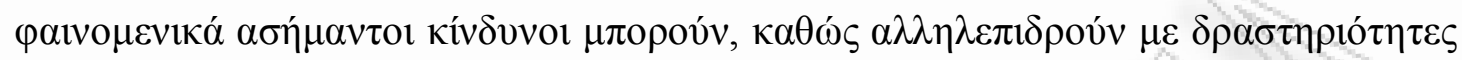

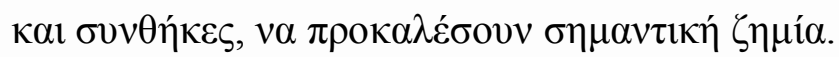

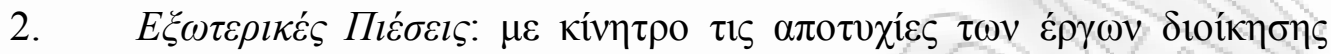

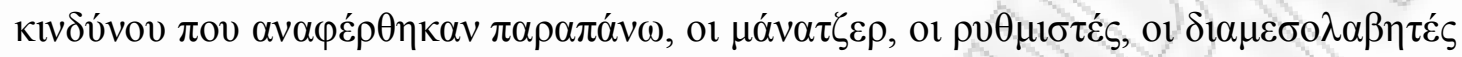

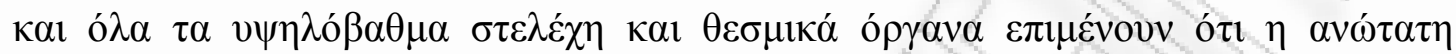

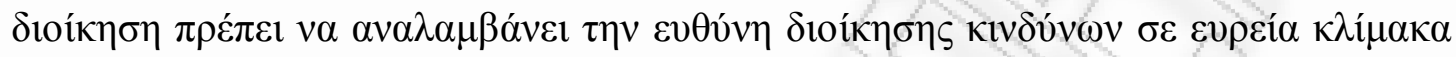

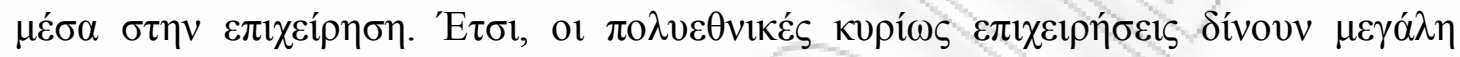

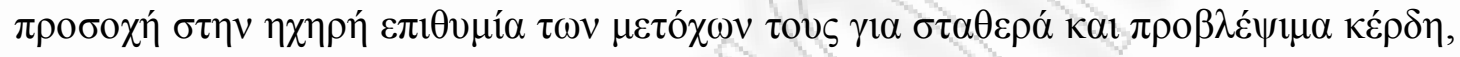

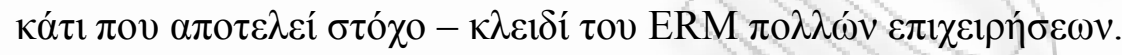

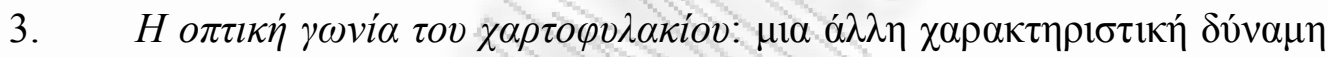

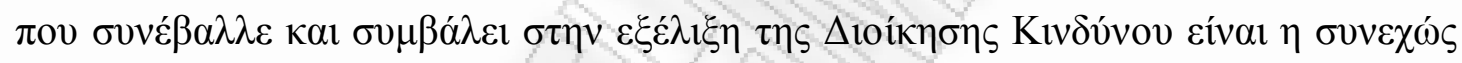

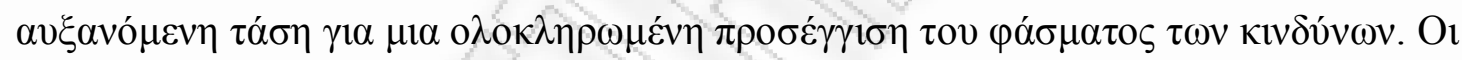

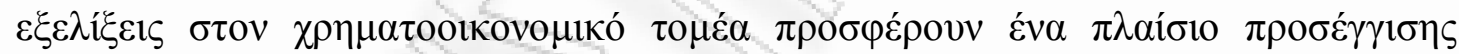

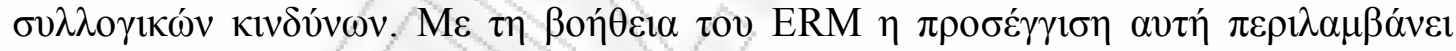

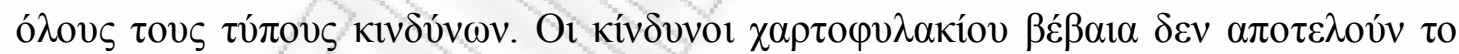

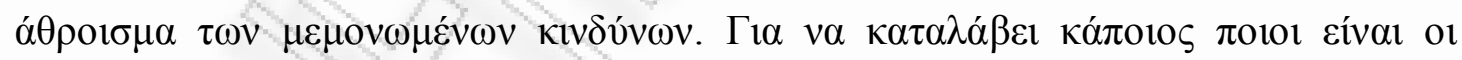

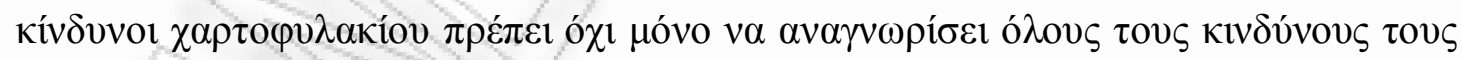

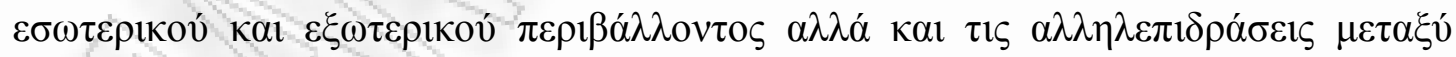

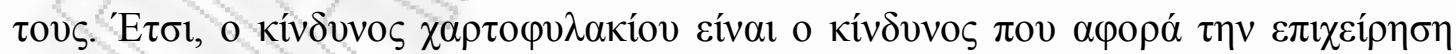

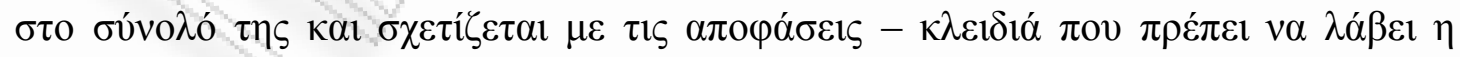

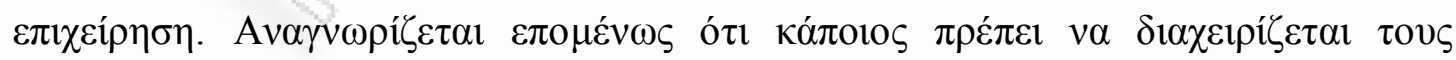

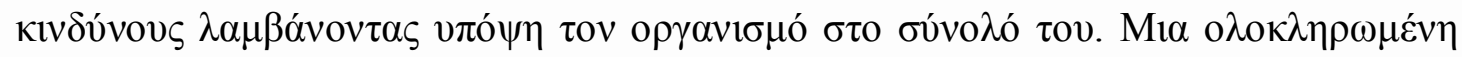




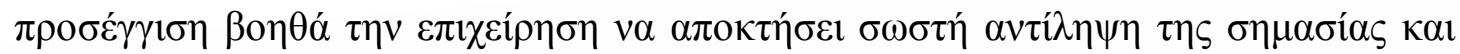

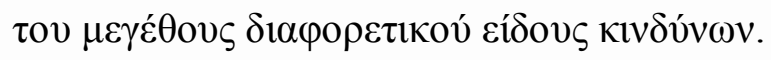

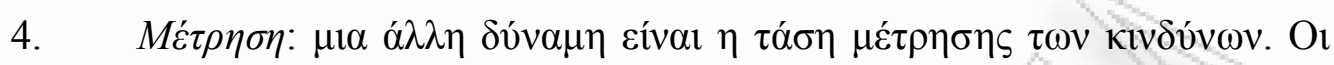

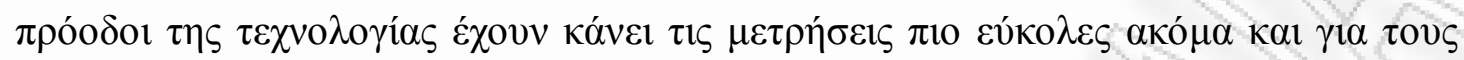

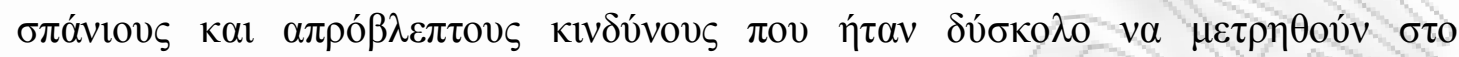

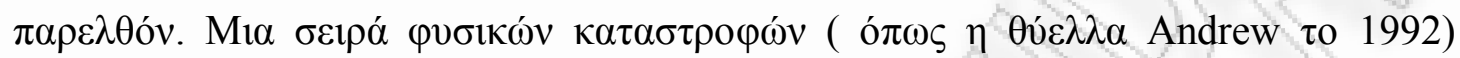

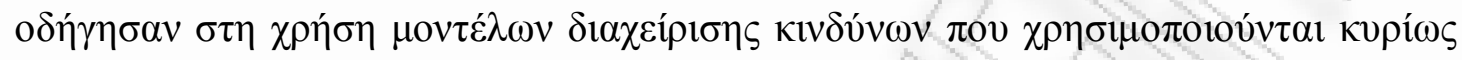

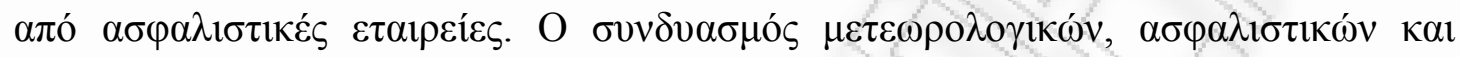

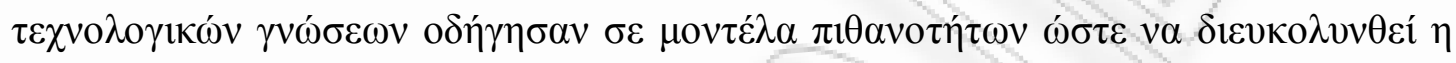

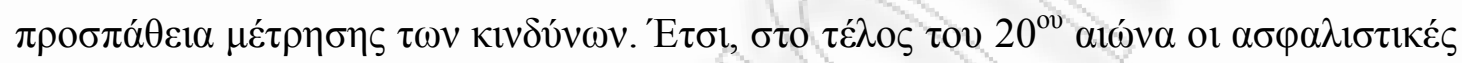

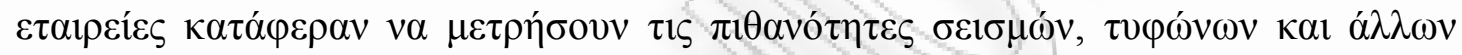

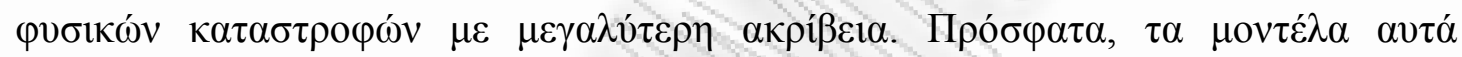

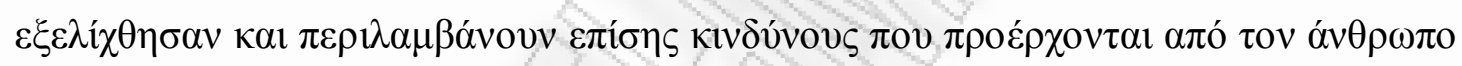

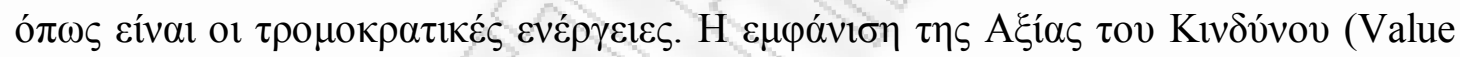

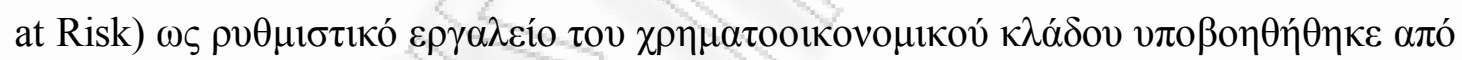

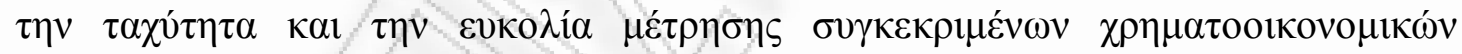

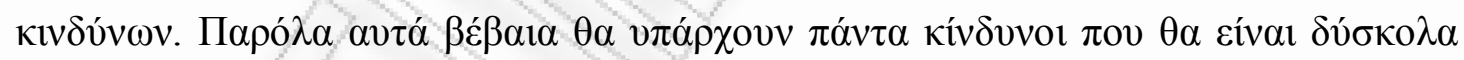

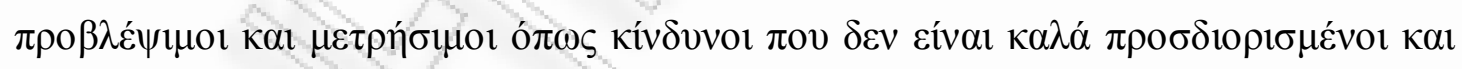

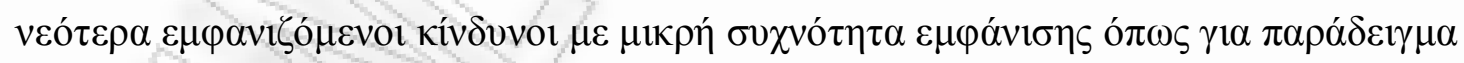

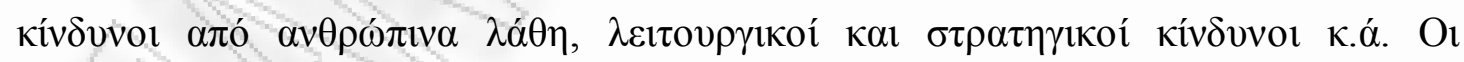

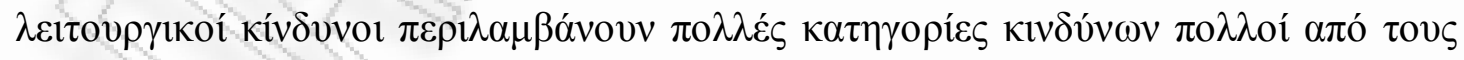

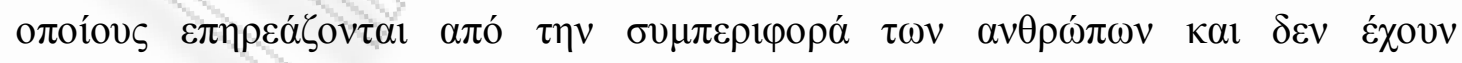

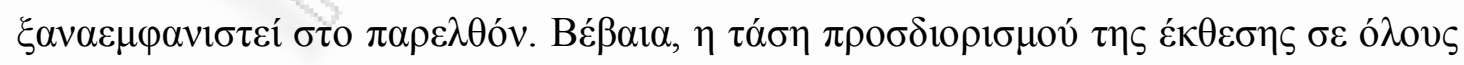

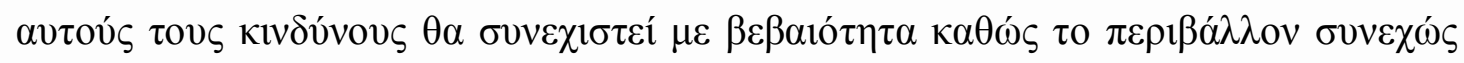

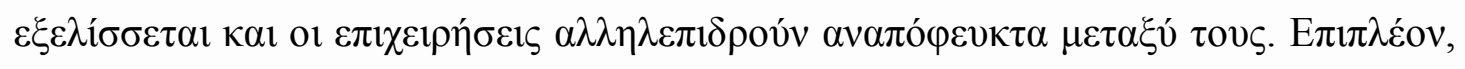




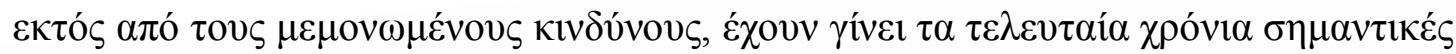

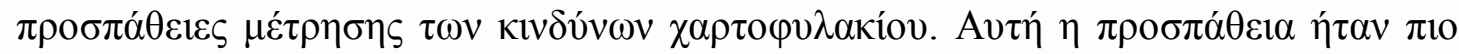

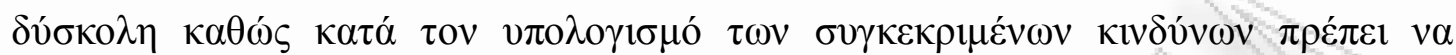

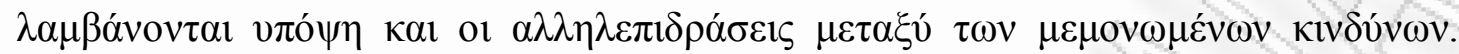

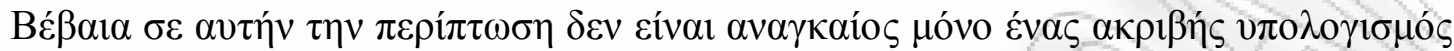

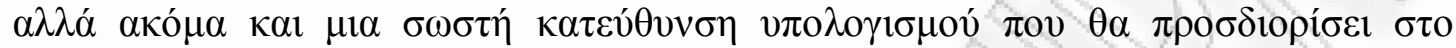

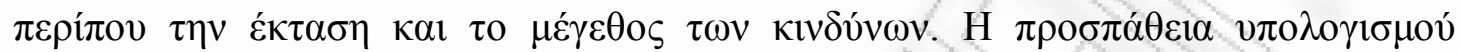

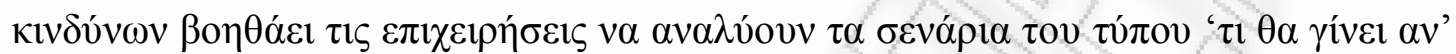
('what if' scenarios).

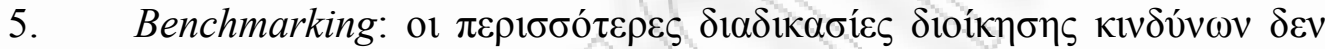

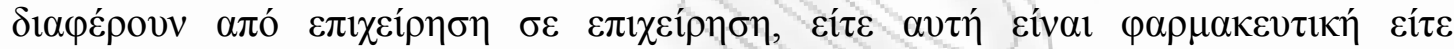

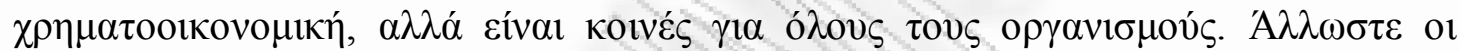

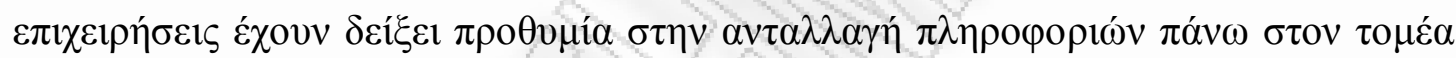

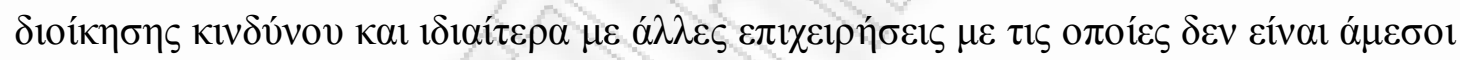

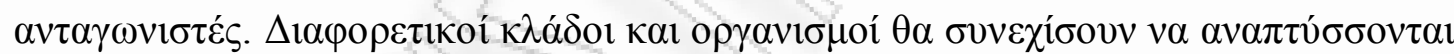

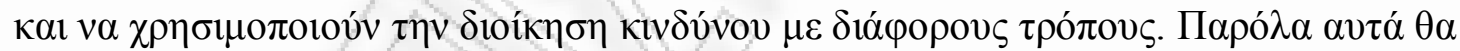

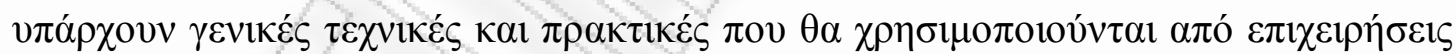

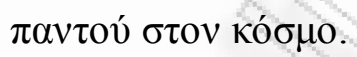

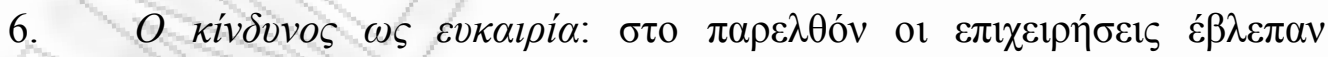

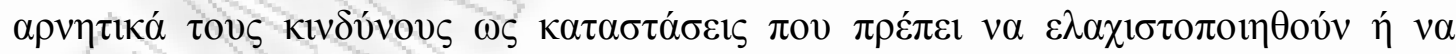

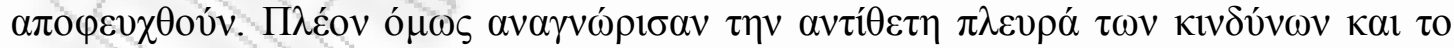

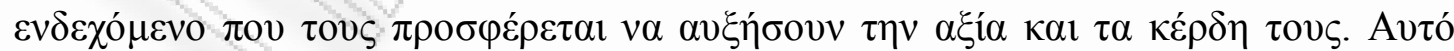

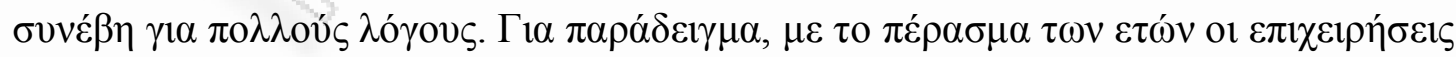

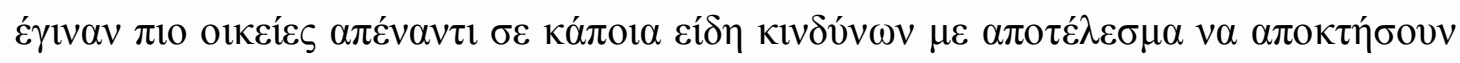

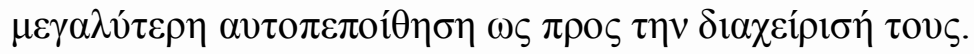




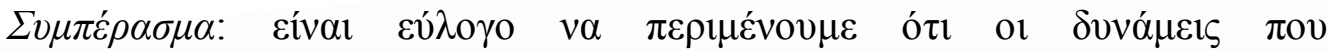

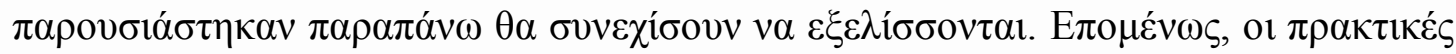

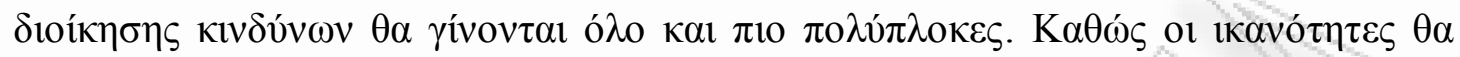

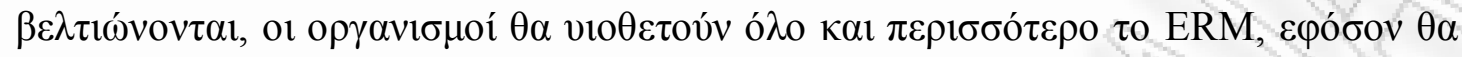

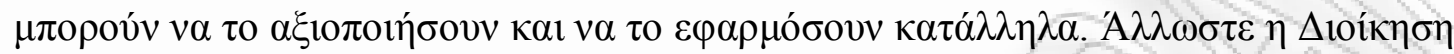

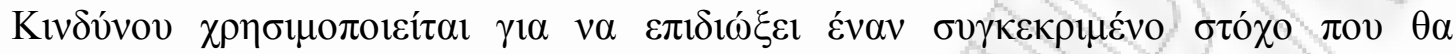

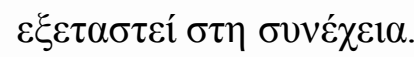

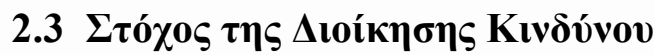

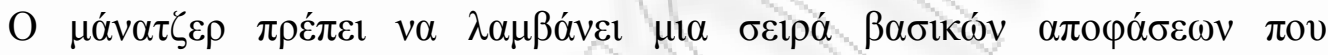

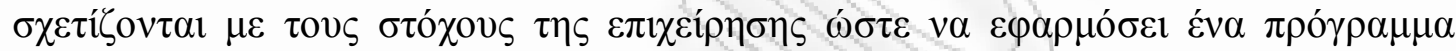

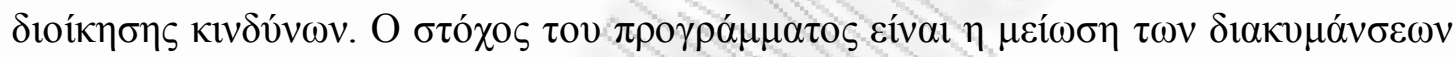

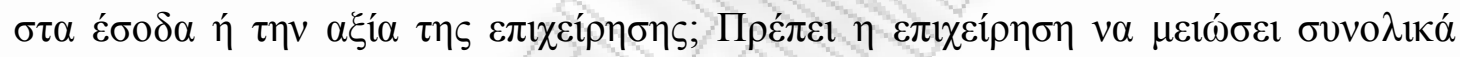

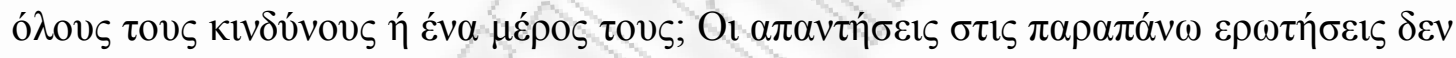

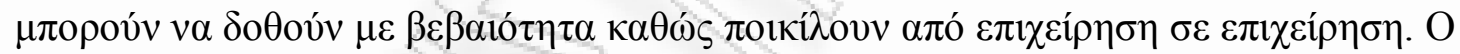

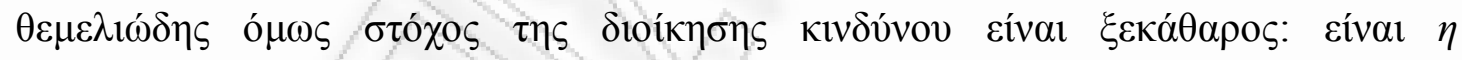

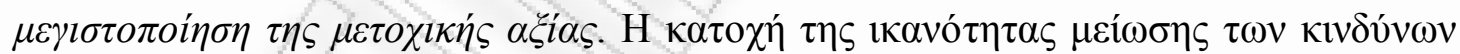

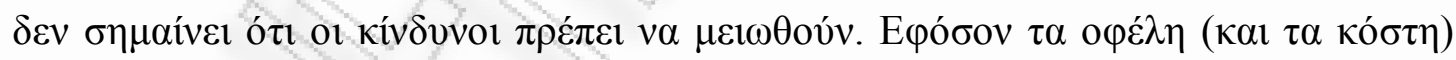

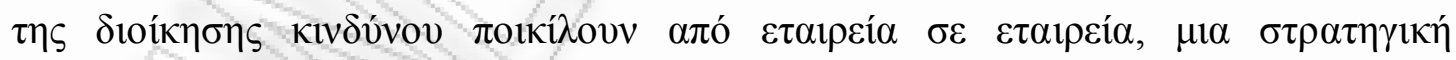

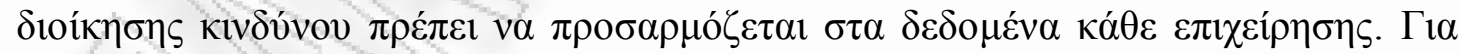

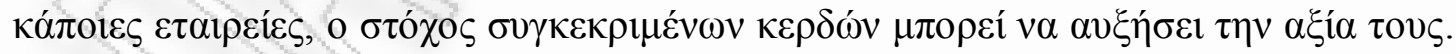

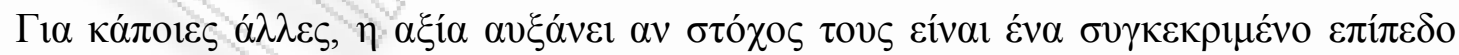

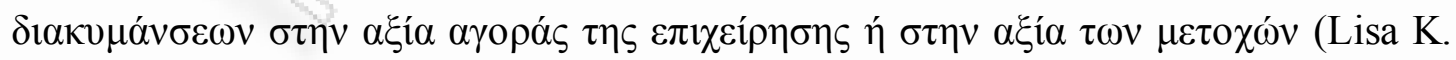
Meulbroek, 2002). 


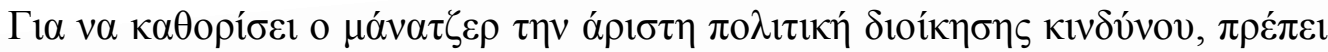

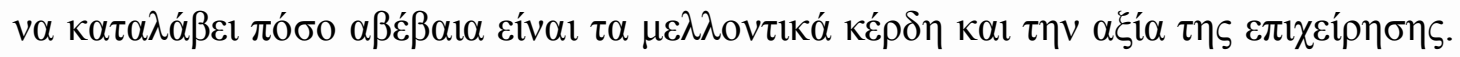

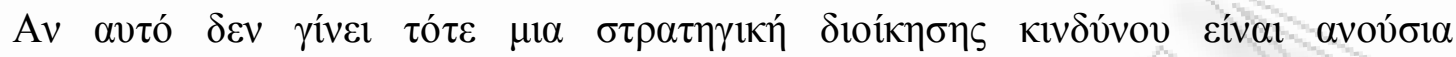
(www.tillinghast.com).

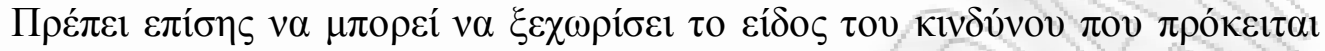

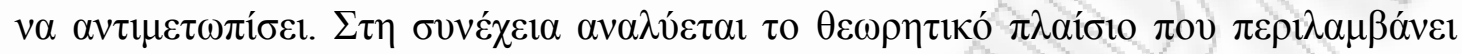

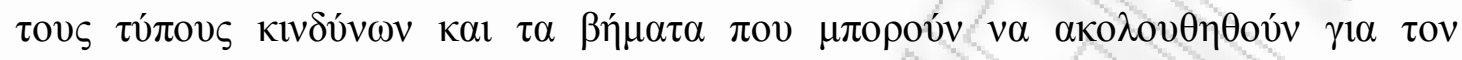

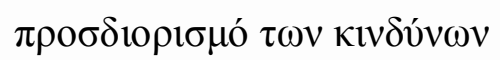

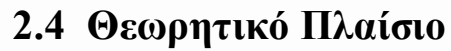

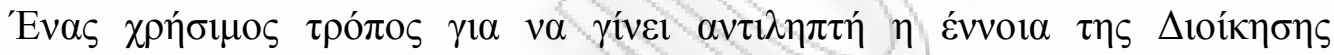

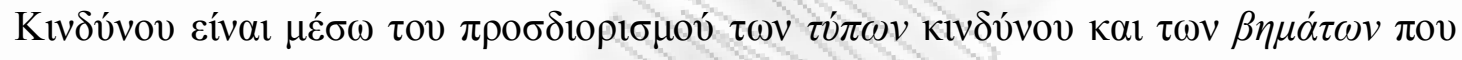

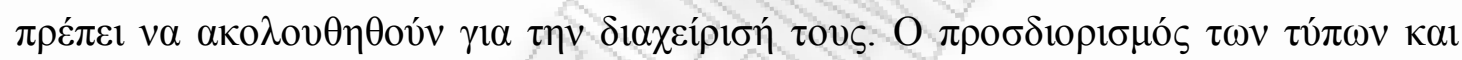

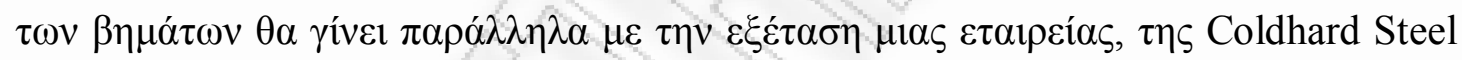

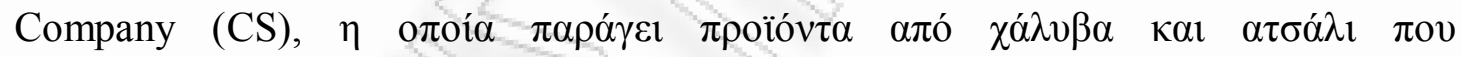

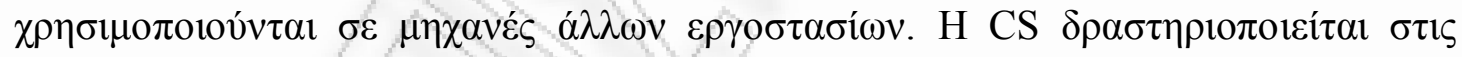

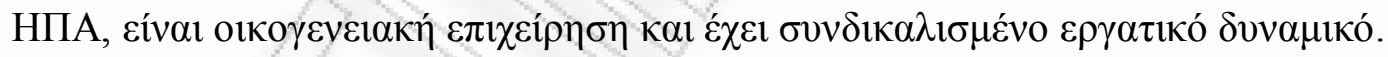

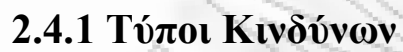

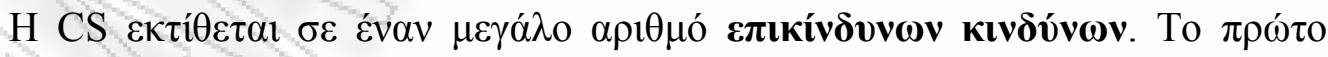

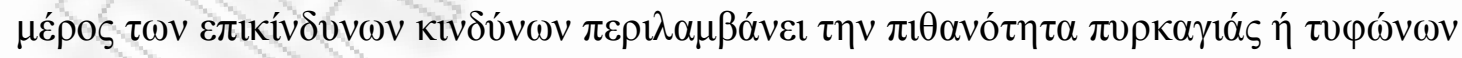

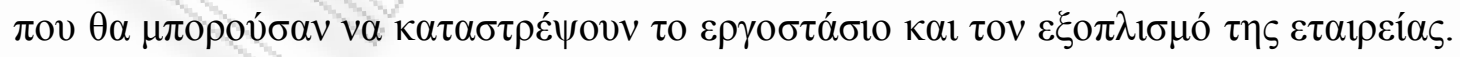

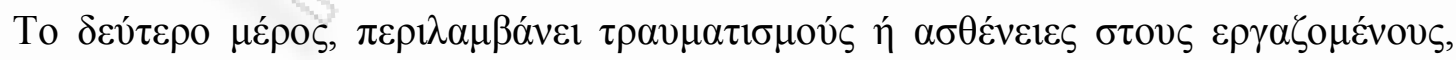

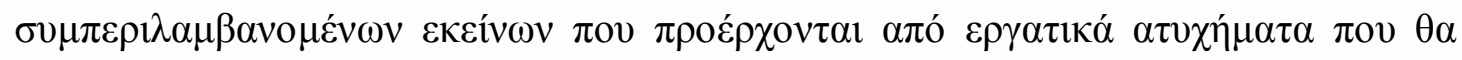

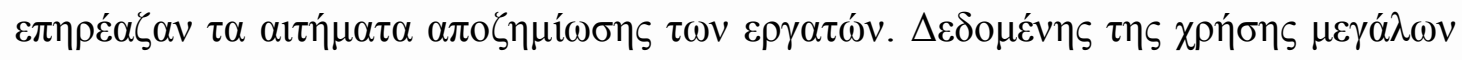




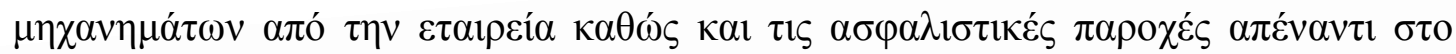

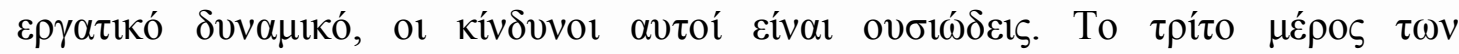

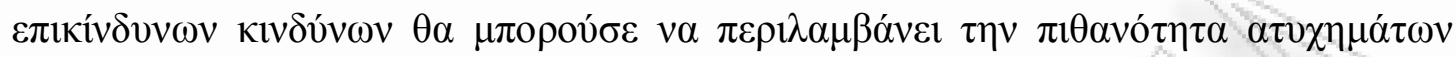

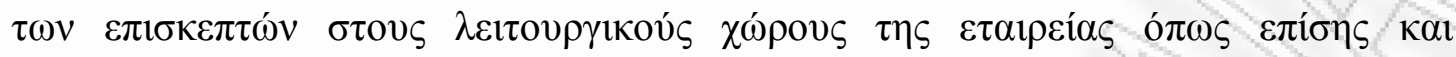

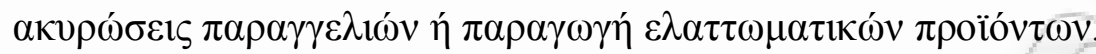

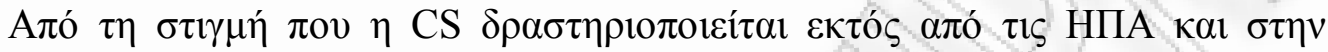

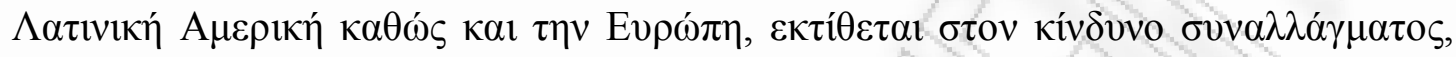

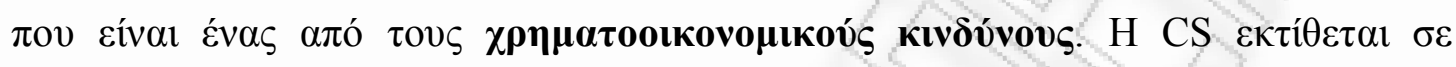

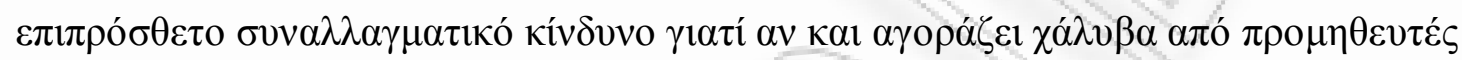

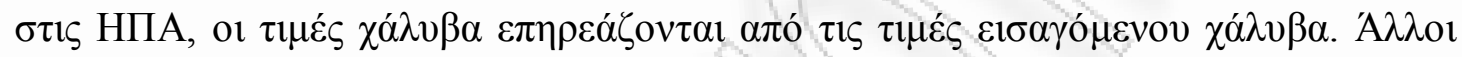

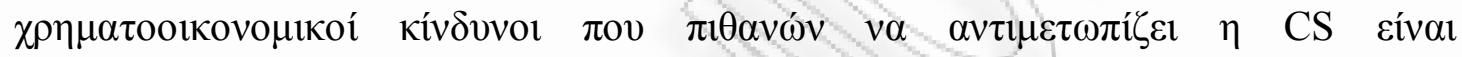

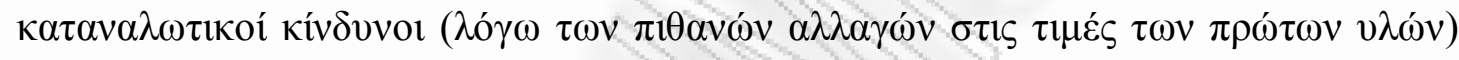

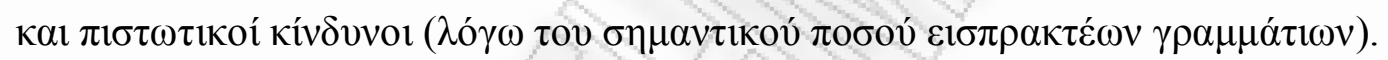

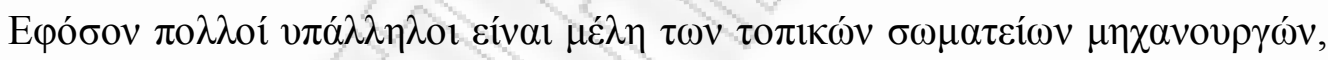

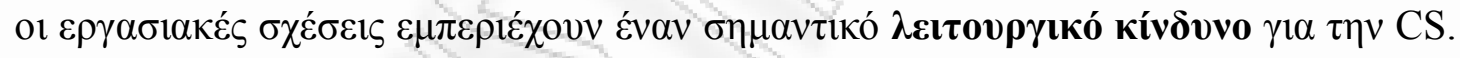

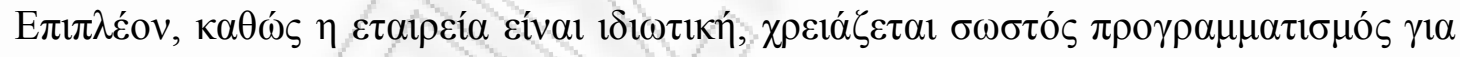

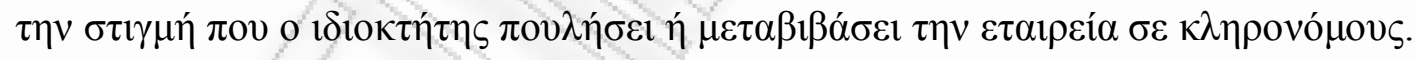

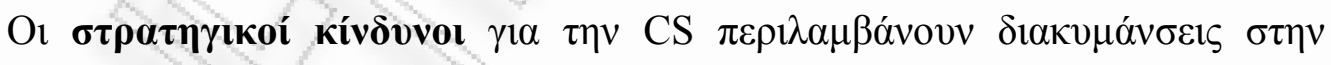

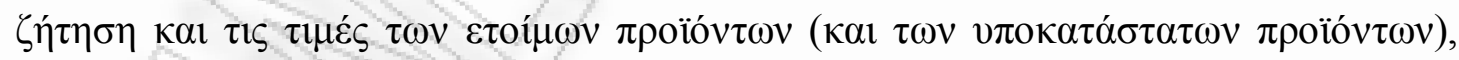

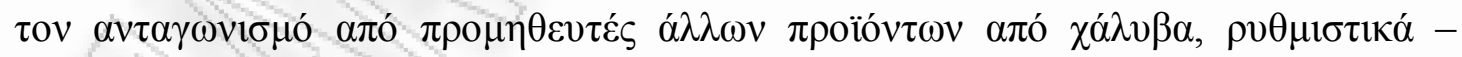

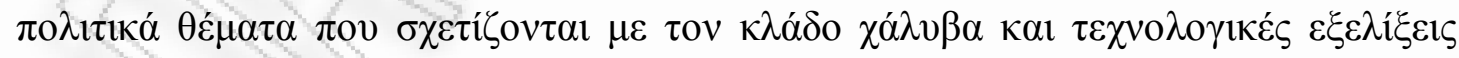

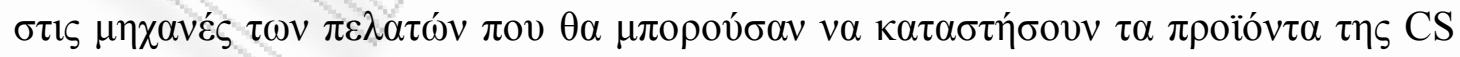
$\xi \varepsilon \pi \varepsilon \rho \alpha \sigma \mu \varepsilon \dot{v} \alpha$. 


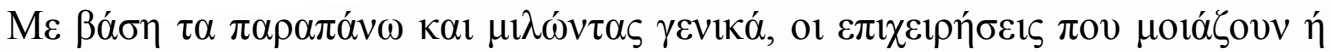

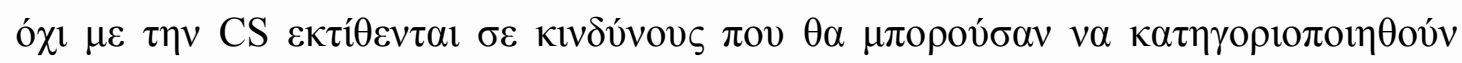

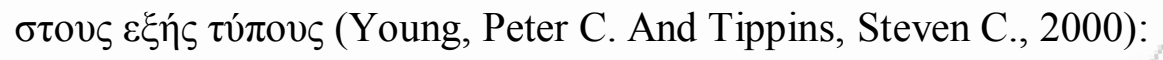

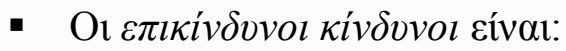

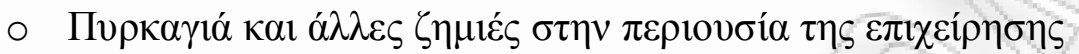

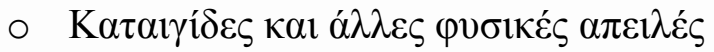

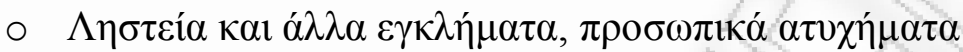

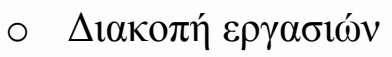

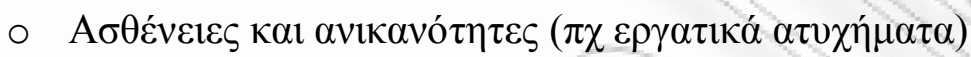

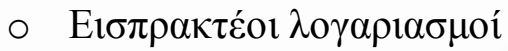

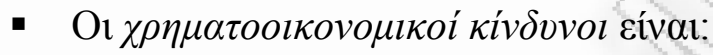

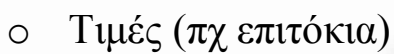

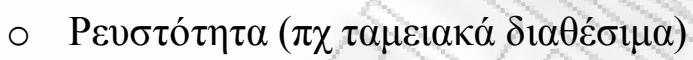

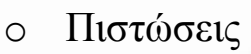

- $\Pi \lambda \eta \theta \omega \rho ı \mu$ ós

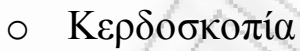

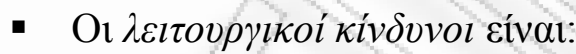

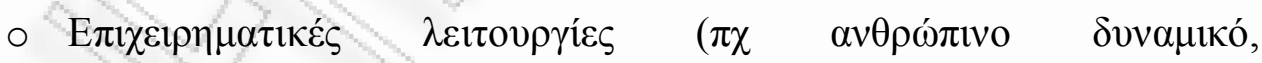

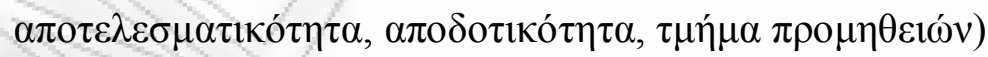

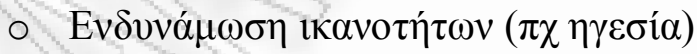

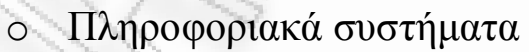

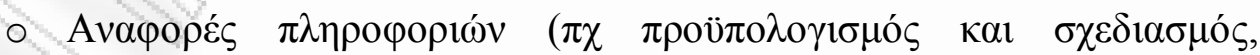

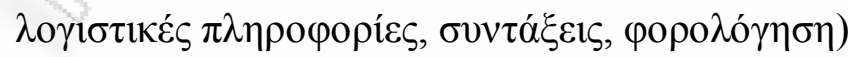

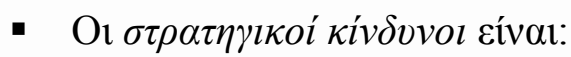

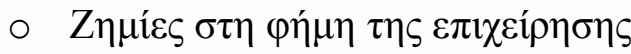




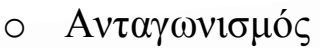
○ $\quad \mathrm{E} \pi \theta v \mu i ́ \varepsilon \varsigma \pi \varepsilon \lambda \alpha \tau \omega ́ v$

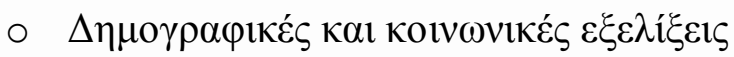

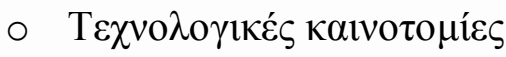
○ $\Delta 1 \alpha \theta \varepsilon \sigma \mu \operatorname{có}_{\tau \eta \tau} \alpha \kappa \varepsilon \varphi \alpha \lambda \alpha i ́ \omega v$

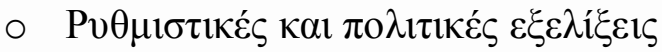

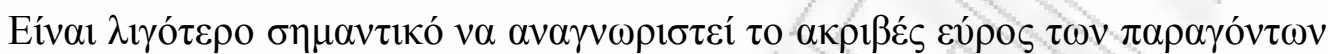

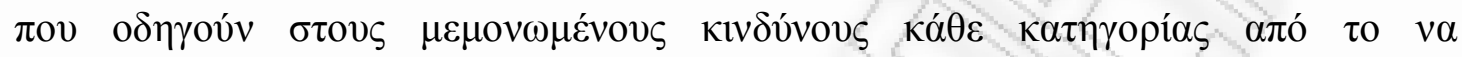

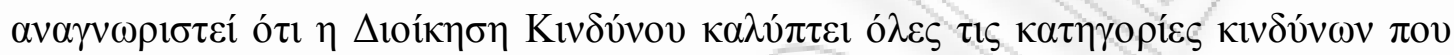

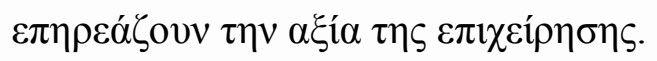

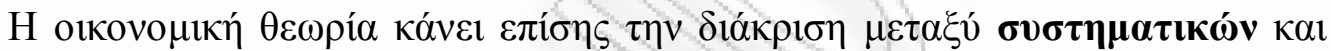

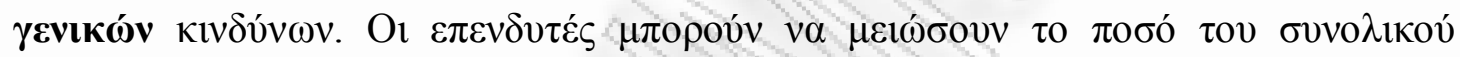

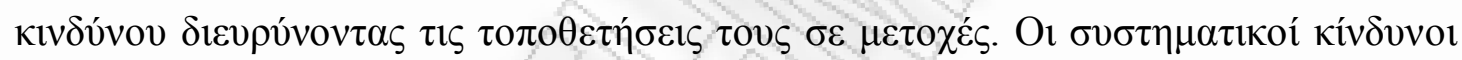

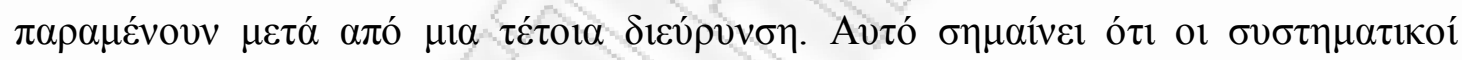

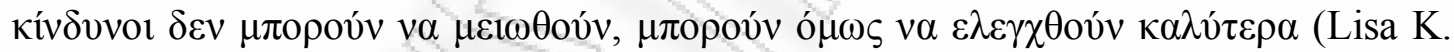
Meulbroek, 2002).

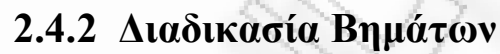

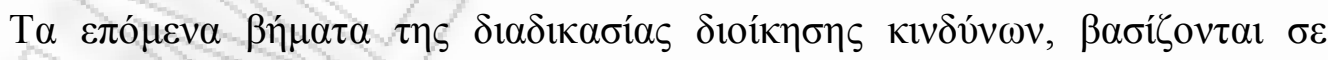

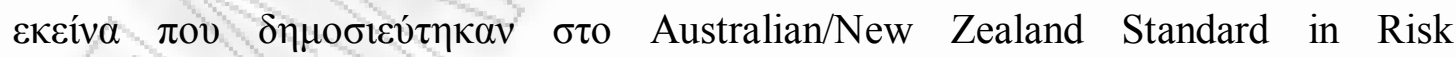

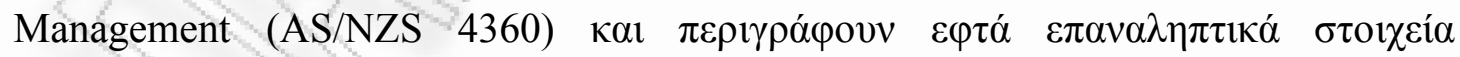
(www.standards.org): 
$\Sigma \chi \eta ́ \mu \alpha$ 1: $\Delta 1 \alpha \delta \iota \kappa \alpha \sigma i ́ \alpha \beta \eta \mu \alpha ́ \tau \omega \nu$

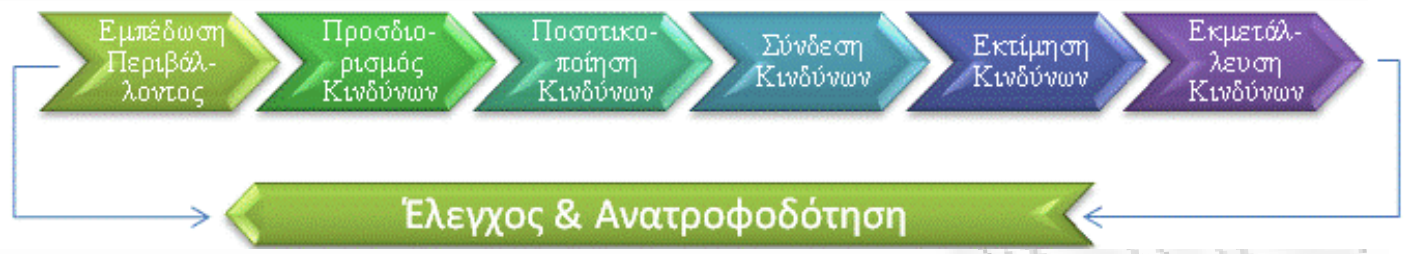

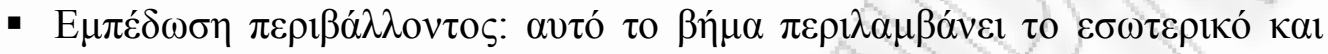

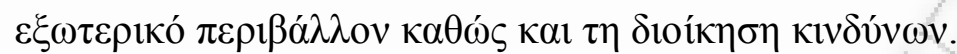

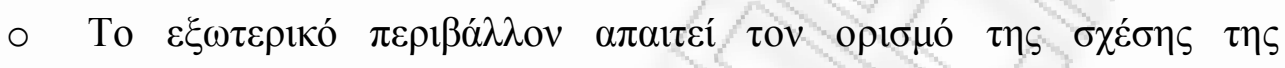

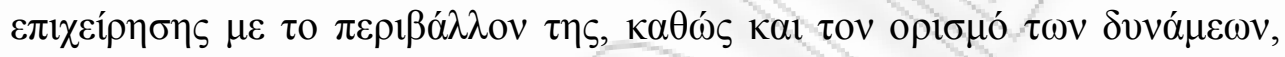

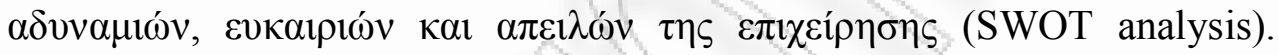

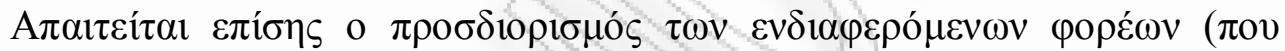

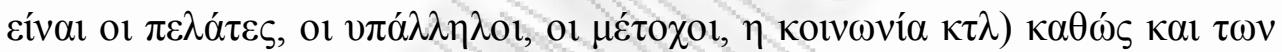

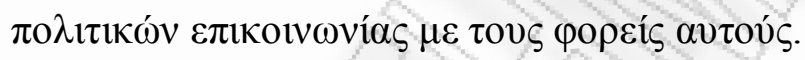

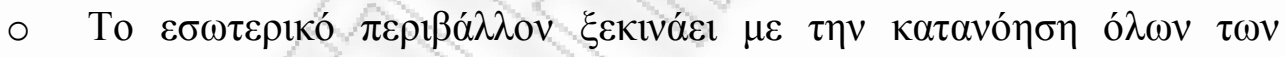

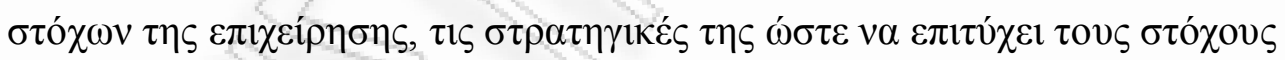

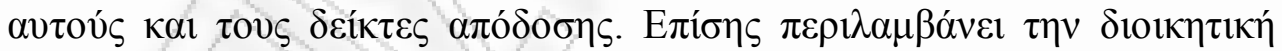

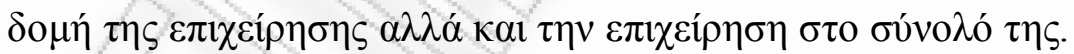

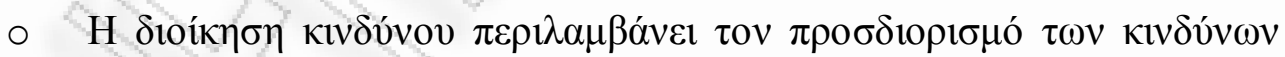

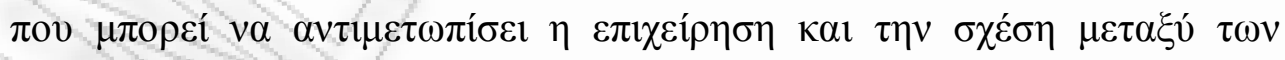

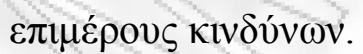

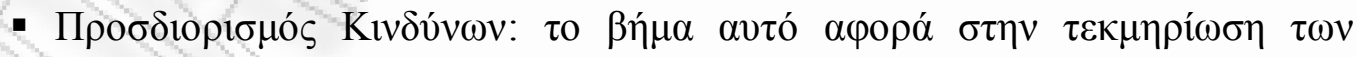

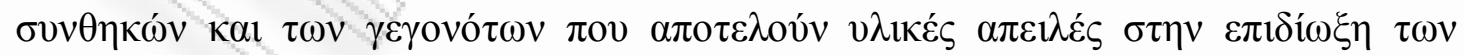

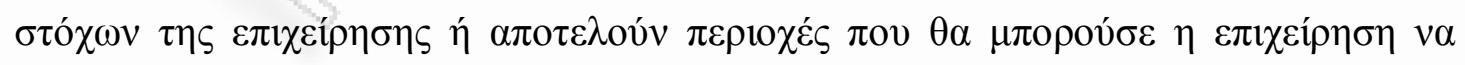

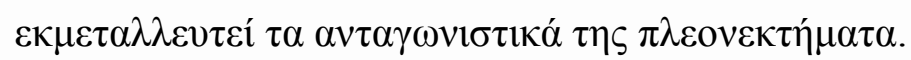




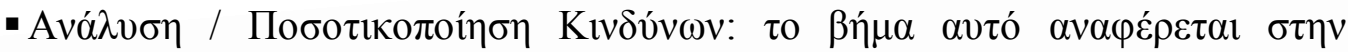

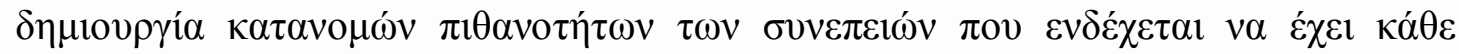

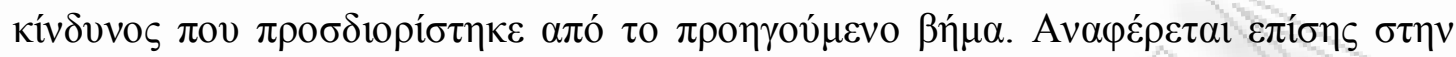

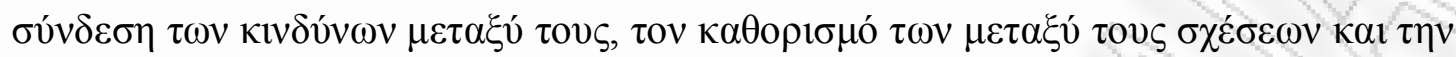

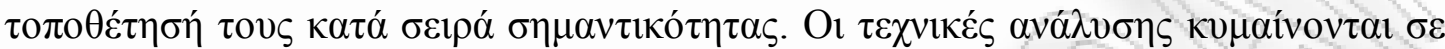

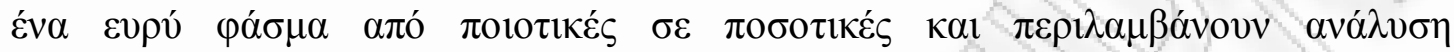

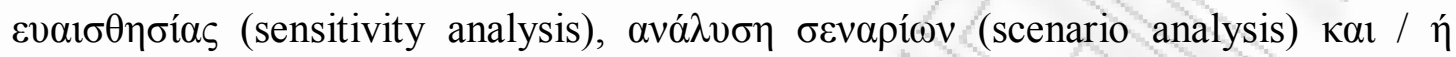

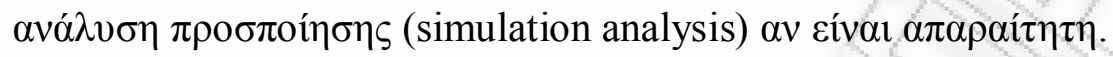

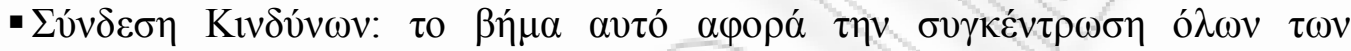

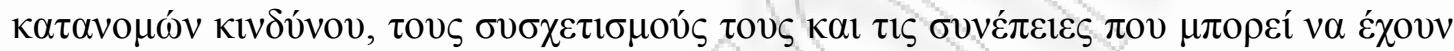

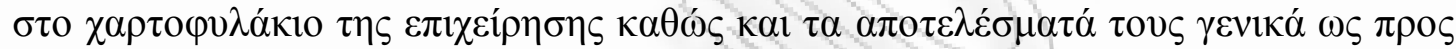

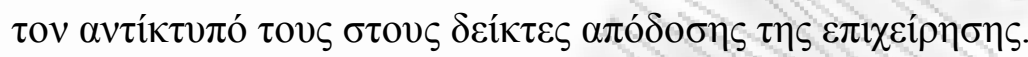

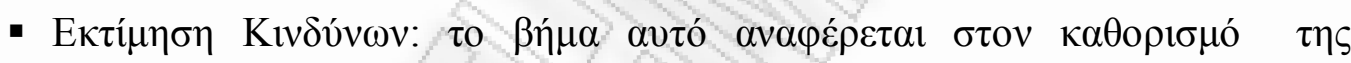

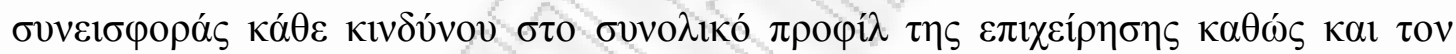

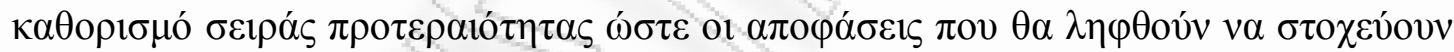

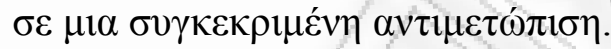

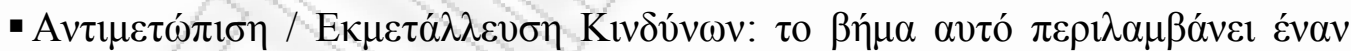

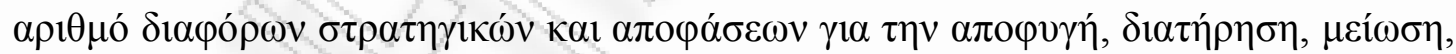

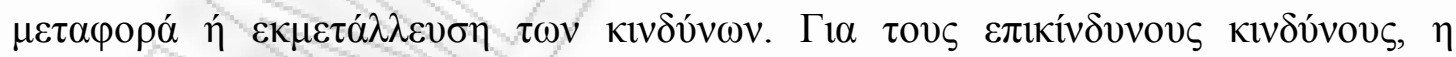

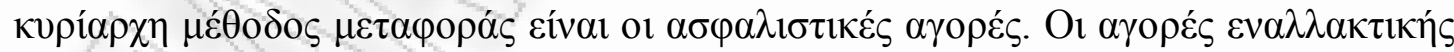

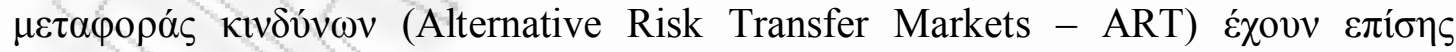

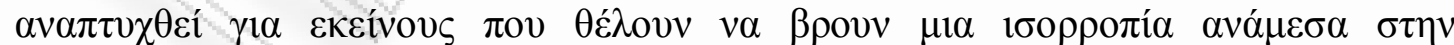

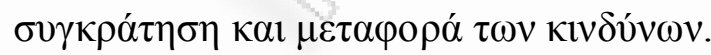

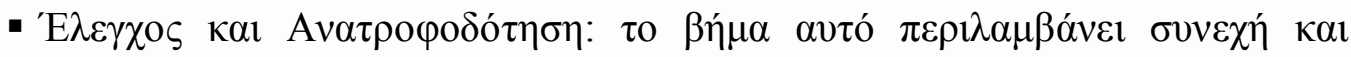

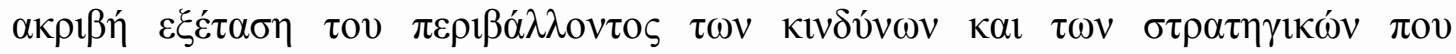




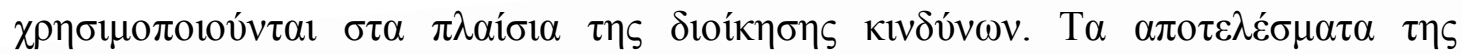

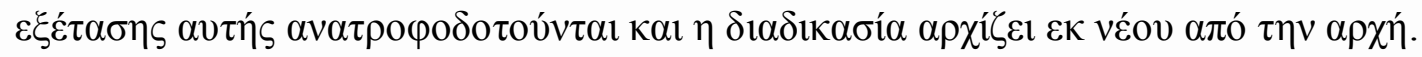

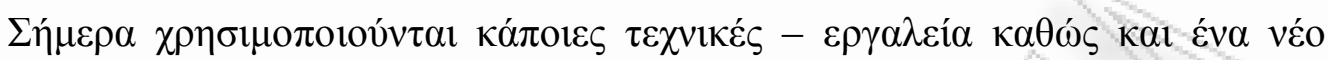

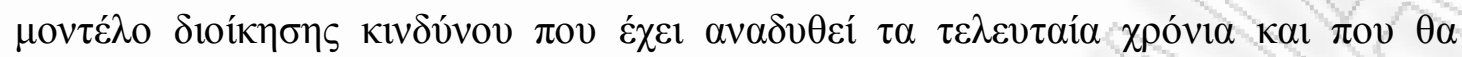

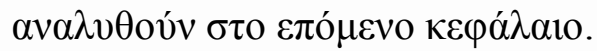




\section{КЕФАААIO 3}

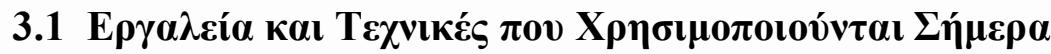

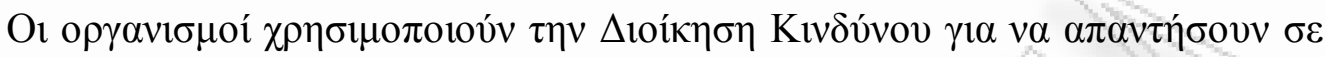

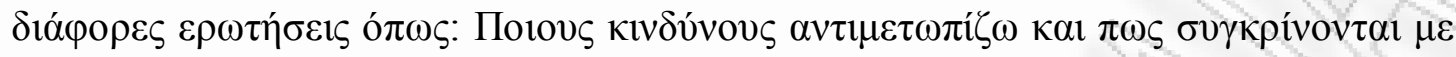

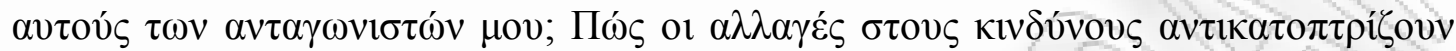

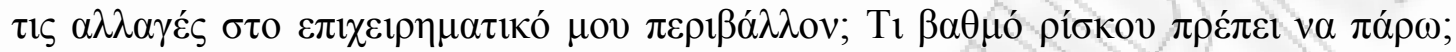

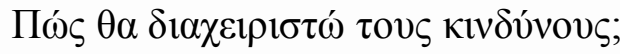

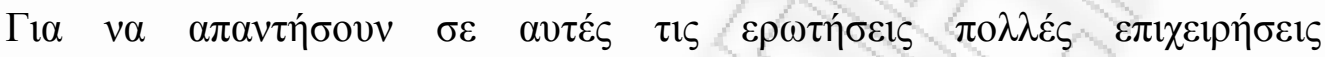

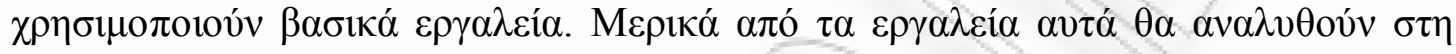

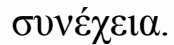

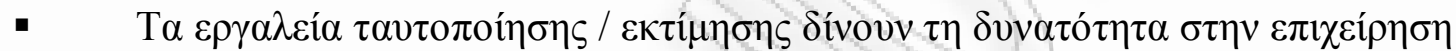

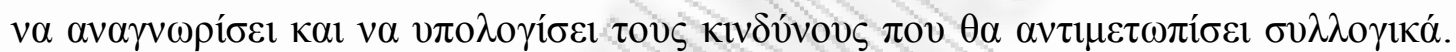

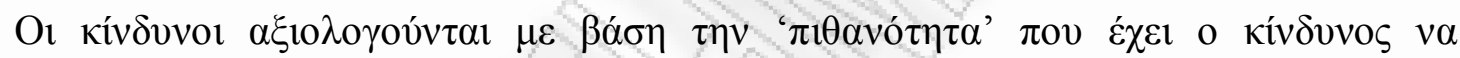

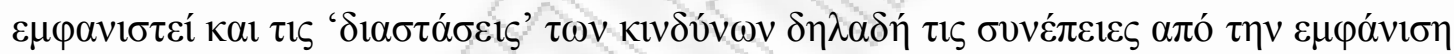

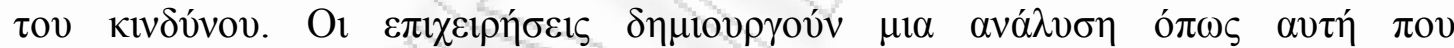

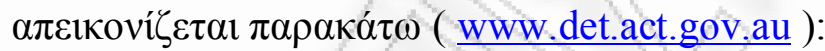




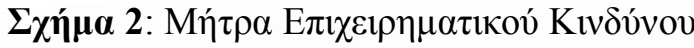

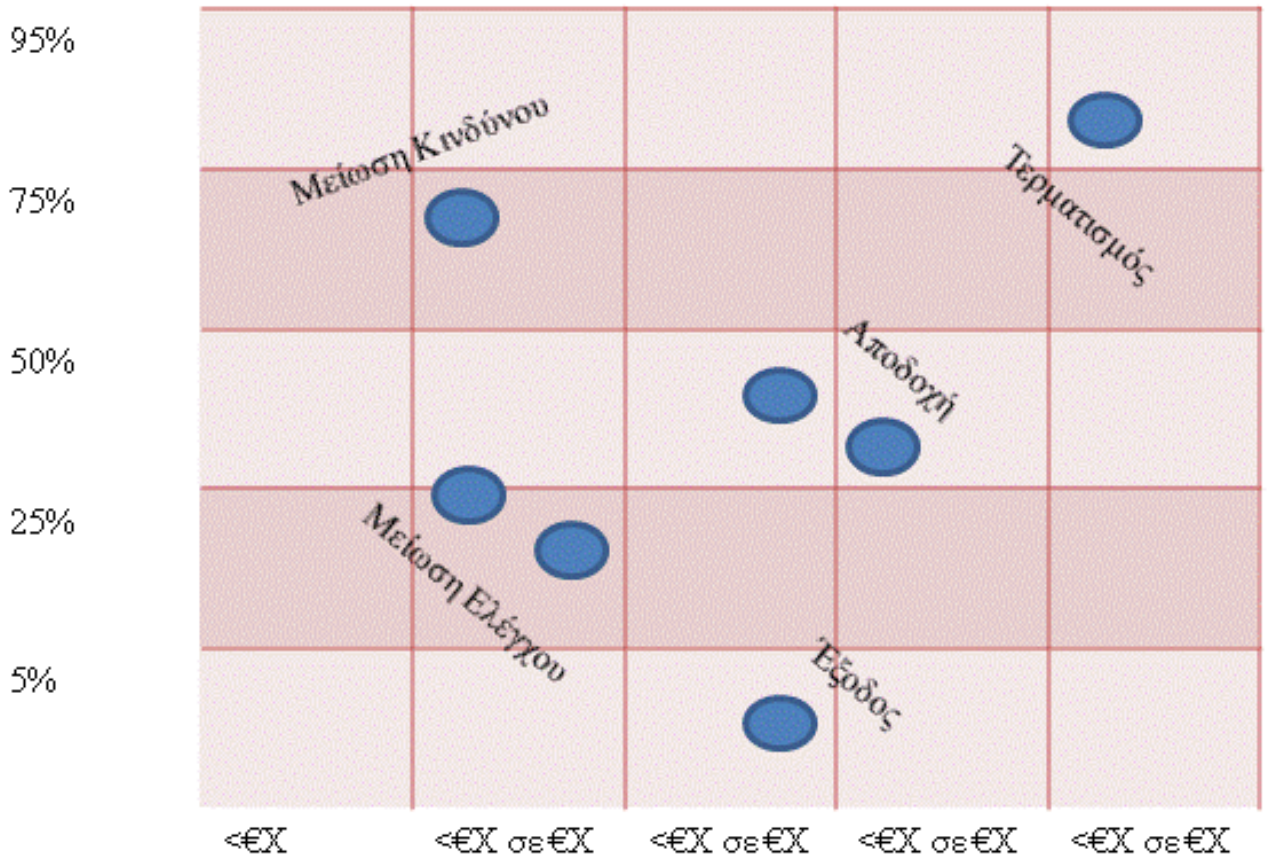

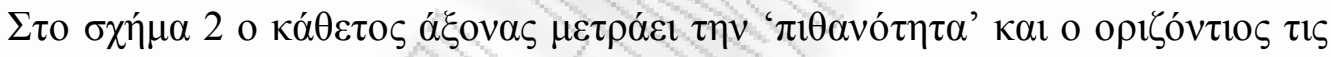

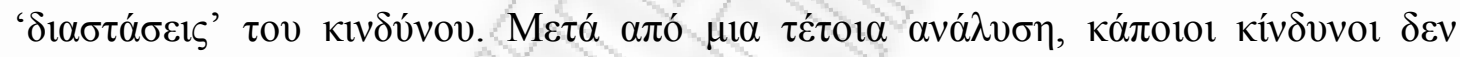

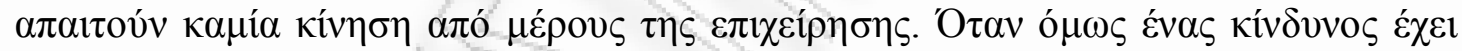

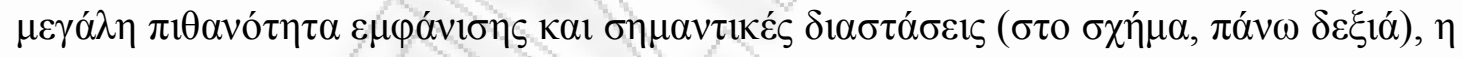

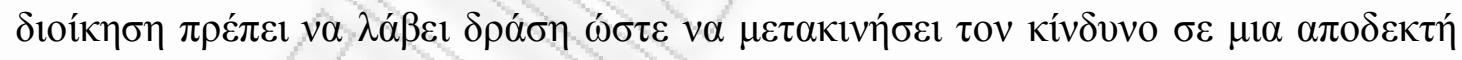

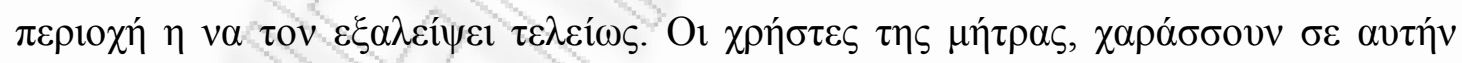

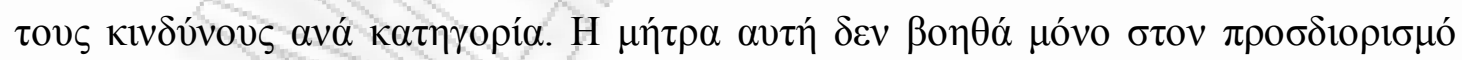

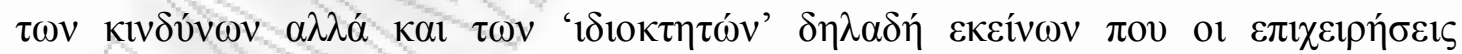

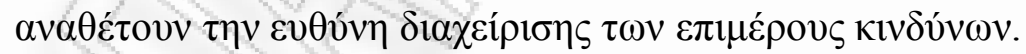

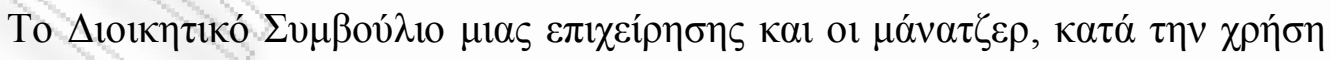

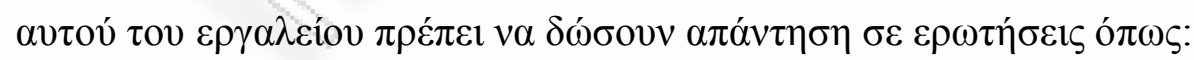

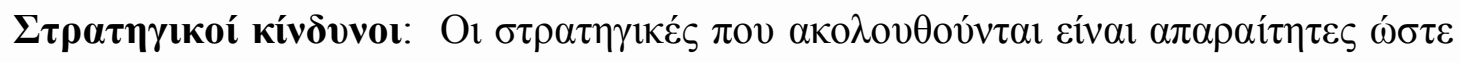

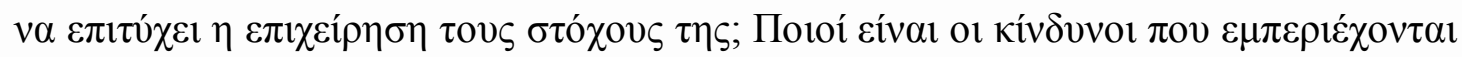




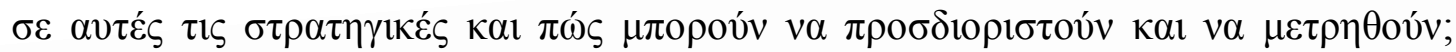

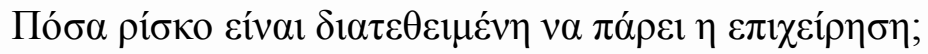

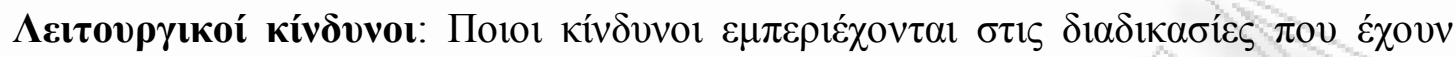

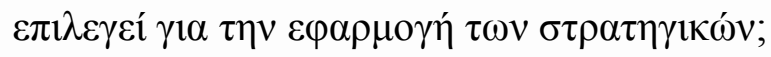

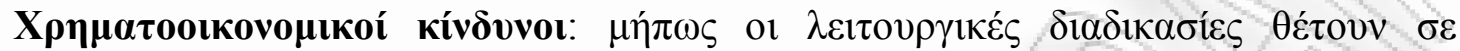

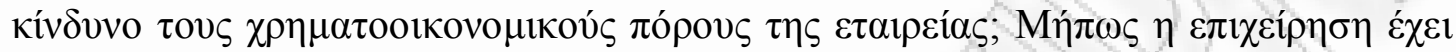

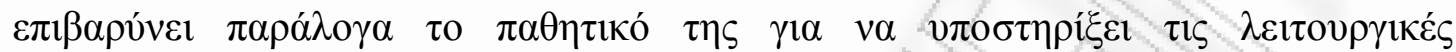

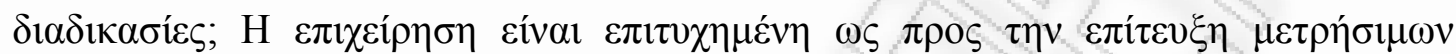
$\varepsilon \pi \imath \chi \varepsilon \imath \rho \eta \mu \alpha \tau \imath \kappa \omega ́ v ~ \sigma \tau o ́ \chi \omega v ;$

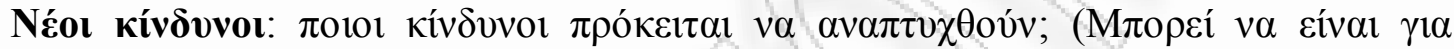

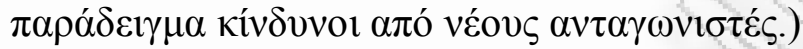

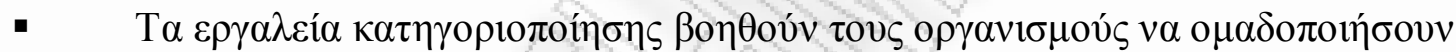

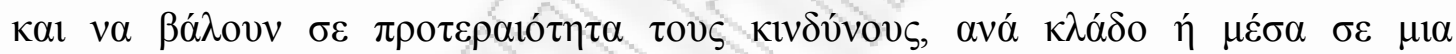

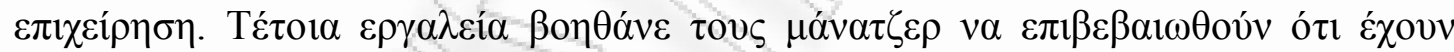

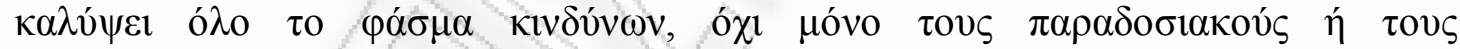

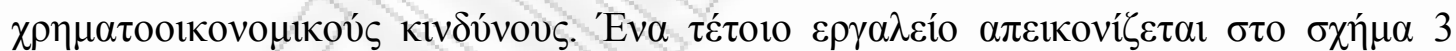
( www.findarticles.com): 


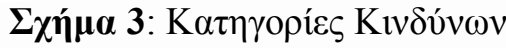

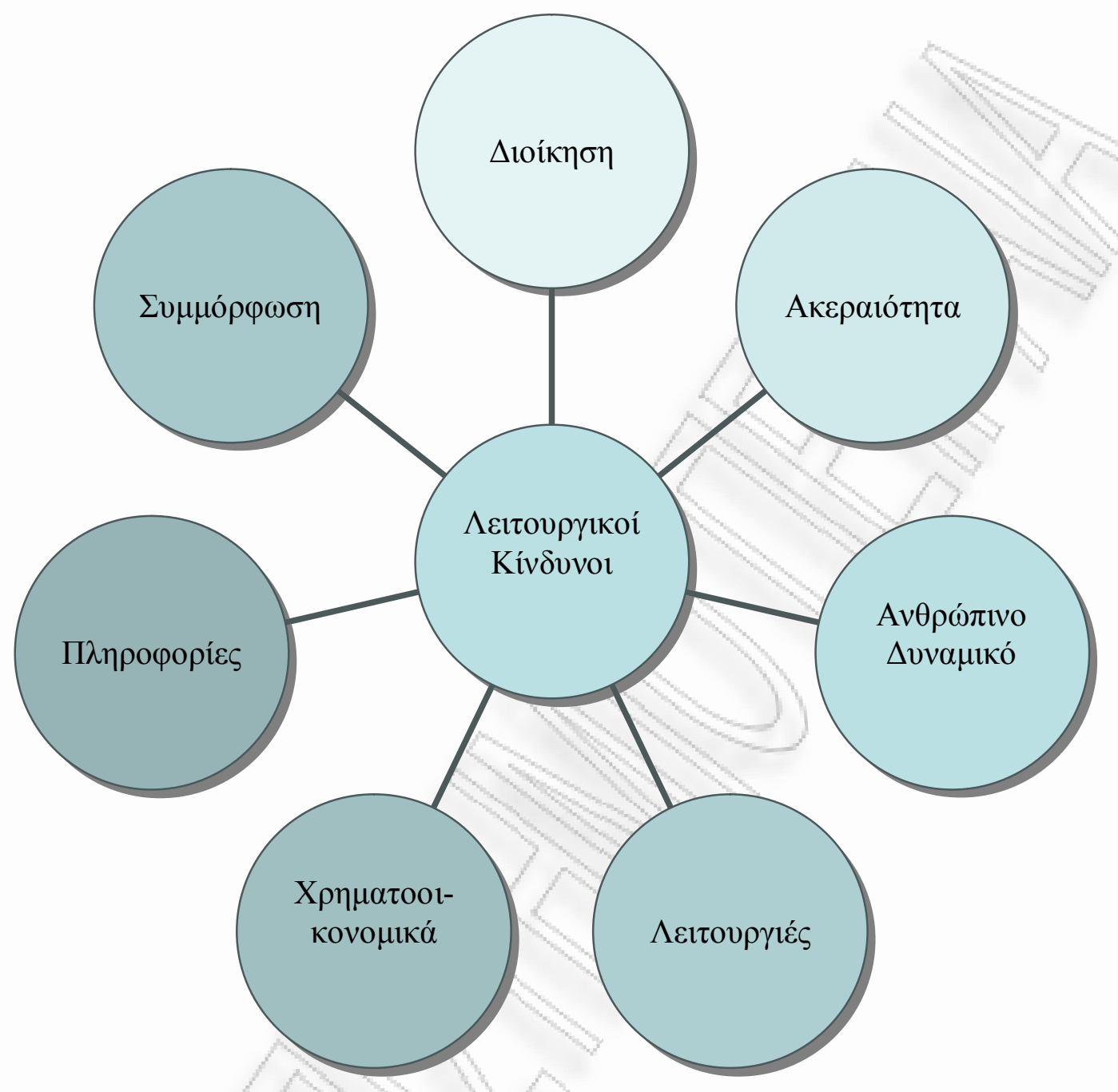

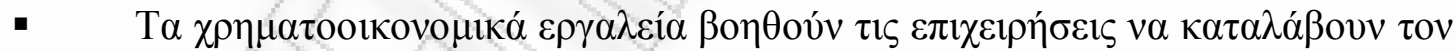

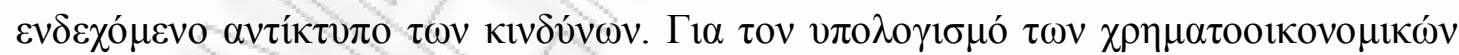

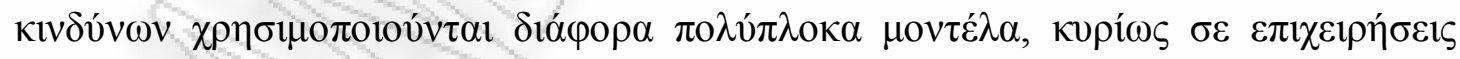

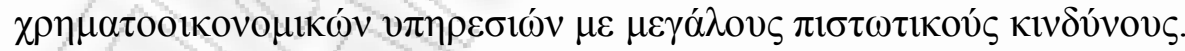




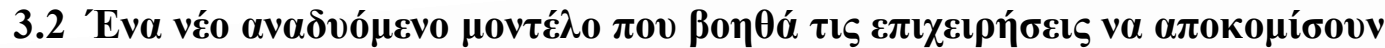

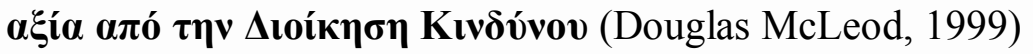

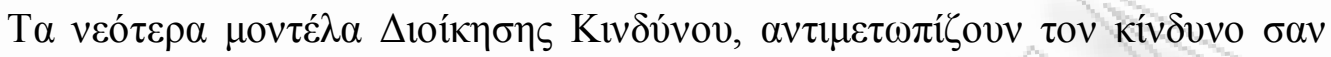

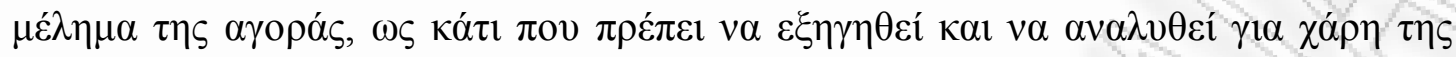

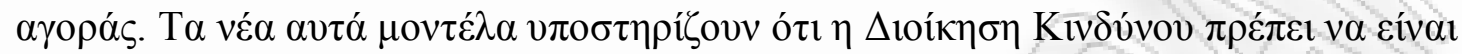

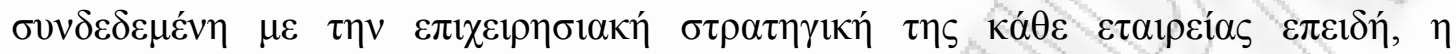

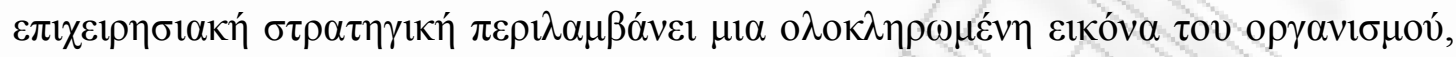

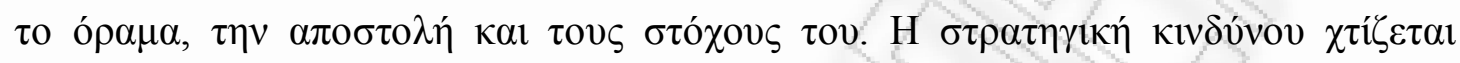

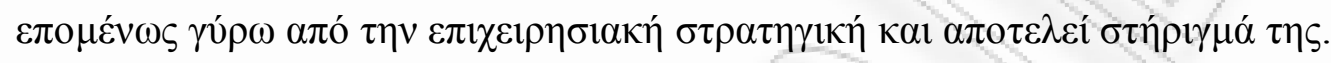

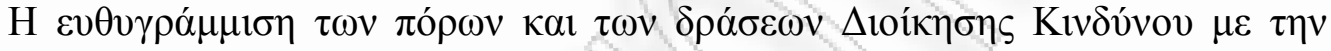

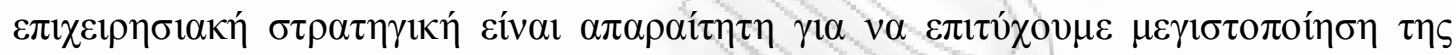

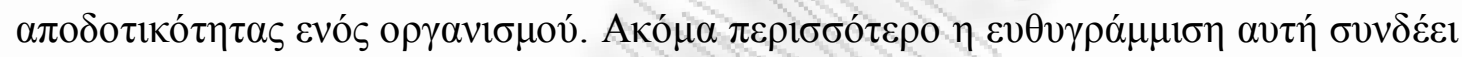

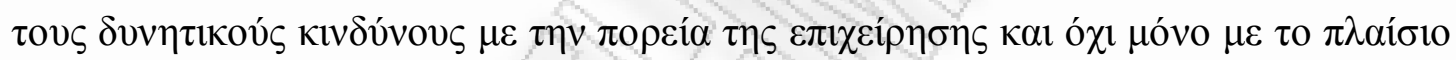

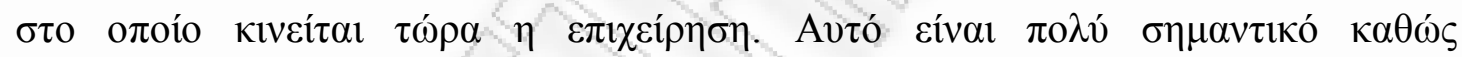

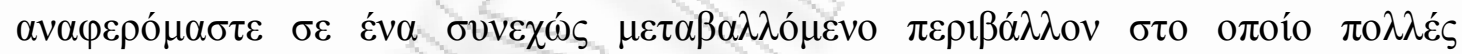

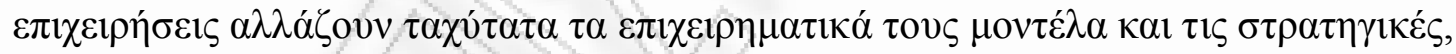

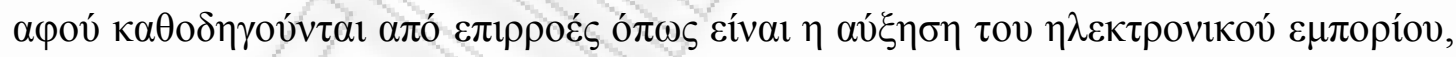

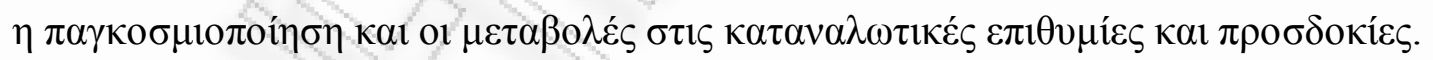

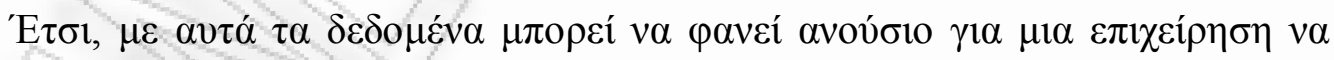

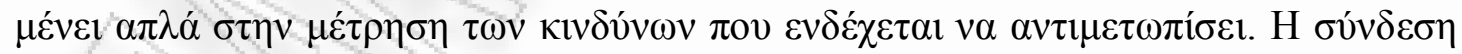

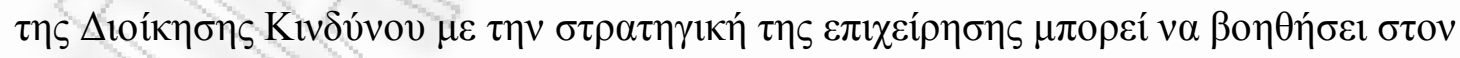

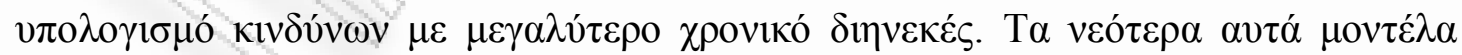

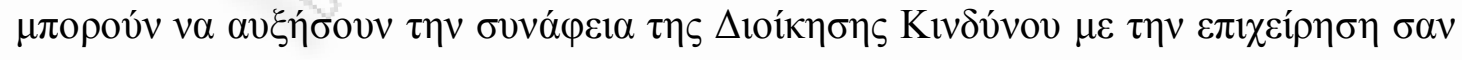
бט́voไo. 


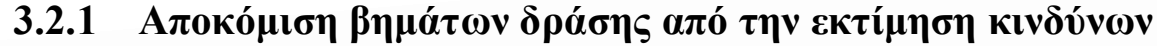

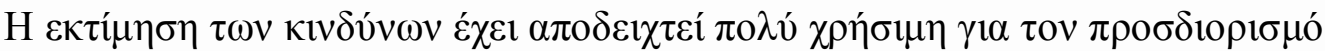

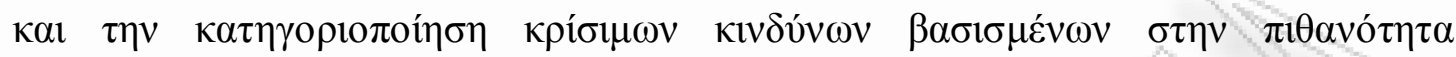

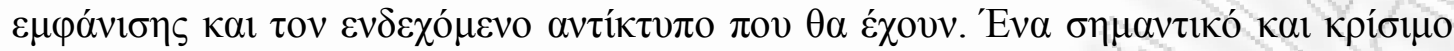

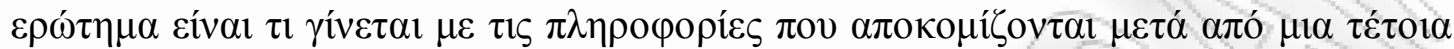

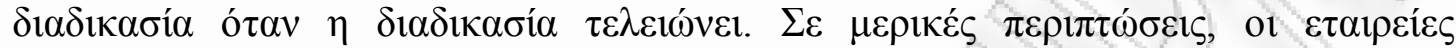

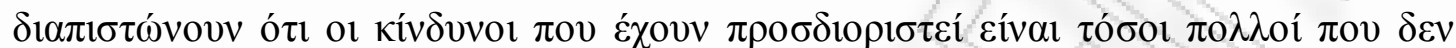

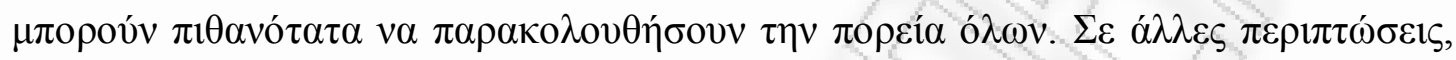

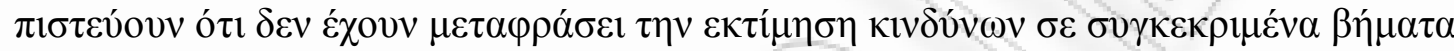

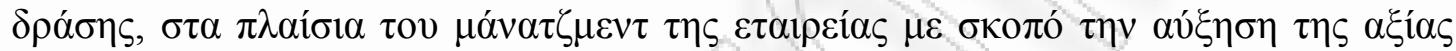
$\tau \eta \varsigma$.

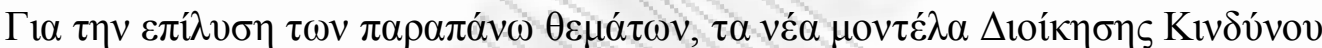

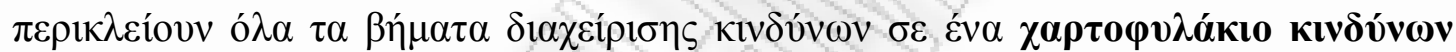

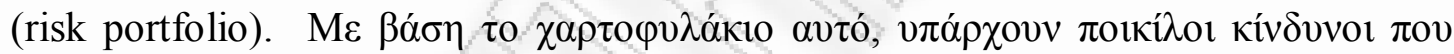

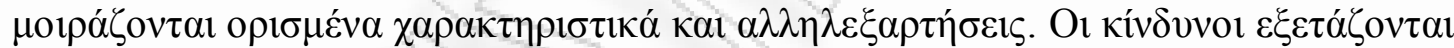

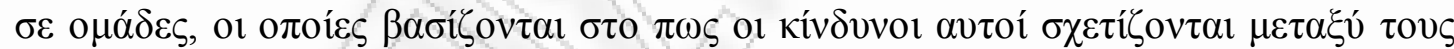

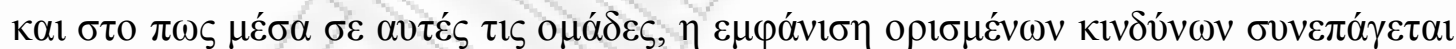

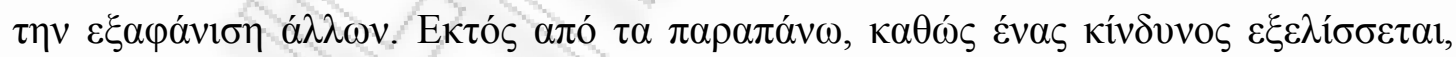

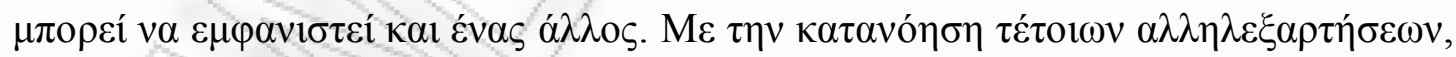

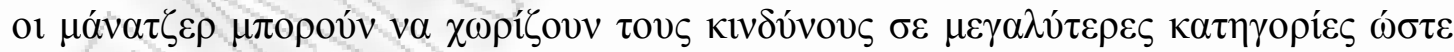

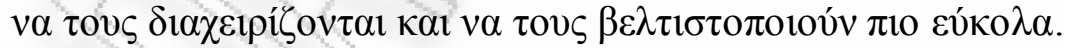

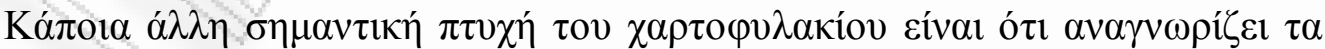

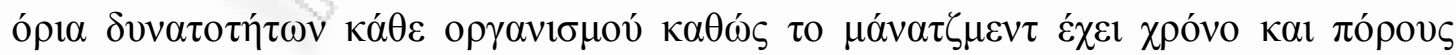

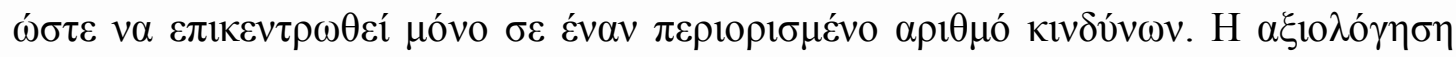

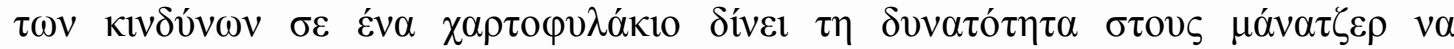




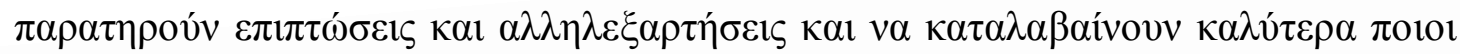

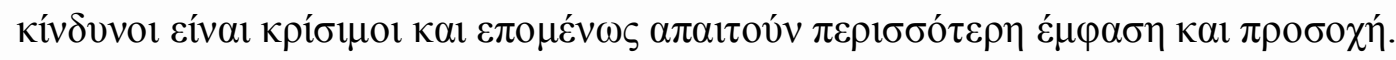

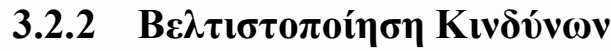

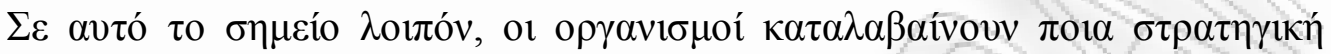

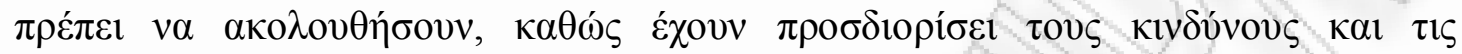

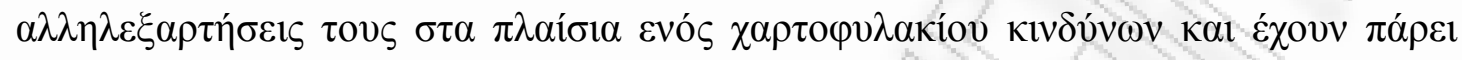

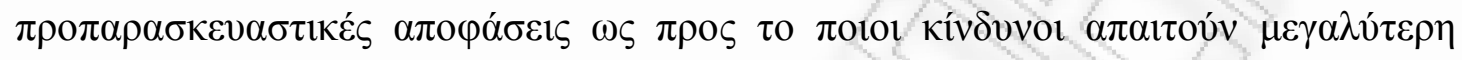

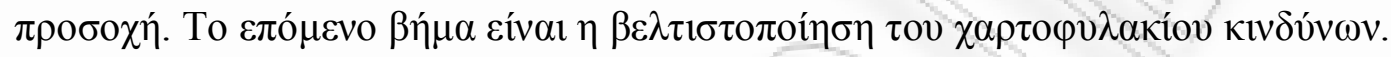

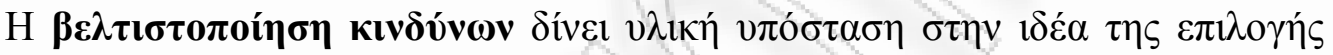

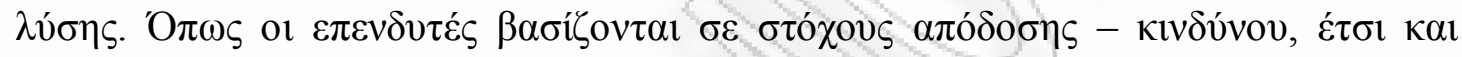

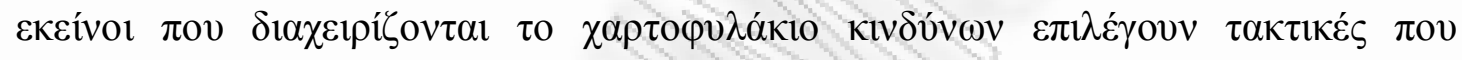

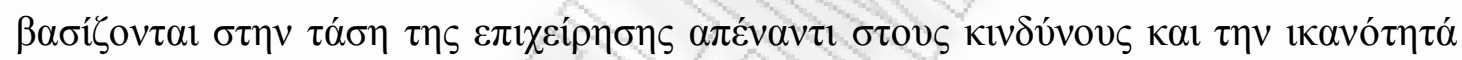

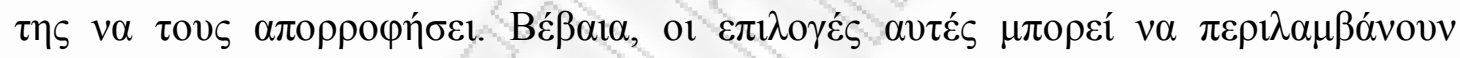

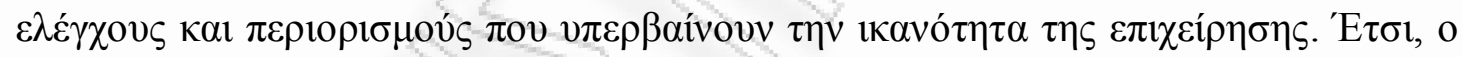

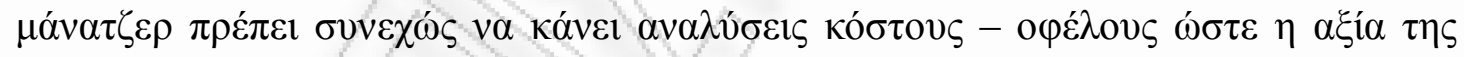

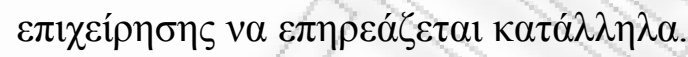

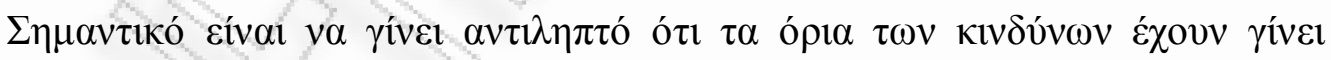

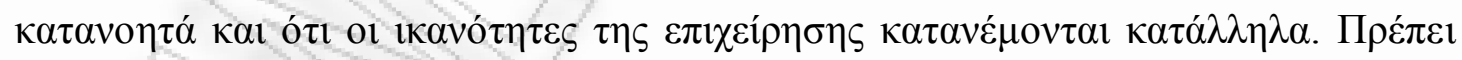

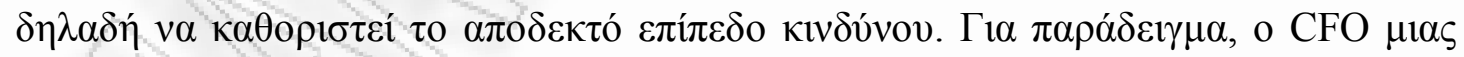

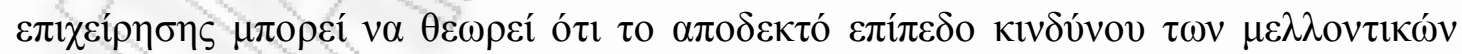

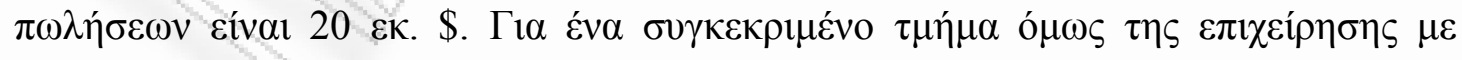

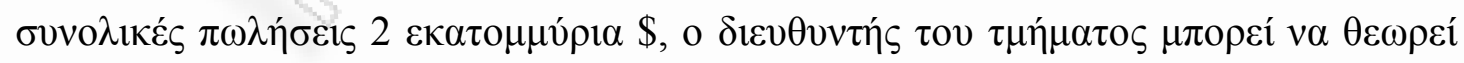

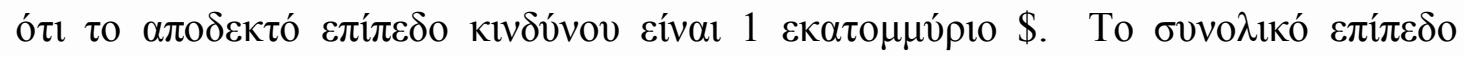




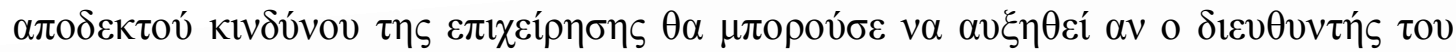

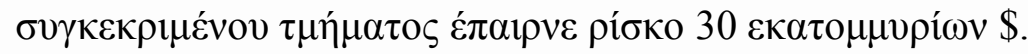

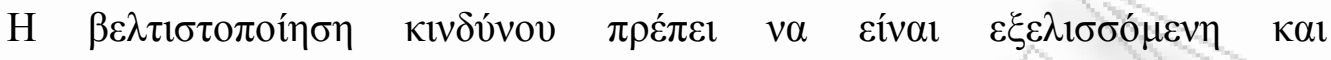

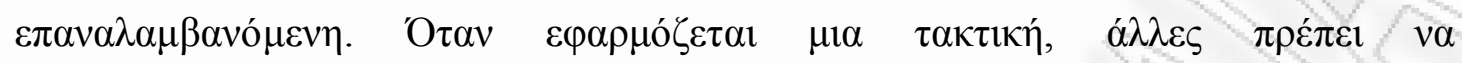

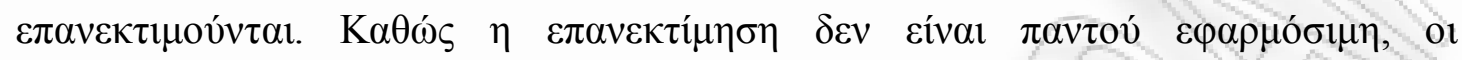

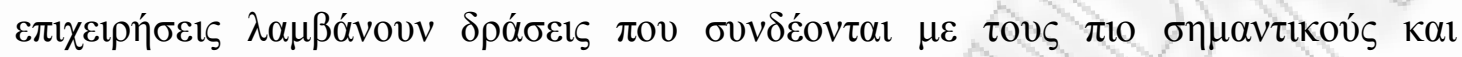

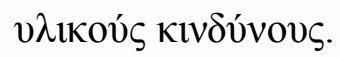

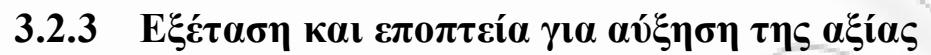

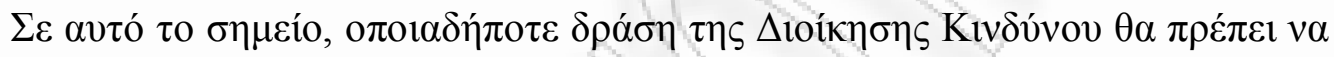

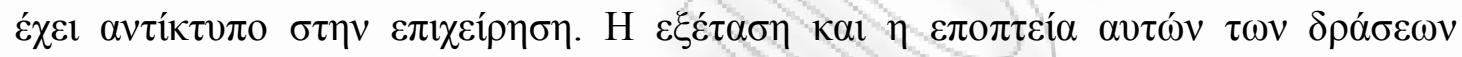

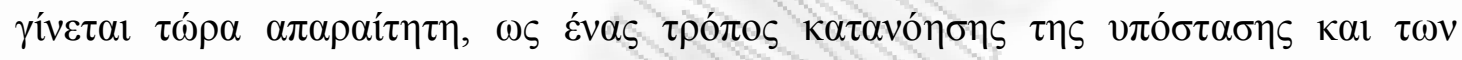

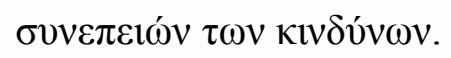

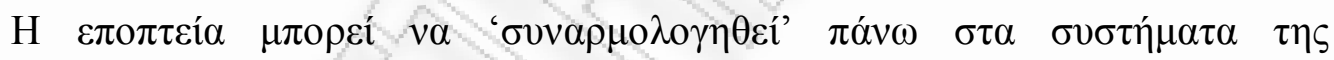

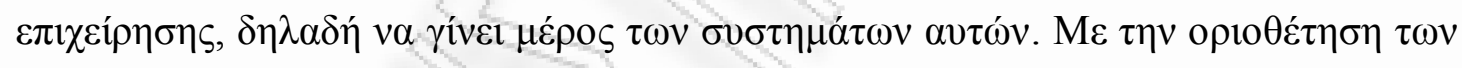

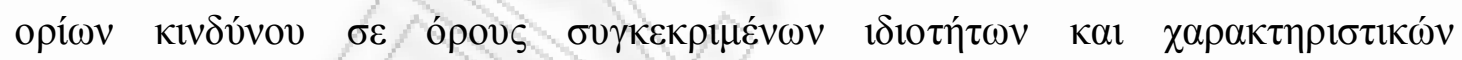

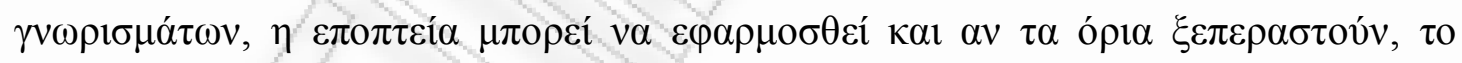

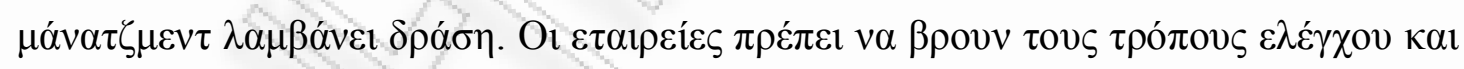

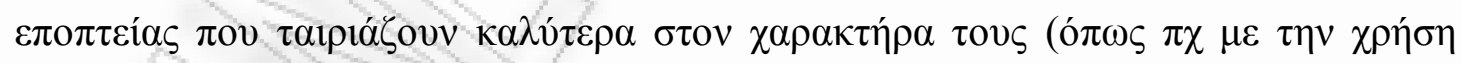

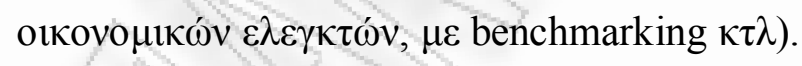

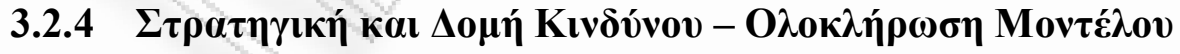

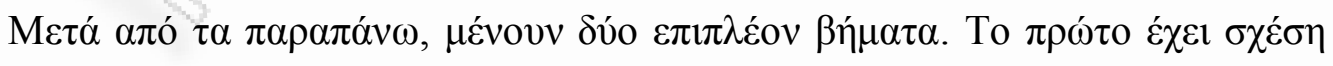

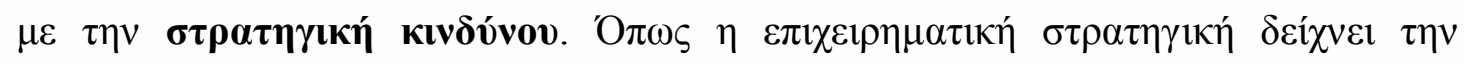

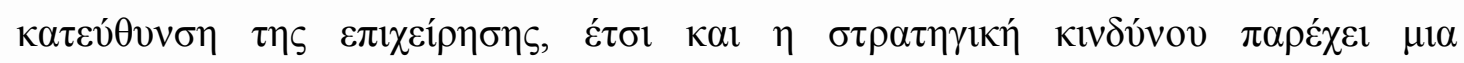




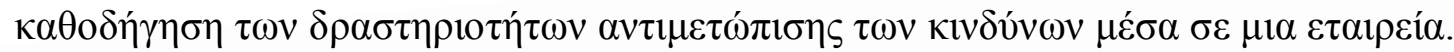

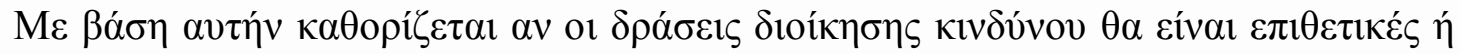

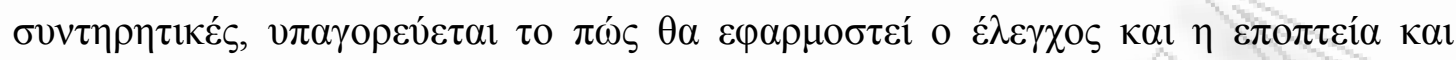

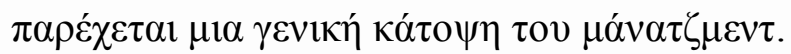

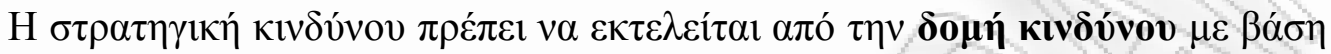

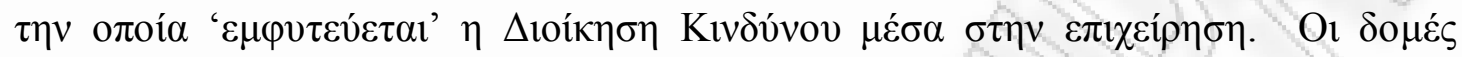

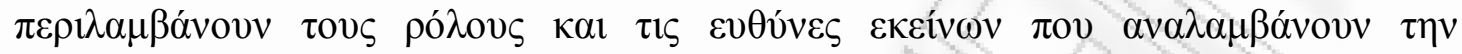

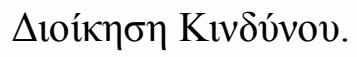

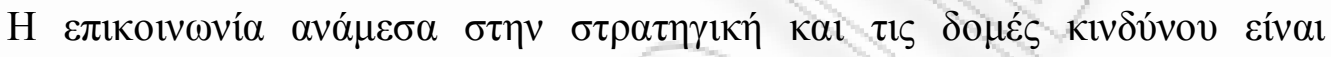

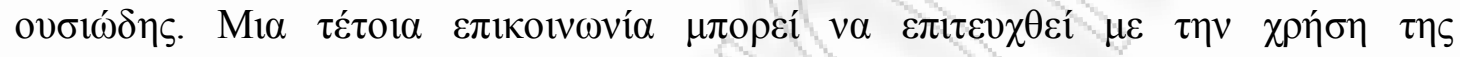

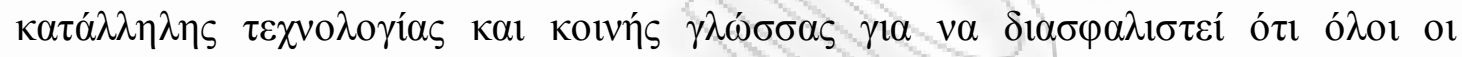

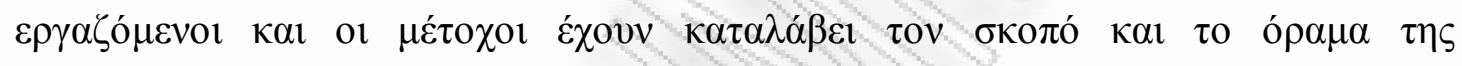

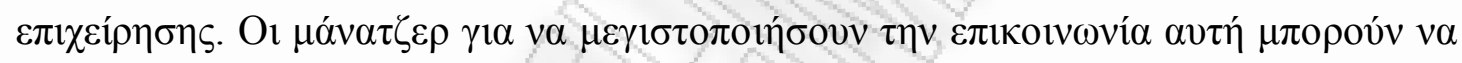

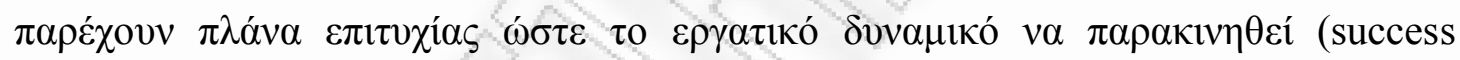

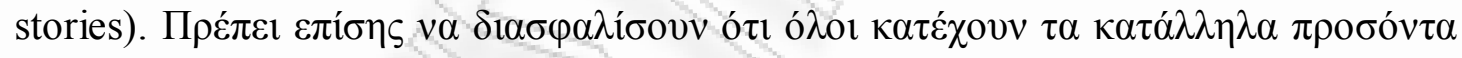

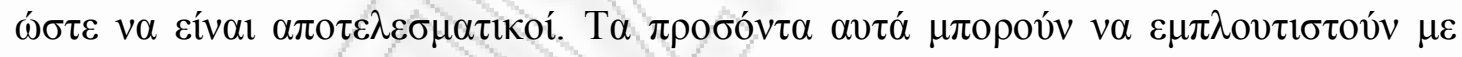

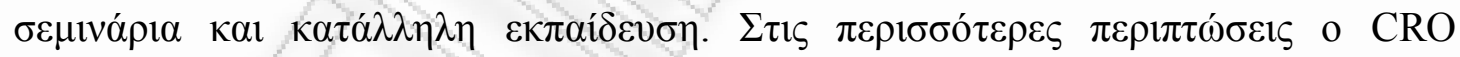

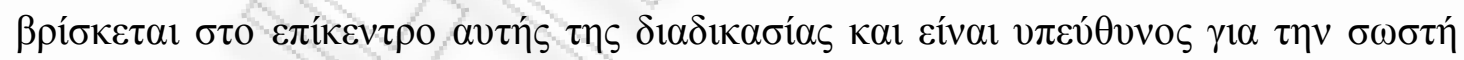

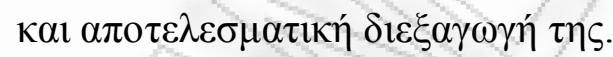

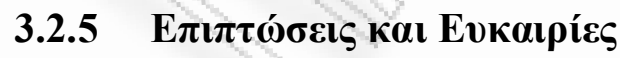

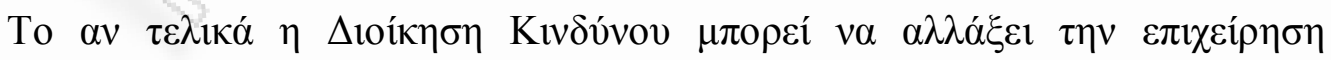

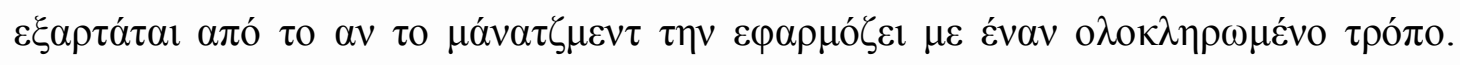

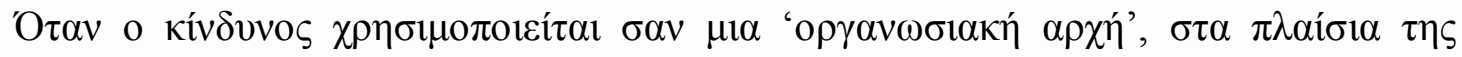




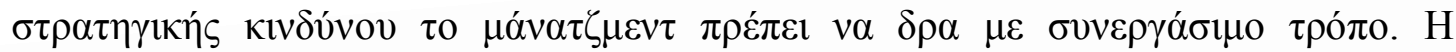

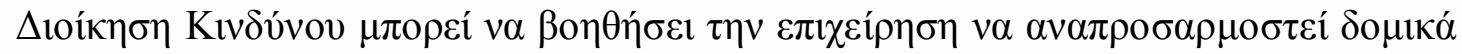

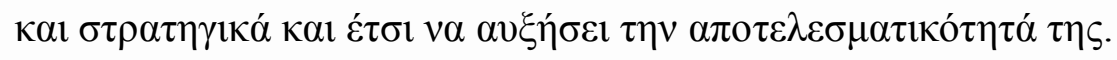

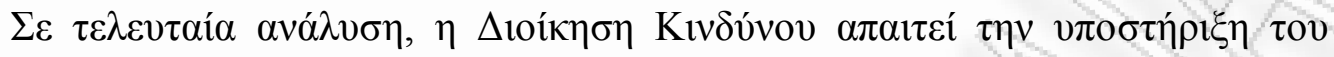

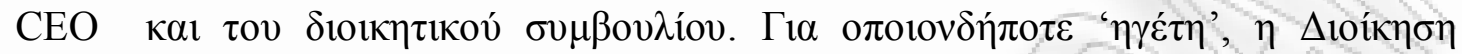

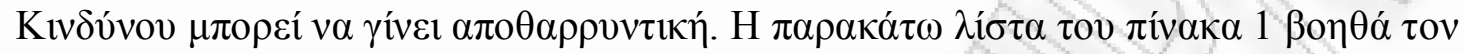

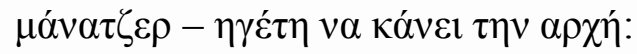

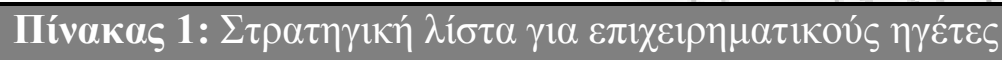

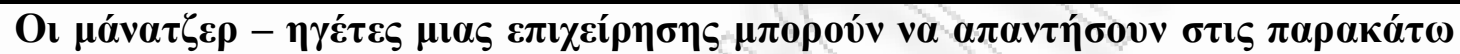

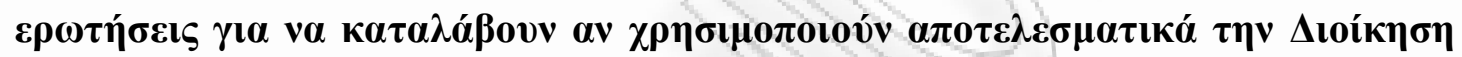

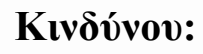

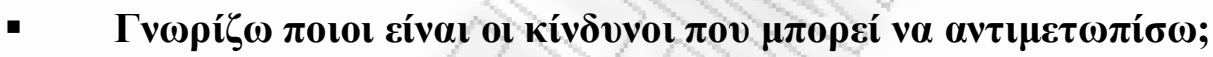

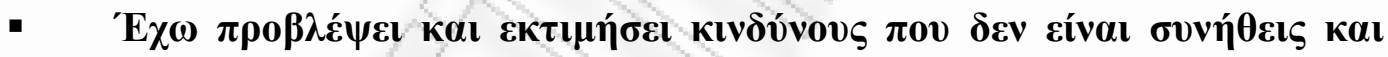

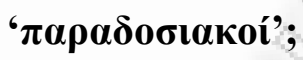

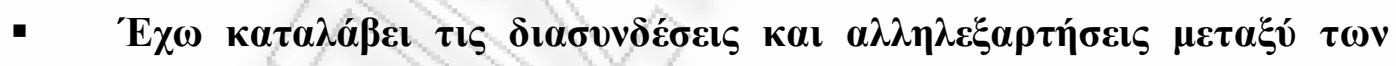

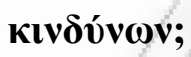

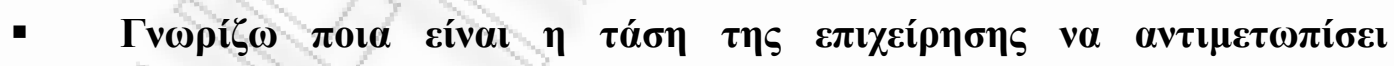
Kıvช์vovs;

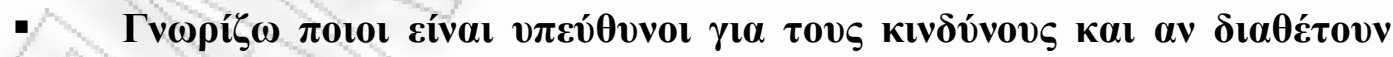

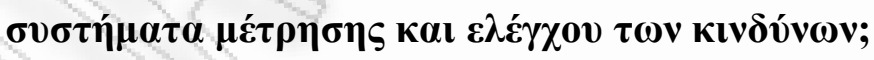

- Y Yó

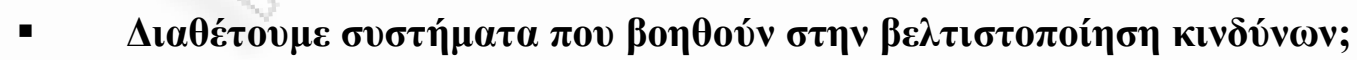

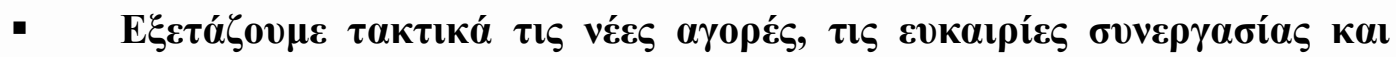

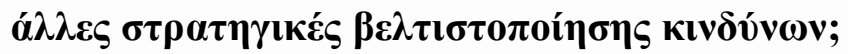




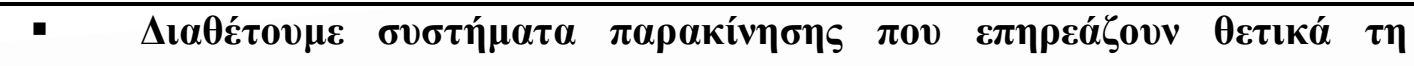

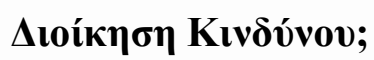

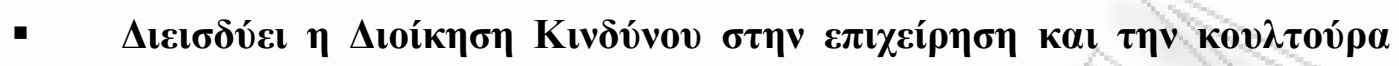
$\tau \eta \varsigma ;$

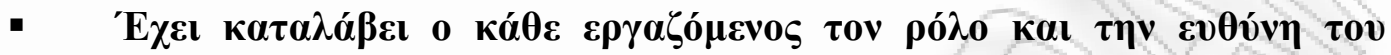

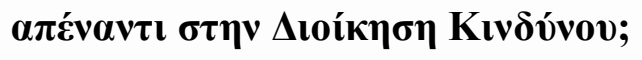

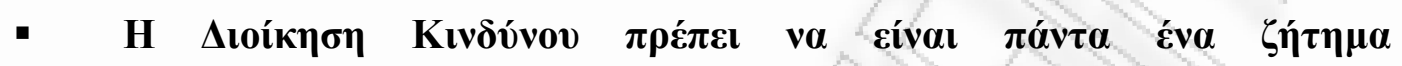

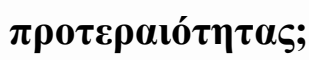

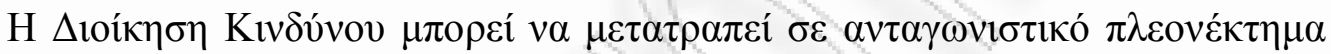

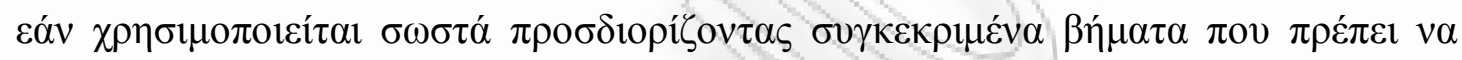

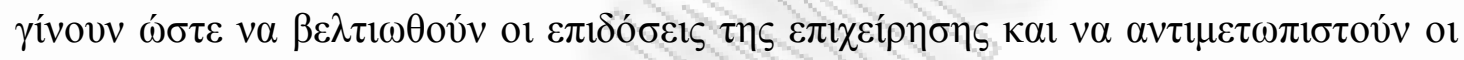

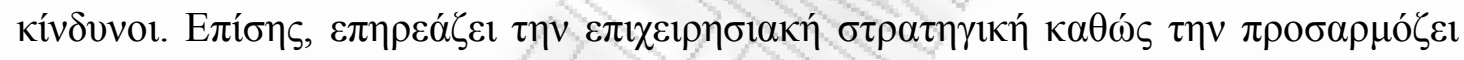

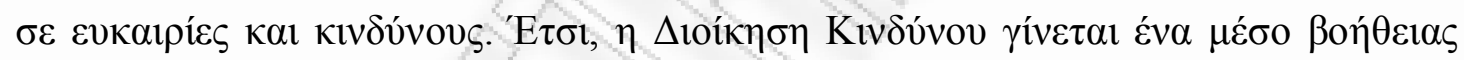

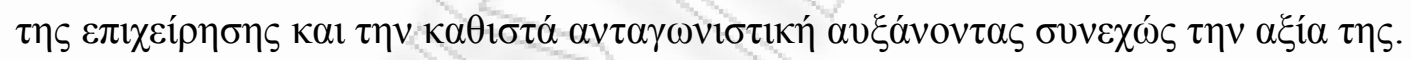

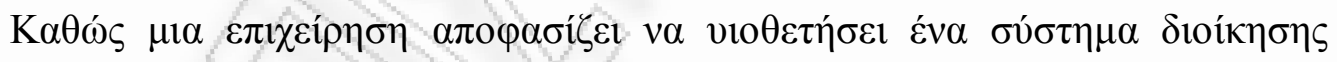

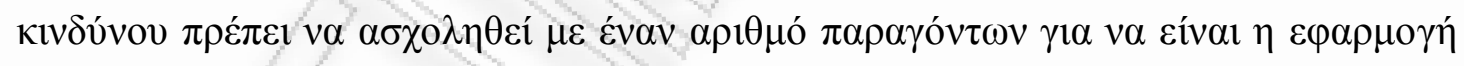

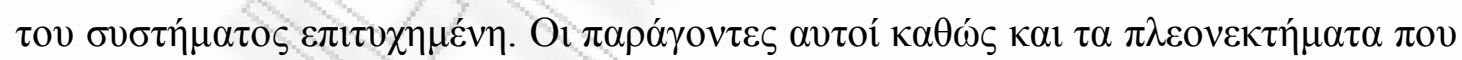

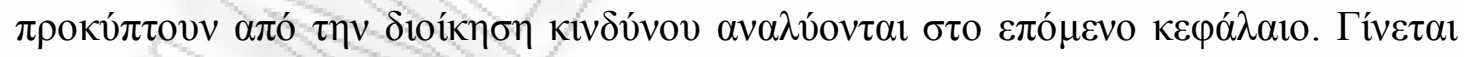

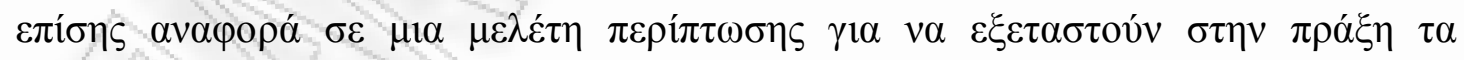
$\pi \alpha \rho \alpha \pi \alpha ́ v \omega$. 


\section{КЕФАААIO 4}

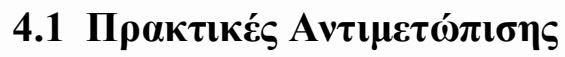

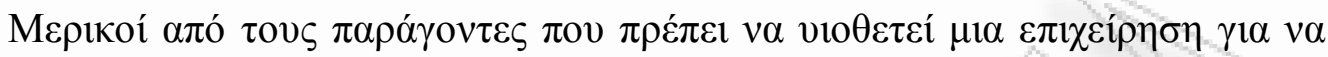

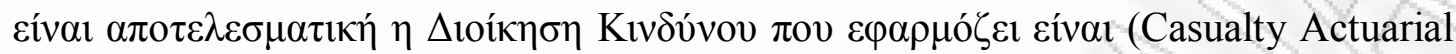
Society):

\section{$\underline{K \alpha \theta o \rho l \sigma \mu o ́ \varsigma ~ \tau o v ~ ' v \pi \varepsilon ́ \rho \mu \alpha \chi o v ' ~} \eta \eta \varsigma \Delta l o i k \eta \sigma \eta \varsigma K l v \delta \dot{v v o v}$}

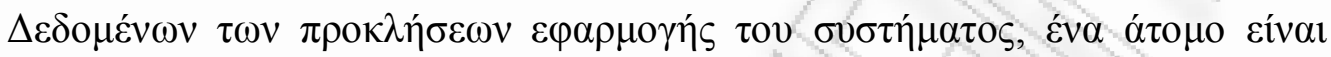

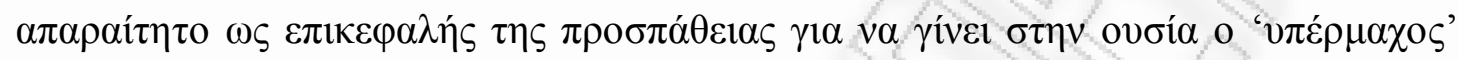

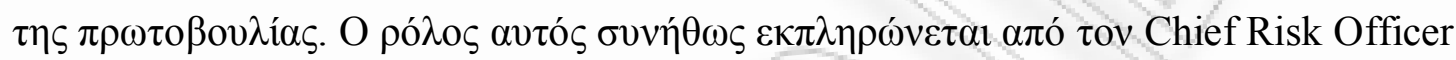

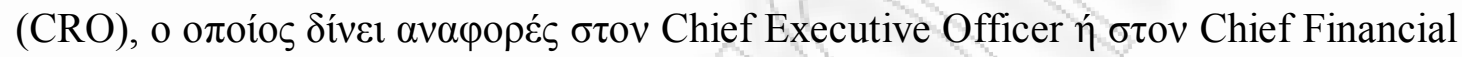

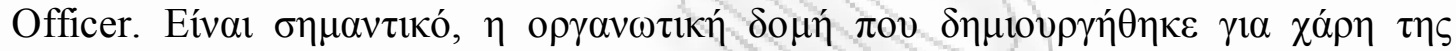

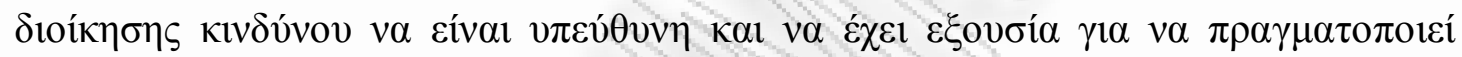

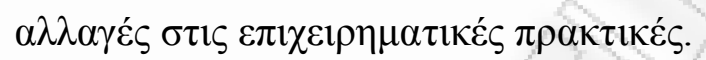

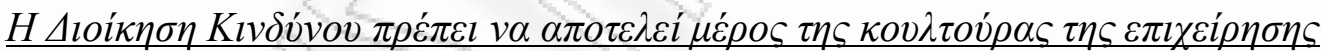

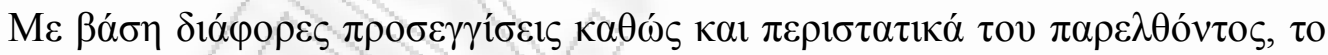

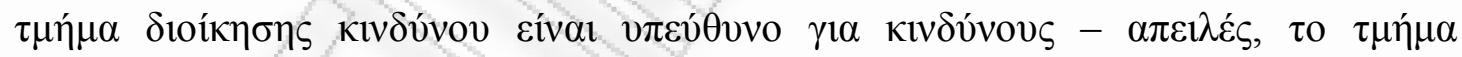

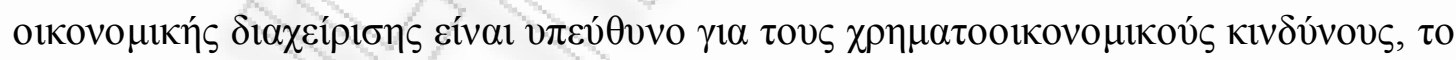

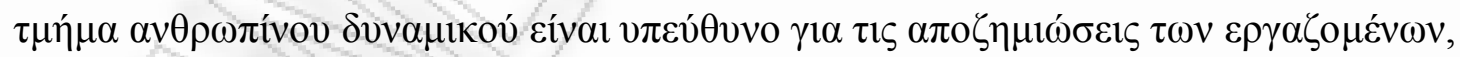

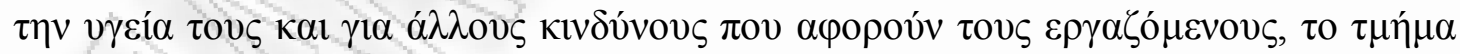

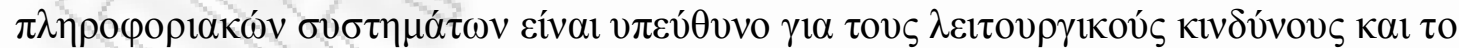

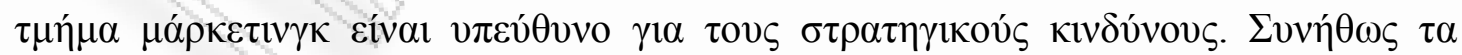

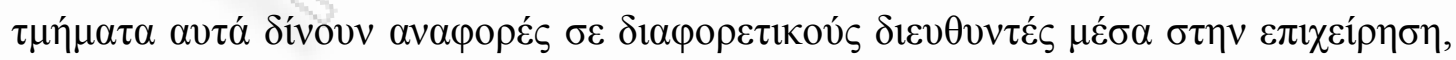

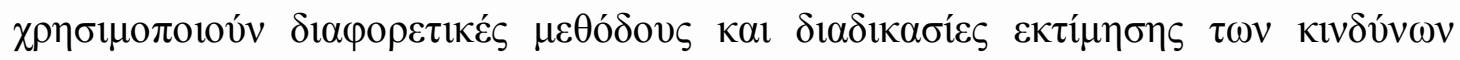

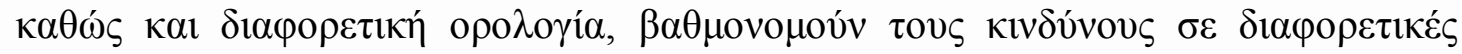




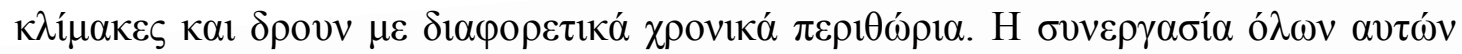

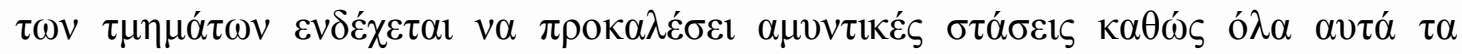

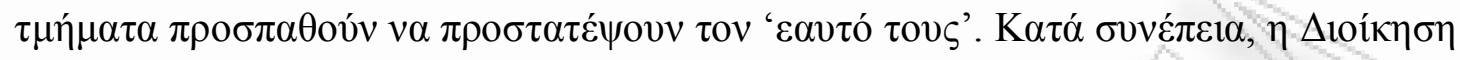

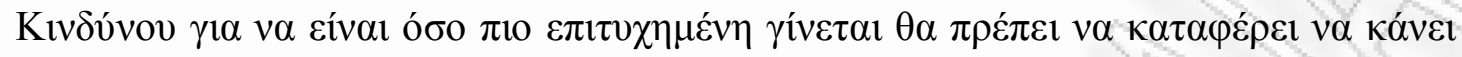

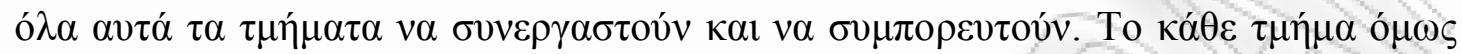

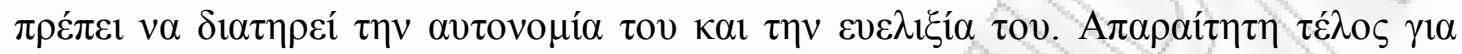

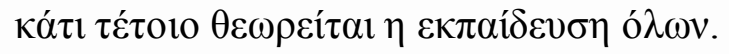

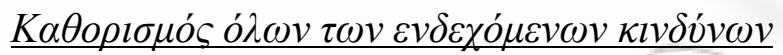

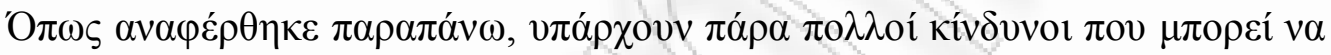

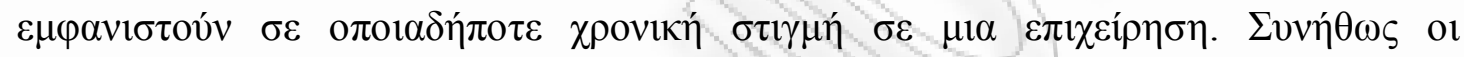

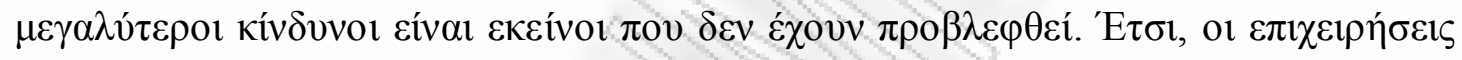

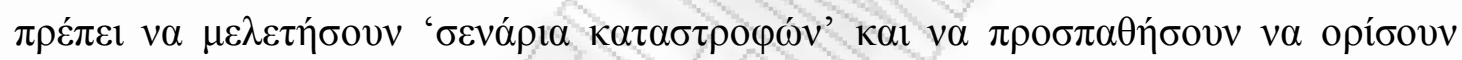

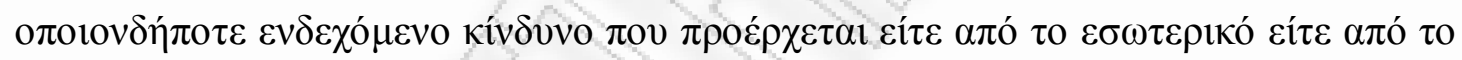

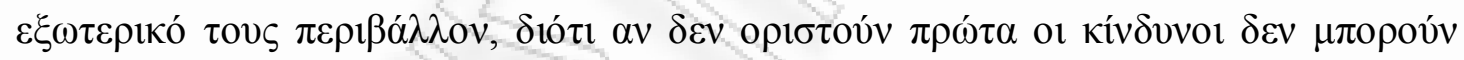

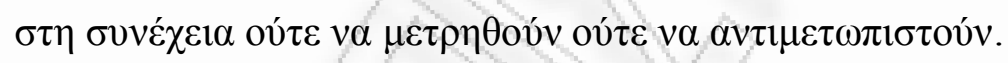

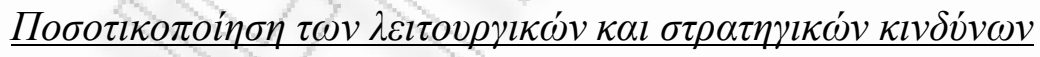

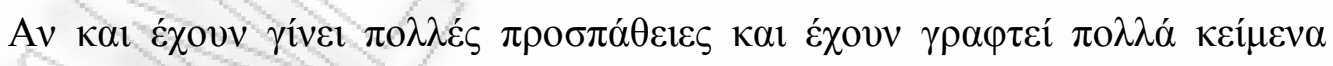

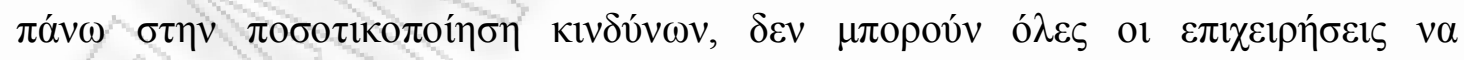

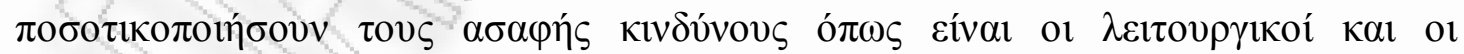

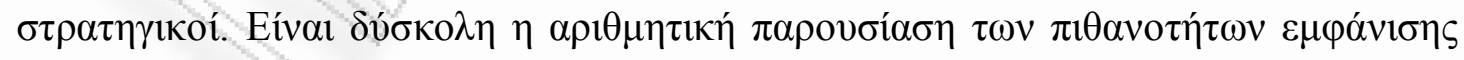

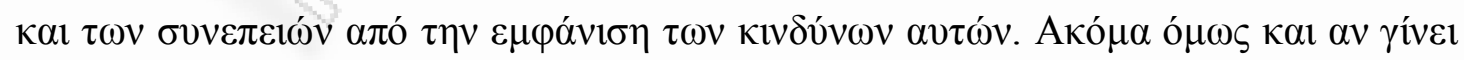

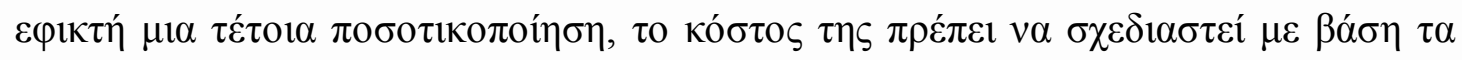

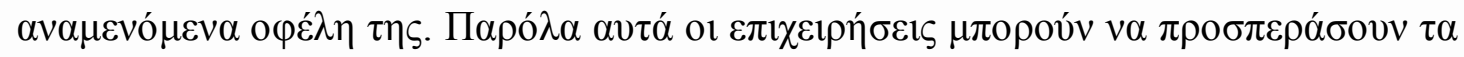




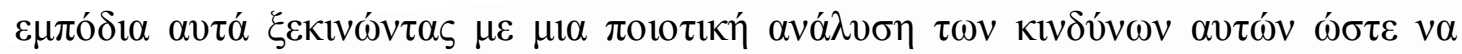

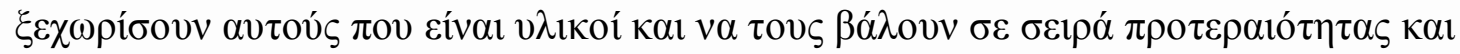

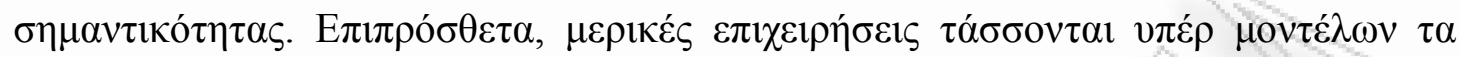

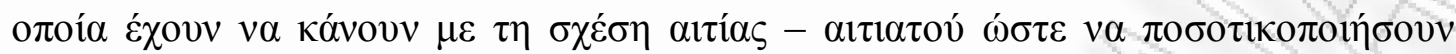

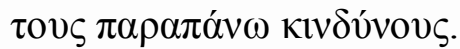

\section{$\underline{H \varepsilon v \sigma \omega \mu \alpha \dot{\alpha} \tau \omega \sigma \eta \tau \omega \nu \kappa l v \delta \dot{v} v \omega v}$}

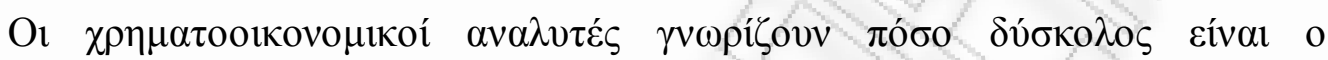

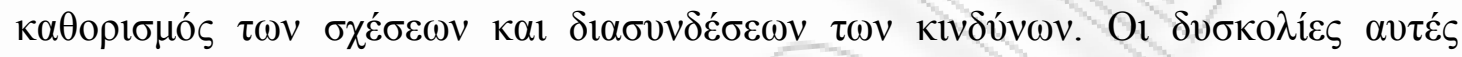

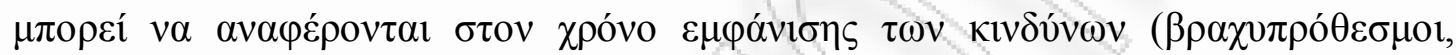

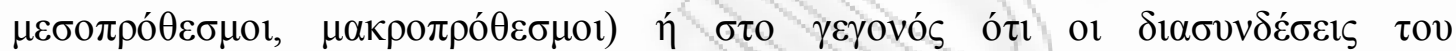

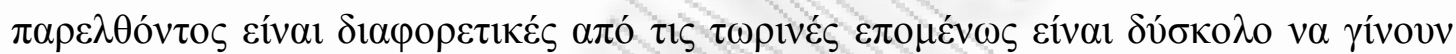

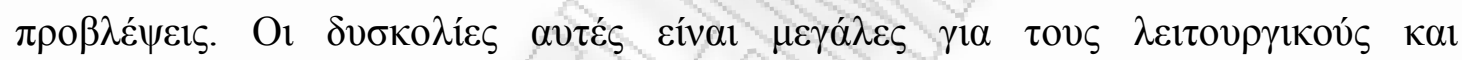

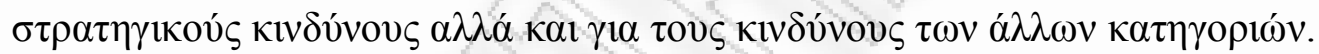

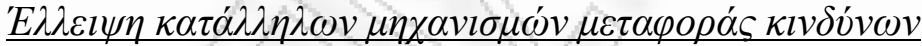

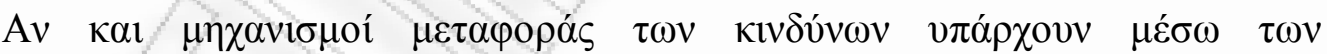

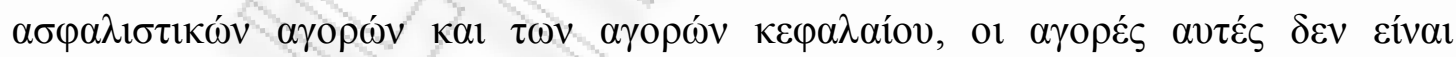

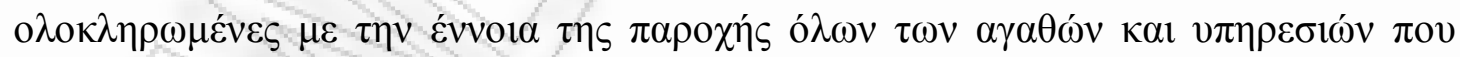

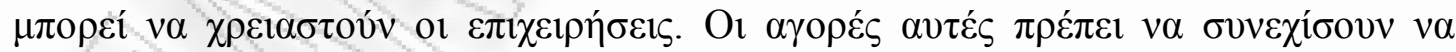

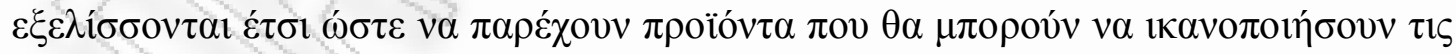
$\alpha v \alpha ́ \gamma \kappa \varepsilon \varsigma \tau \omega \nu \varepsilon \pi \imath \chi \varepsilon \iota p \eta \sigma \varepsilon \omega \nu$. 


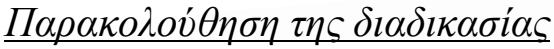

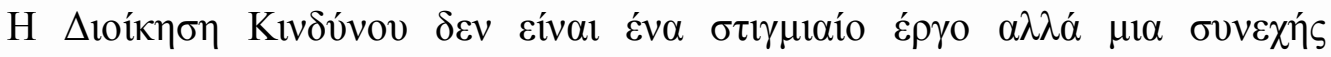

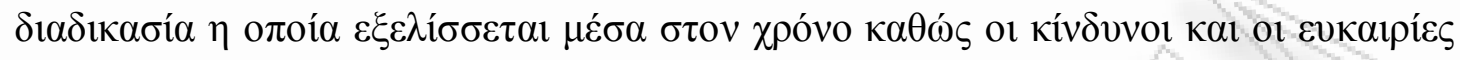

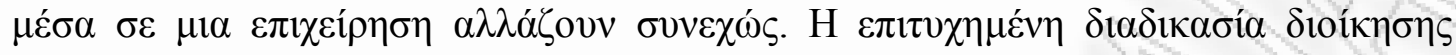

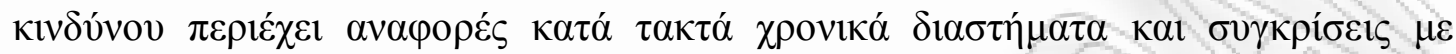

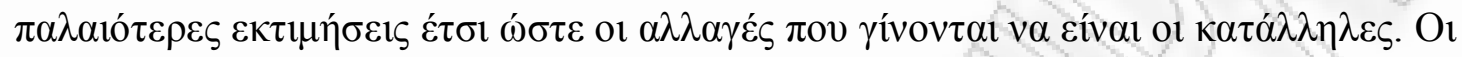

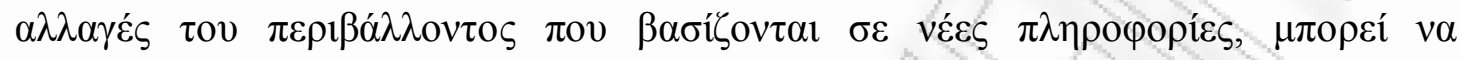

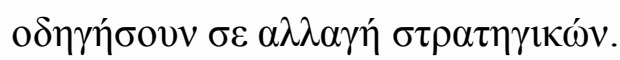

\section{$\underline{\Sigma v \gamma \kappa \rho \alpha \tau \eta \mu \varepsilon ́ v o ~ \xi ̌ \kappa i ́ v \eta \mu \alpha ~}$}

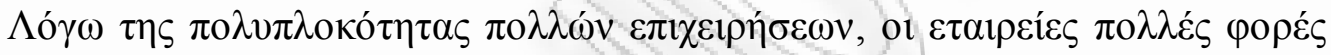

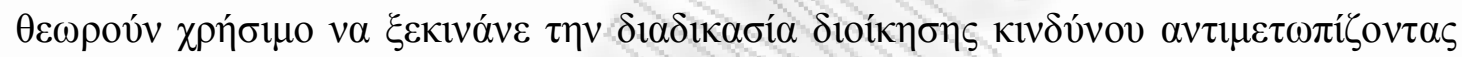

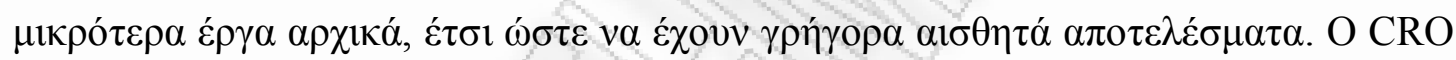

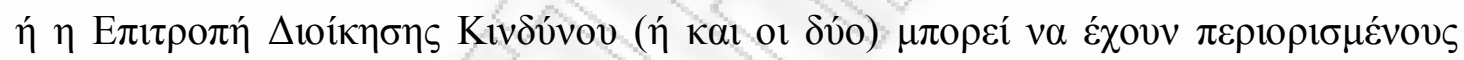

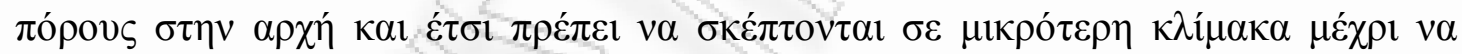

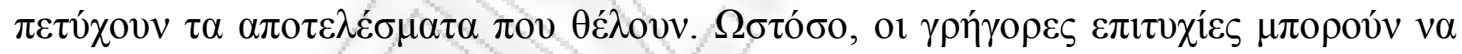

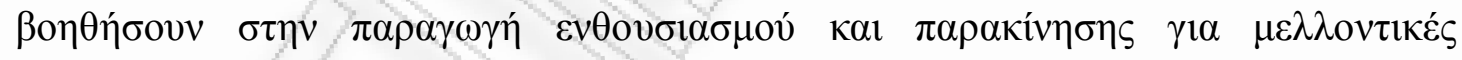

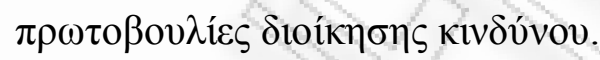

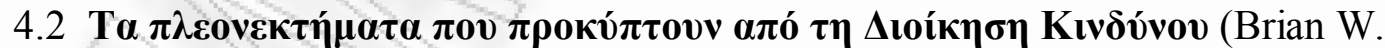

Nocco and Rene M. Stulz, 2006)

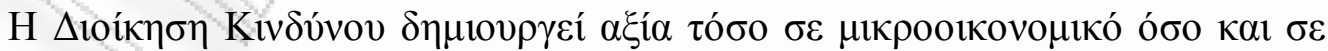

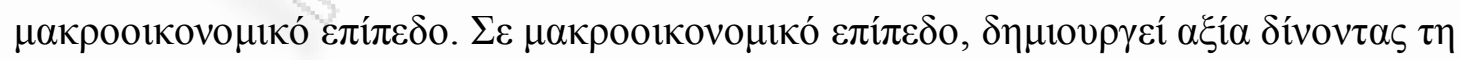

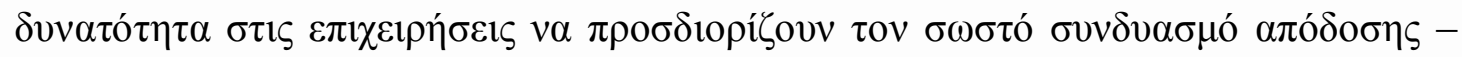

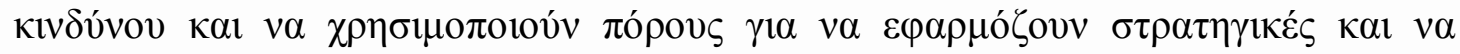




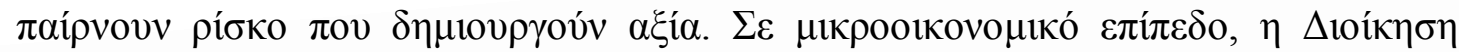

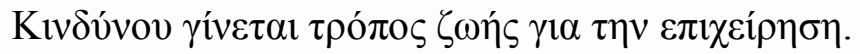

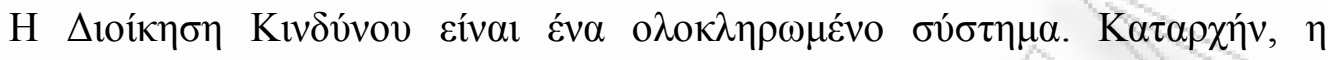

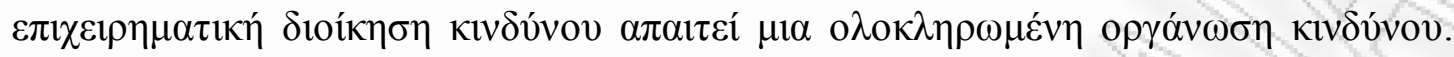

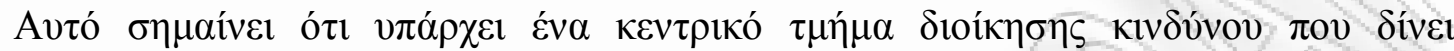

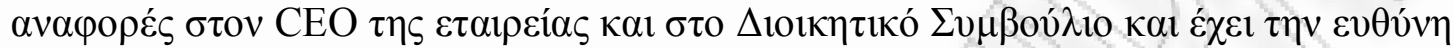

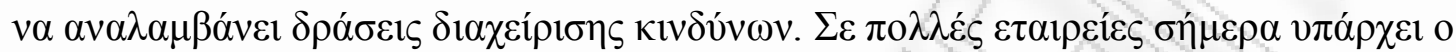

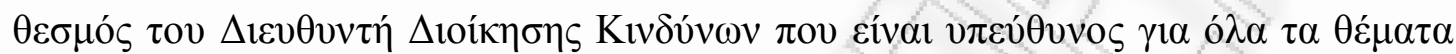

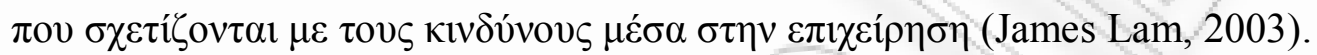

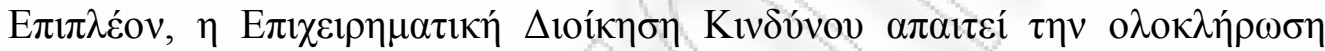

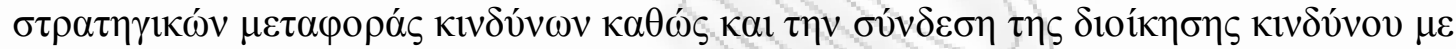

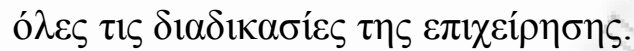

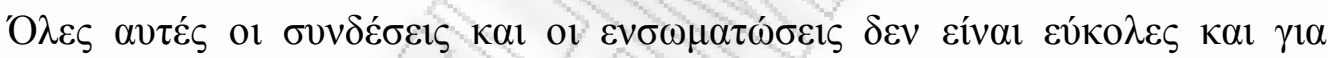

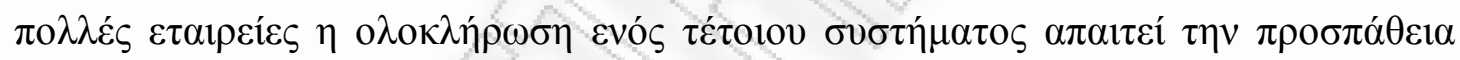

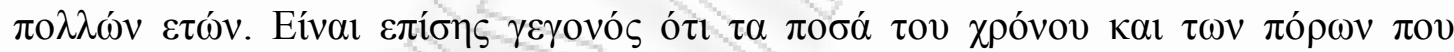

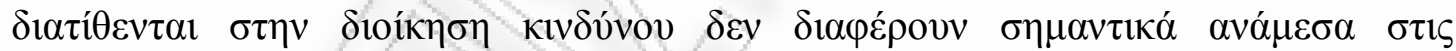

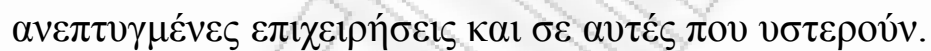

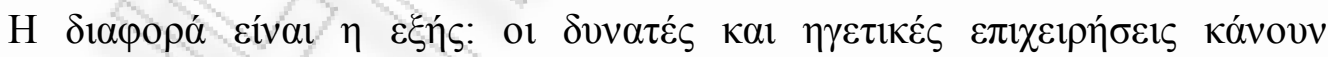

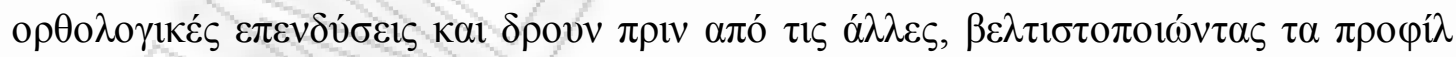

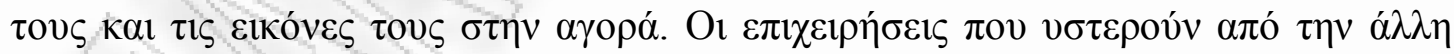

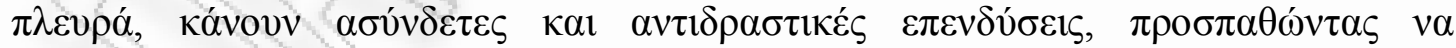

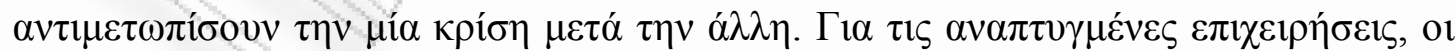

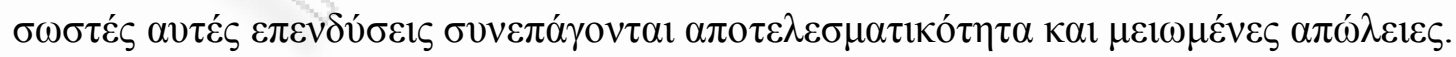

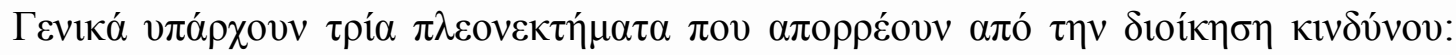




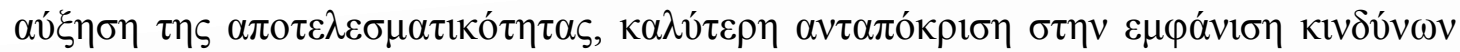

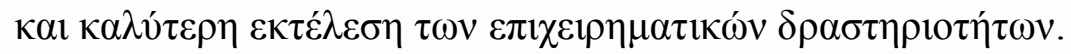

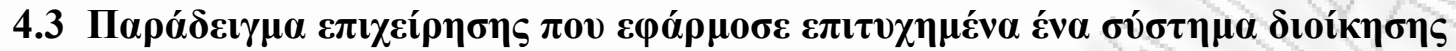

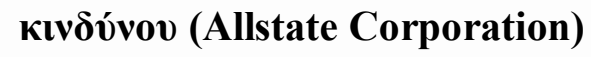

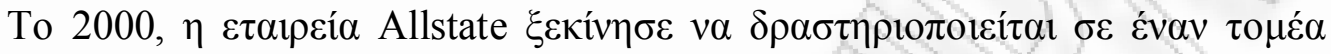

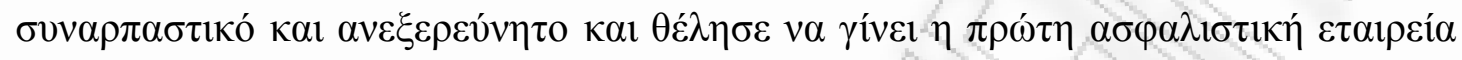

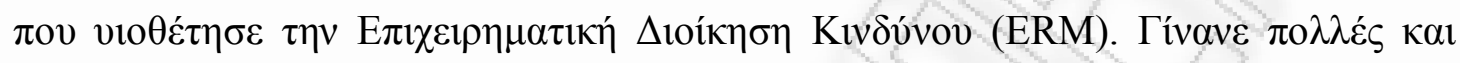

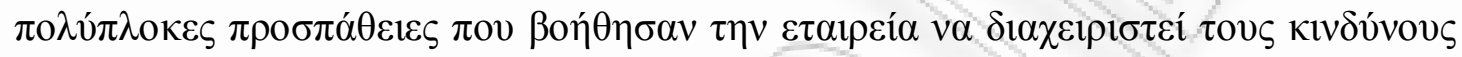

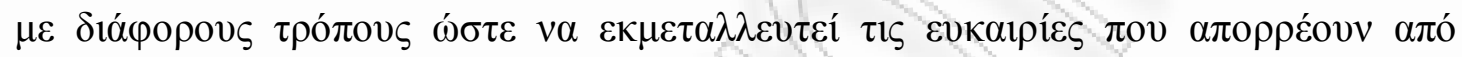

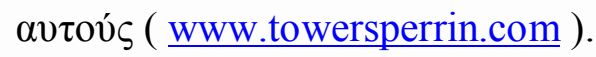

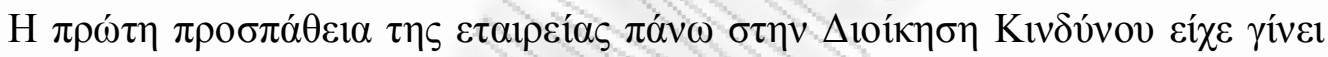

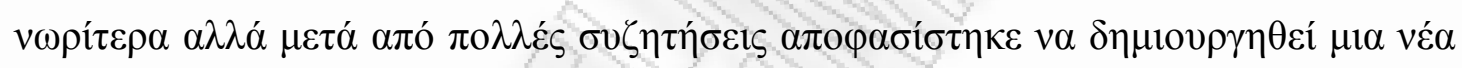

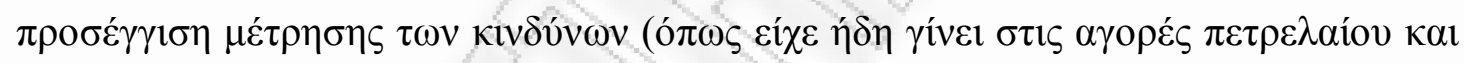
acpíov).

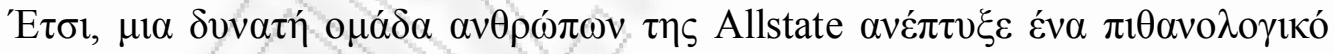

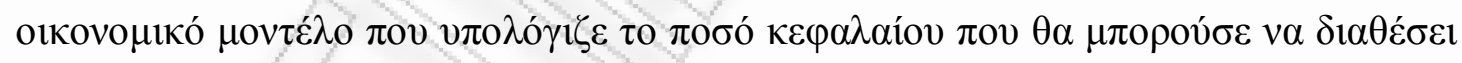

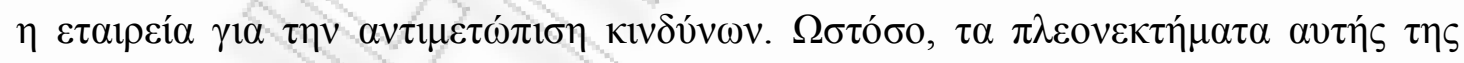

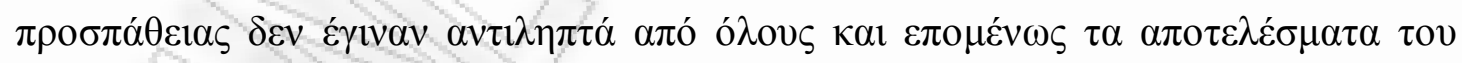

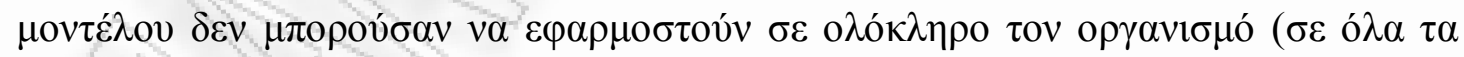

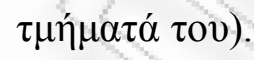

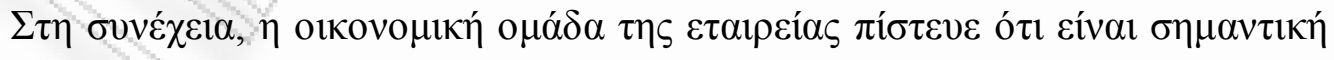

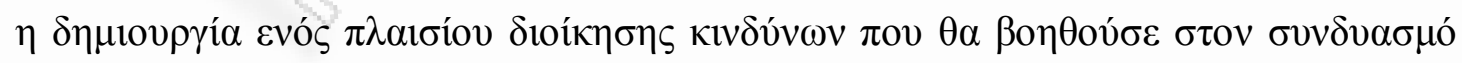

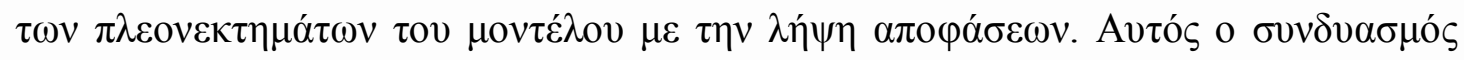

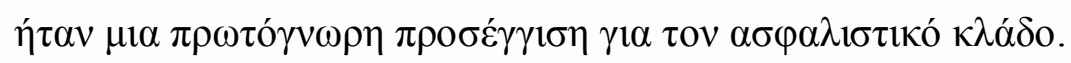




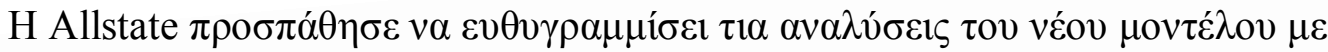

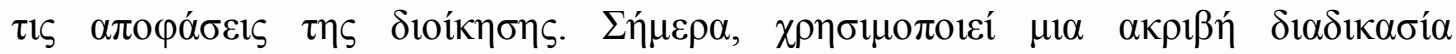

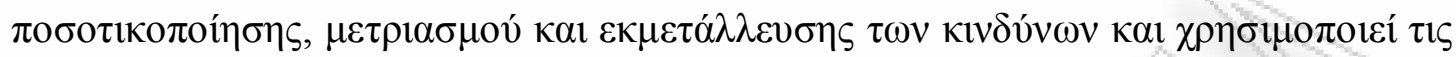

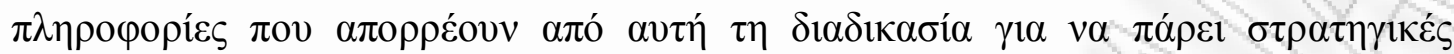

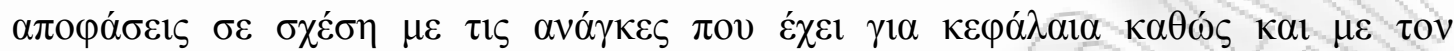

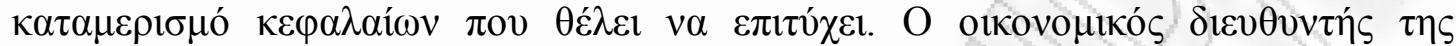

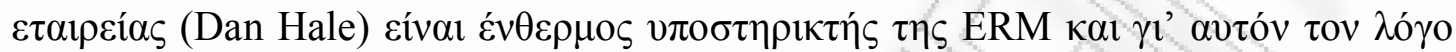

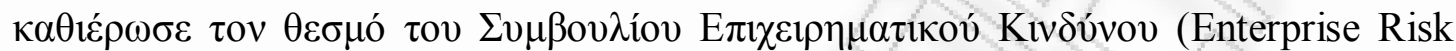

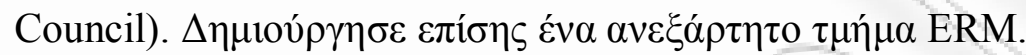

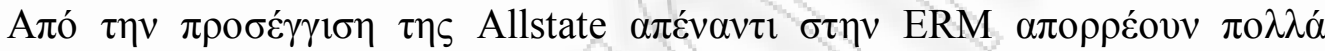

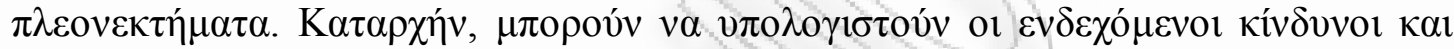

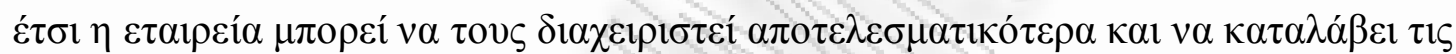

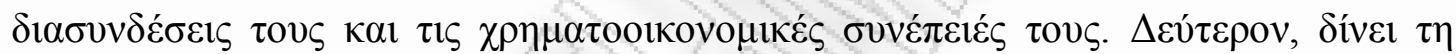

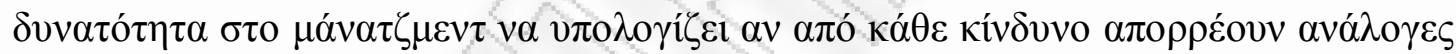

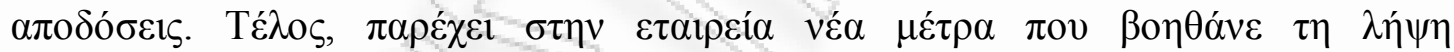

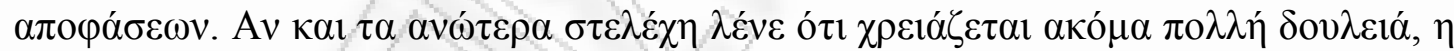

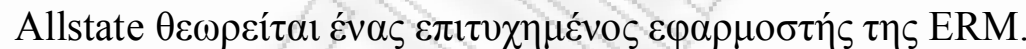

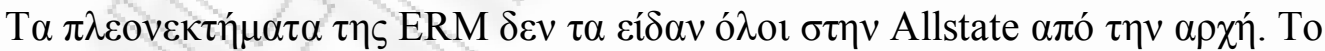

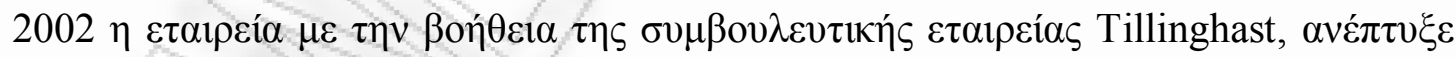

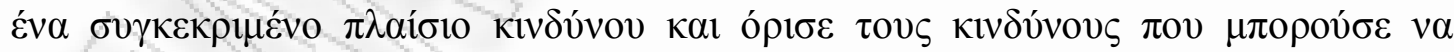

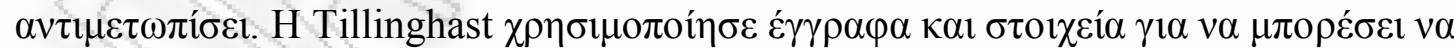

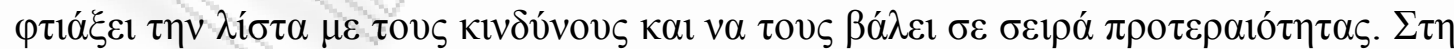

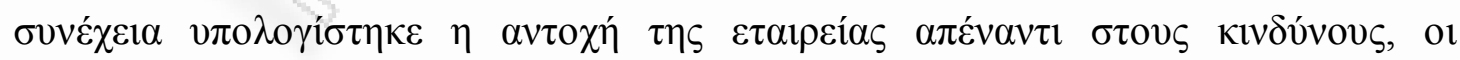

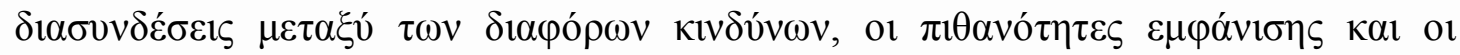

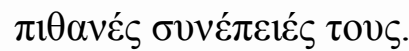




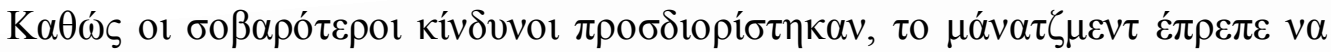

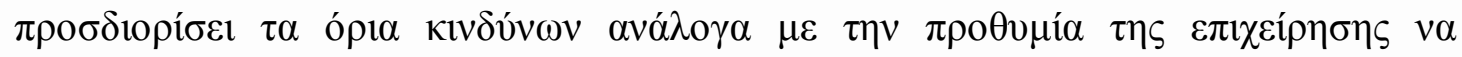
$\varepsilon \pi \varepsilon v \delta \dot{\sigma \varepsilon l ~ \kappa \varepsilon \varphi \alpha ́ \lambda \alpha 1 \alpha ~ \gamma l \alpha ~ \sigma v \gamma \kappa \varepsilon \kappa \rho \mu \varepsilon ́ v o v \varsigma ~ \kappa ı v \delta ์ v o v \varsigma . ~}$

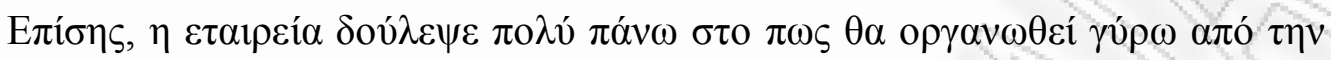

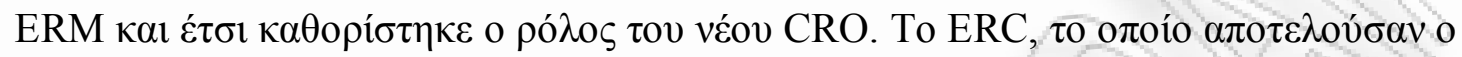

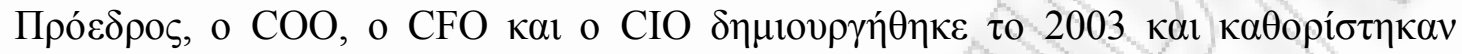

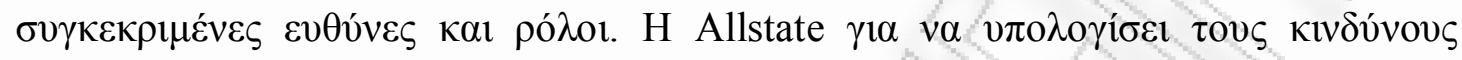

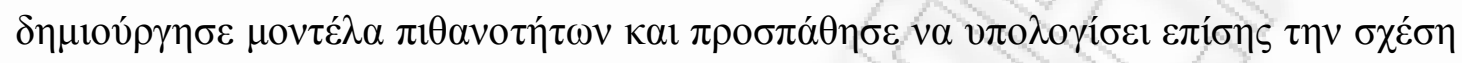
$\alpha \imath i ́ \alpha \varsigma-\alpha \iota \tau \imath \alpha \tau o v ́$. 


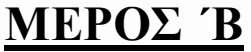

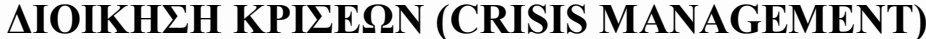

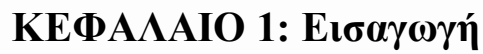

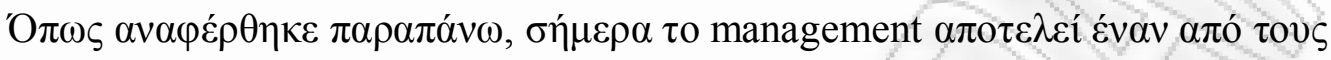

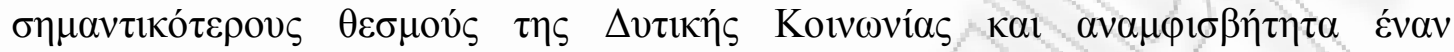
$\alpha \pi \alpha \rho \alpha i ́ \tau \eta \tau o ~ \sigma v v \tau \varepsilon \lambda \varepsilon \sigma \tau \eta ́ ~ \pi \alpha \rho \alpha \gamma \omega \gamma \eta ́ s$.

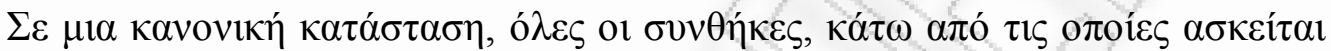

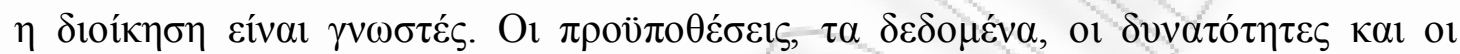

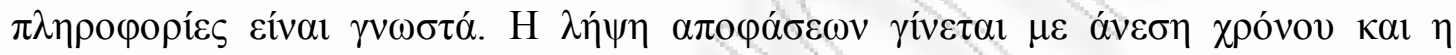

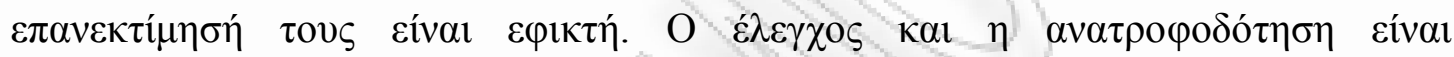

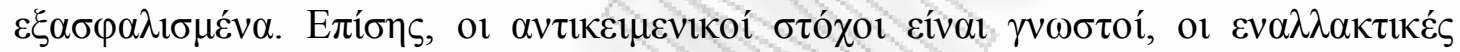

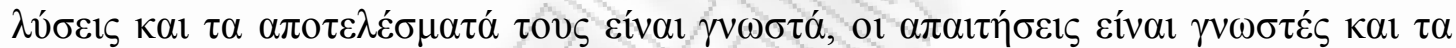

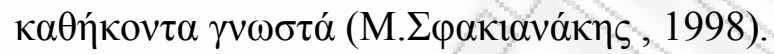

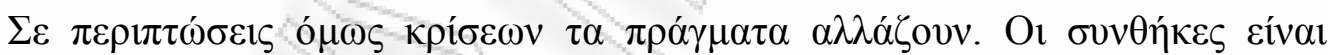

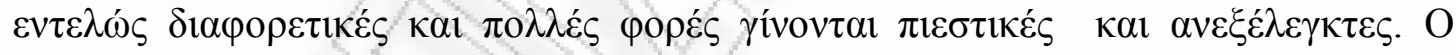

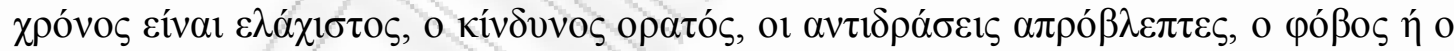

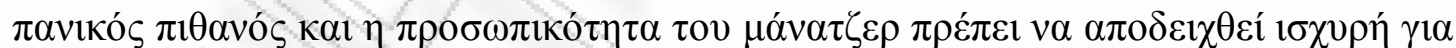

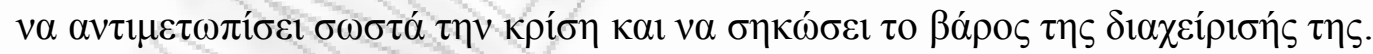

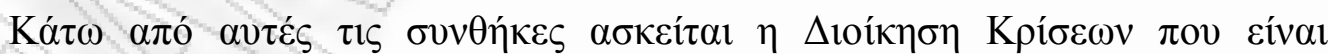

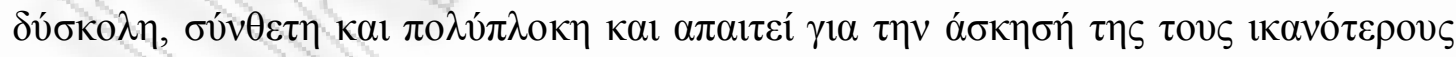

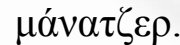

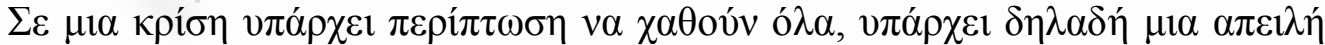

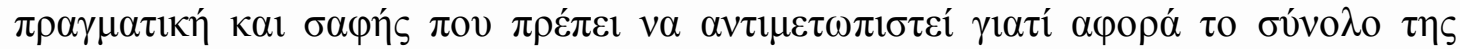

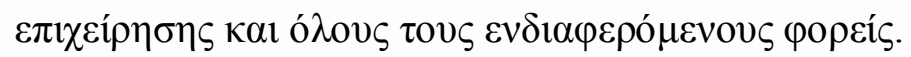




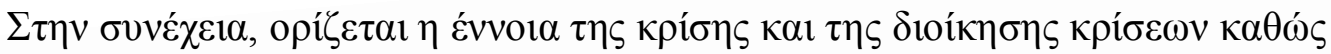

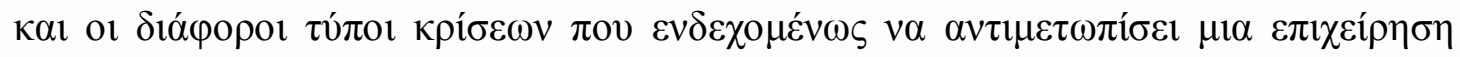
o $\pi 010 v \delta \eta ́ \pi 0 \tau \varepsilon \kappa \lambda \alpha ́ \delta o v$.

\section{КЕФАААIO 2}

\subsection{Opıбнoí}

\subsubsection{Kрí́ø}

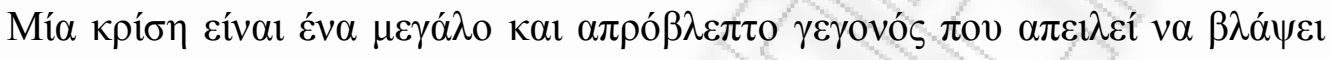

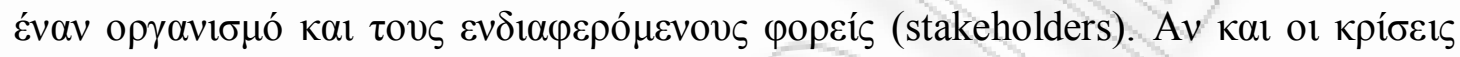

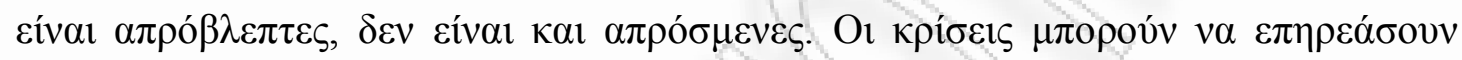

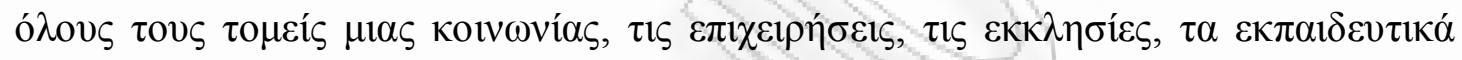

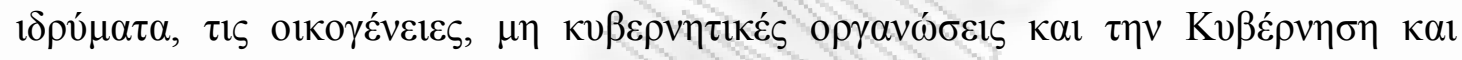

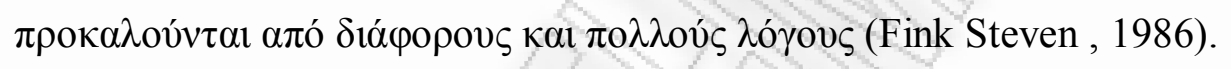

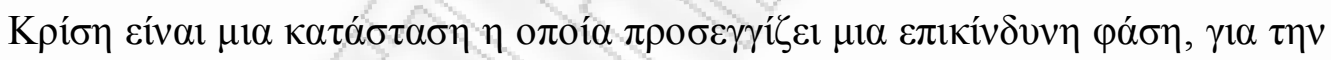

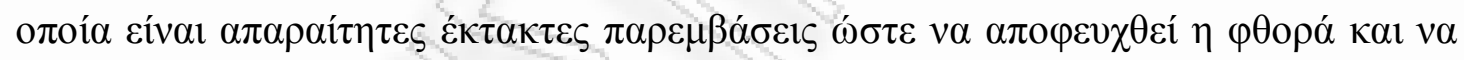

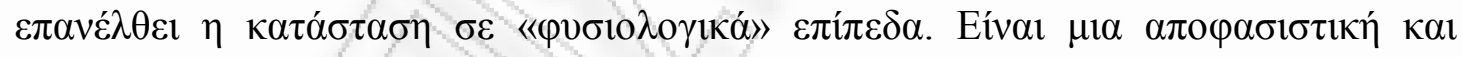

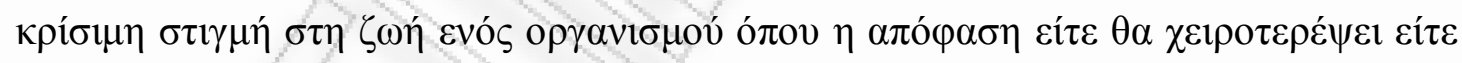
$\theta \alpha \kappa \alpha \lambda v \tau \varepsilon \rho \varepsilon ́ \psi \varepsilon \iota \tau \eta \nu ~ \kappa \alpha \tau \alpha ́ \sigma \tau \alpha \sigma \eta$ (R.Kanter , 1996).

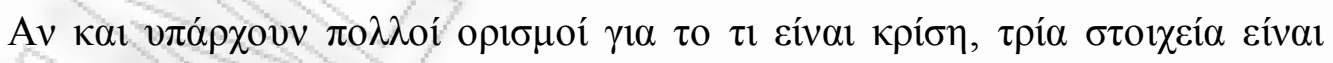

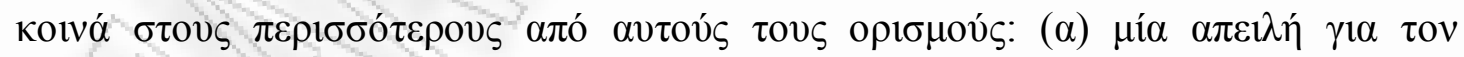

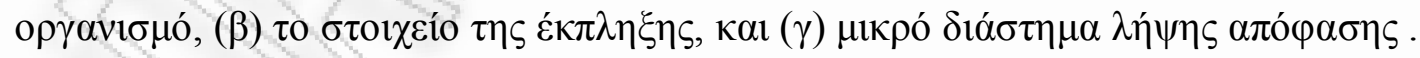

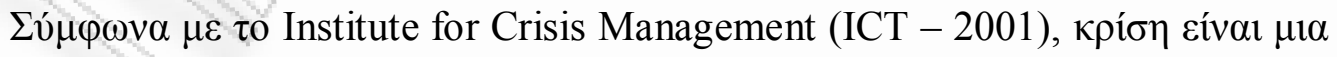

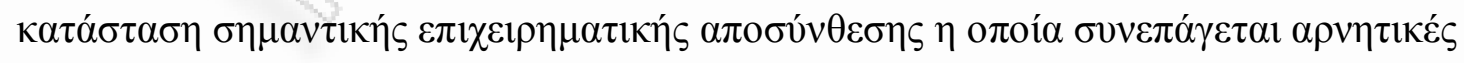

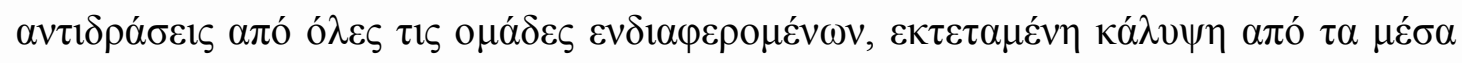

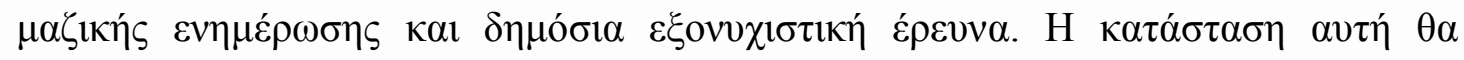




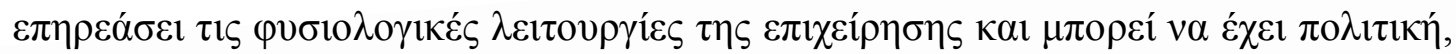

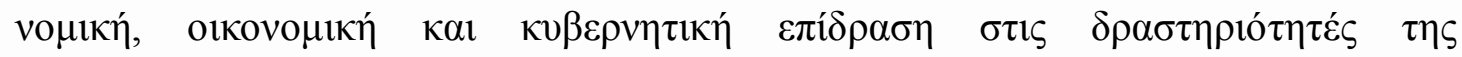
( www.crisisexperts.com ).

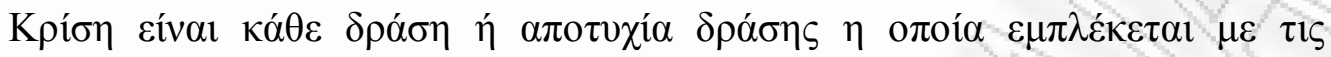

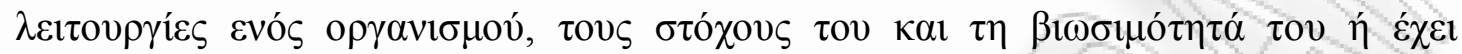

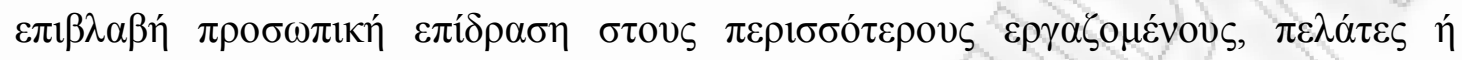

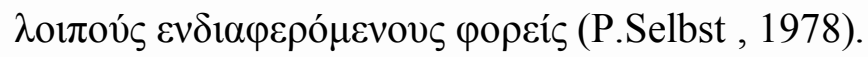

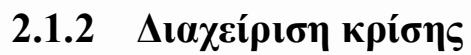

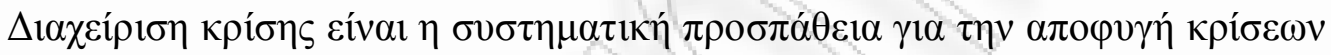

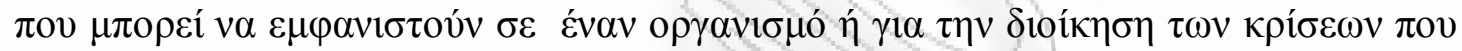

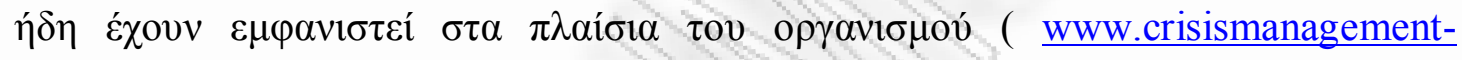
disasterrecovery.com )

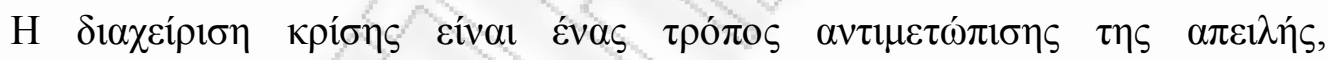

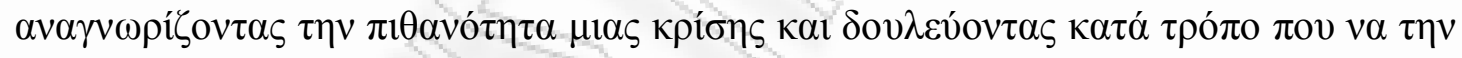

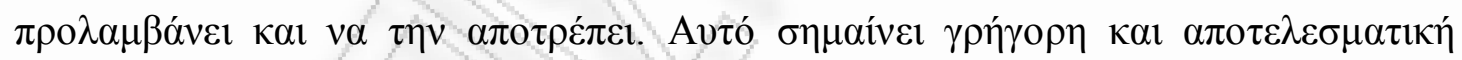

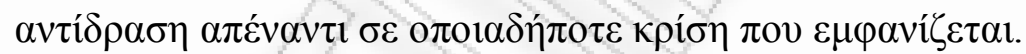

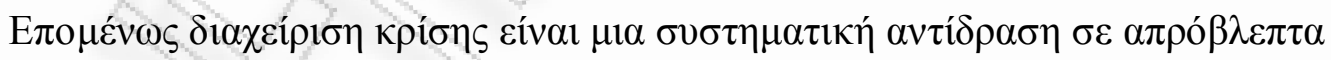

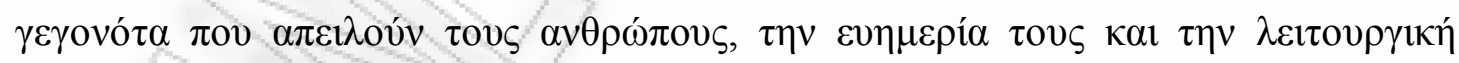

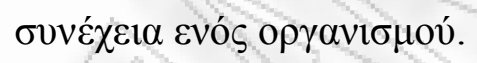

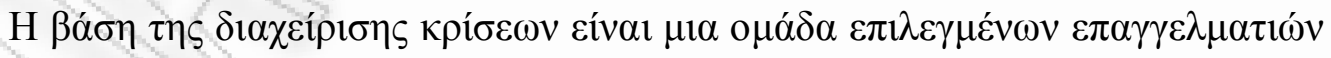

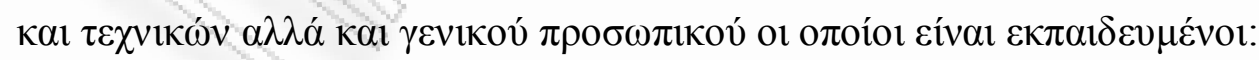

- $\sigma \tau \eta v \alpha v \alpha ́ \lambda v \sigma \eta \kappa \alpha 1 \varepsilon \kappa \tau i ́ \mu \eta \sigma \eta \tau \omega v \alpha \pi \varepsilon 1 \lambda \omega ́ v$

- $\quad \sigma \tau \eta v ~ \beta \varepsilon \lambda \tau i ́ \omega \sigma \eta ~ \kappa \alpha \iota ~ \varepsilon \varphi \alpha \rho \mu о \gamma \eta ́ ~ \varepsilon v \alpha \lambda \lambda \alpha \kappa \tau \imath \kappa \omega ́ v ~ \alpha \nu \tau \alpha \pi о \kappa \rho i ́ \sigma \varepsilon \omega \nu$ 


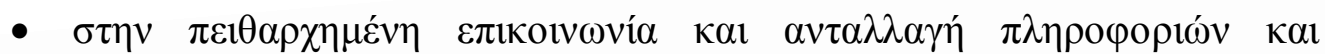
$\alpha \pi \circ \varphi \alpha ́ \sigma \varepsilon \omega v \kappa \alpha 1$

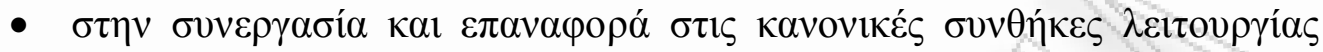
ó $\alpha \alpha \nu \eta \kappa \rho i ́ \sigma \eta ~ \varepsilon ́ \chi \varepsilon 1 ~ \varepsilon \pi \varepsilon ́ \lambda \theta \varepsilon 1$.

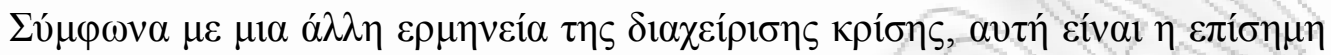

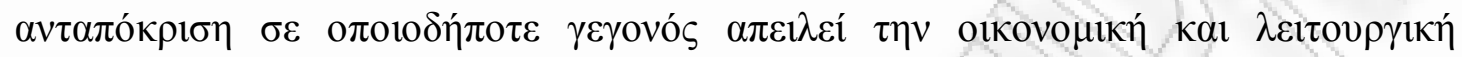

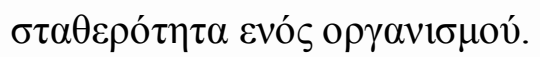

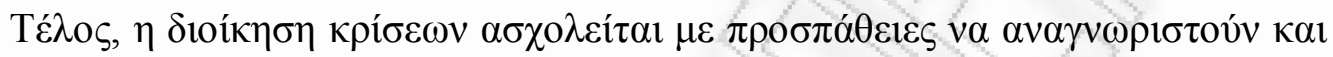

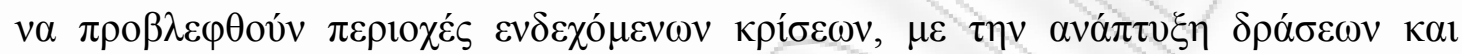

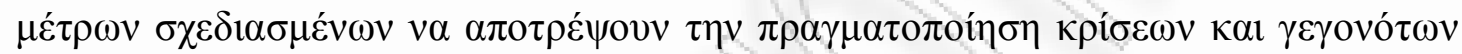

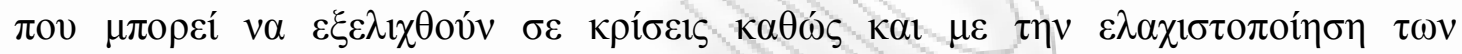

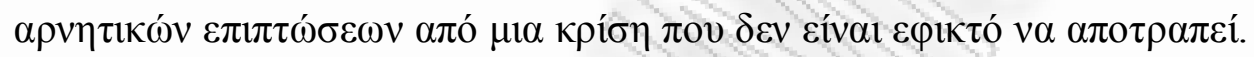

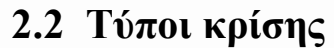

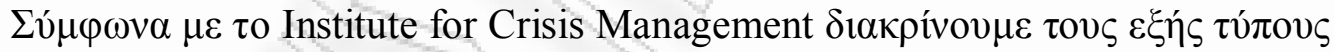

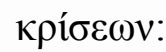

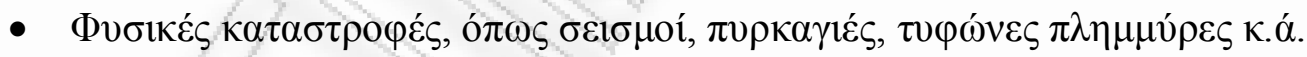

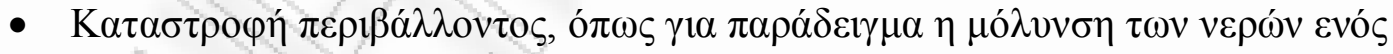

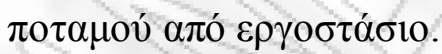

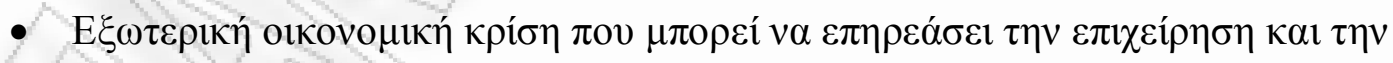

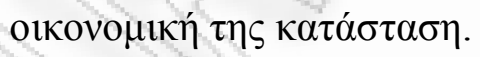

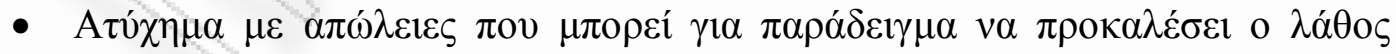

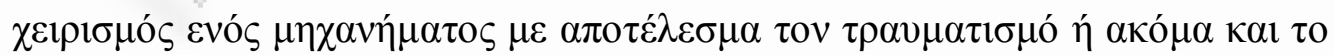

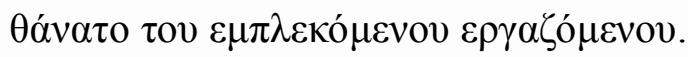




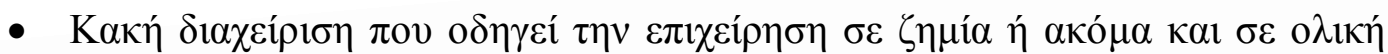
$\kappa \alpha \tau \alpha \sigma \tau \rho о \varphi \eta ́$.

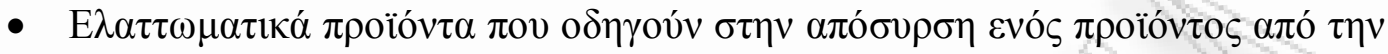
aүopá.

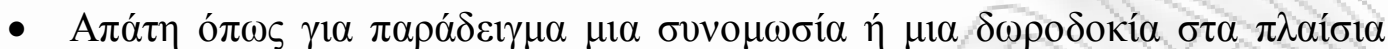

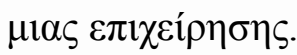

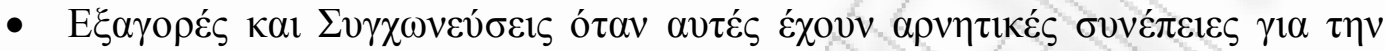

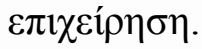

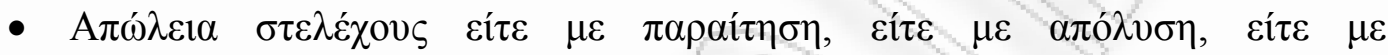

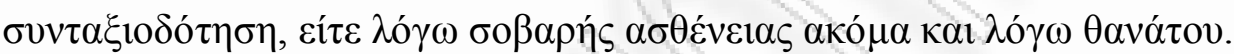

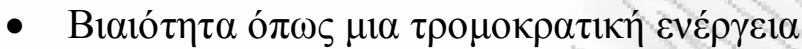

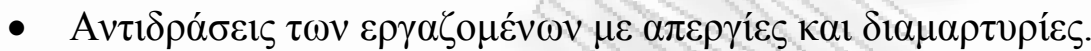

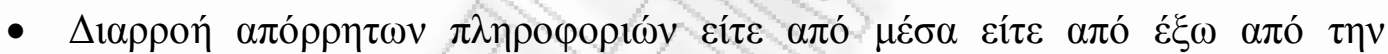

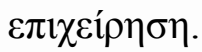

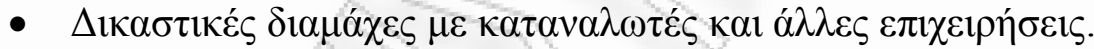

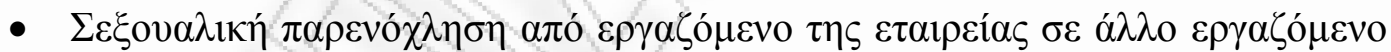
$\eta \dot{\eta} \pi \varepsilon \lambda \dot{\alpha} \tau \eta$

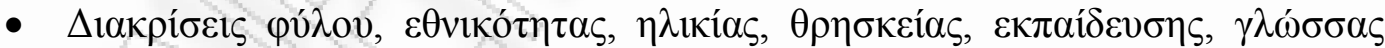
$\kappa . \alpha ́$. 


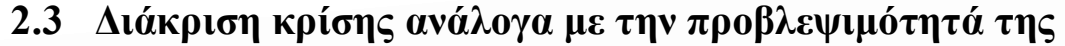

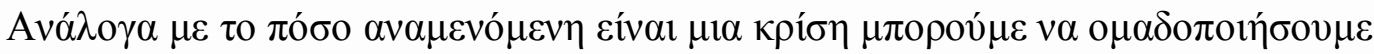

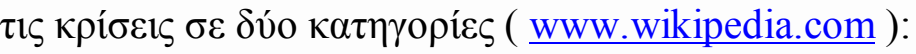

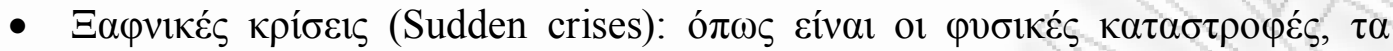

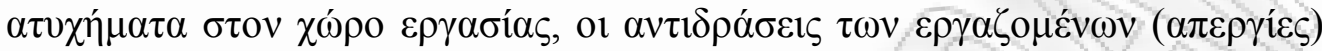

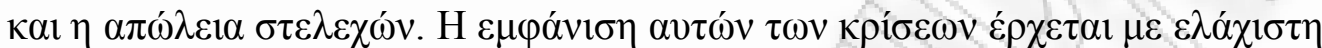

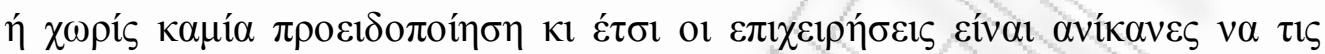
$\pi \rho \circ \beta \lambda \varepsilon ́ \psi o v v$.

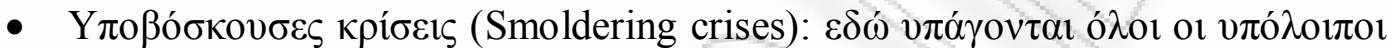

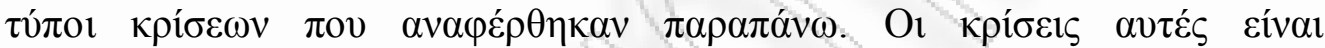
$\pi \varepsilon \rho 1 \sigma \sigma o ́ \tau \varepsilon \rho о ~ \pi \rho о \beta \lambda \varepsilon ́ \psi \psi \mu \varepsilon \varsigma$.

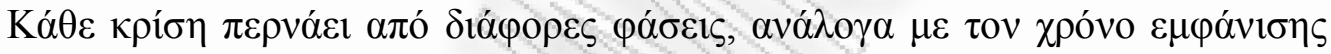

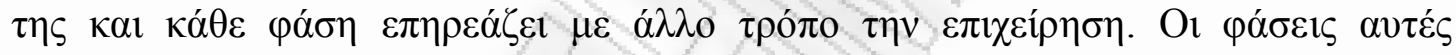

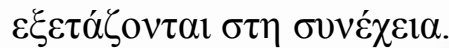

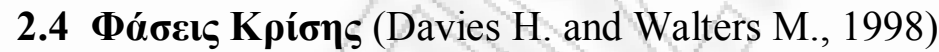

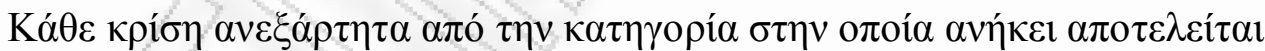

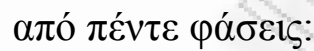

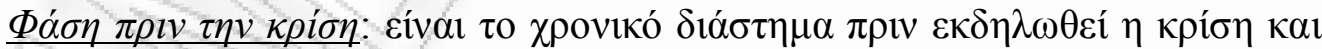

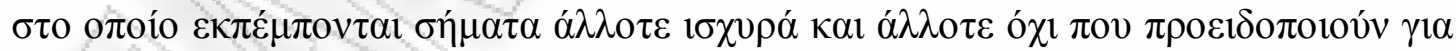

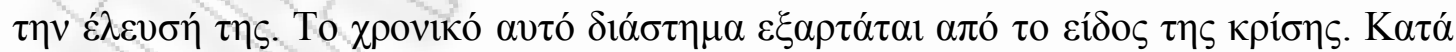

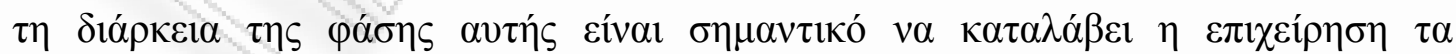

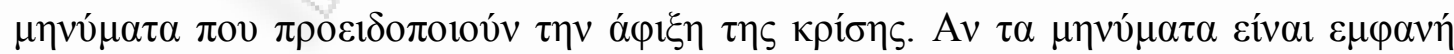

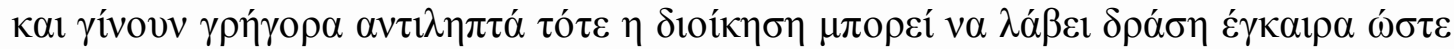

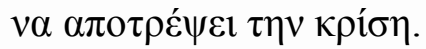




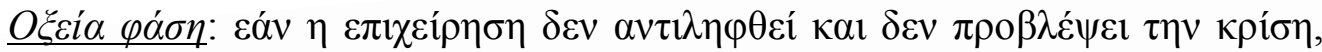

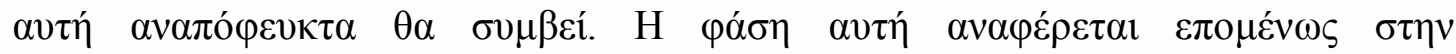

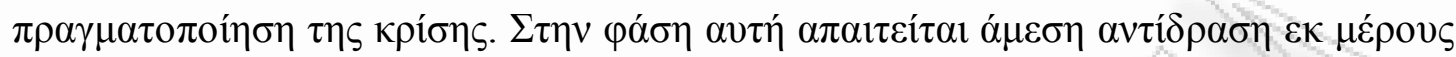

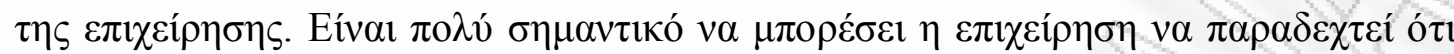

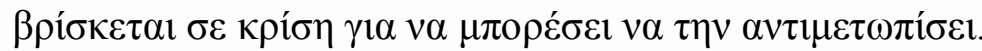

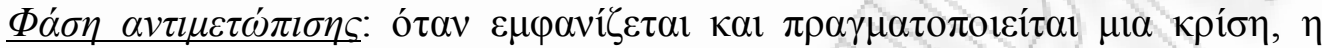

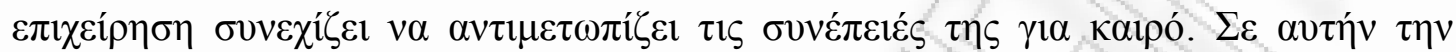

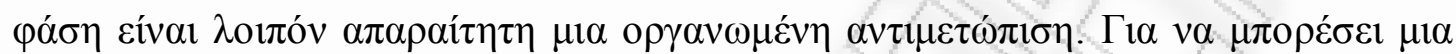

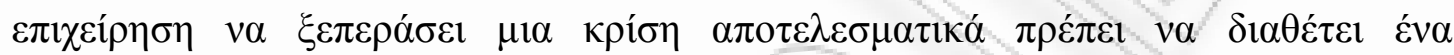

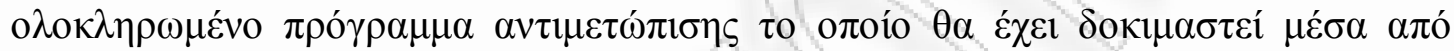

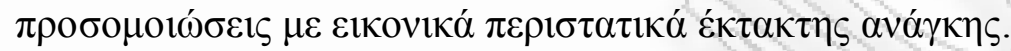

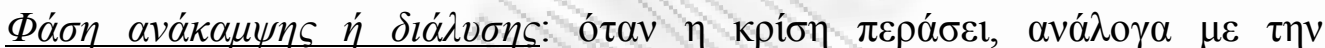

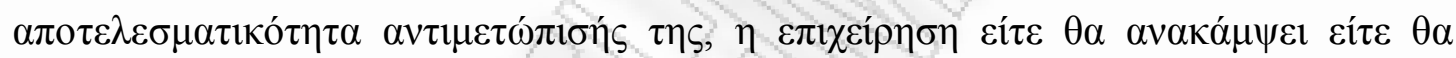
$\delta 1 \alpha \lambda v \theta \varepsilon i ́$.

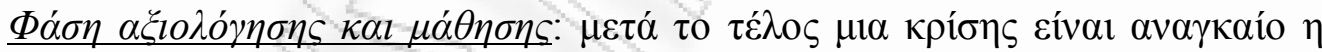

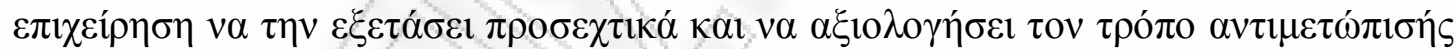

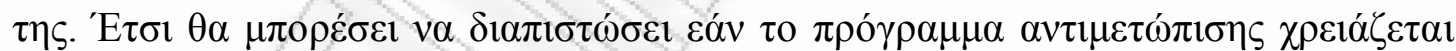

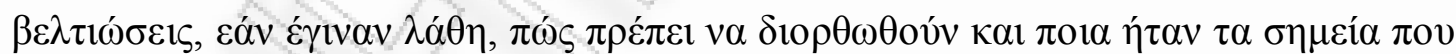

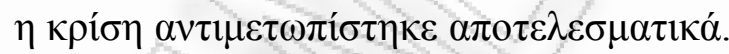

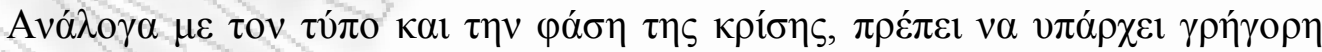

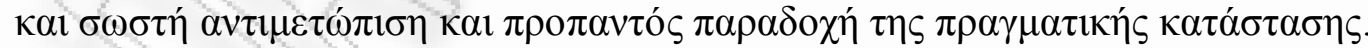




\section{КЕФАААIO 3}

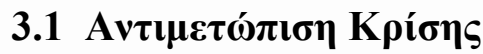

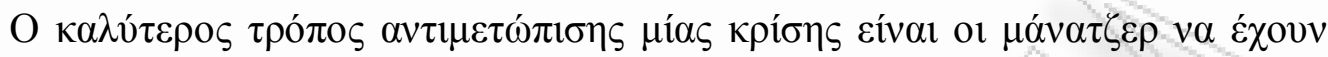

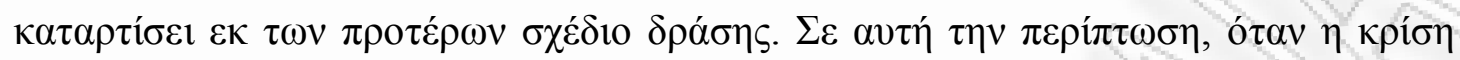

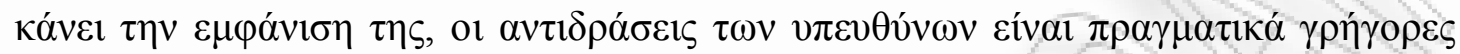

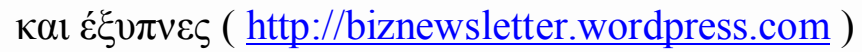

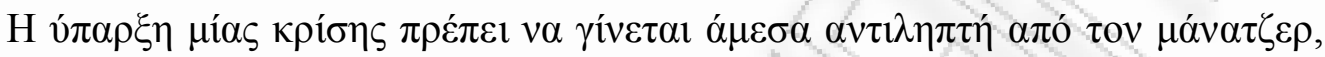

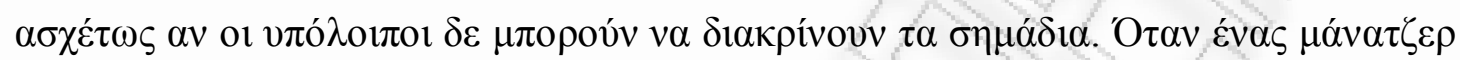

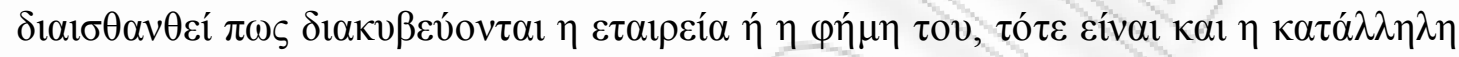

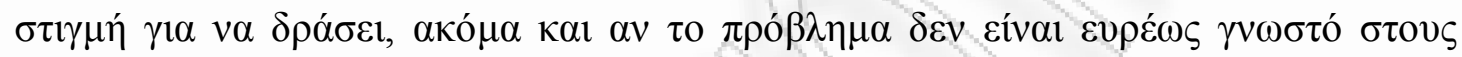

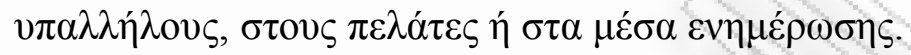

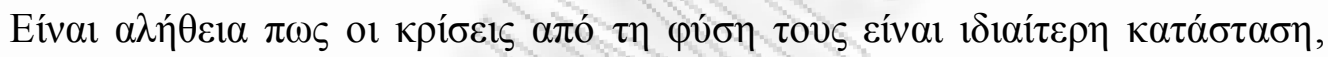

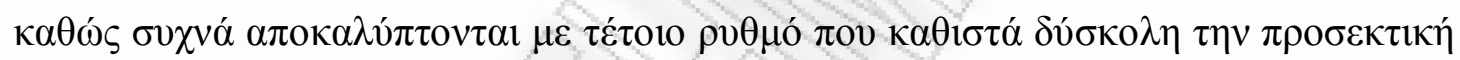

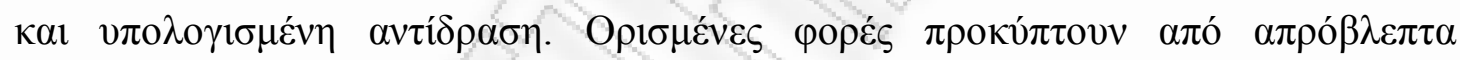

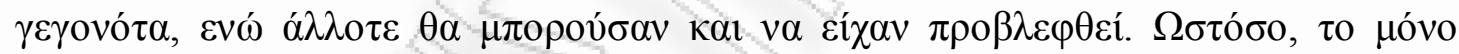

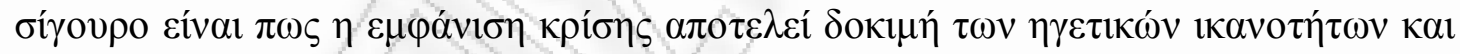

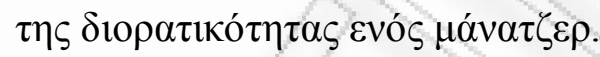

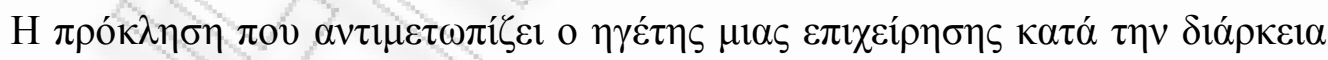

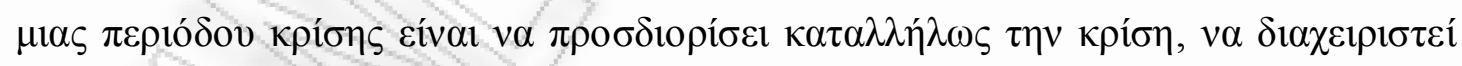

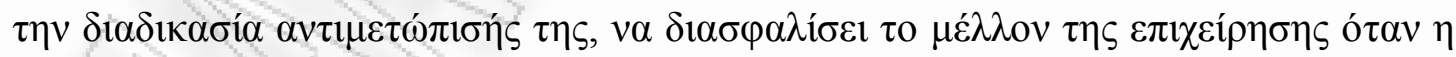

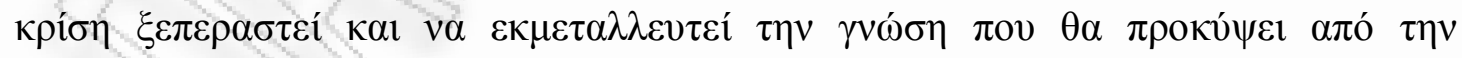

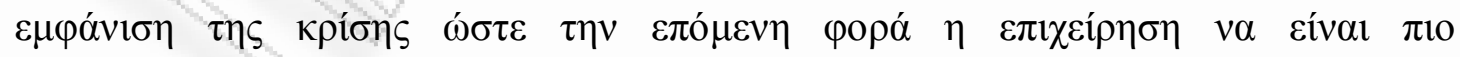

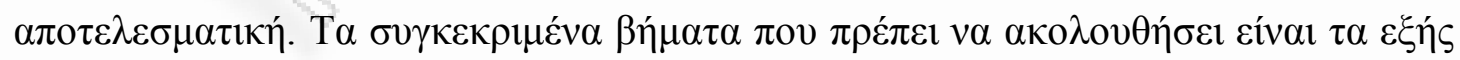
(R.Kanter, 1996): 


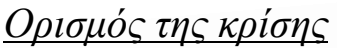

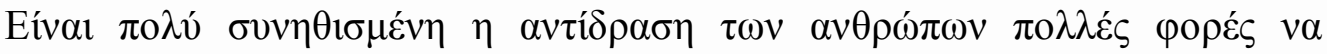

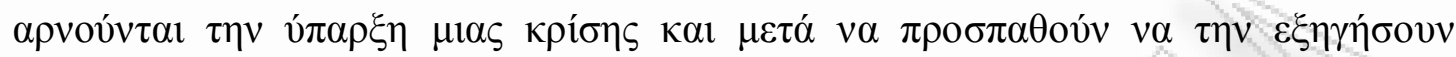

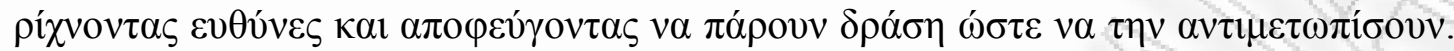

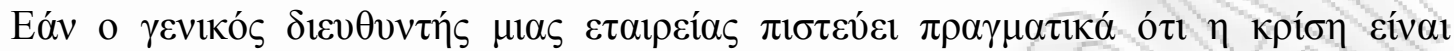

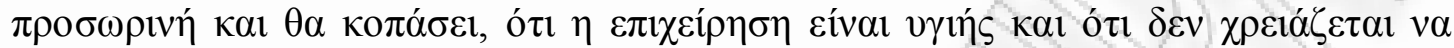

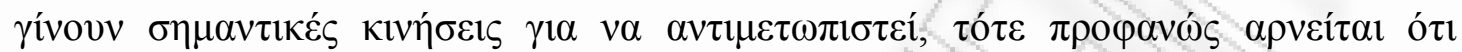

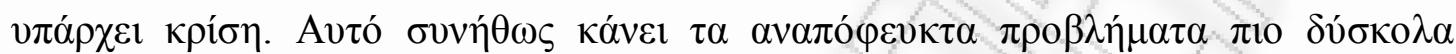

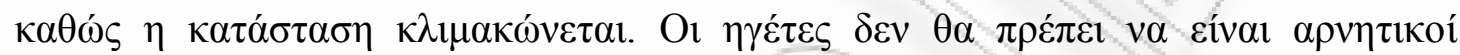

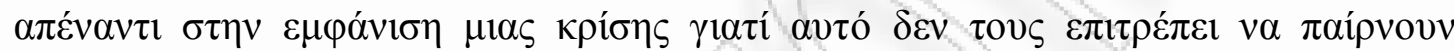
$\sigma \omega \sigma \tau \varepsilon ́ \varsigma \alpha \pi \circ \varphi \alpha ́ \sigma \varepsilon 1 \zeta$.

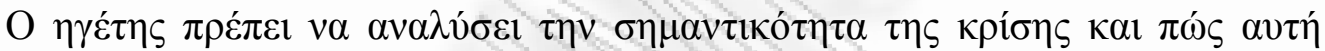

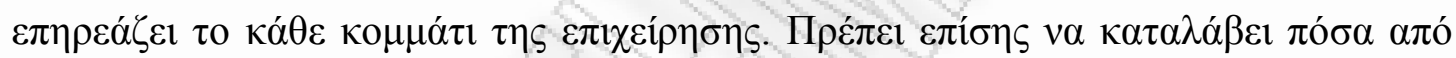

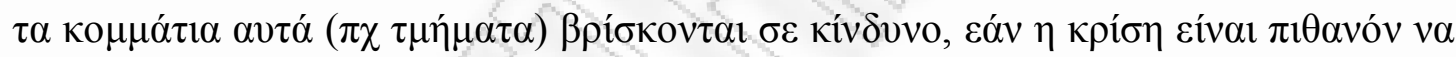

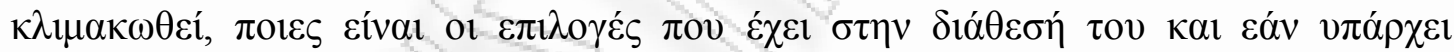

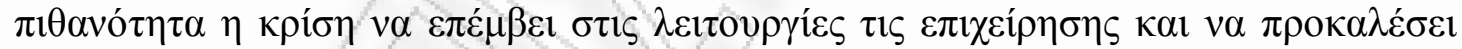
$\mu \varepsilon i ́ \omega \sigma \eta \tau \eta \varsigma \alpha v \tau o \pi \varepsilon \pi \circ i ́ \theta \eta \sigma \eta \varsigma$.

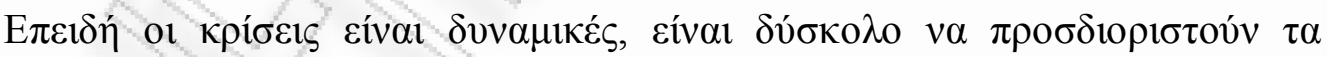

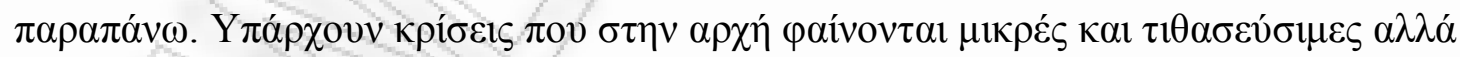

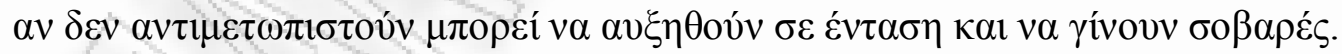

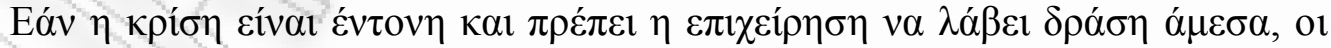

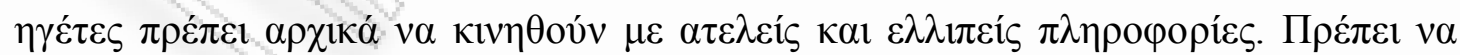

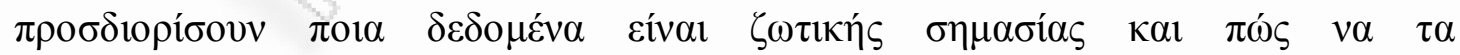

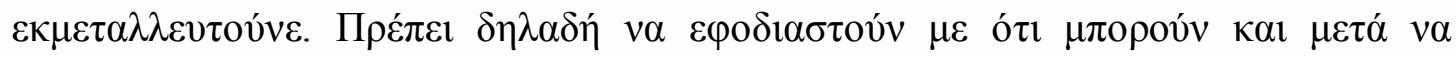

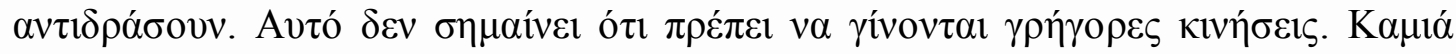




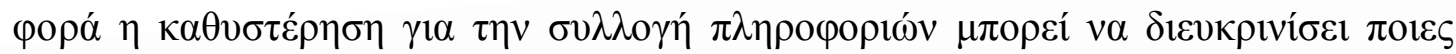

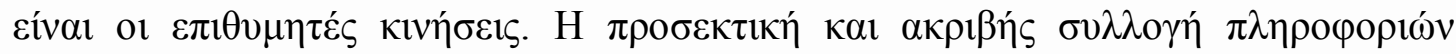

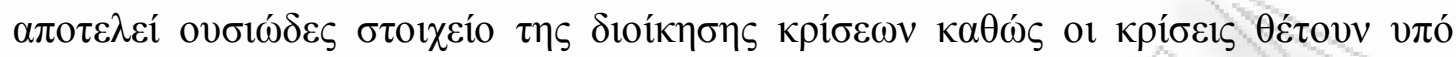

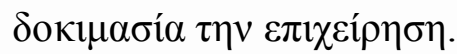

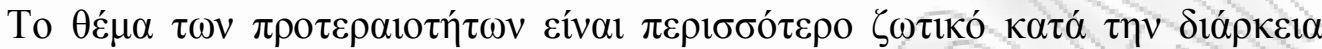

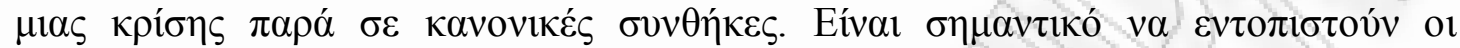

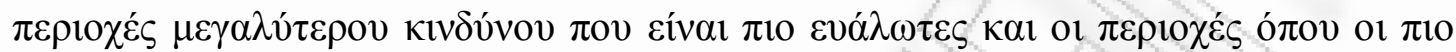

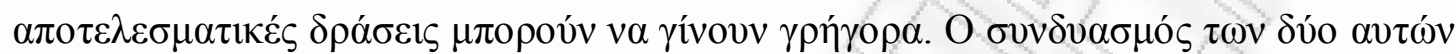

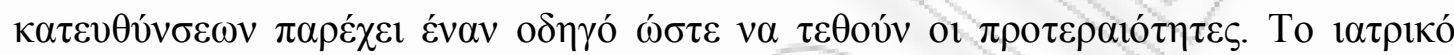

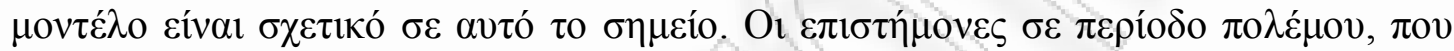

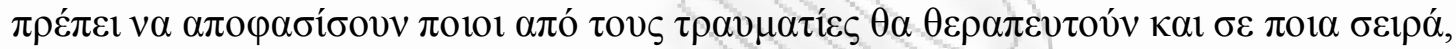

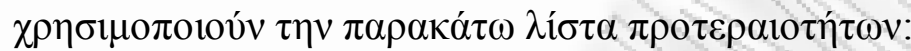

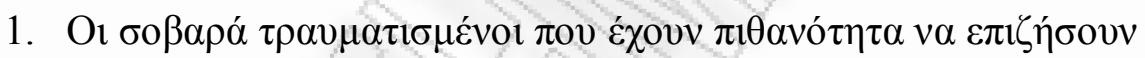

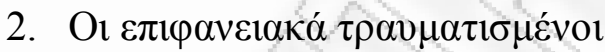

3. Oı $\alpha \delta 1 o ́ \rho \theta \omega \tau \alpha \tau \rho \alpha \nu \mu \alpha \tau 1 \sigma \mu \varepsilon ́ v o 1$

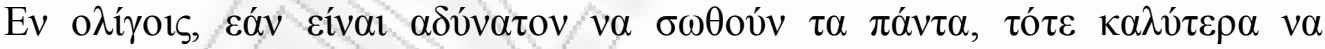

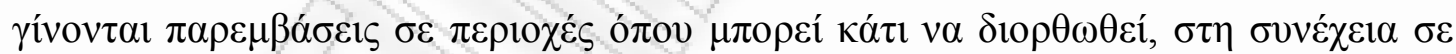

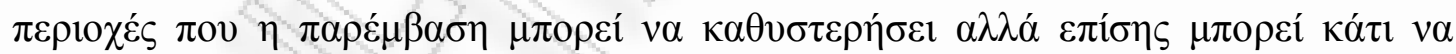

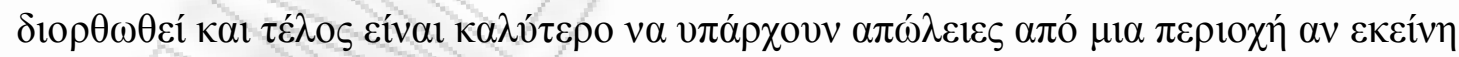

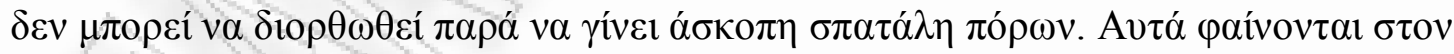
$\pi$ ív $\alpha \kappa \alpha 2$. 


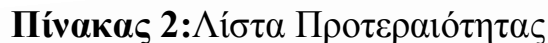

\begin{tabular}{|c|c|c|}
\hline $\begin{array}{l}\text { IEIPA } \\
\text { IDPOTEPAIOTHTAL }\end{array}$ & 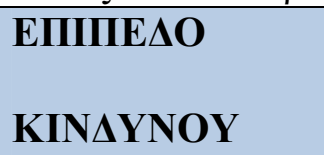 & $\begin{array}{l}\text { EN } \triangle E X O M E N H \\
\text { EПITYXIA }\end{array}$ \\
\hline ПЕРIOХН $1^{\mathrm{H}}$ & 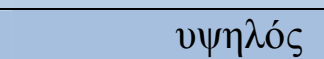 & 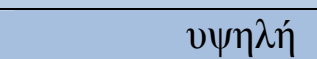 \\
\hline ПЕРIOХН $2^{\mathrm{H}}$ & $\chi \alpha \mu \eta \lambda o^{\prime} \varsigma$ & $v \psi \eta \lambda \eta ́$ \\
\hline ПЕРІОХН $3^{\mathrm{H}}$ & 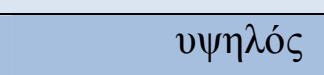 & $\chi \alpha \mu \eta \lambda \eta ́$ \\
\hline
\end{tabular}

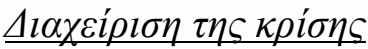

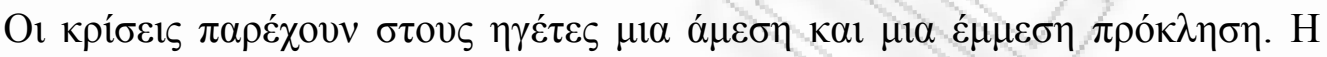

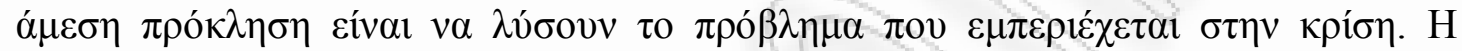

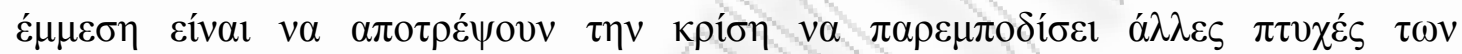

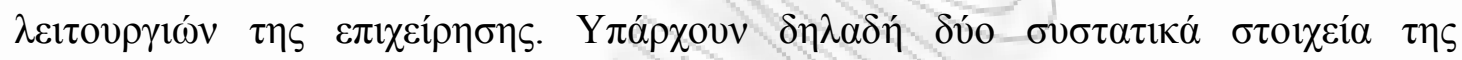

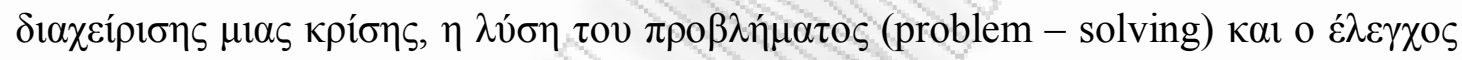

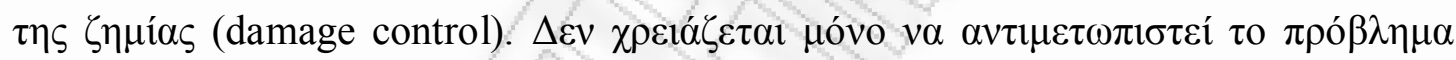

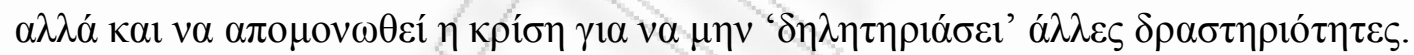

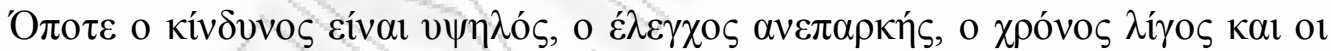

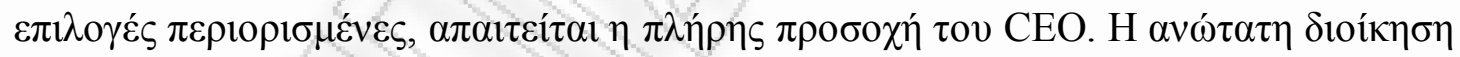

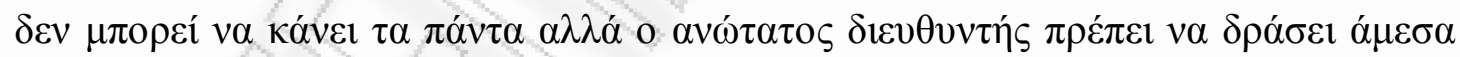

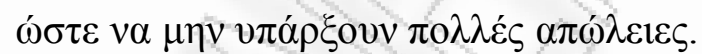

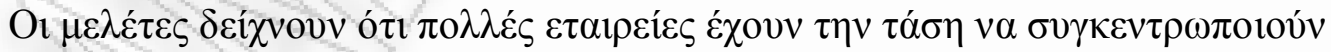

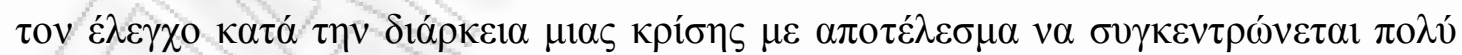

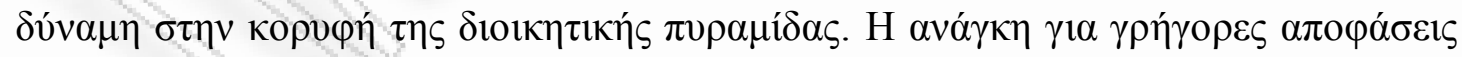

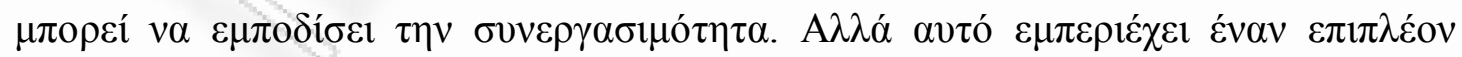

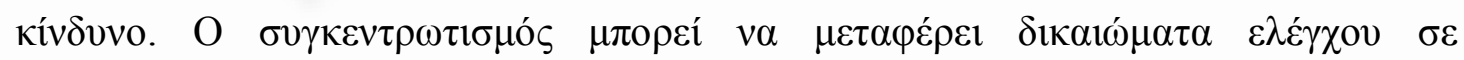

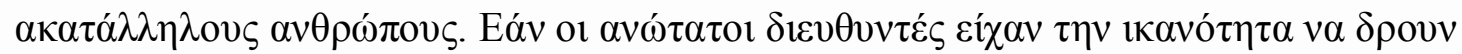

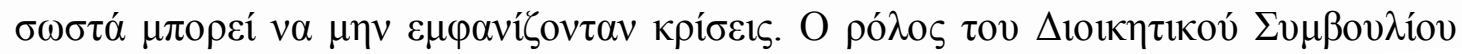




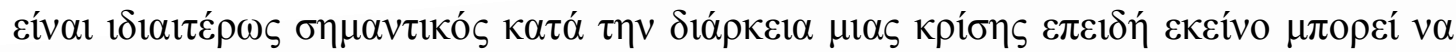

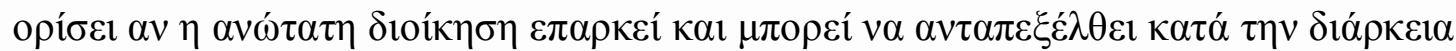

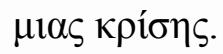

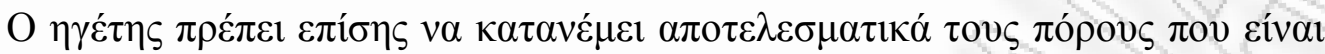

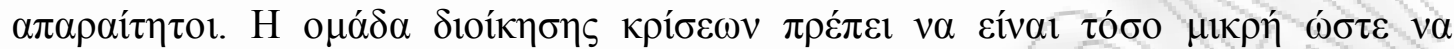

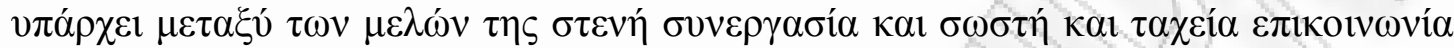

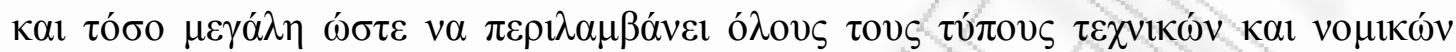

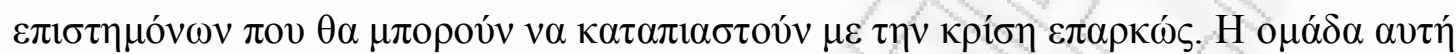

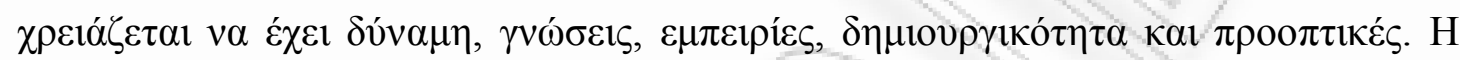

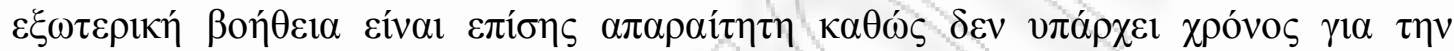

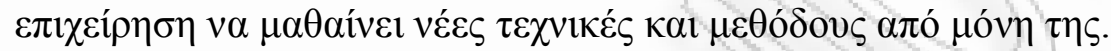

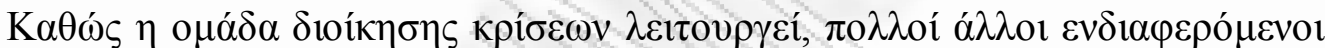

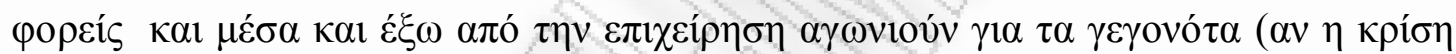

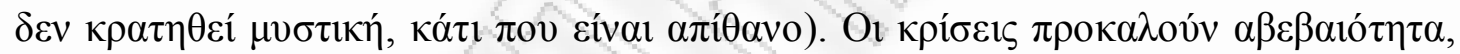

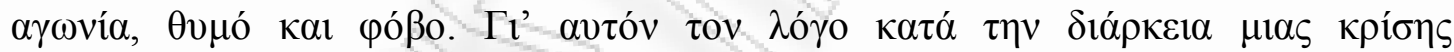

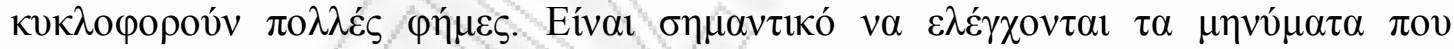

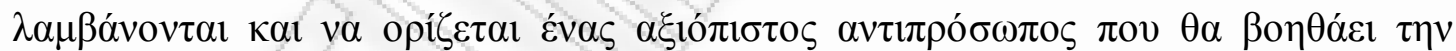

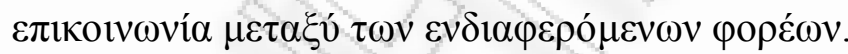

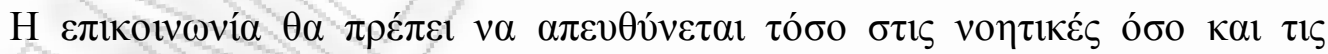

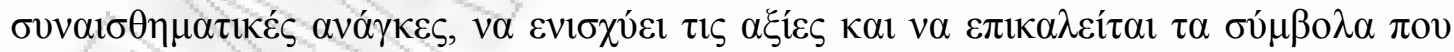

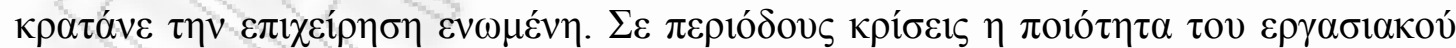

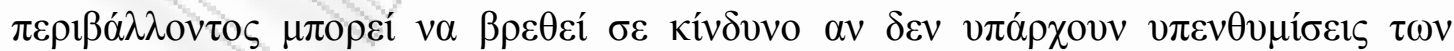

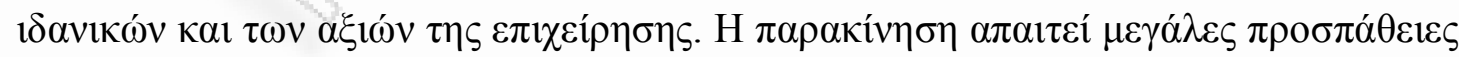

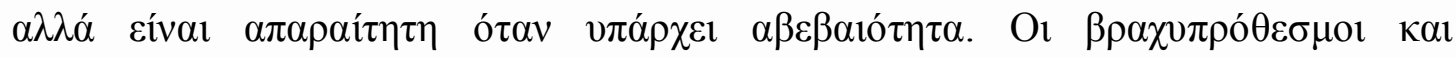




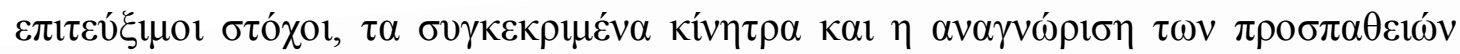

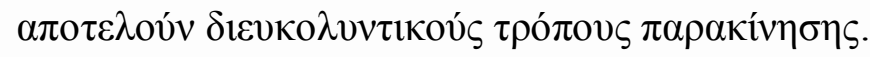

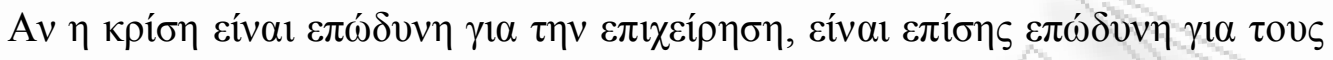

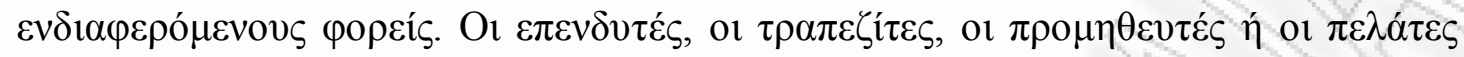

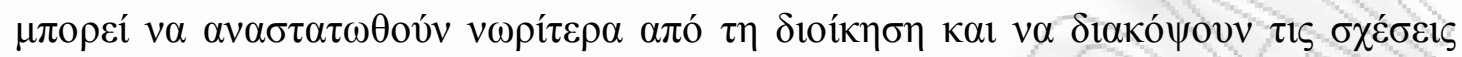

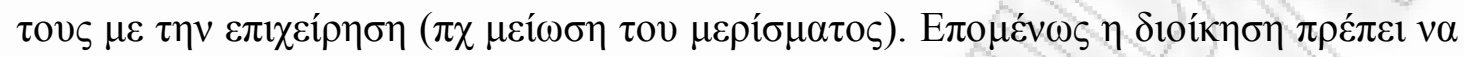

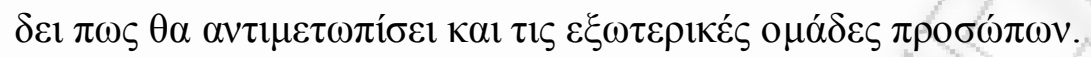

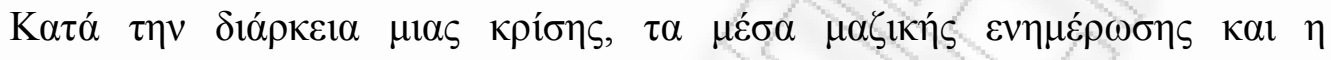

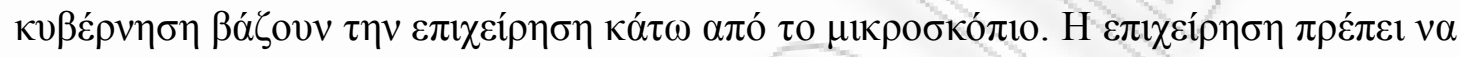

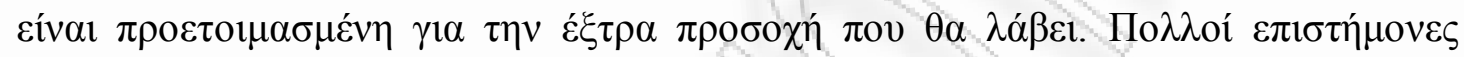

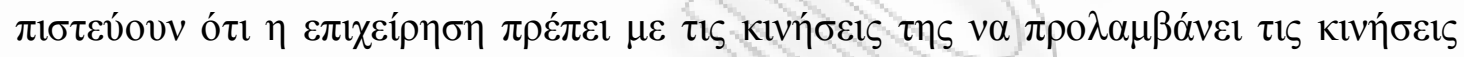
$\kappa \alpha \imath \tau \alpha$ $\chi 0 ́ \lambda \imath \alpha \tau \omega v$ MME.

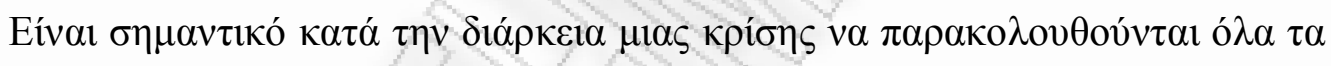

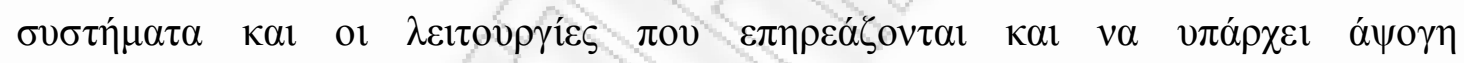

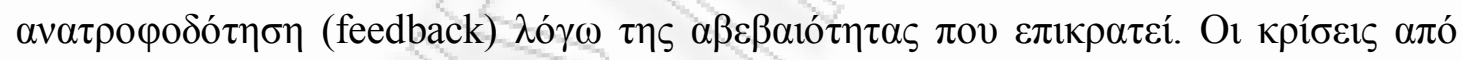

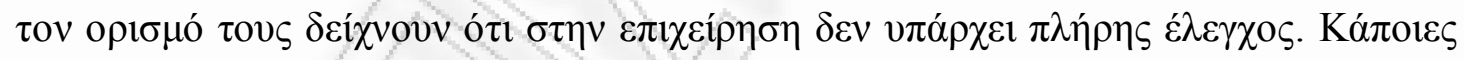

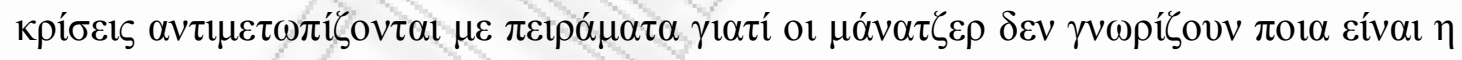

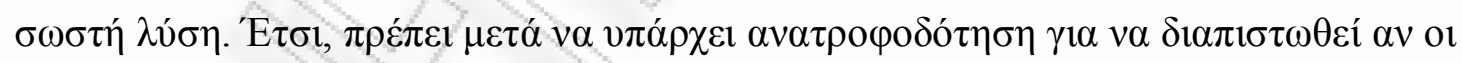

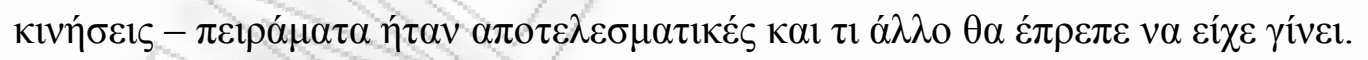

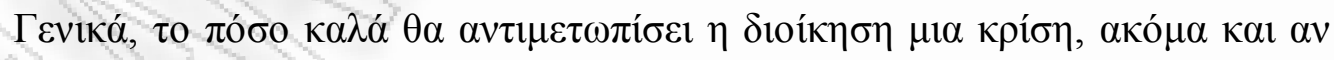

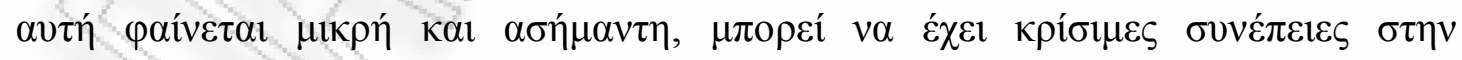

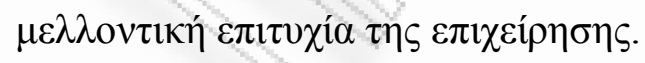

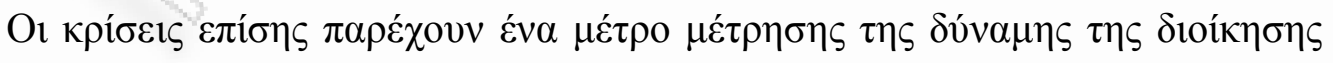

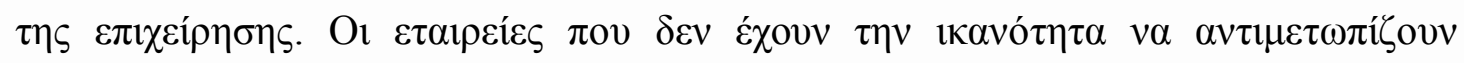

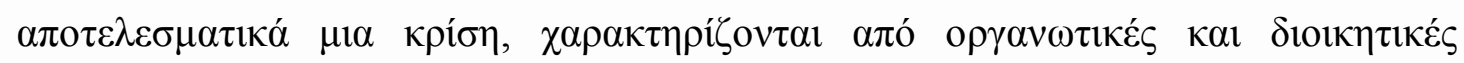




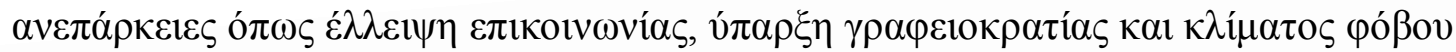

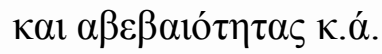

\section{$\underline{X \tau i \sigma l \mu o ~ \tau o v \mu \varepsilon^{\prime} \lambda \lambda o v \tau o \varsigma}$}

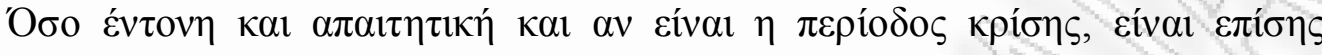

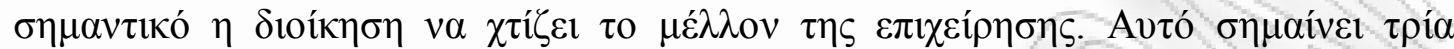
$\pi \rho \alpha ́ \gamma \mu \alpha \tau \alpha$

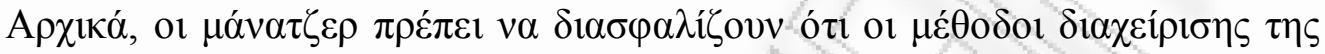

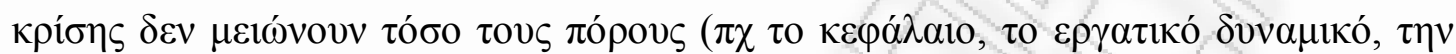

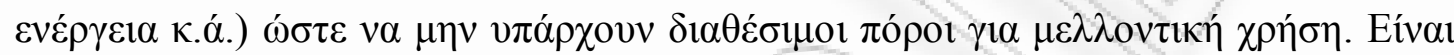

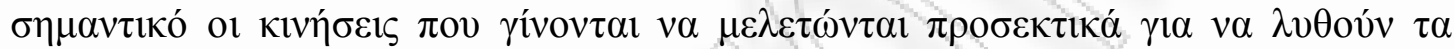

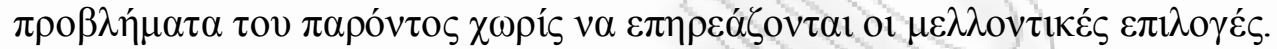

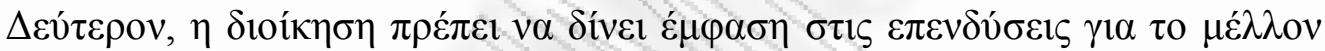

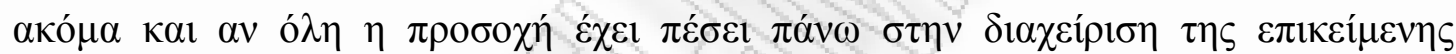

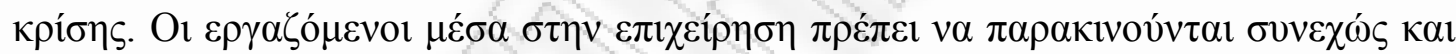

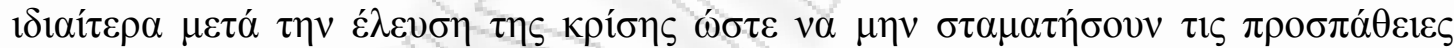

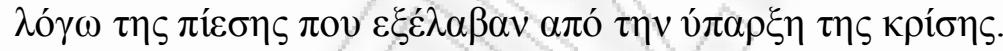

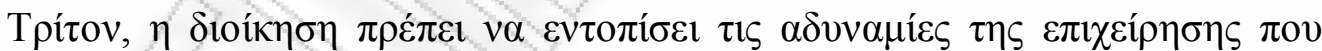

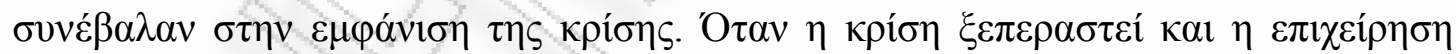

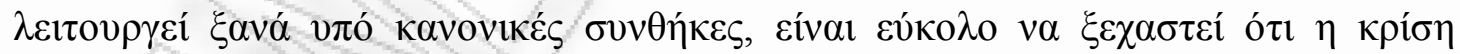

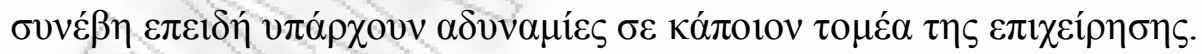

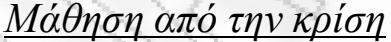

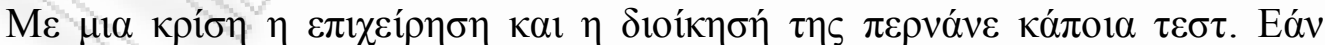

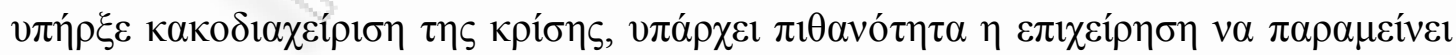

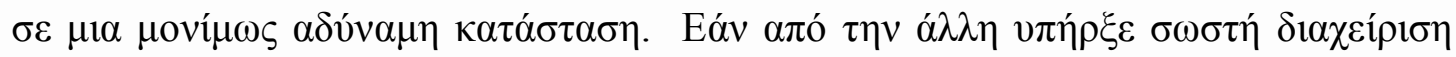

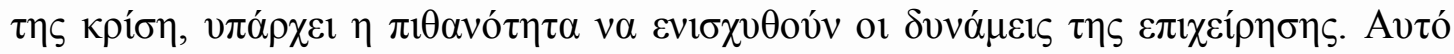




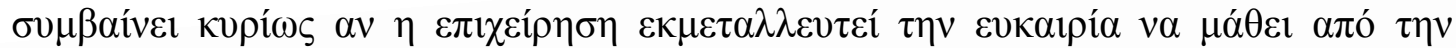

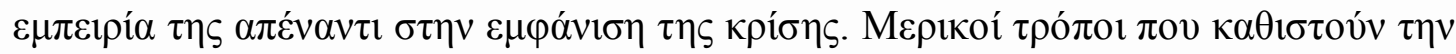

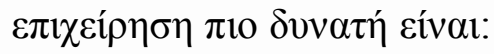

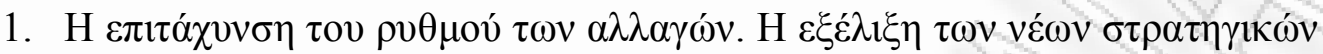

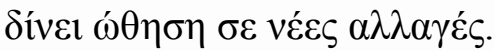

2. H $\alpha v \alpha \gamma v \omega ́ \rho ı\rceil \eta ~ \kappa \alpha \imath ~ \alpha v \tau \imath \mu \varepsilon \tau \omega ́ \pi \imath \sigma \eta \tau \omega v \alpha v \varepsilon ́ \kappa \delta \eta \lambda \omega v \pi \rho \circ \beta \lambda \eta \mu \alpha ́ \tau \omega v$.

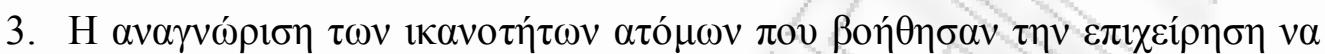

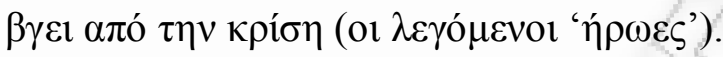

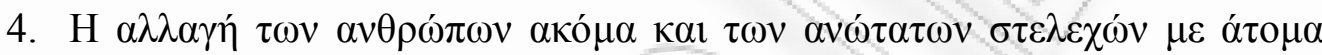

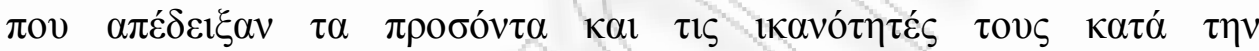

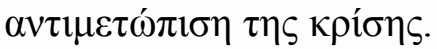

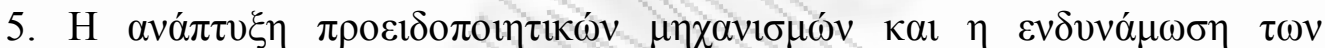

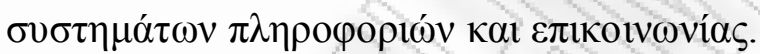

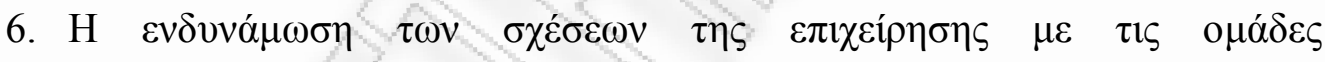
$\varepsilon v \delta 1 \alpha \varphi \varepsilon \rho \mu^{2} \nu \omega v$.

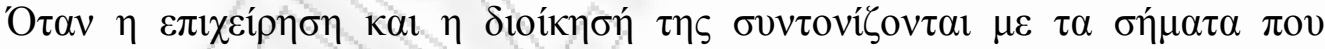

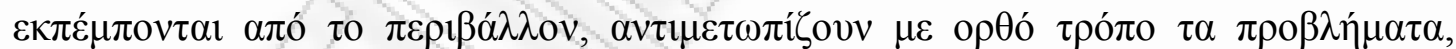

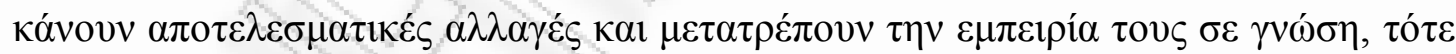

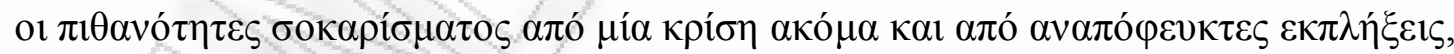

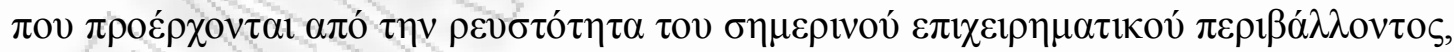
$\mu \varepsilon i \omega \vee \mathrm{ov} \tau \alpha 1$. 


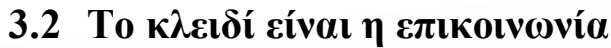

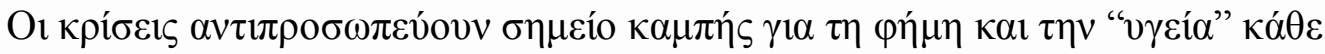

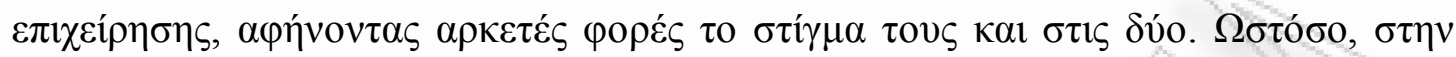

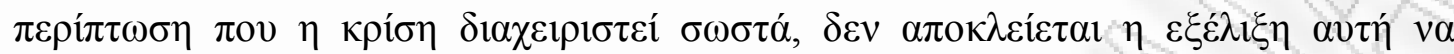

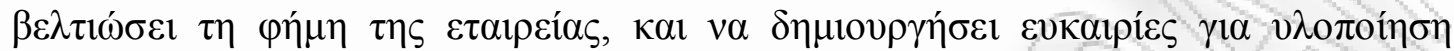

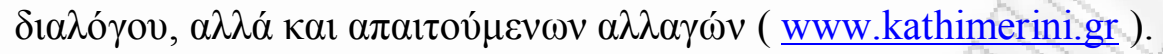

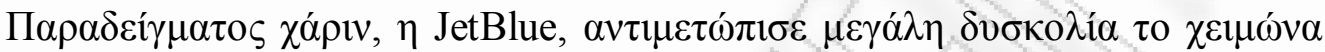

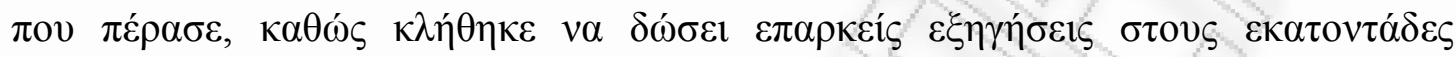

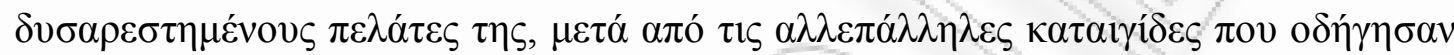

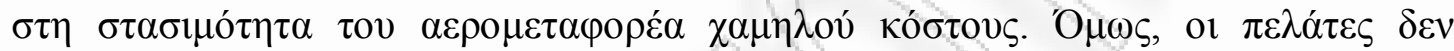

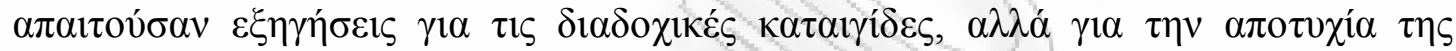

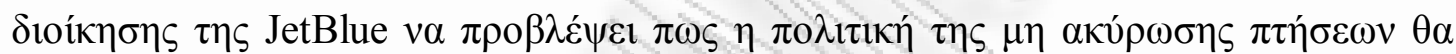

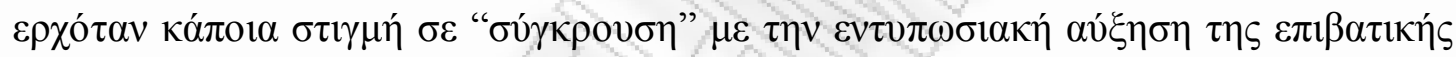

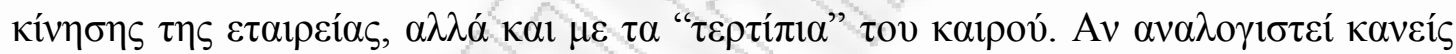

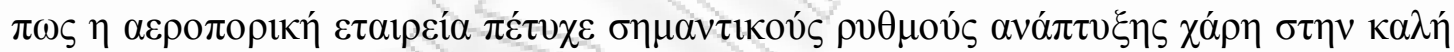

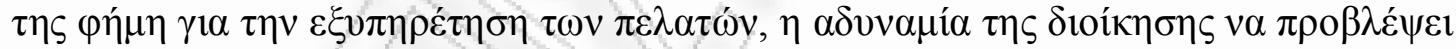

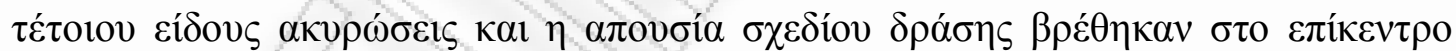

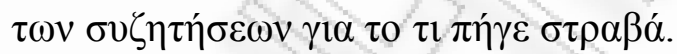

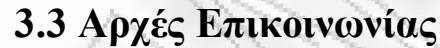

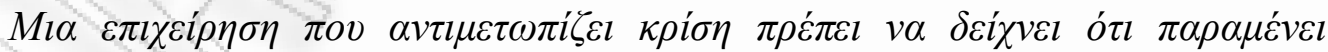

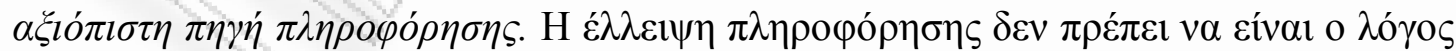

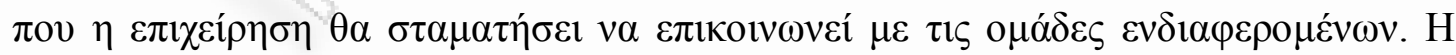

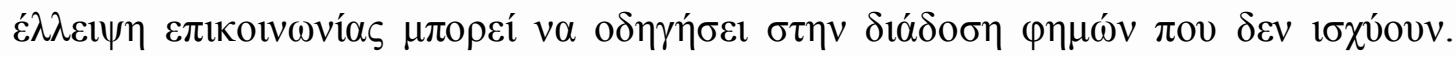

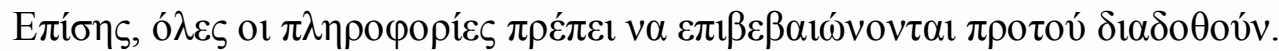




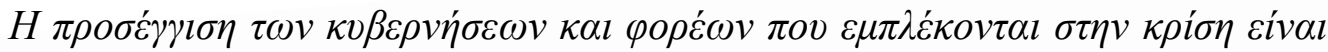

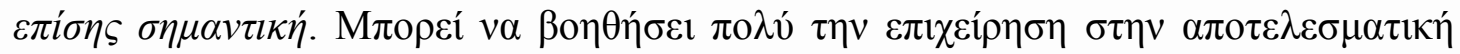

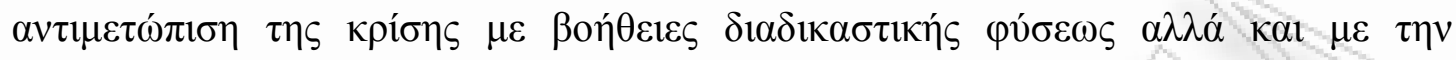

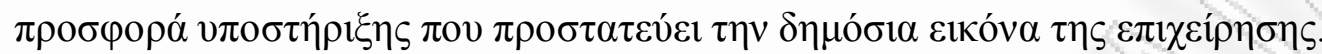

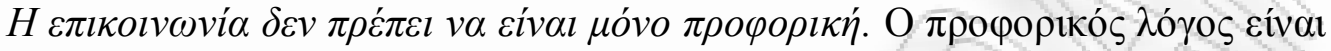

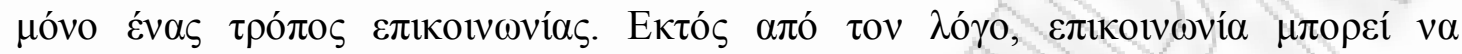

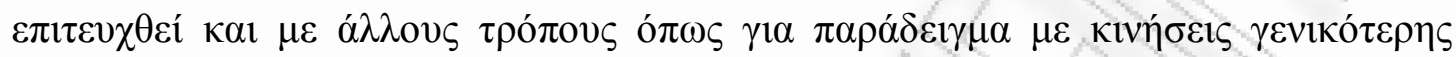

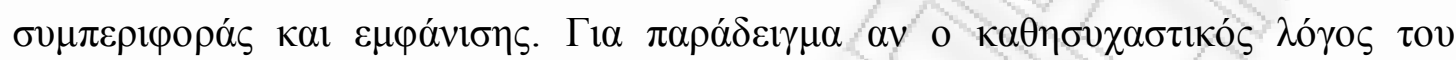

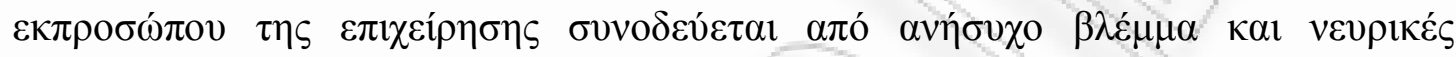

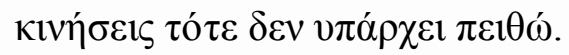

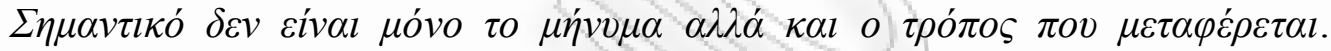

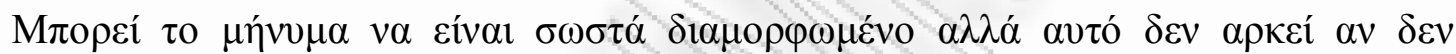

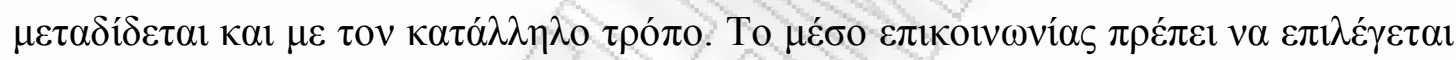

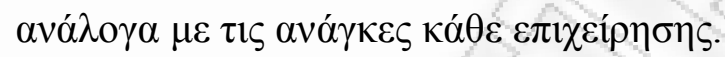

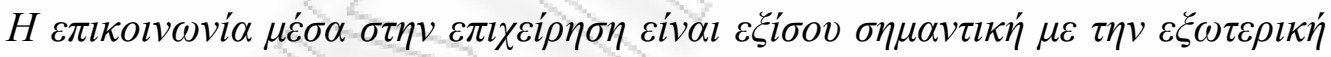

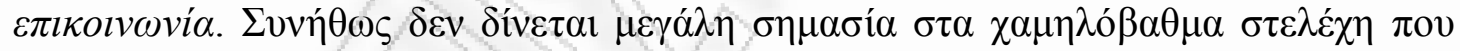

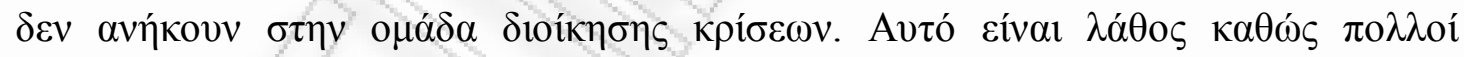

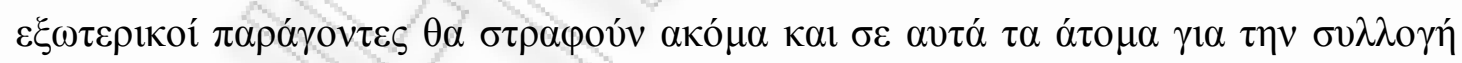

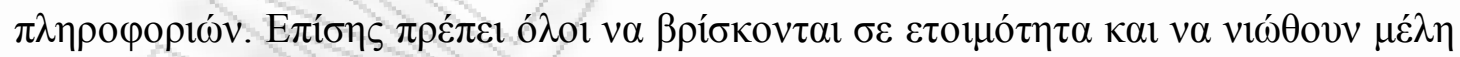

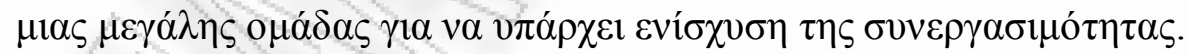




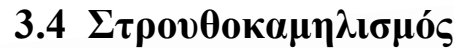

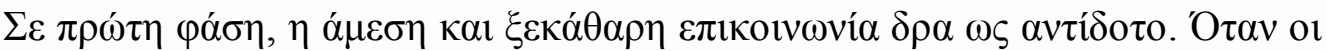

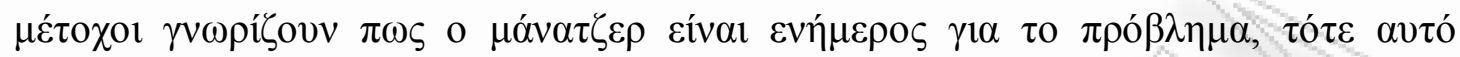

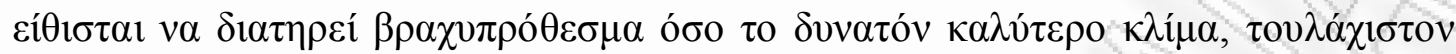

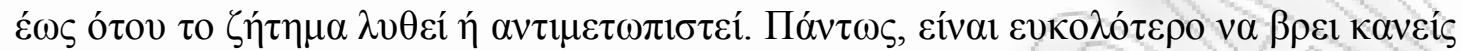

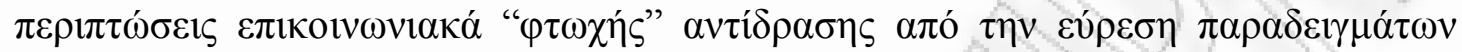

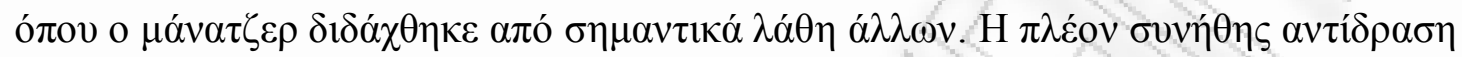

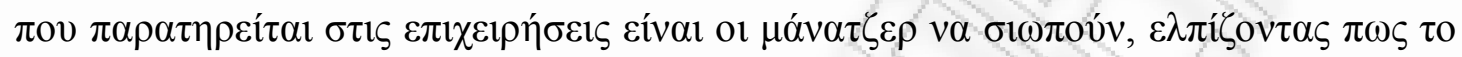

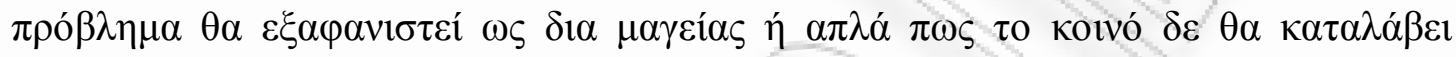

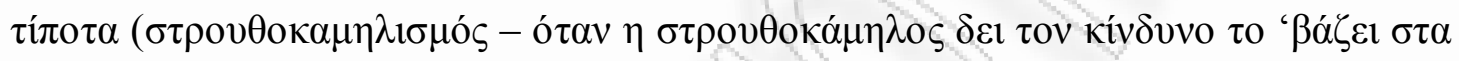

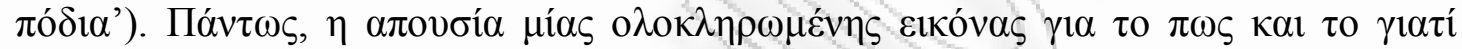

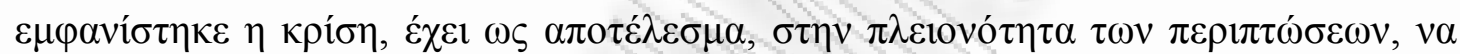

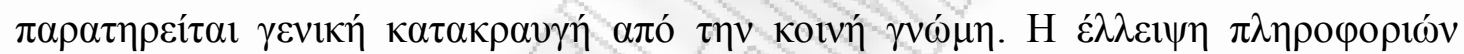

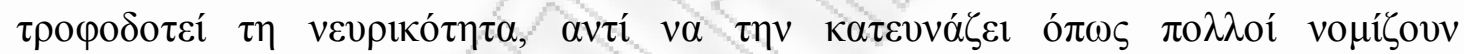
( www.israelnewsagency.com).

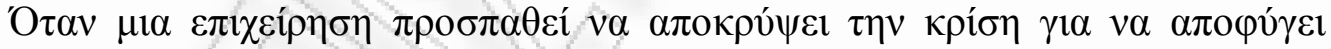

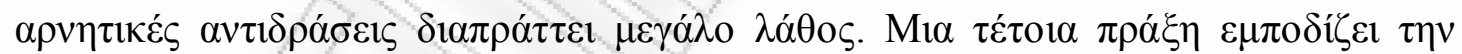

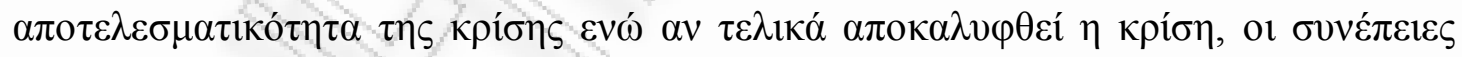

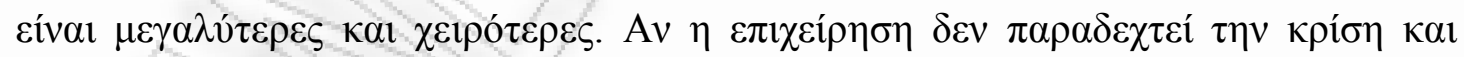

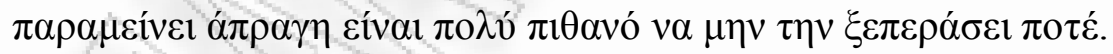

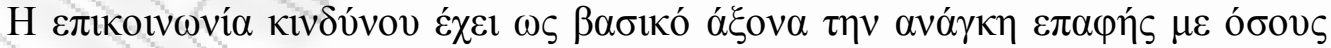

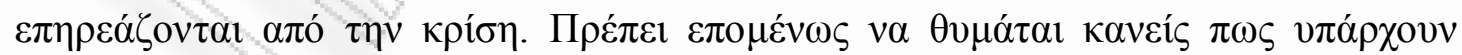

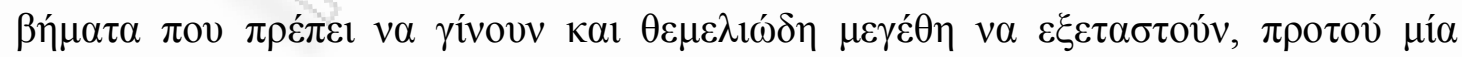

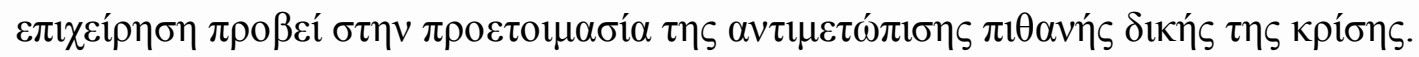




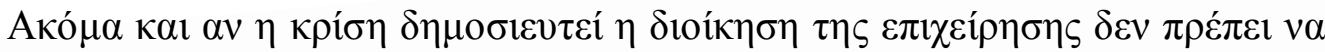

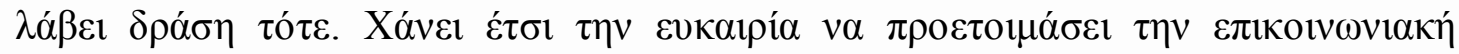

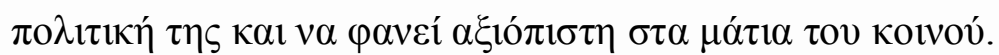

\section{КЕФАМАIO 4}

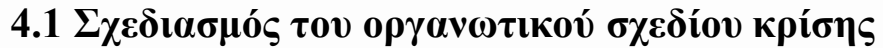

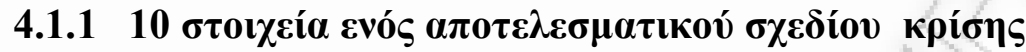

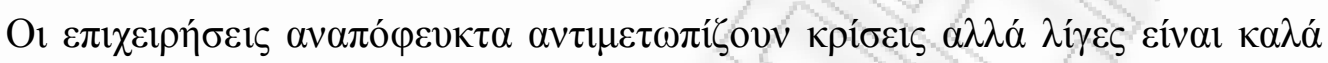

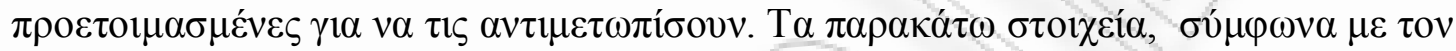

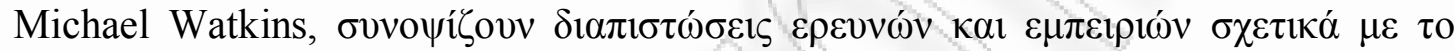

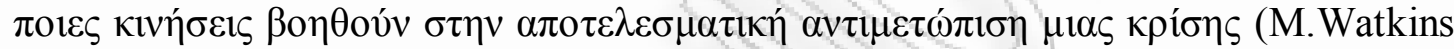
, 2001):

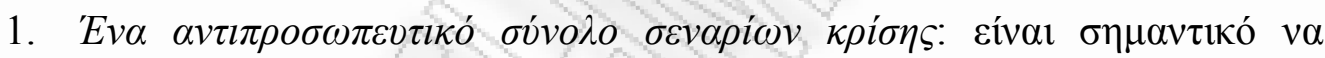

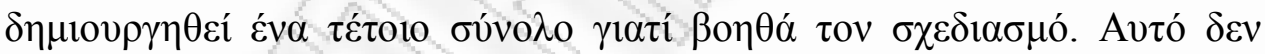

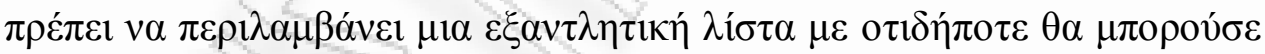

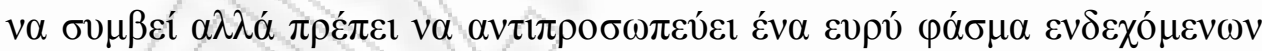

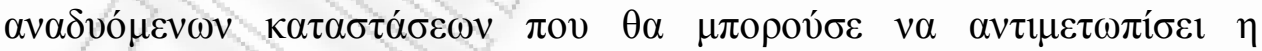

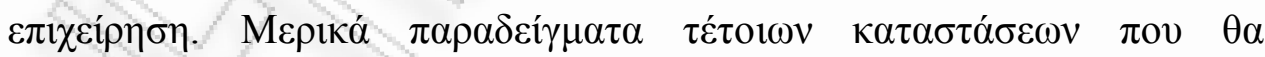

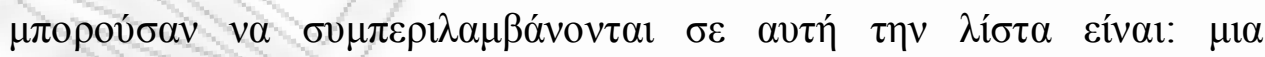

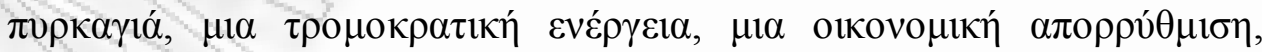

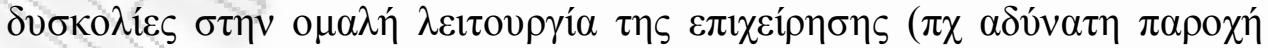

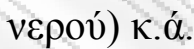

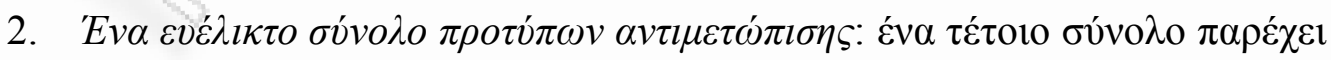

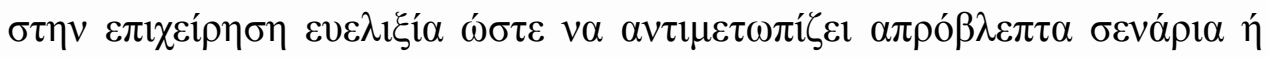

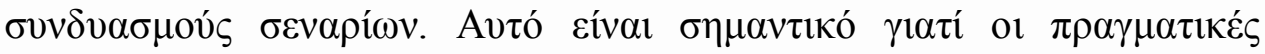




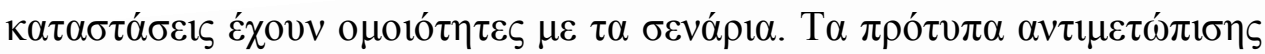

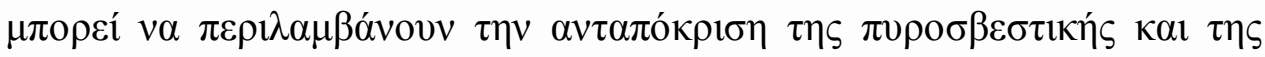

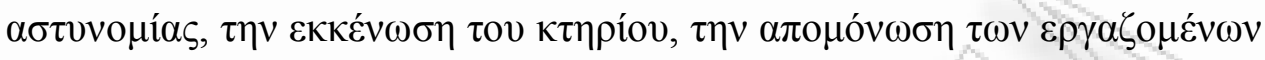

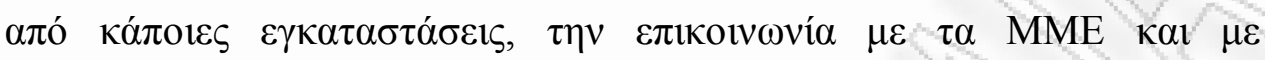

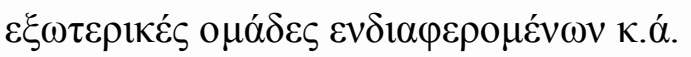

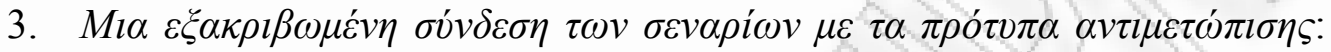

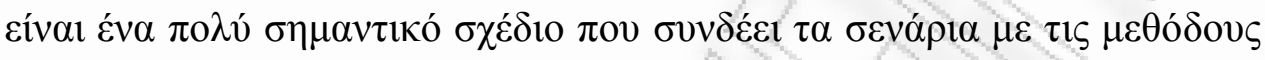

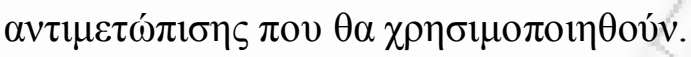

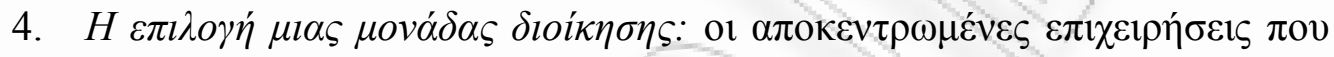

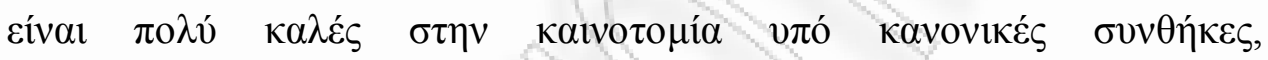

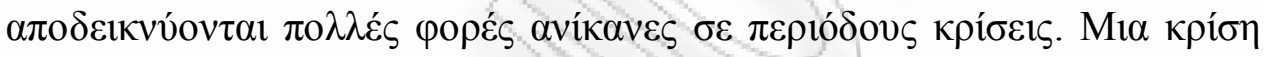

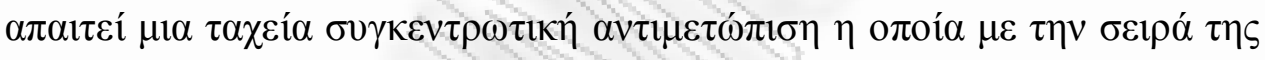

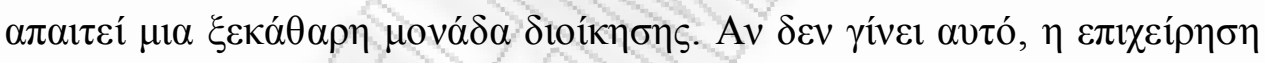

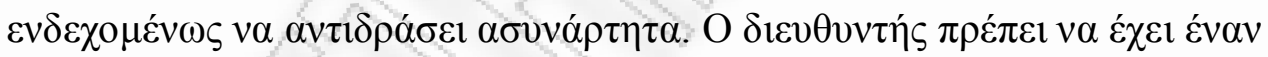

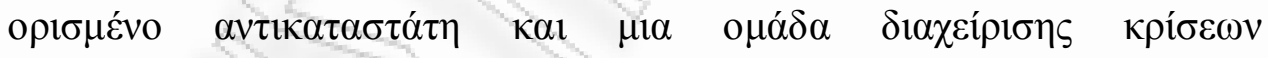

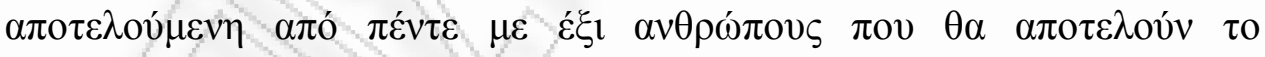

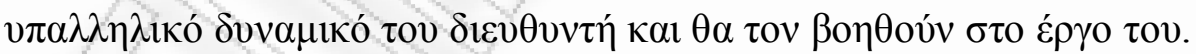

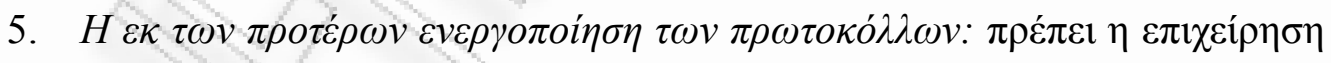

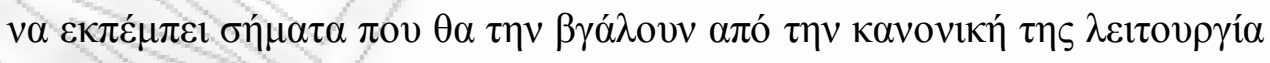

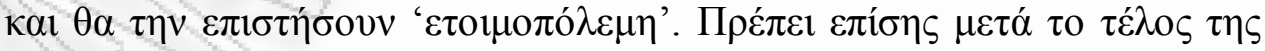

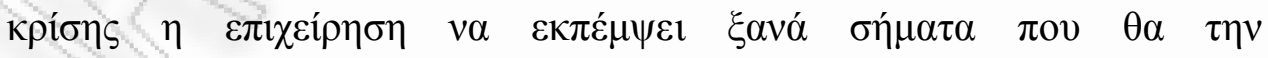

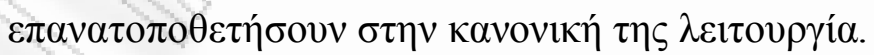

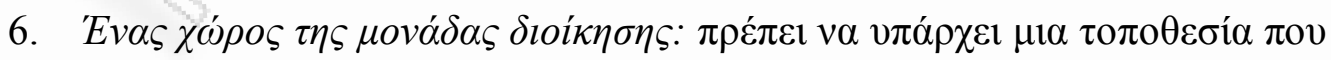

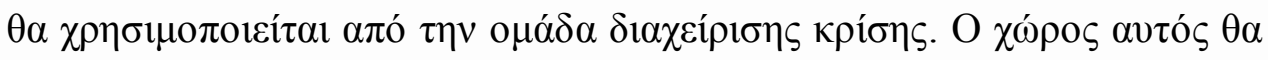

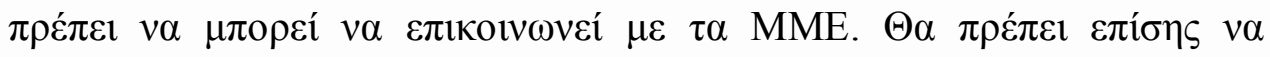




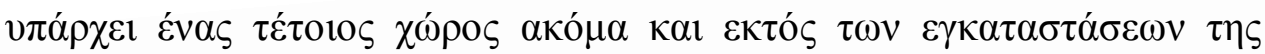

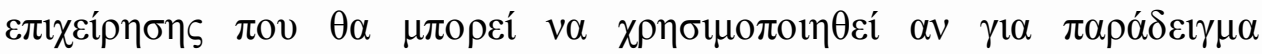

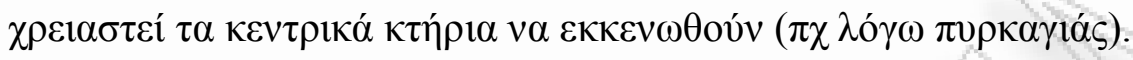

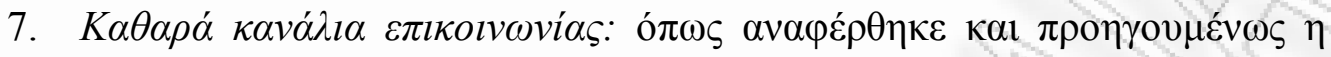

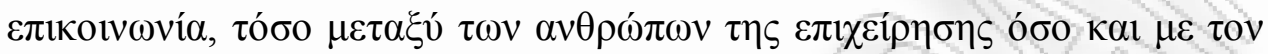

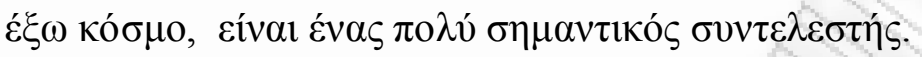

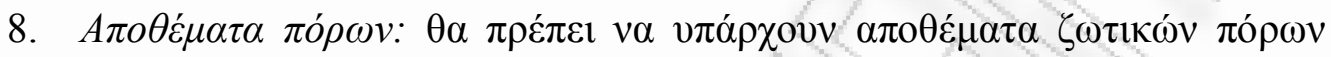

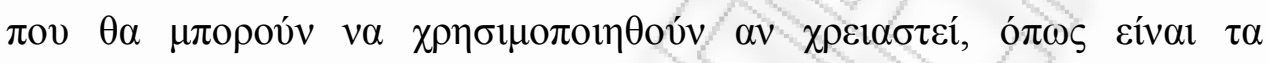

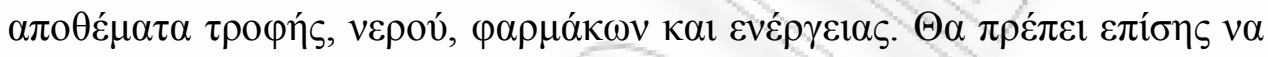

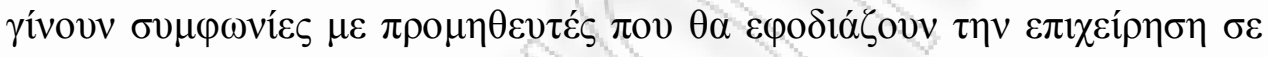

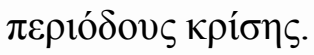

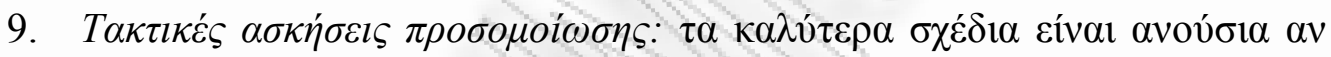

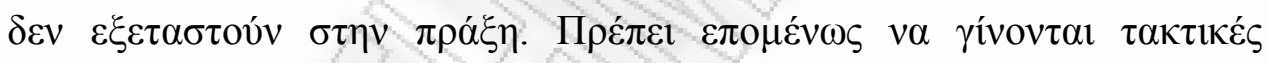
(

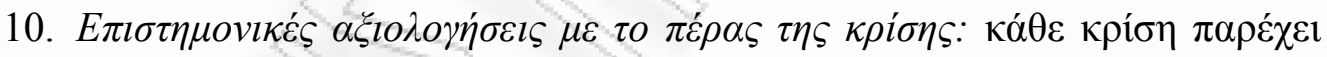

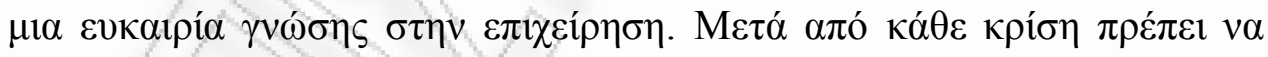

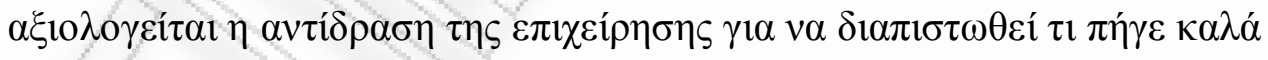

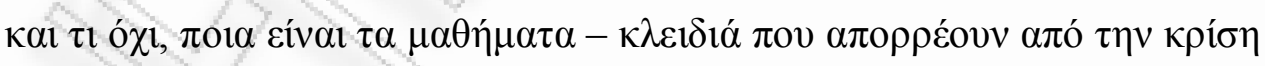

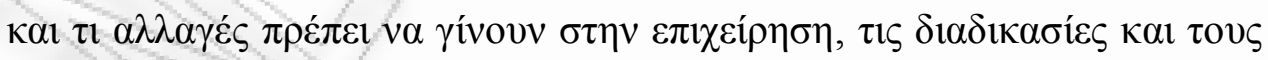
tópous.

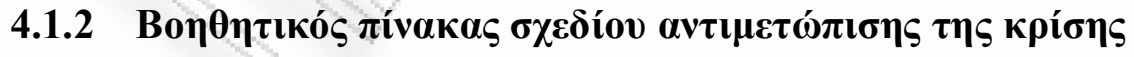

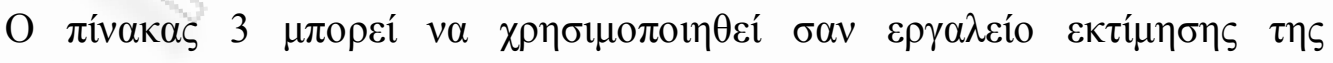

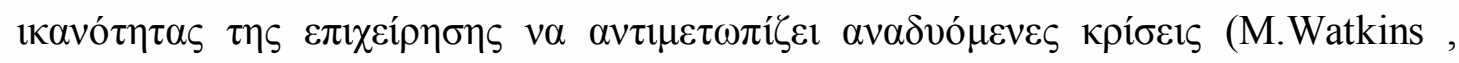
2001). 


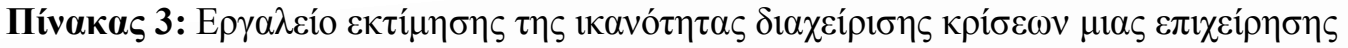

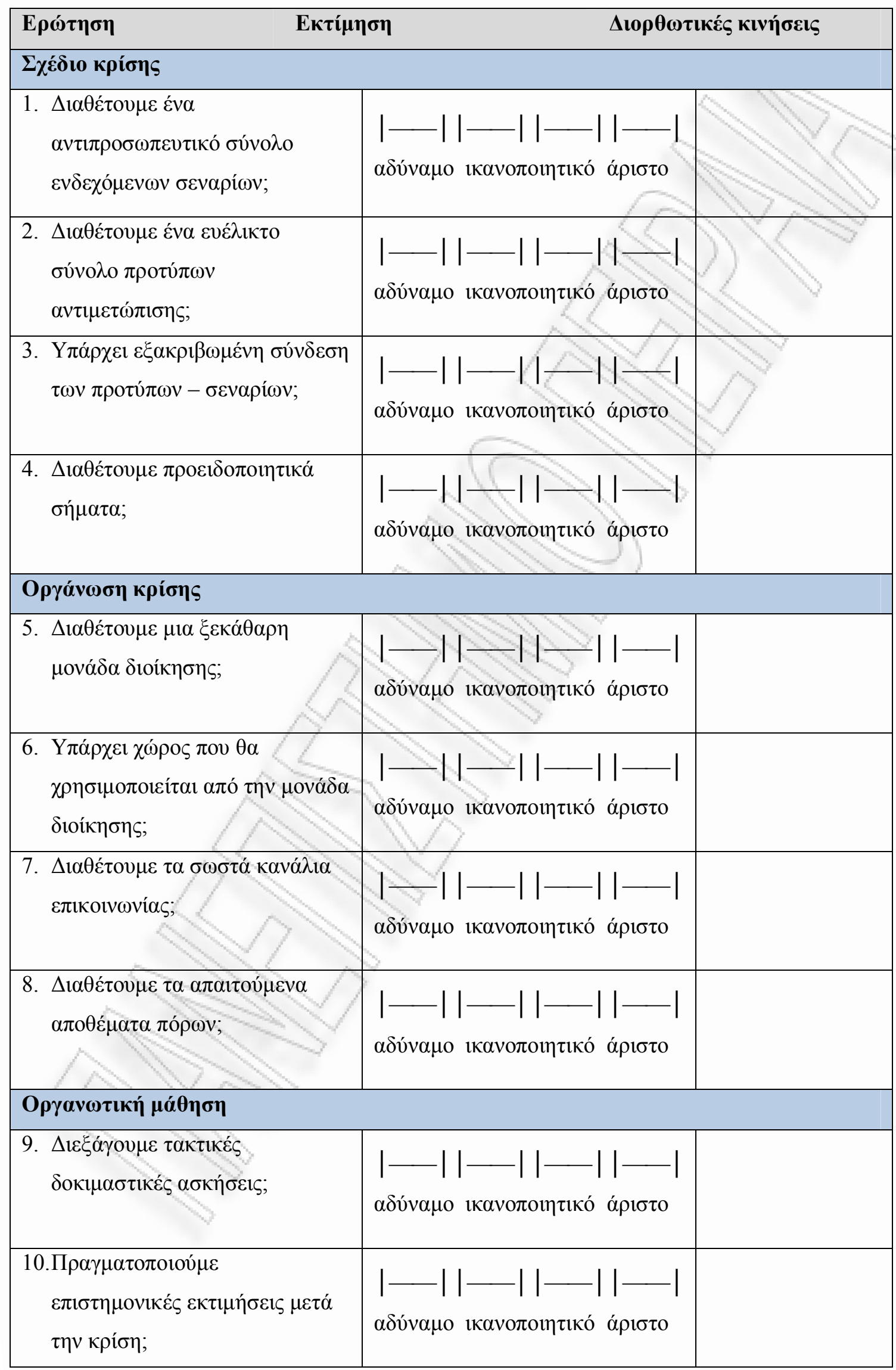




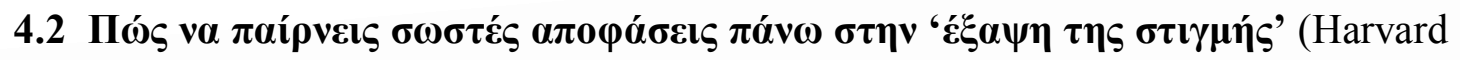
ManageMentor, 2005)

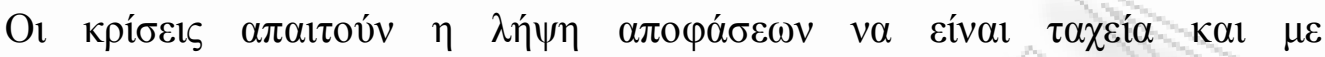

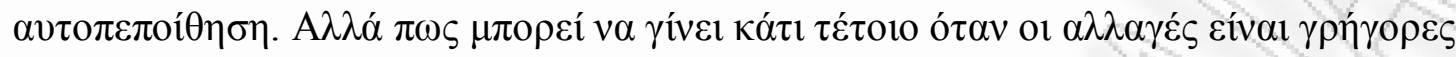

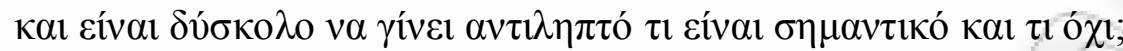

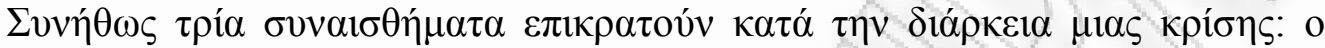

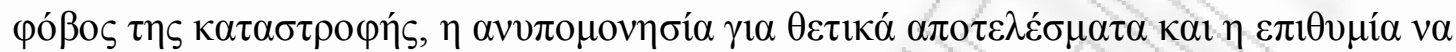

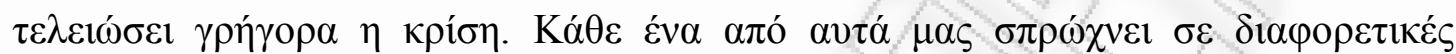

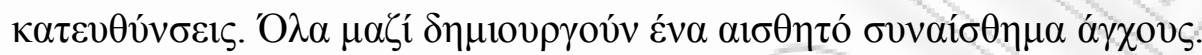

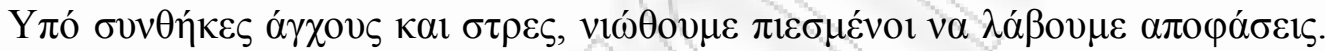

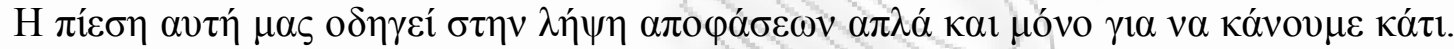

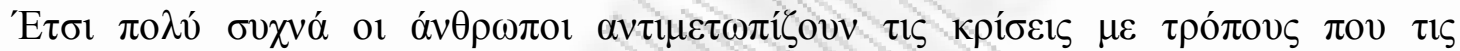

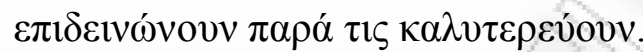

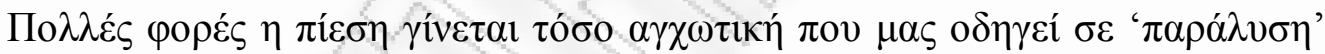

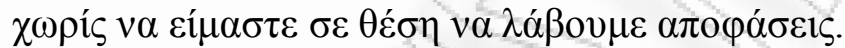

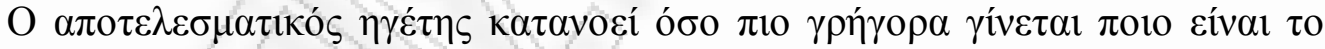

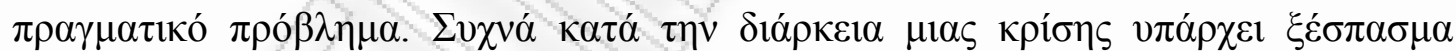
$\pi \lambda \eta \rho о \varphi о \rho ı \omega ́ v ~ \alpha \lambda \lambda \alpha$ ov $\pi \varepsilon \rho 1 \sigma \sigma o ́ \tau \varepsilon \rho \varepsilon \varsigma$ a

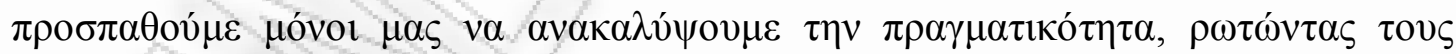

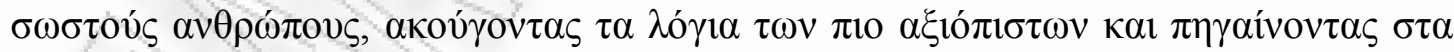
$\sigma \omega \sigma \tau \alpha \dot{\mu} \mu \dot{\varepsilon} \eta$.

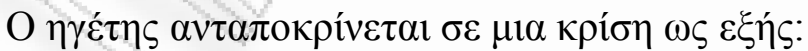

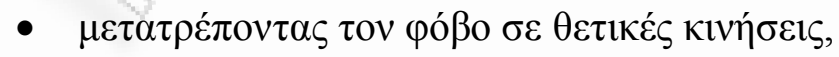

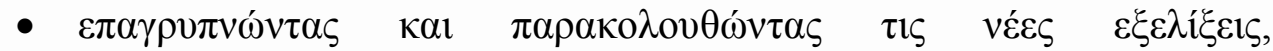

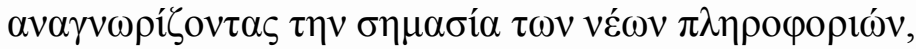




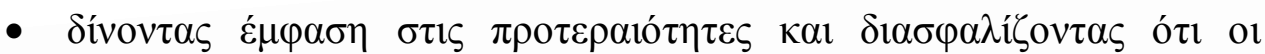

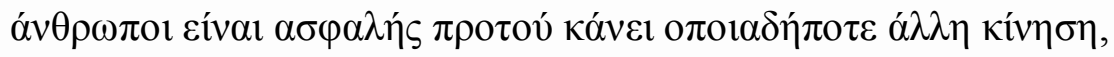

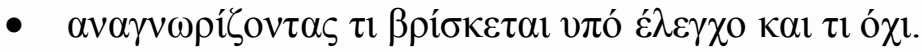

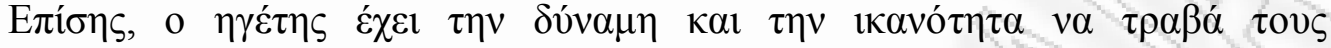

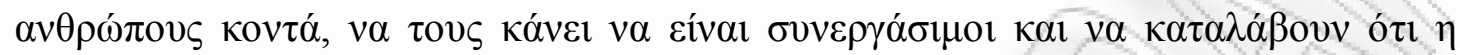

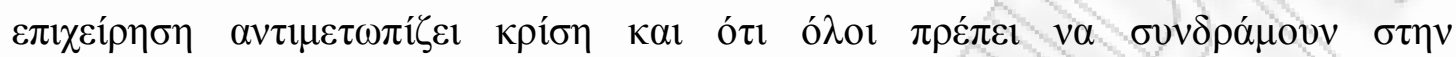

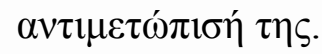

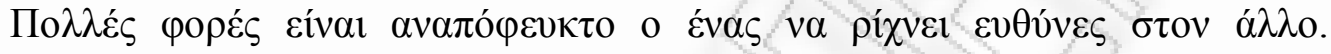

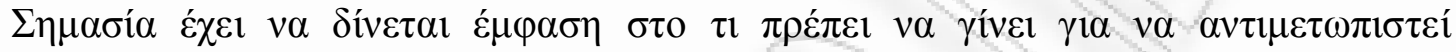

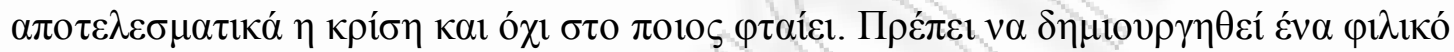

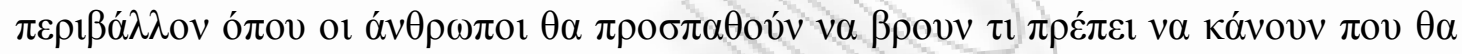

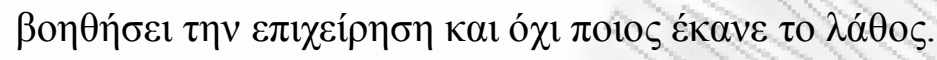

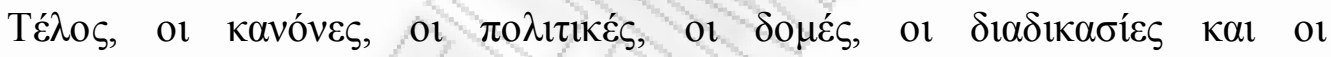

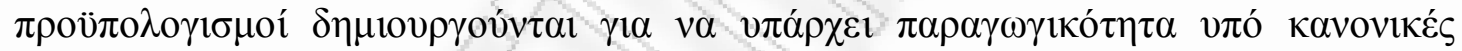

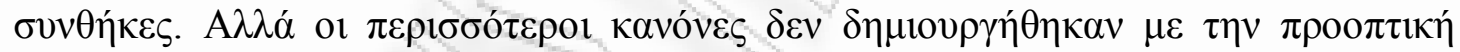

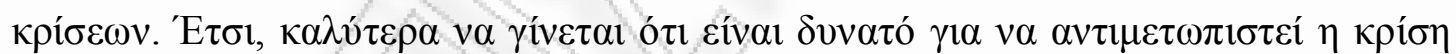

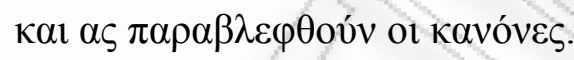




\section{MEPO $\Sigma$ ' $\Gamma$}

\section{RISK \& CRISIS MANAGEMENT: $\Sigma Y N \Delta Y A \Sigma M O \Sigma$}

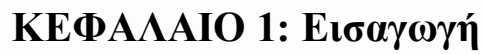

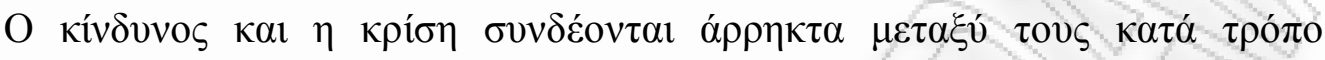

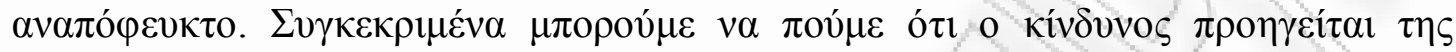

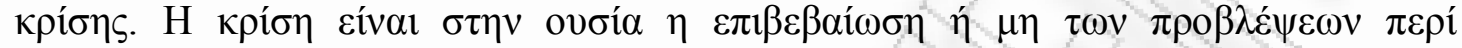

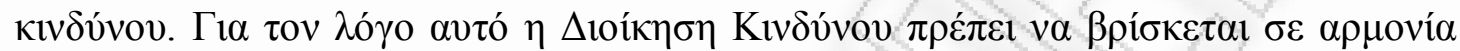

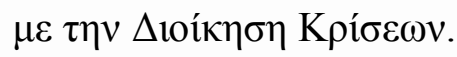

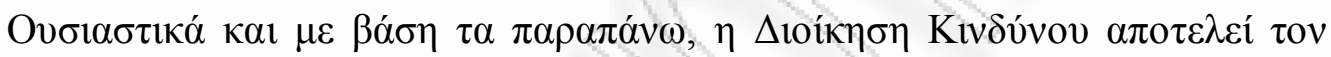

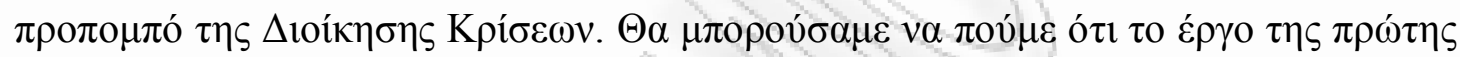

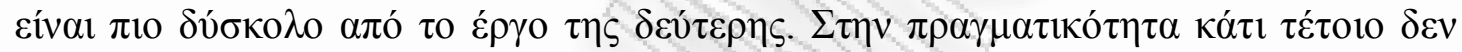

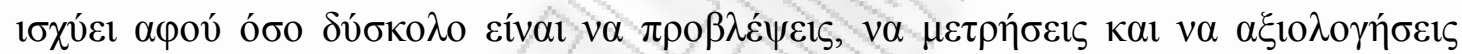

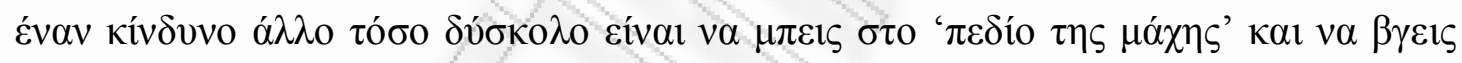

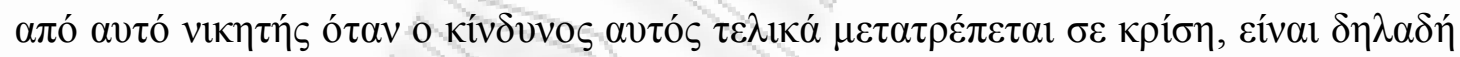

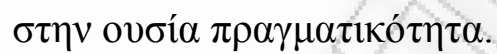

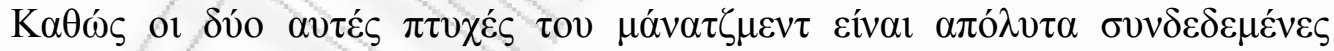

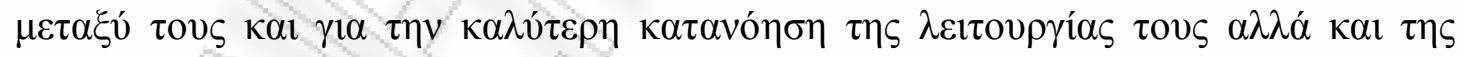

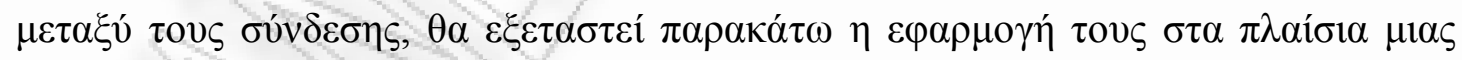

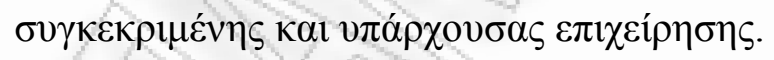




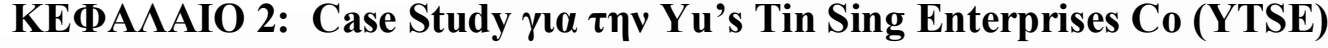

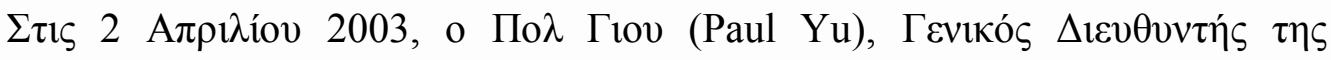

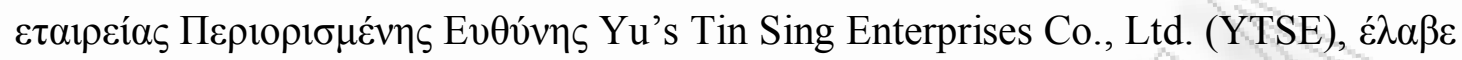

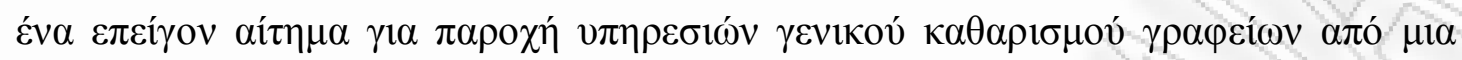

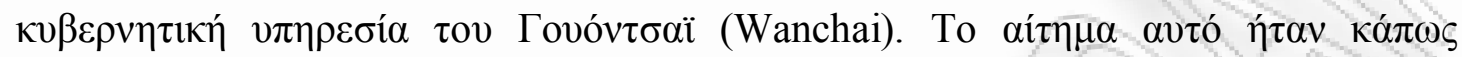

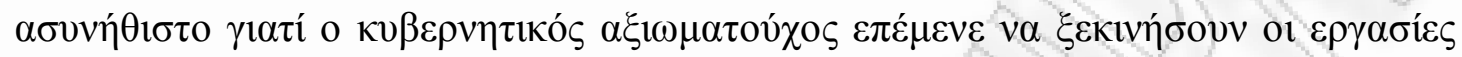

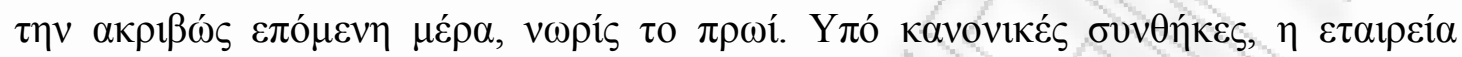

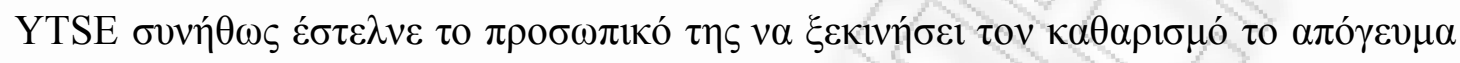

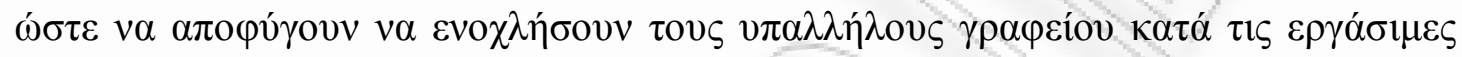


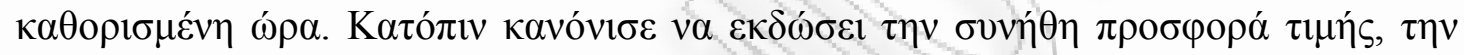

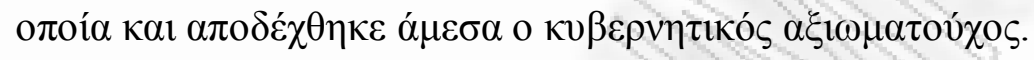

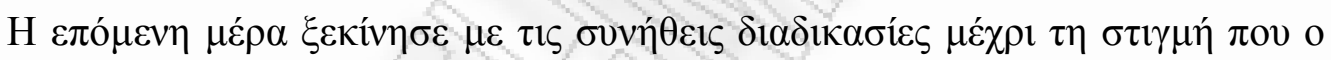

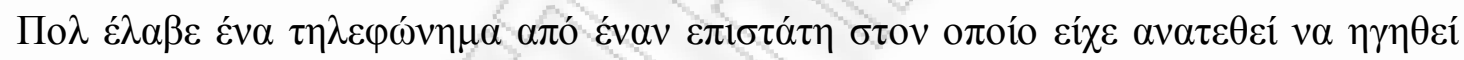

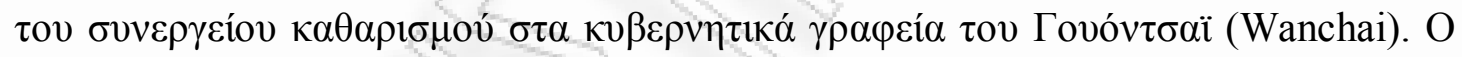

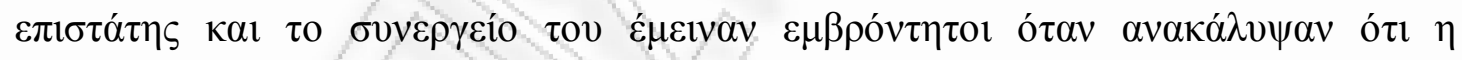

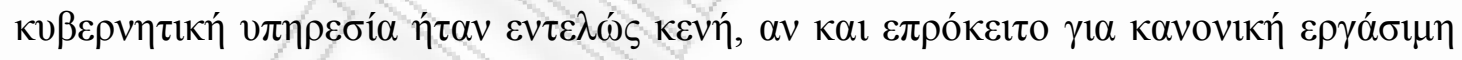

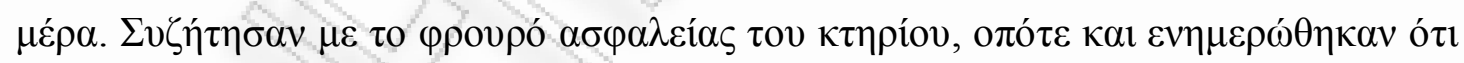

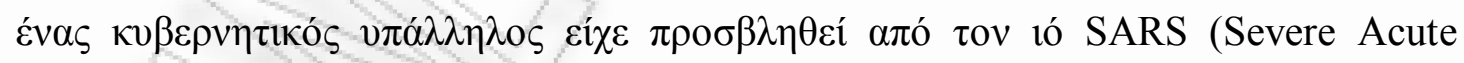

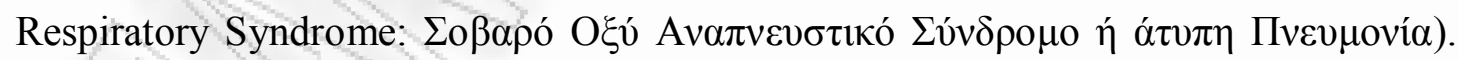

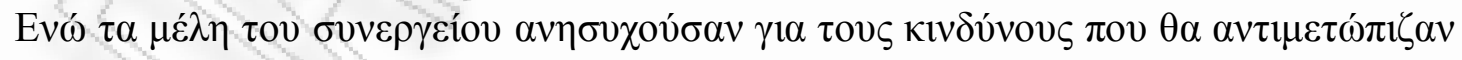

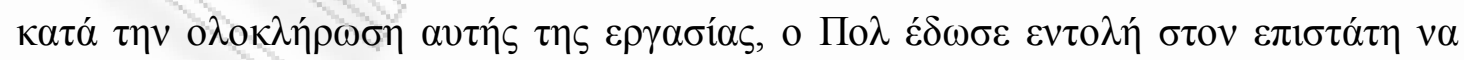

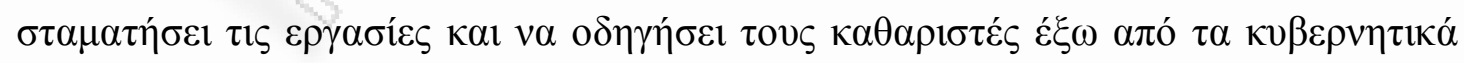

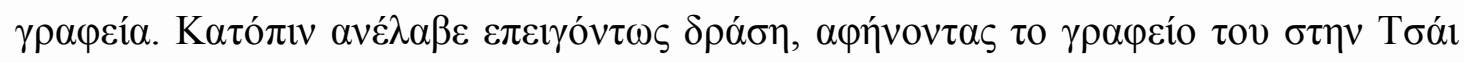

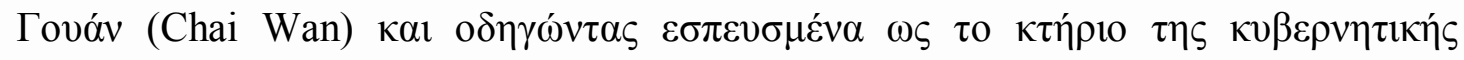




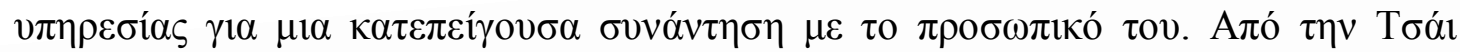

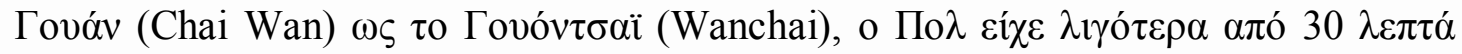

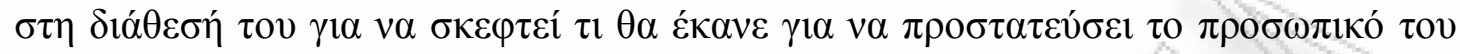

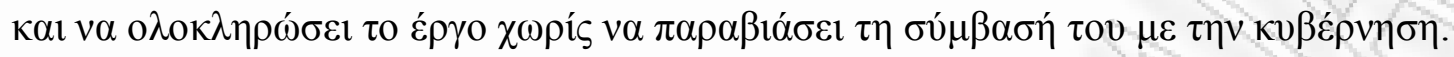

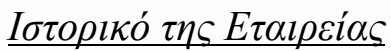

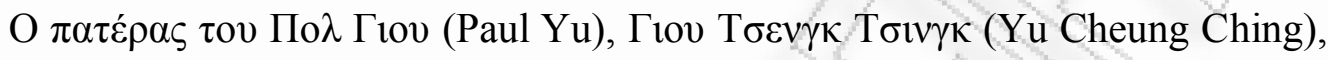

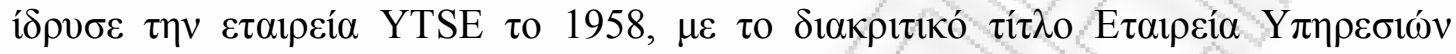

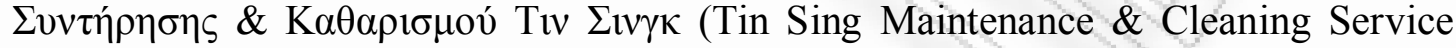

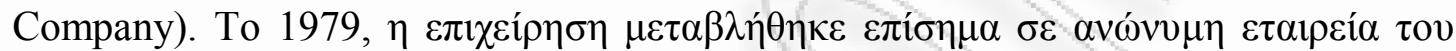

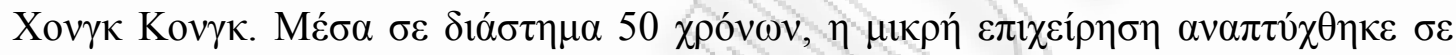

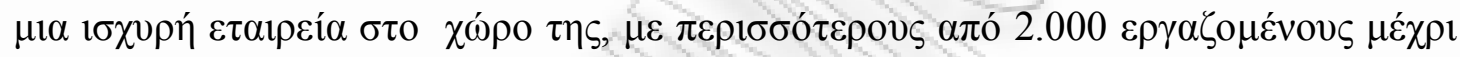

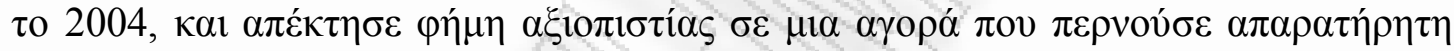

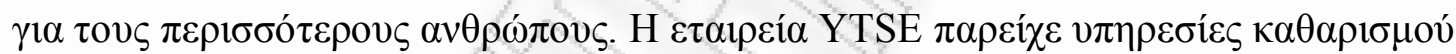

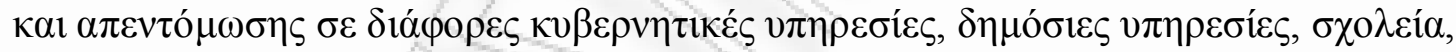

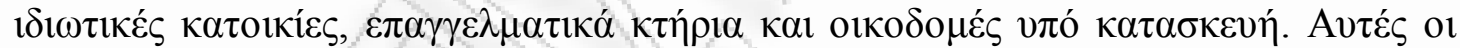

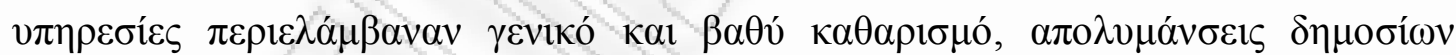

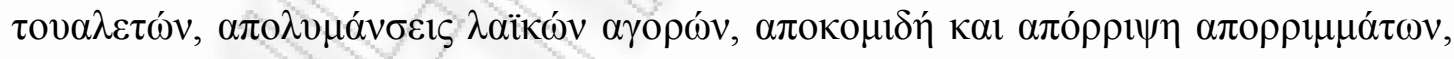

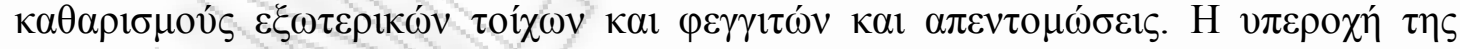

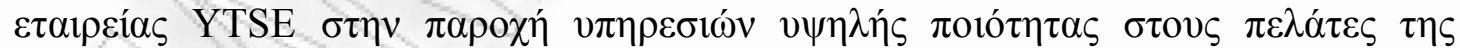

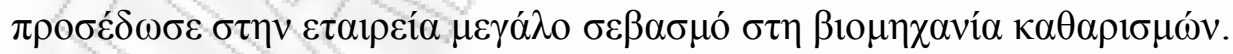

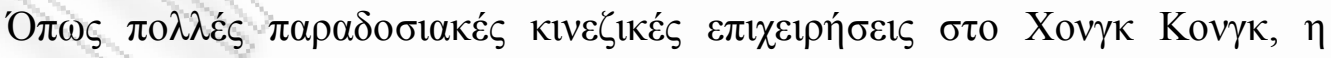

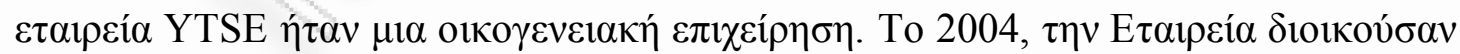

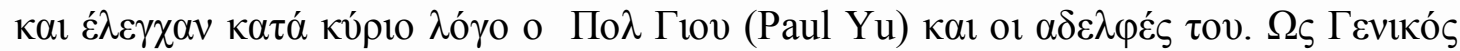

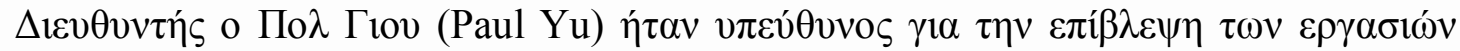




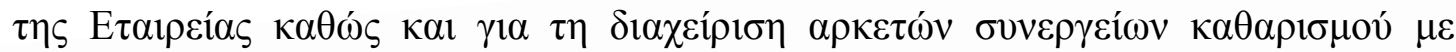

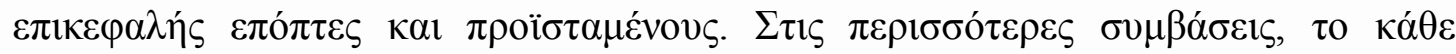

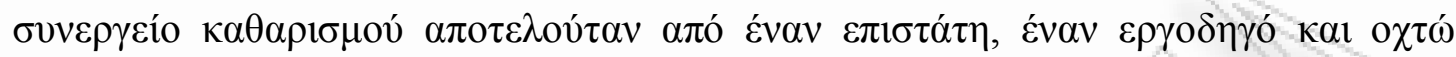

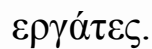

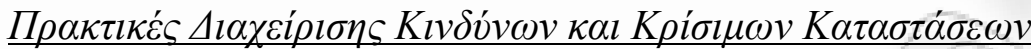

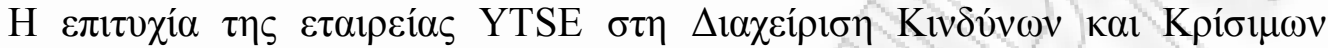

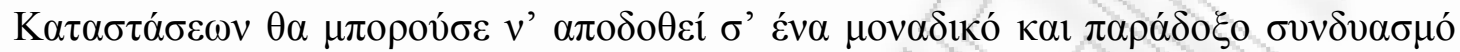

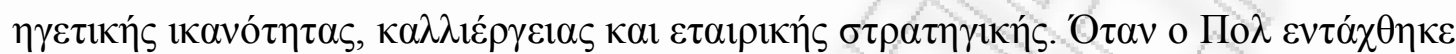

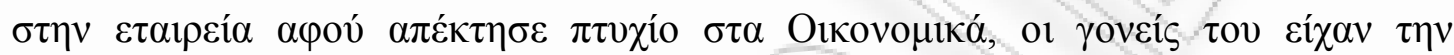

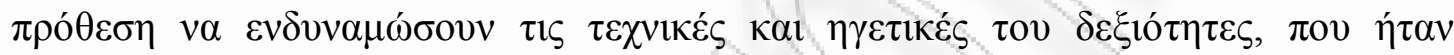

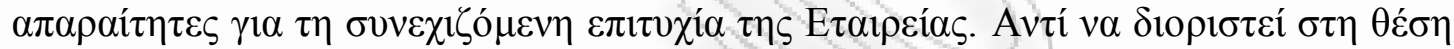

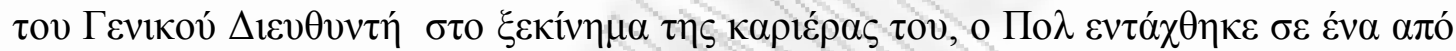

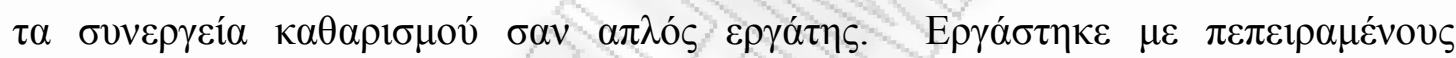

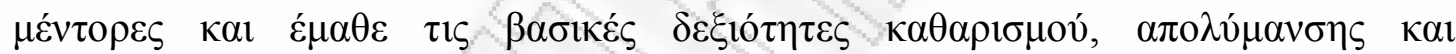

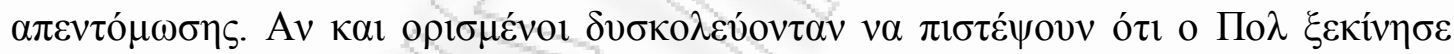

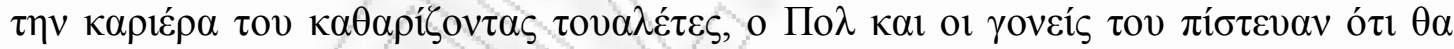

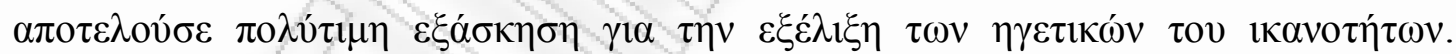

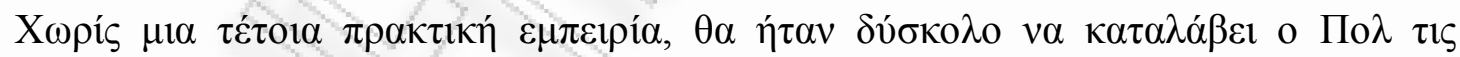

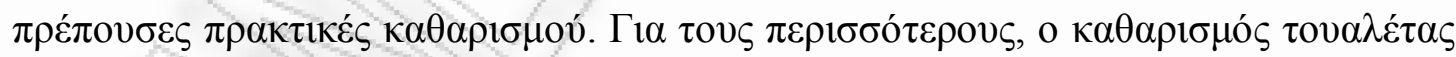

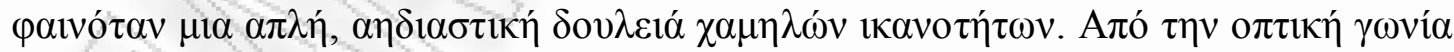

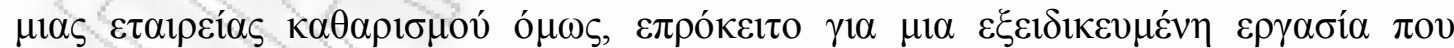

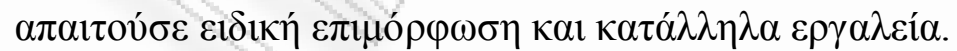

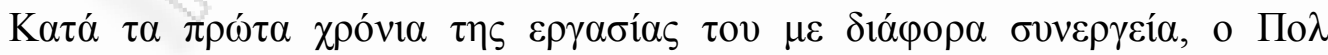

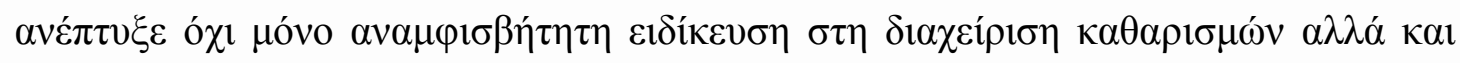

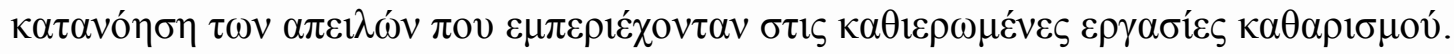




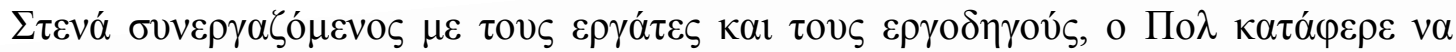

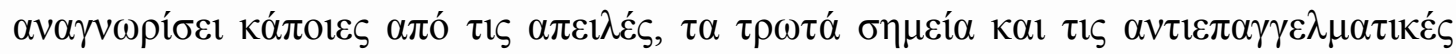

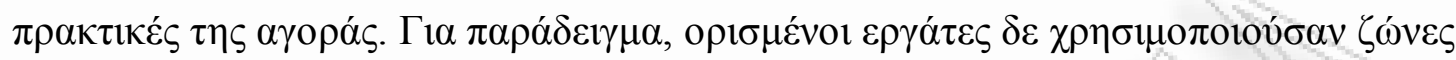

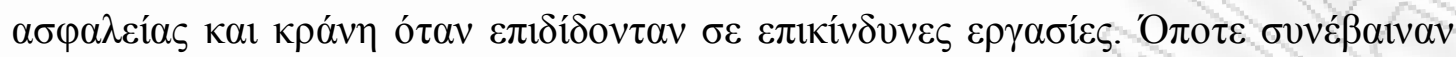

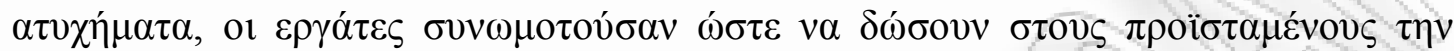

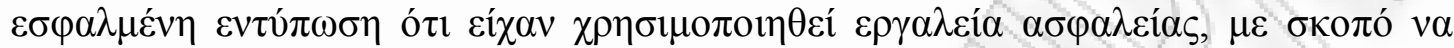

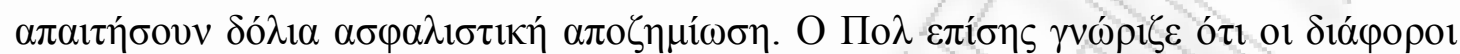

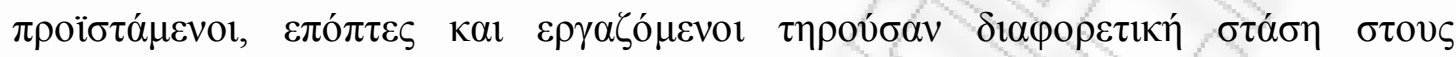

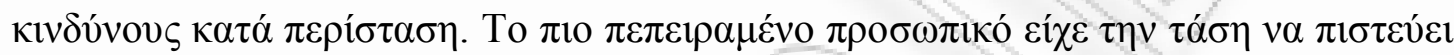

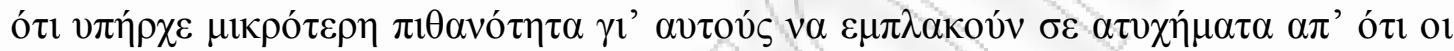

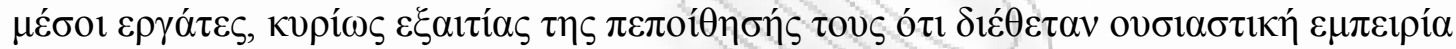

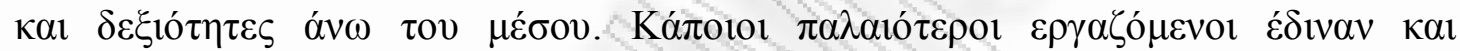

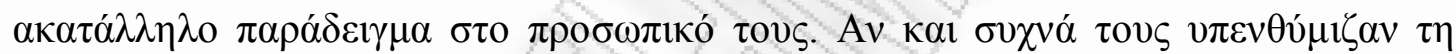

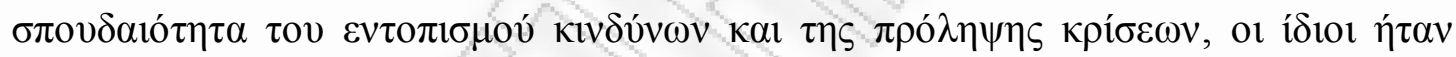

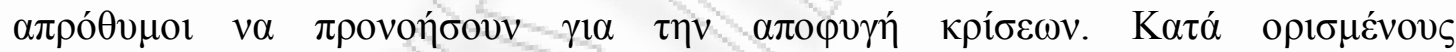

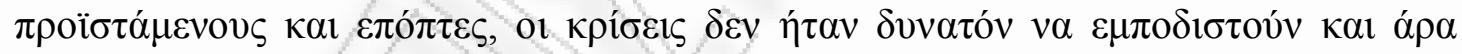

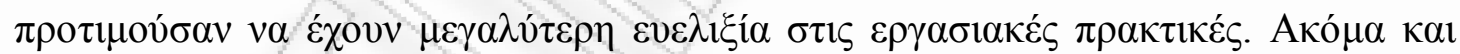

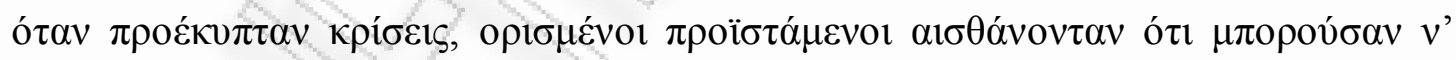

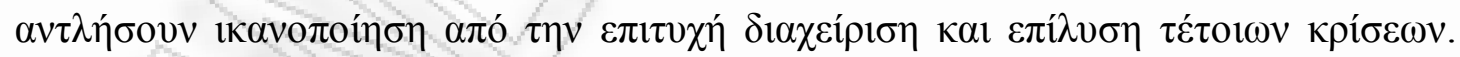

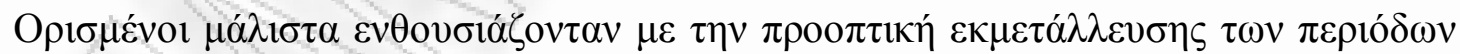

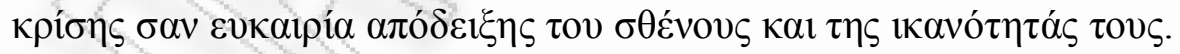




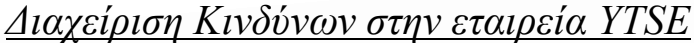

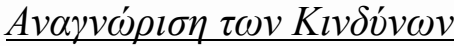

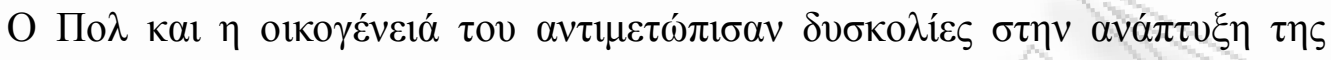

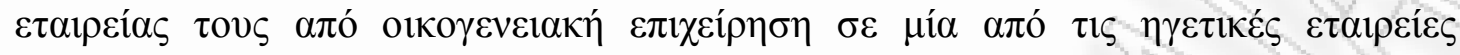

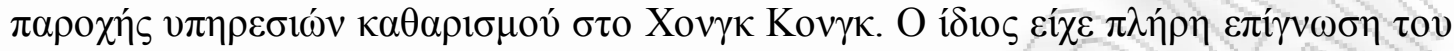

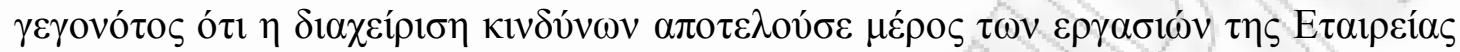

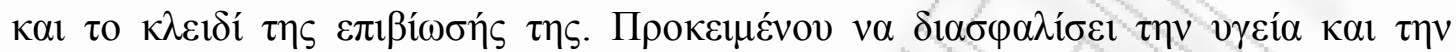

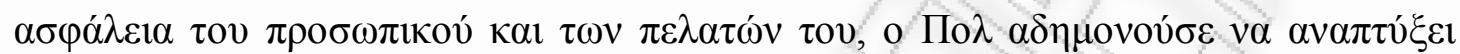

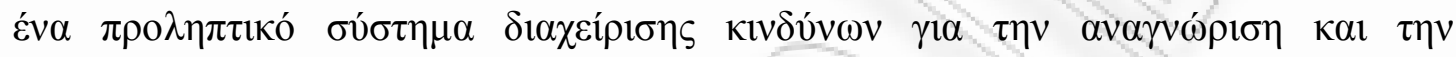

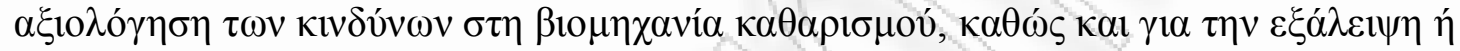

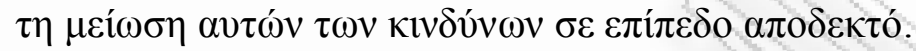

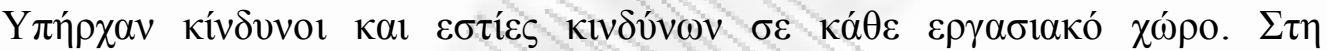

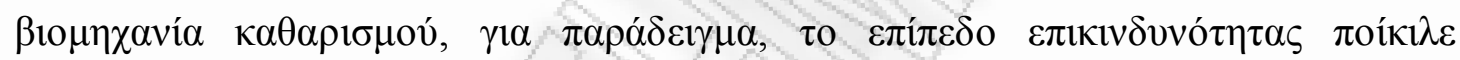

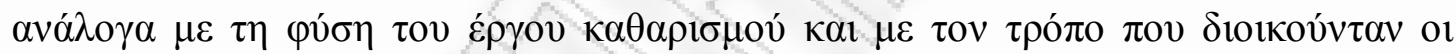

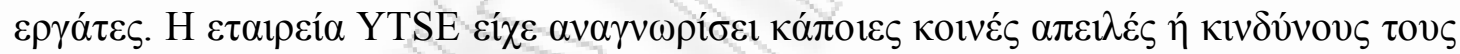

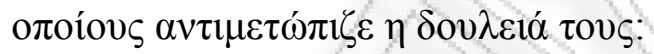

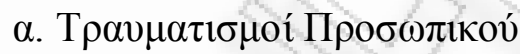

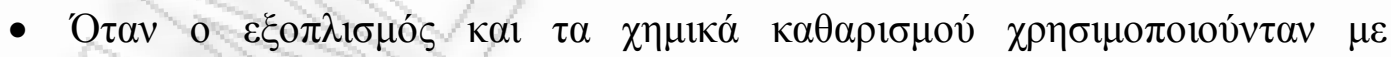

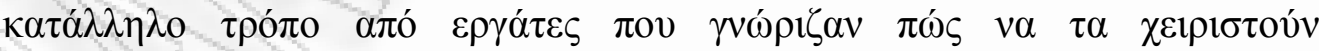

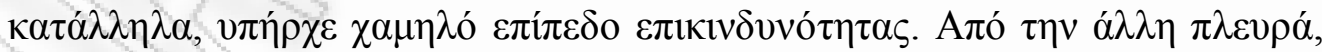

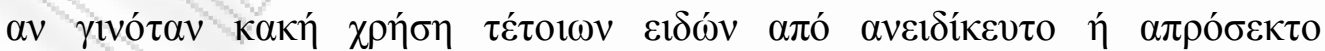

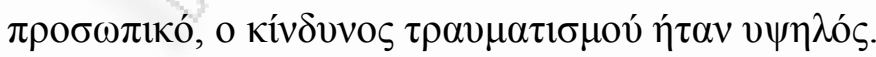

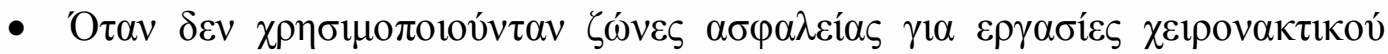

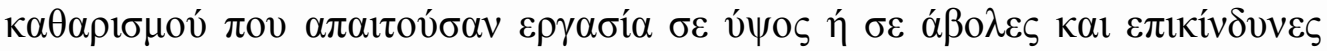




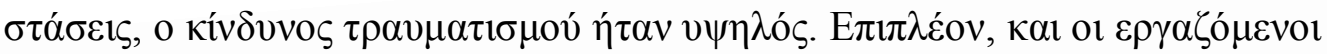

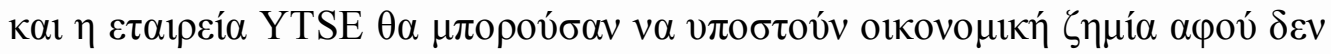

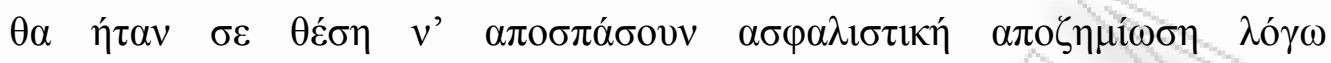

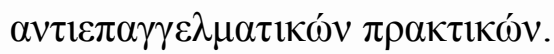

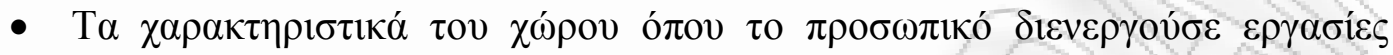

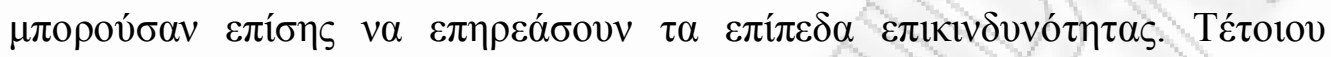

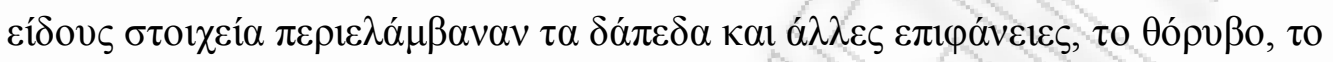

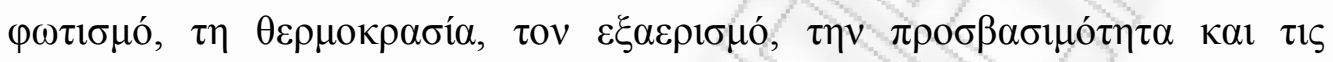

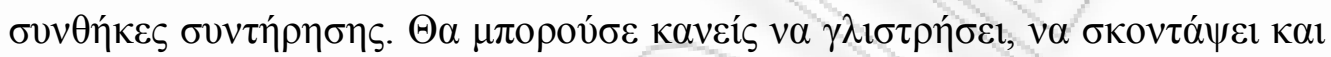

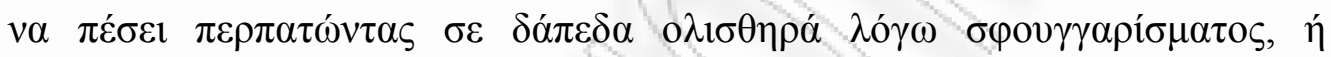

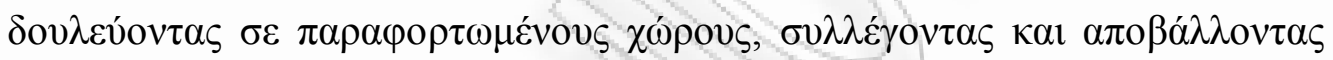

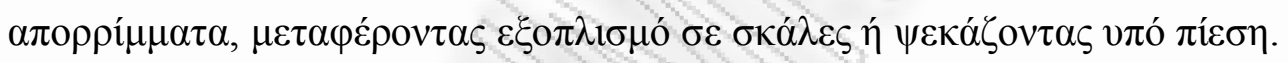

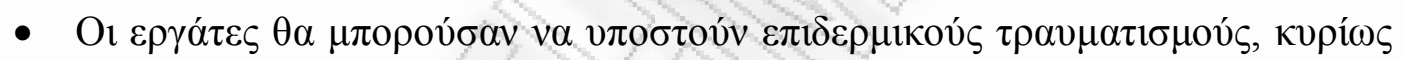

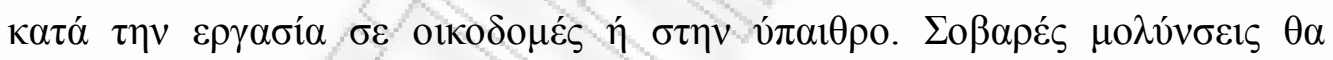

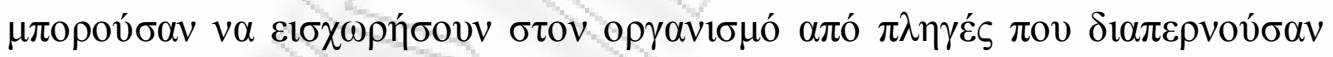

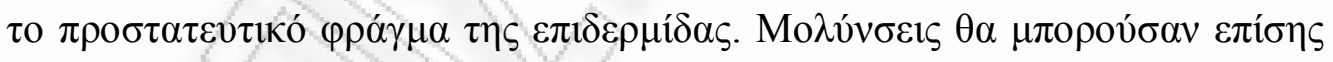

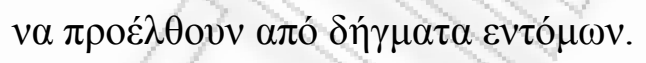

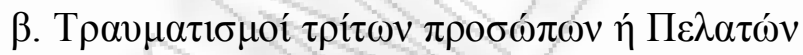

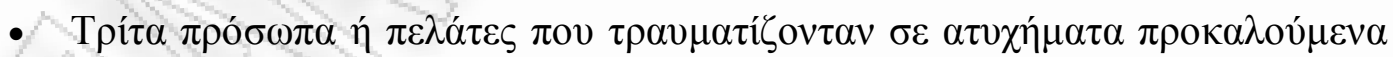

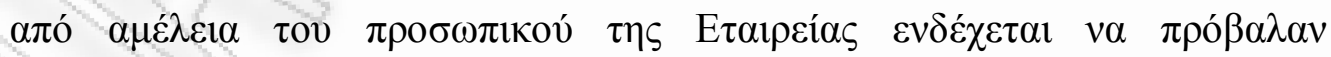

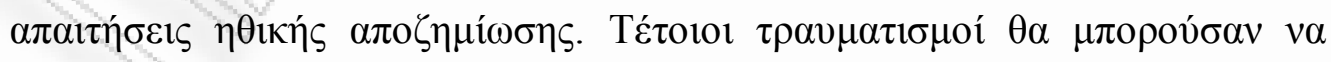

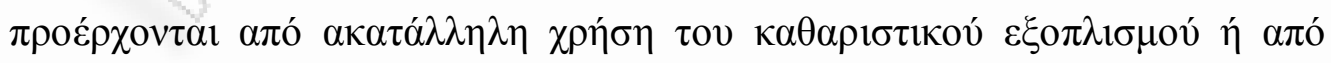

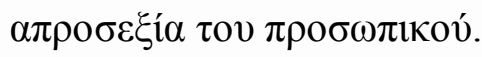




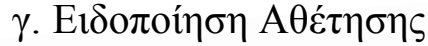

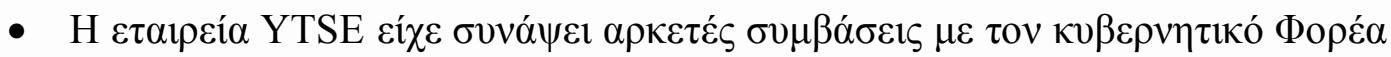

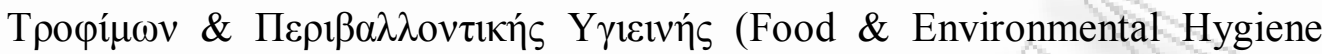

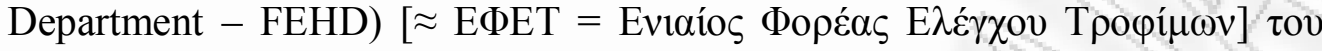

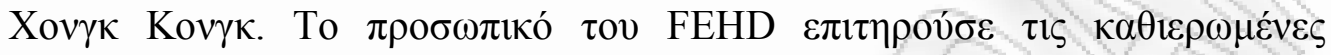

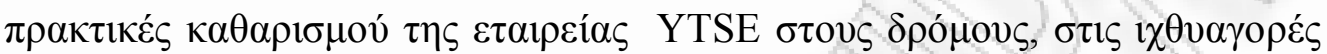

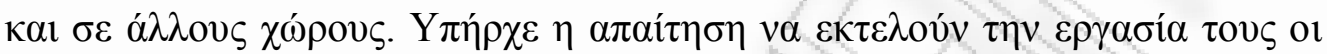

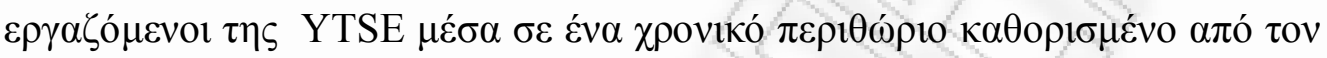

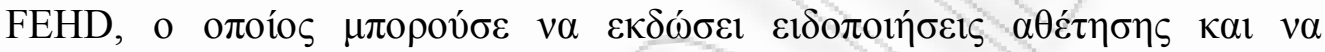

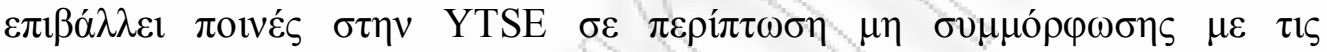

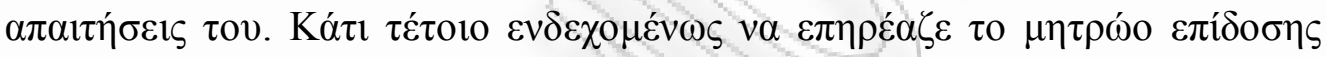

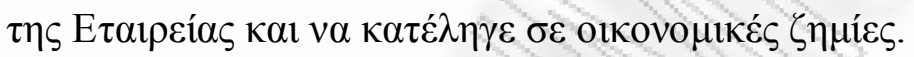

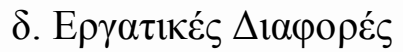

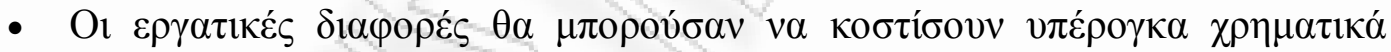
$\pi$

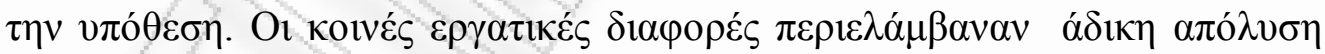

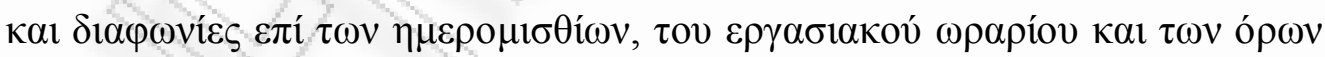

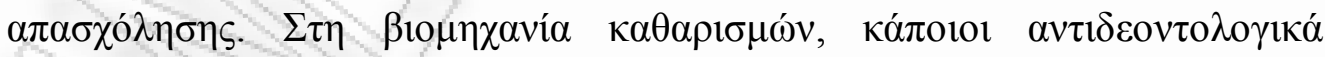

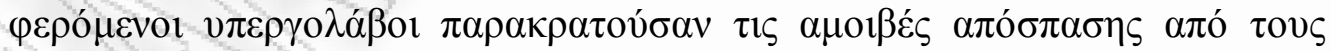

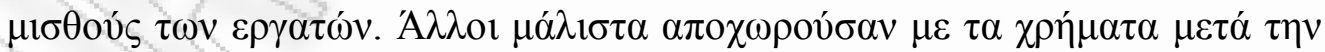

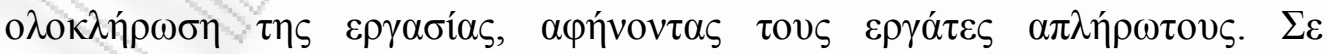

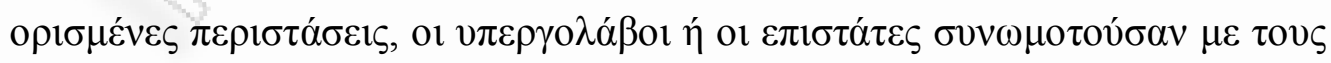

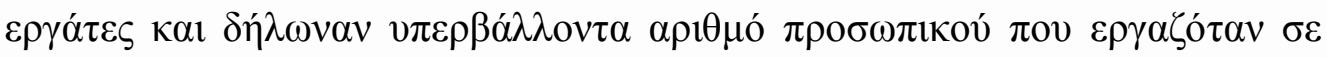

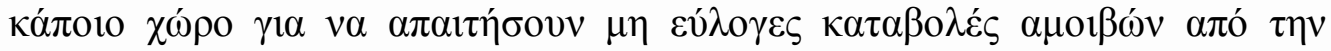




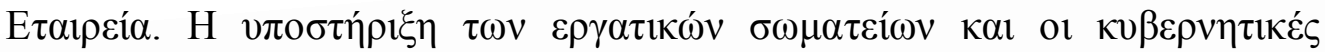

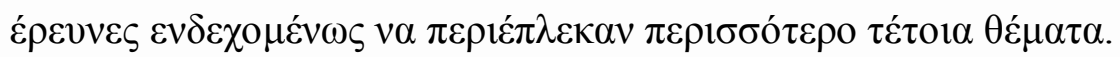

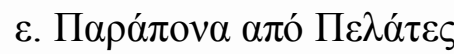

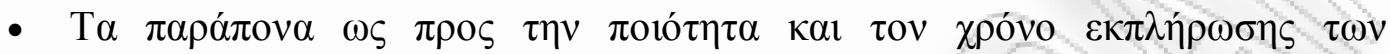

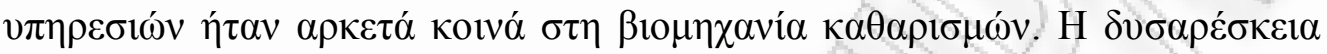

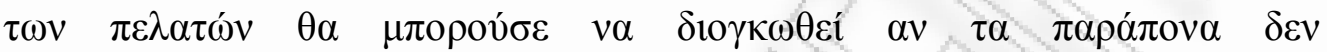

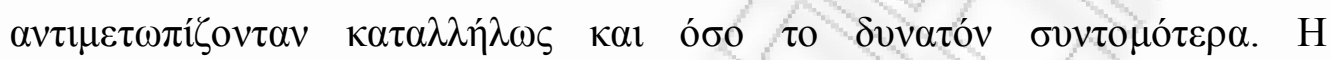

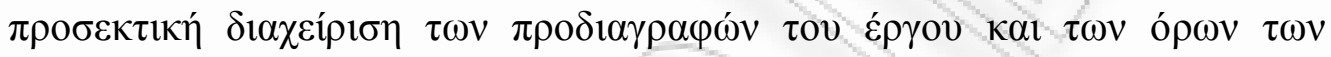

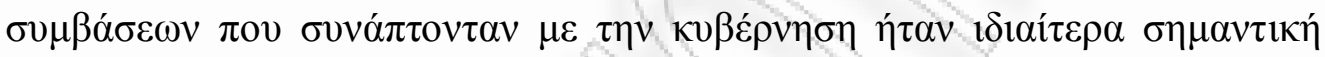

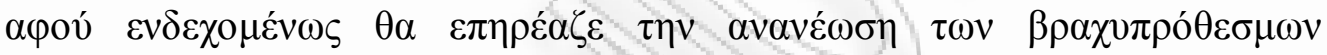

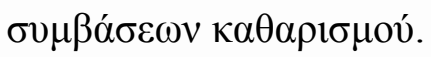

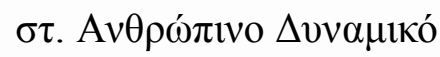

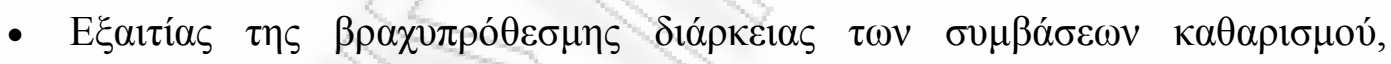

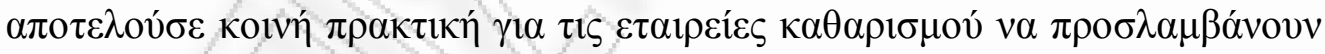

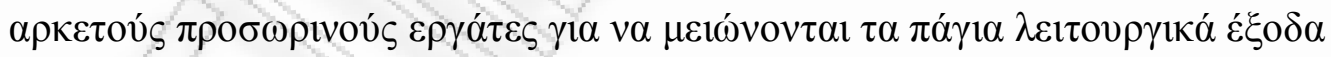

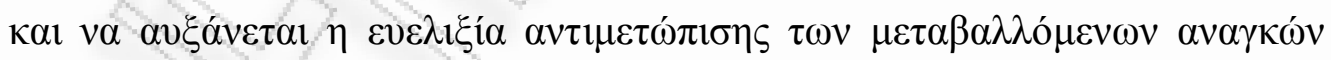

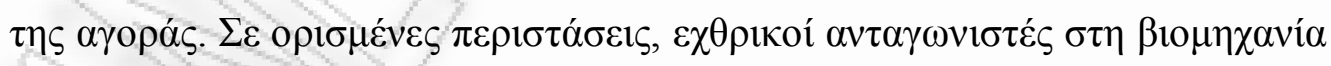

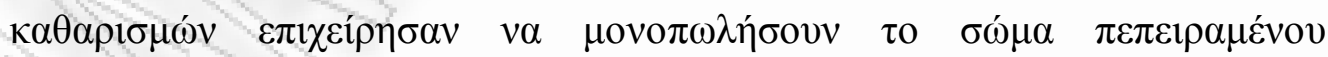

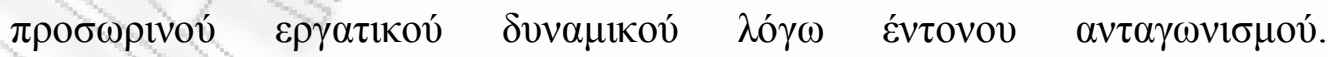

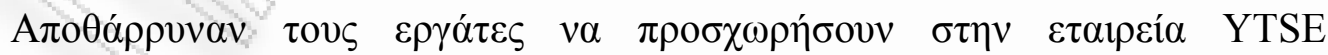

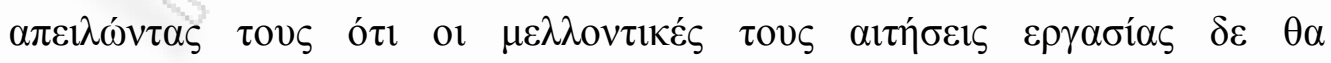

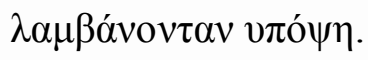




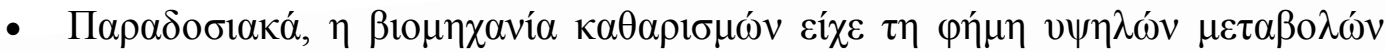

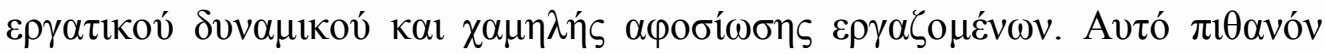

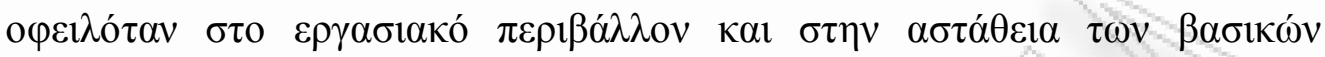

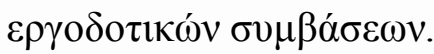

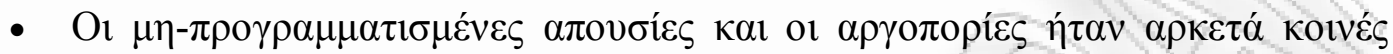
$\mu \varepsilon \tau \alpha \xi \tilde{v} \pi \rho \circ \sigma \omega \rho \imath v \omega ́ v \kappa \alpha \theta \alpha \rho \imath \sigma \tau \omega ́ v$.

\section{$\underline{\sum \tau \rho \alpha \tau \eta \gamma l \kappa \varepsilon ́ \varsigma ~ \Delta l \alpha \chi \varepsilon i ́ \rho l \sigma \eta \varsigma ~ K l v \delta v ́ v o v ~}$}

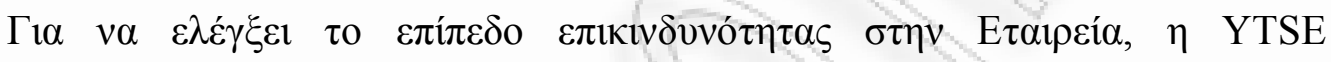

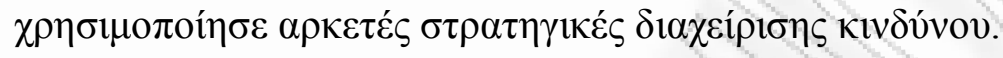

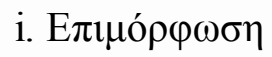

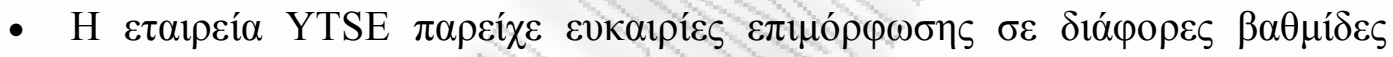

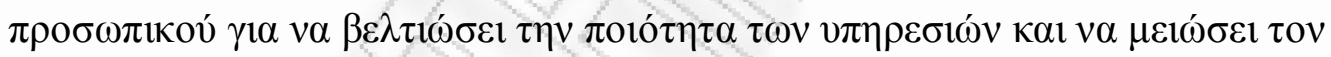

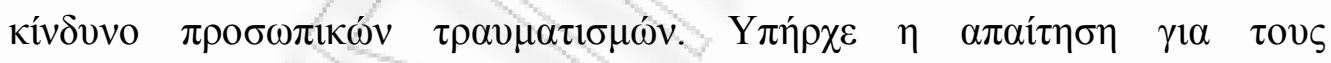

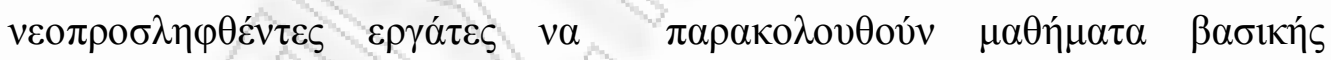

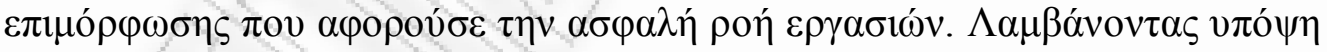

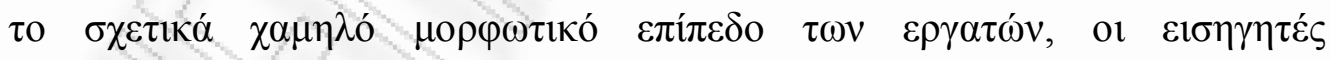

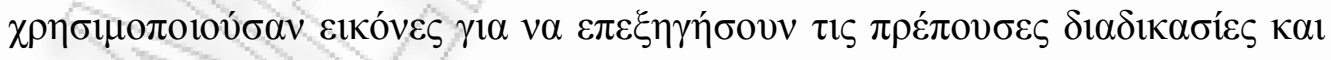

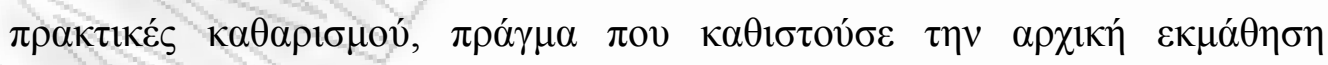

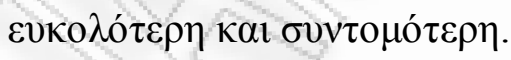

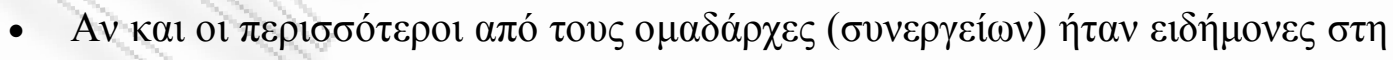

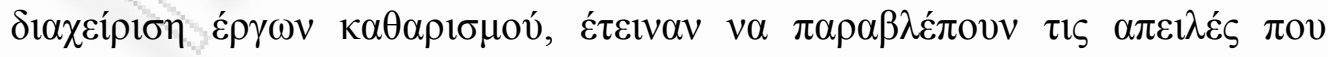

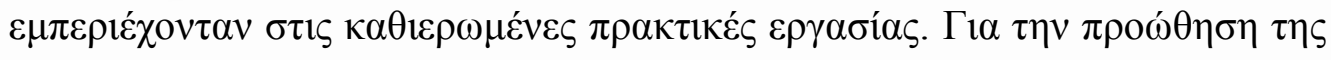

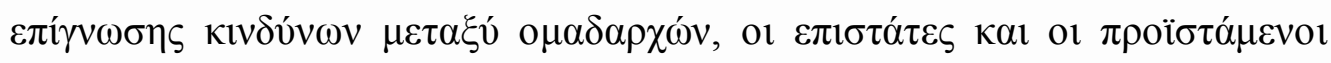

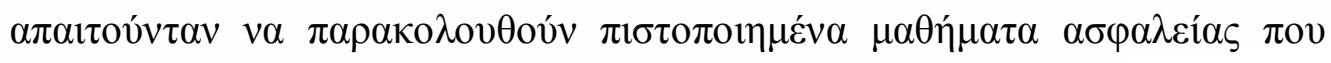




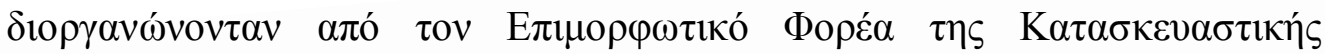

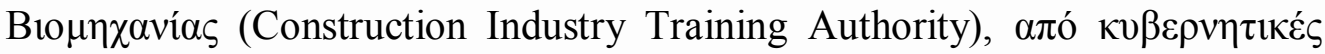

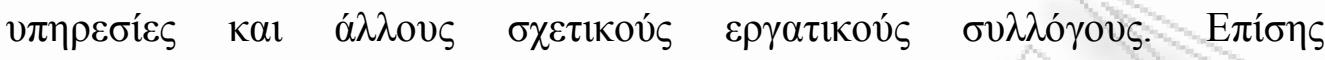

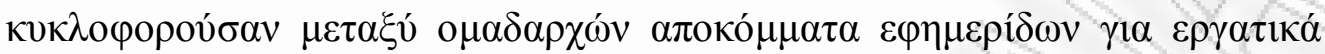

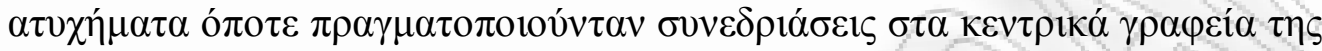
Eqaipsías.

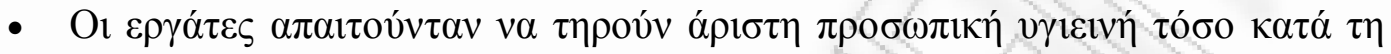

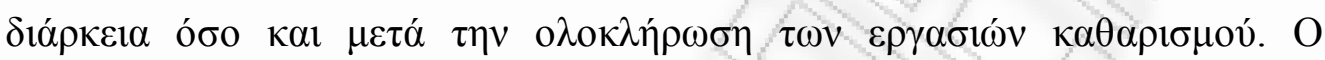

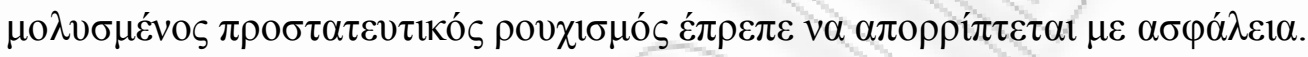

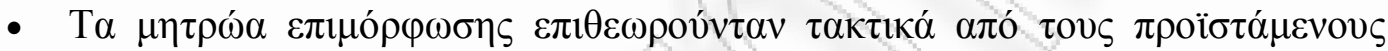

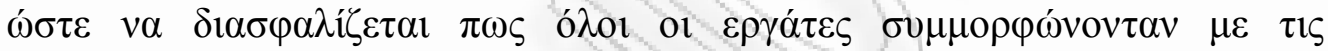
$\alpha \pi \alpha \imath \eta ́ \sigma \varepsilon 1 \varsigma \varepsilon \pi \mu o ́ \rho \varphi \omega \sigma \eta \varsigma$.

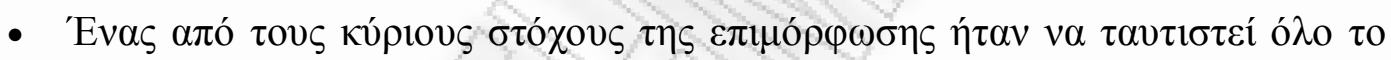

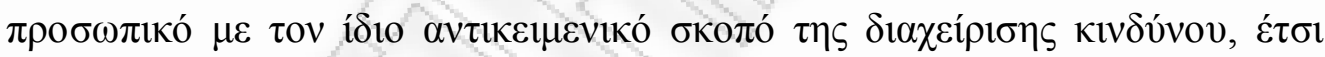

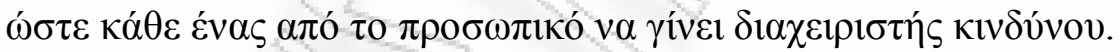

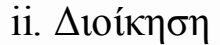

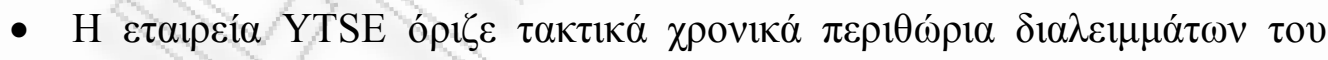

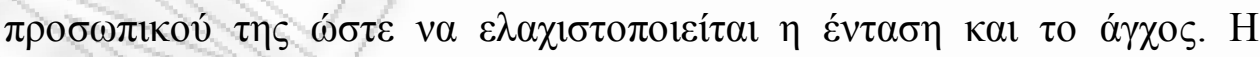

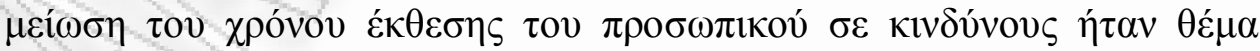

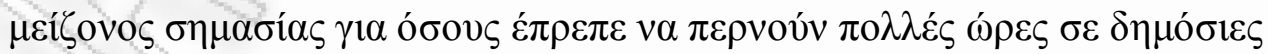

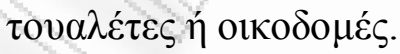

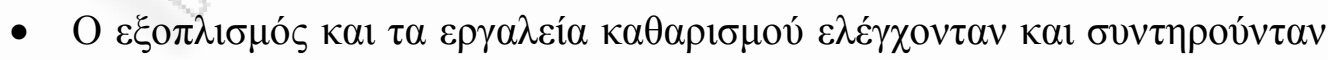

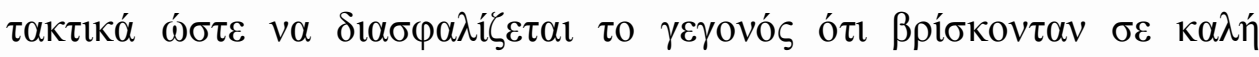
$\kappa \alpha \tau \alpha ́ \sigma \tau \alpha \sigma \eta$. 


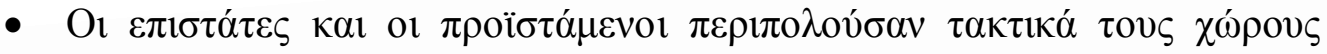

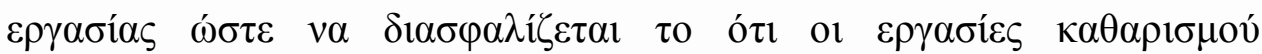

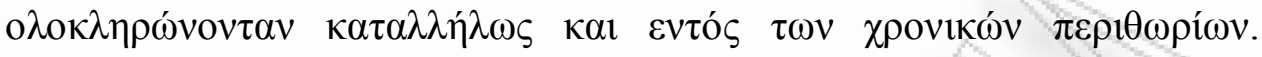

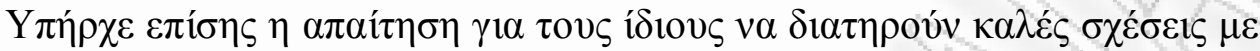

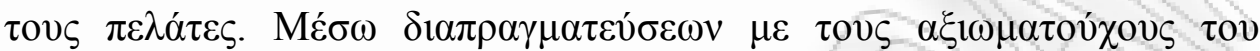

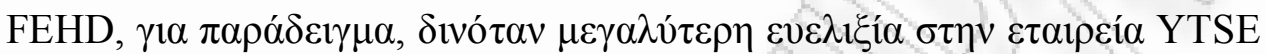

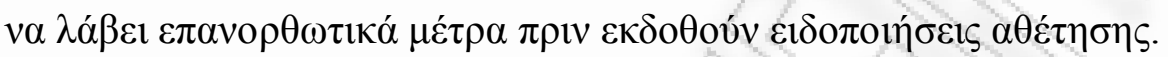

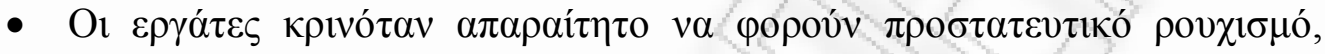

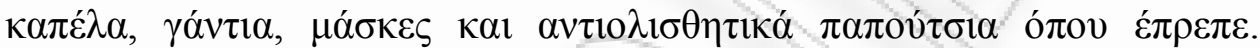

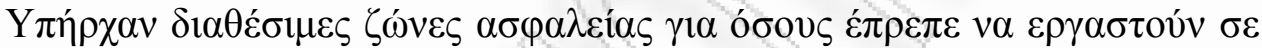

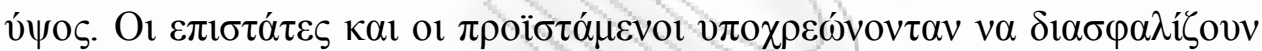

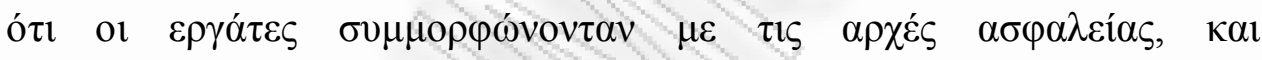

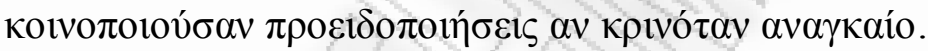

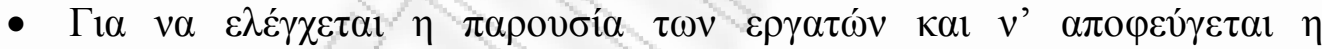

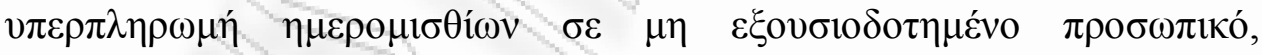

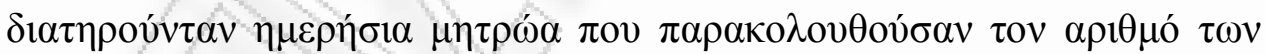

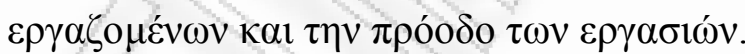

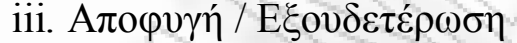

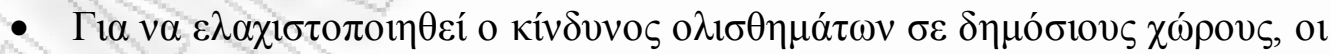

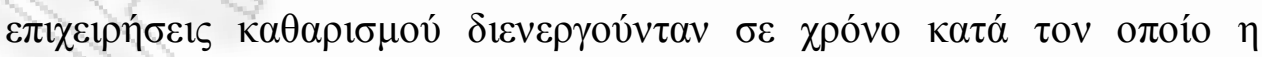

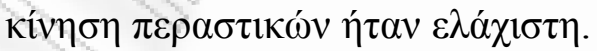

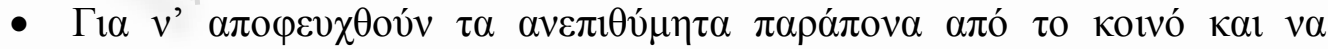

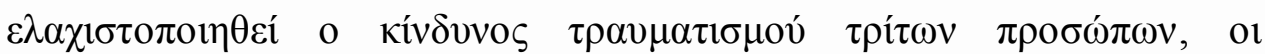

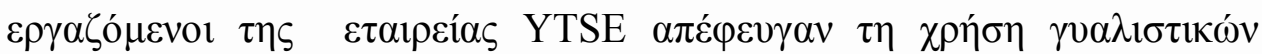




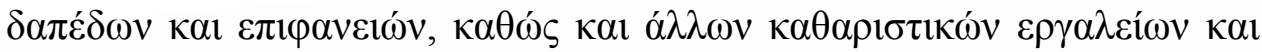

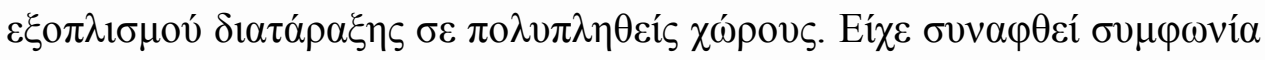

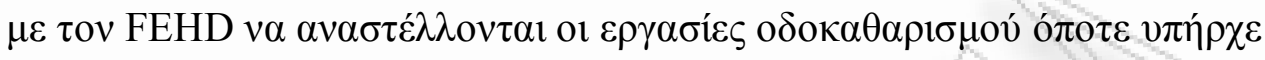

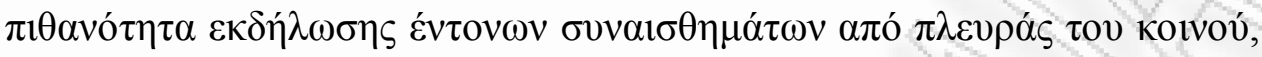

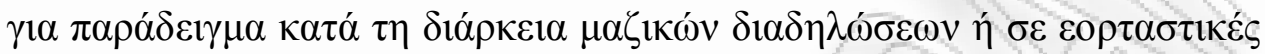

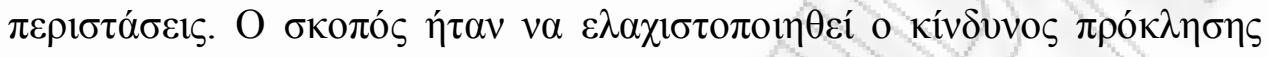

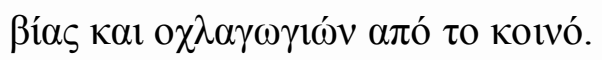

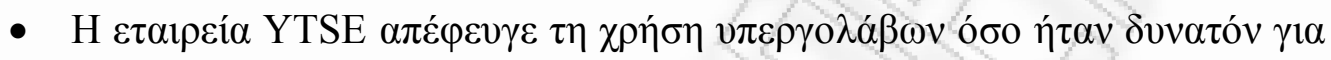

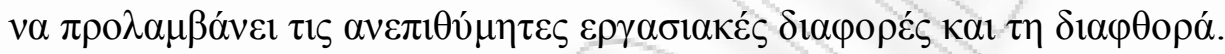

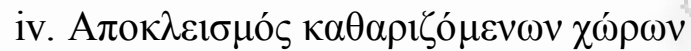

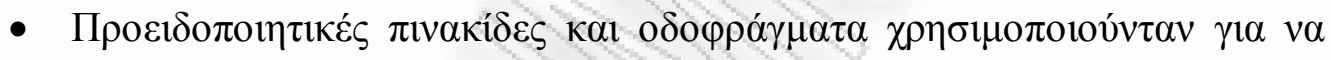

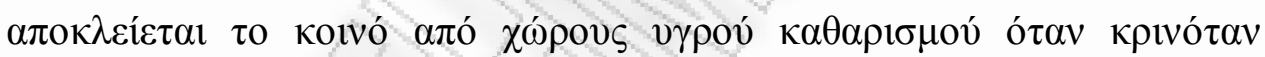

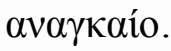

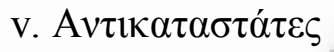

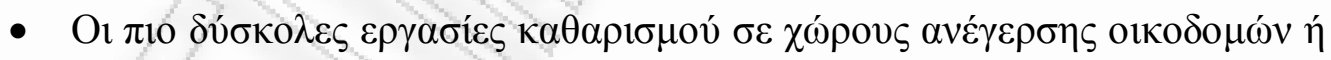

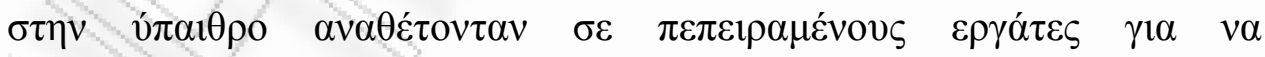

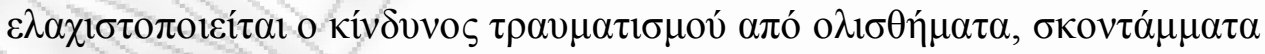

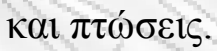

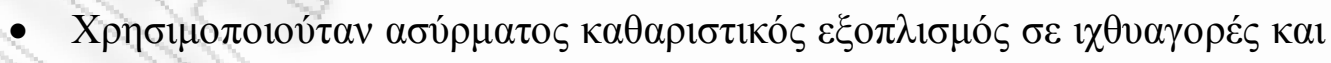

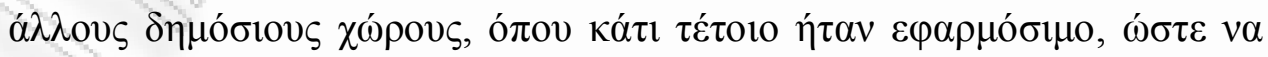

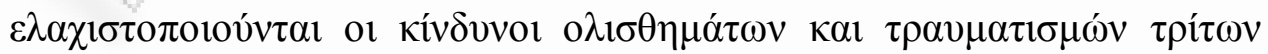
$\pi \rho \circ \sigma \omega ́ \pi \omega v$. 


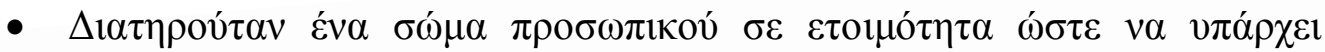

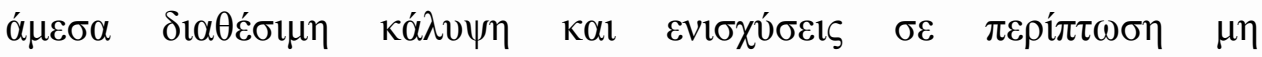

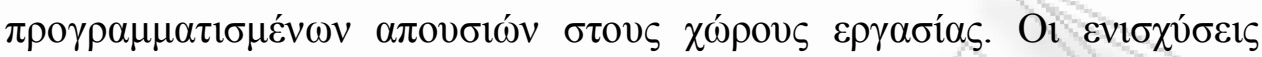

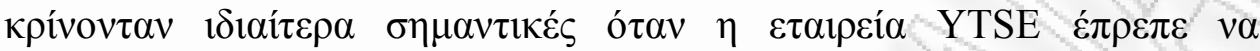

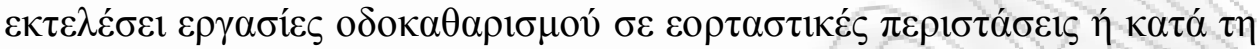
$\delta 1 \alpha ́ \rho \kappa \varepsilon 1 \alpha \delta 1 \alpha \delta \eta \lambda \omega ́ \sigma \varepsilon \omega v$.

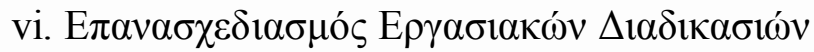

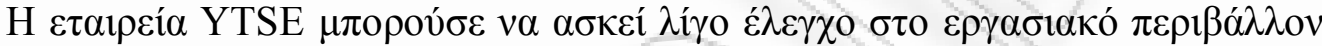

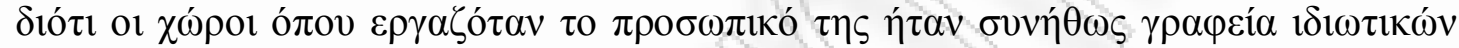

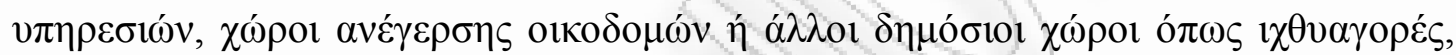

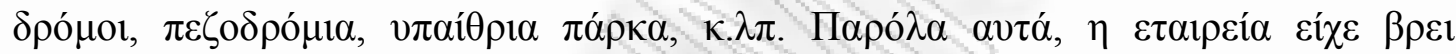

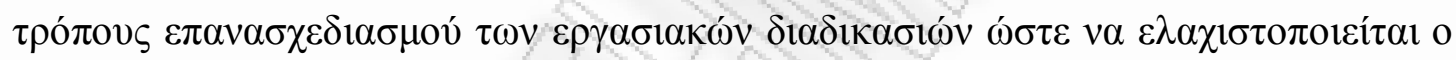

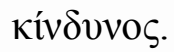

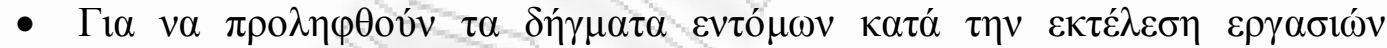

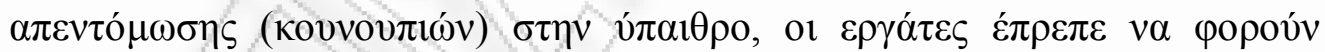

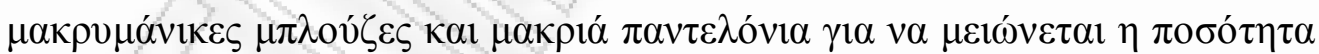

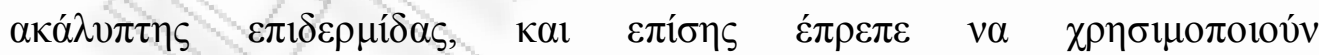

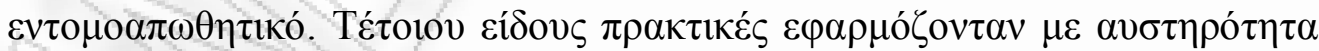

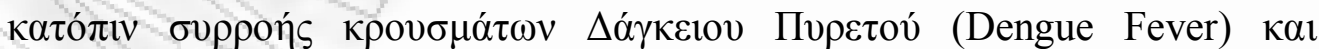

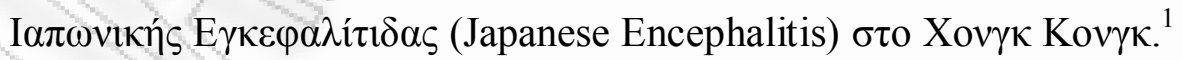

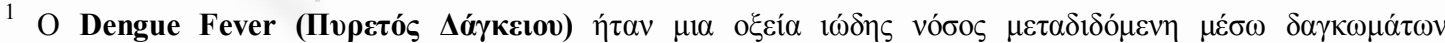

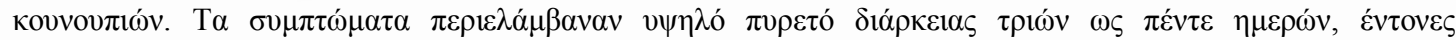

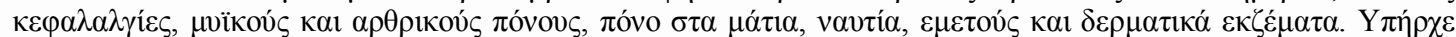

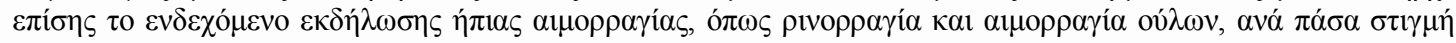

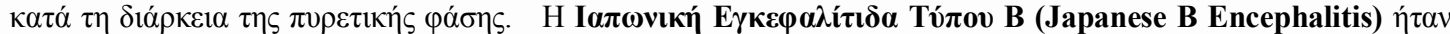

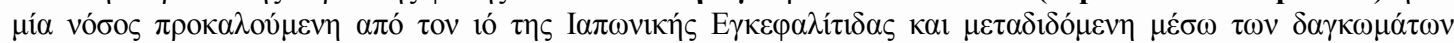

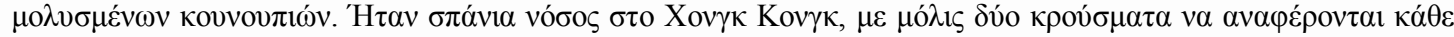

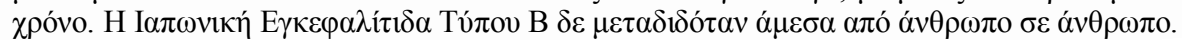




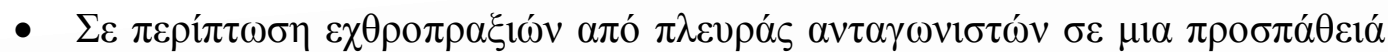

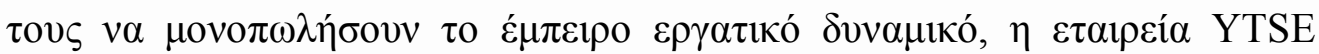

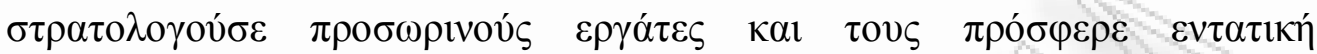

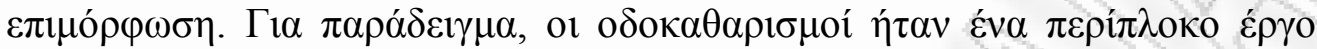

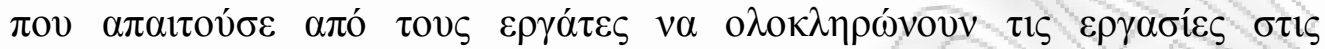

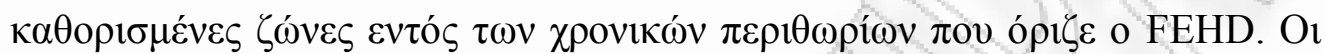

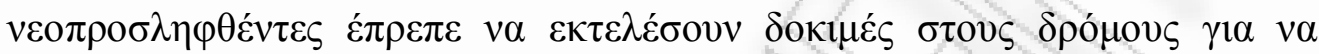

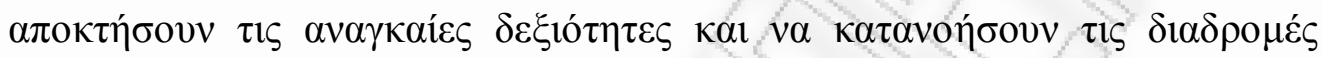

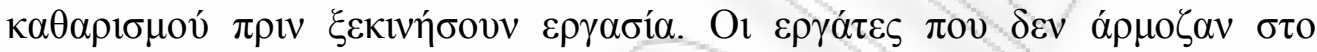

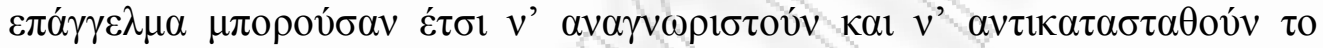

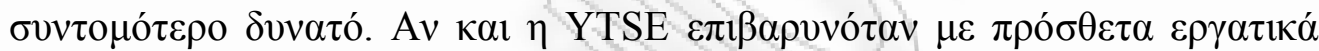

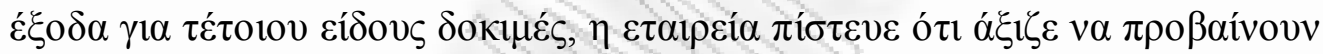

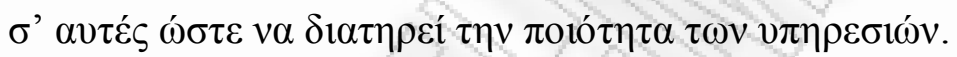

vii. Y $\pi \varepsilon v \theta v v o ́ \tau \eta \tau \alpha$

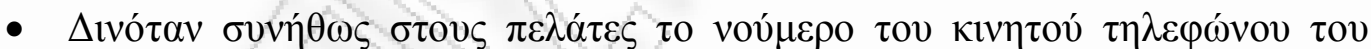

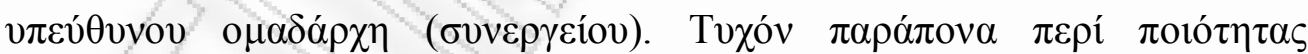

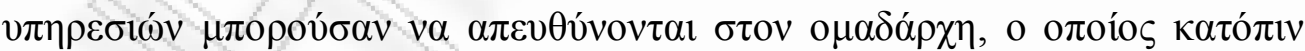

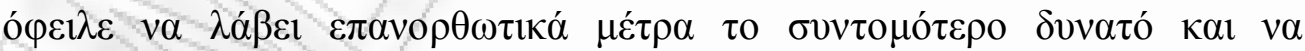

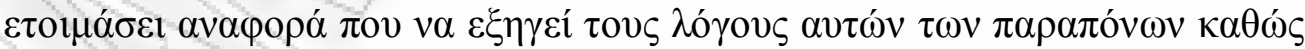

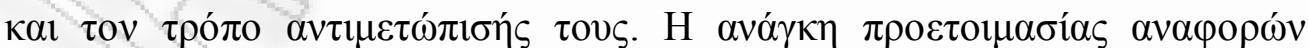

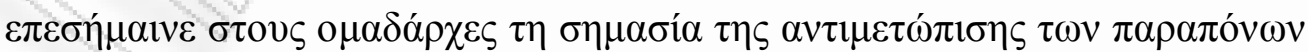

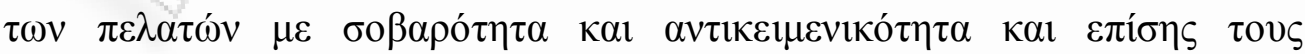

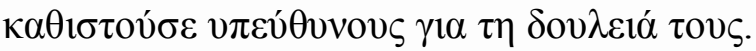




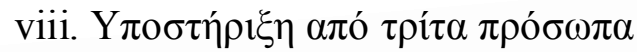

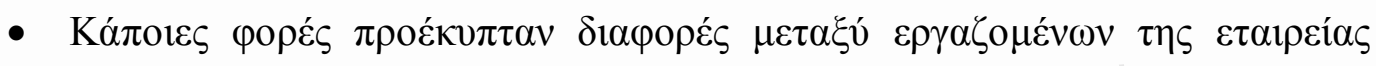

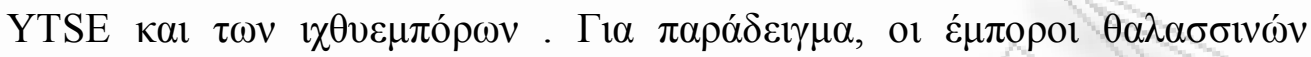

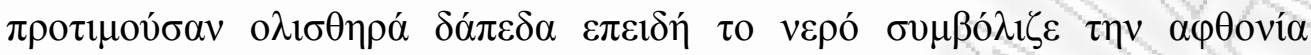

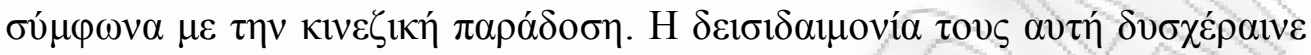

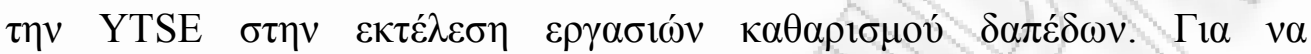

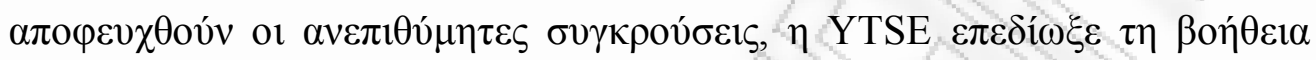

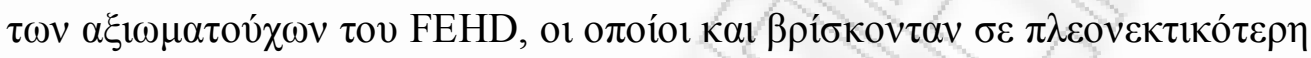

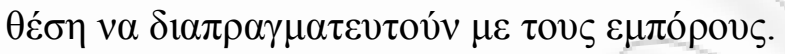

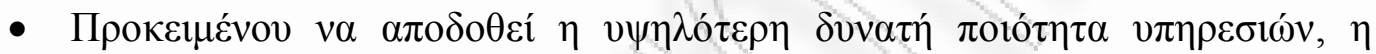

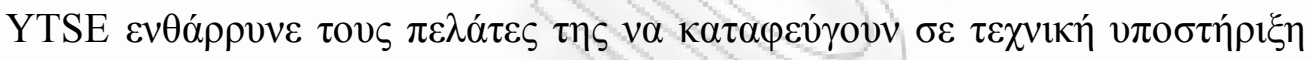

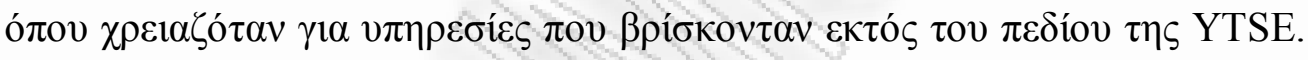

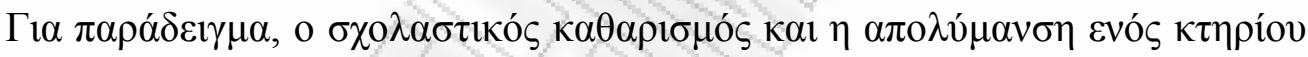

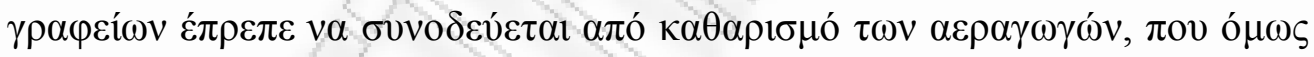

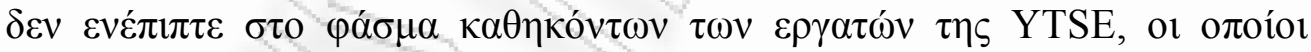

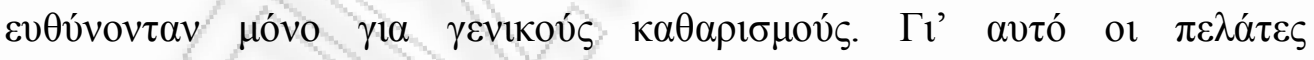

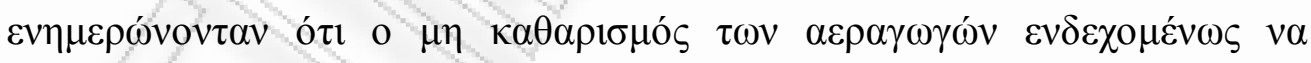

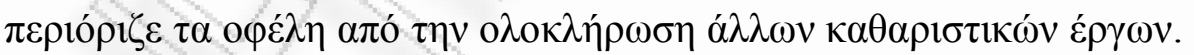

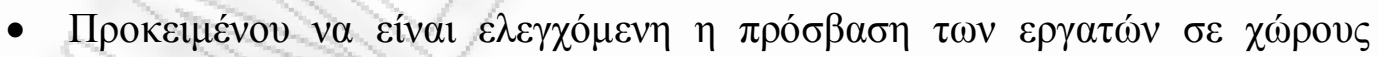

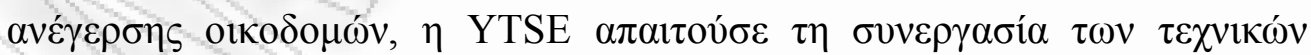

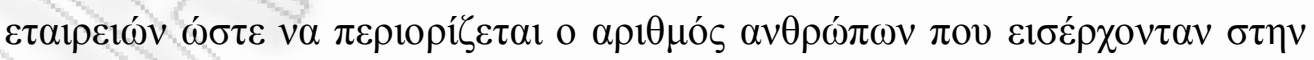

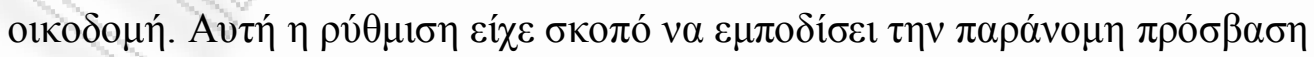

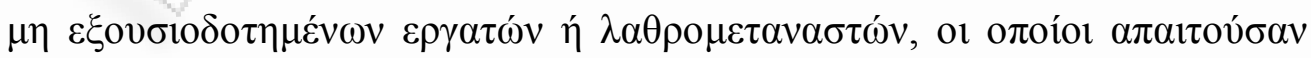

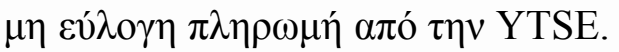




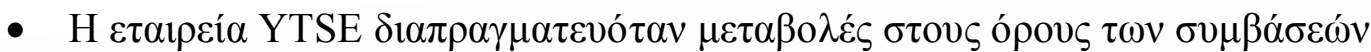

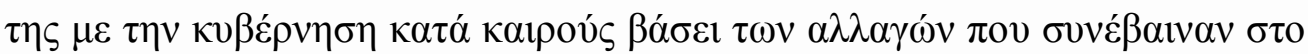

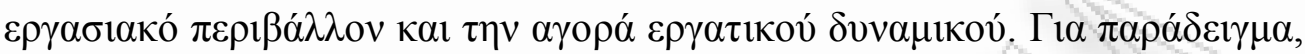

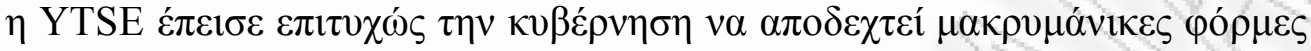

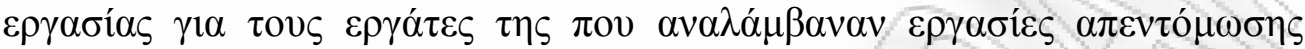

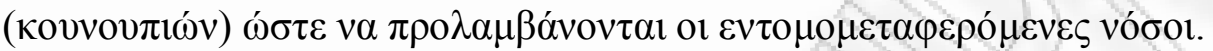

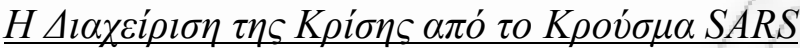

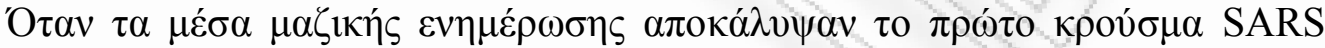

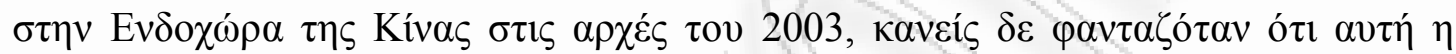

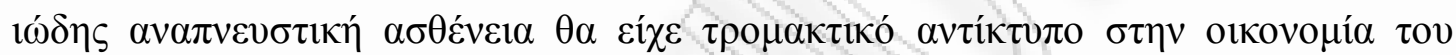

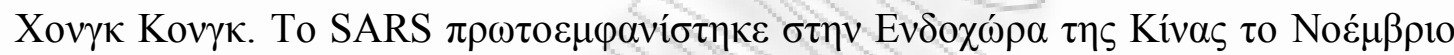

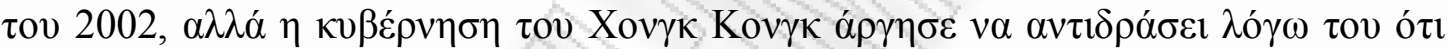

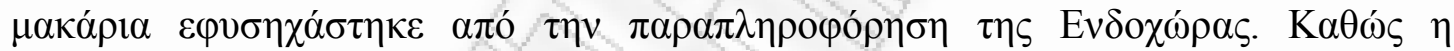

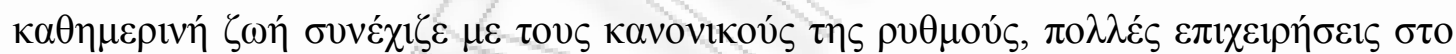

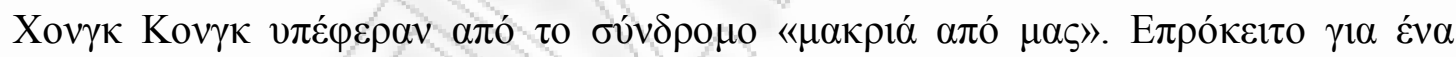

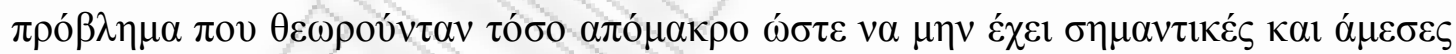

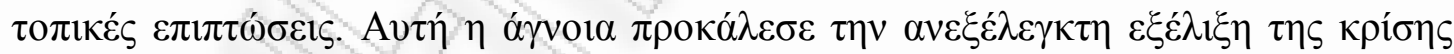

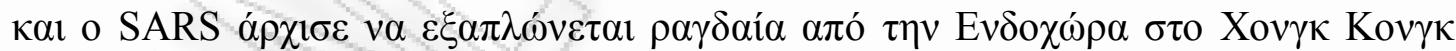

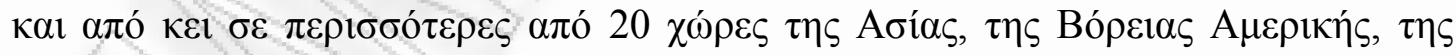

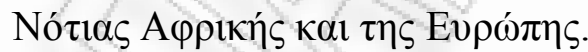

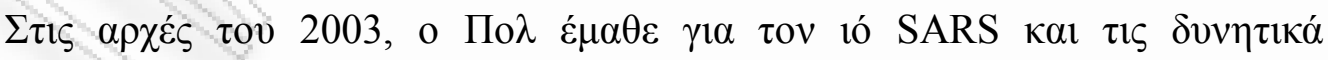

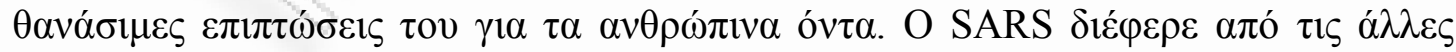

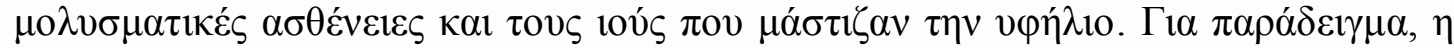

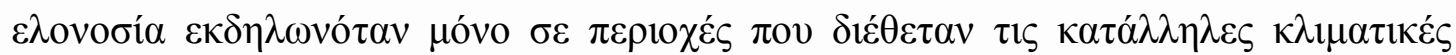




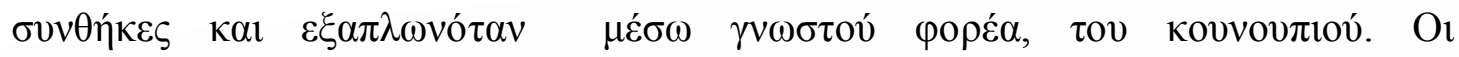

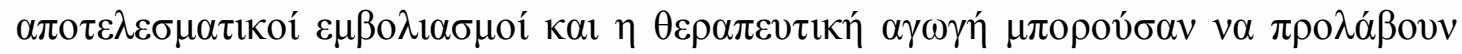

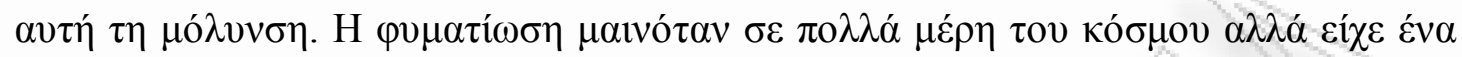

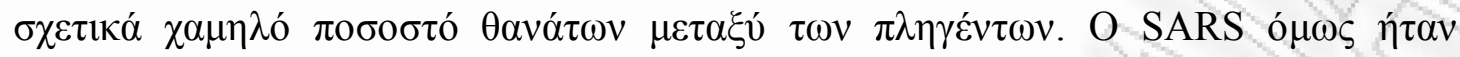

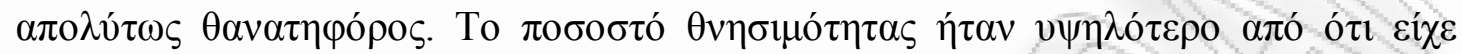

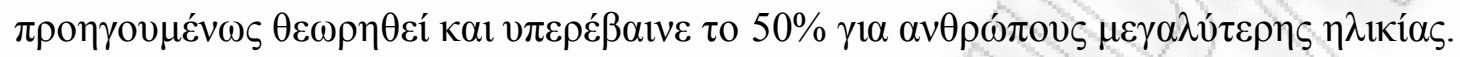

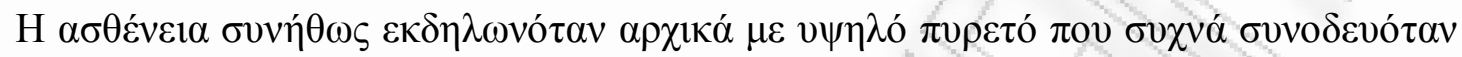

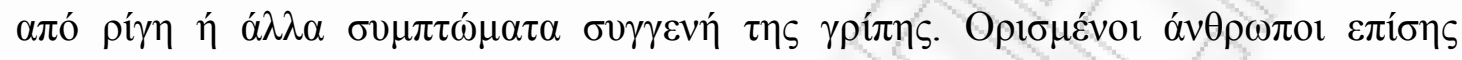

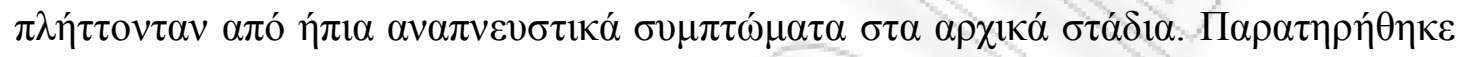

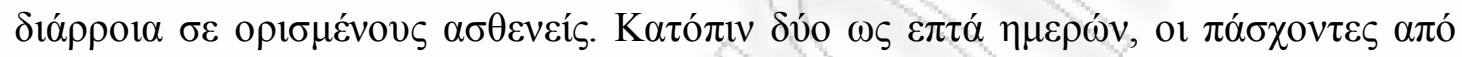

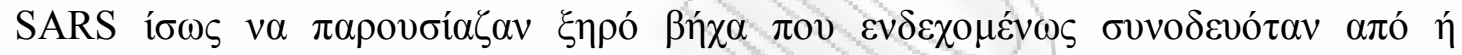

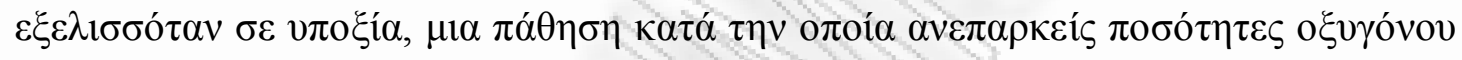

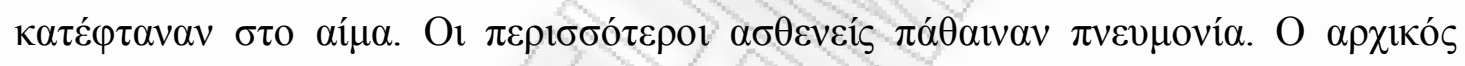

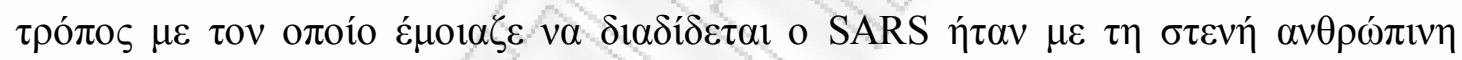

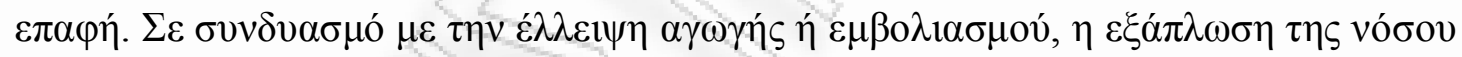

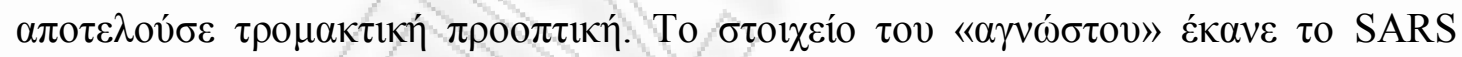

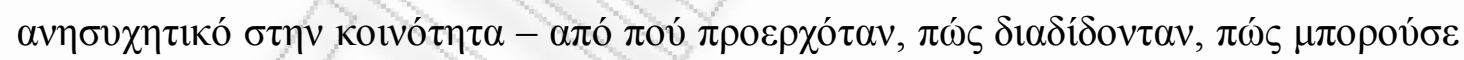

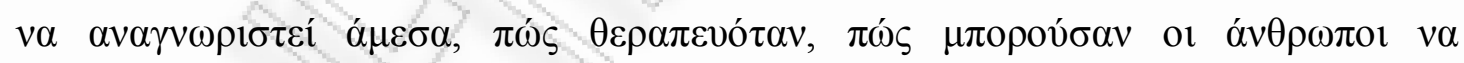

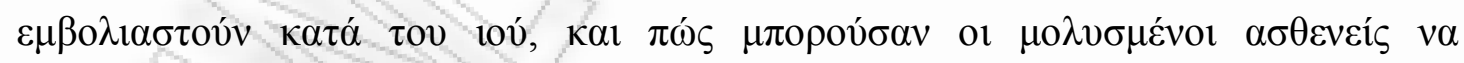
$\alpha \pi \circ \theta \varepsilon \rho \alpha \pi \varepsilon v \tau o u ́ v$

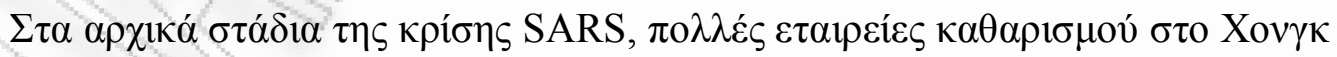

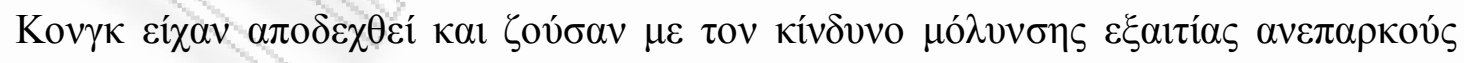

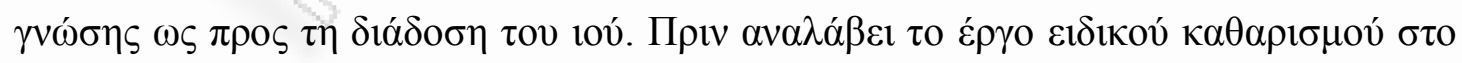

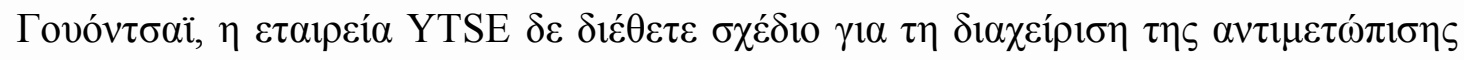

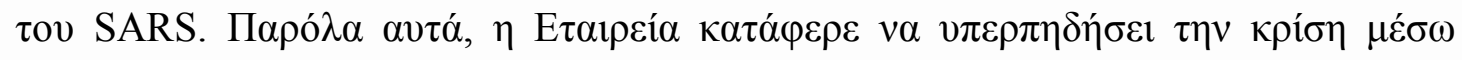




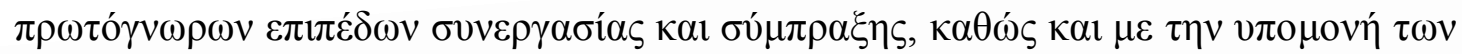

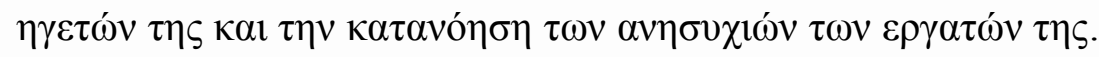

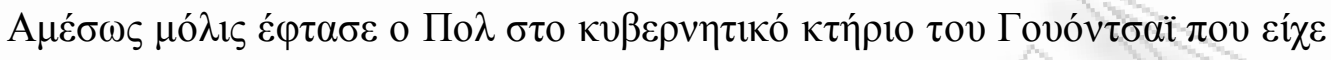

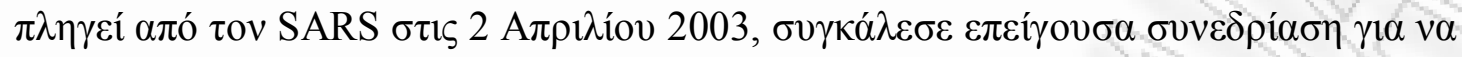

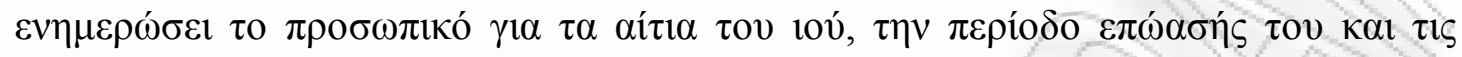

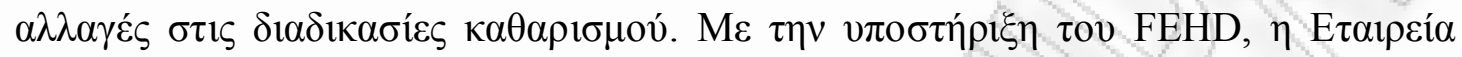

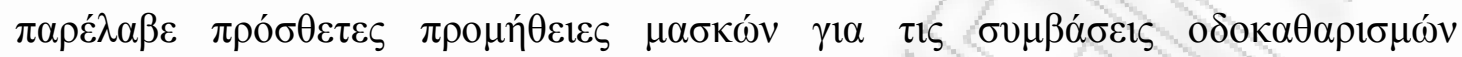

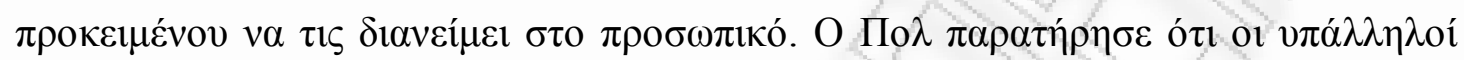

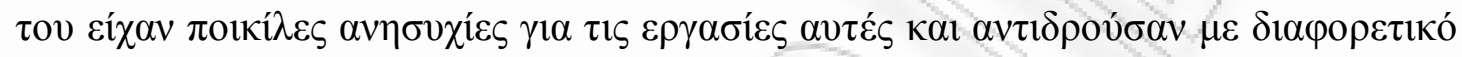

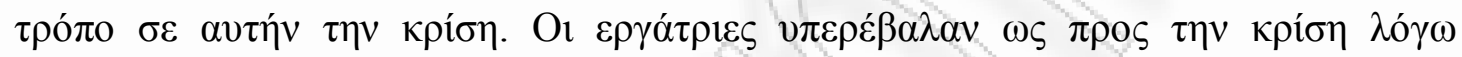

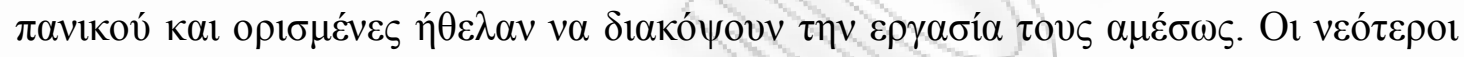

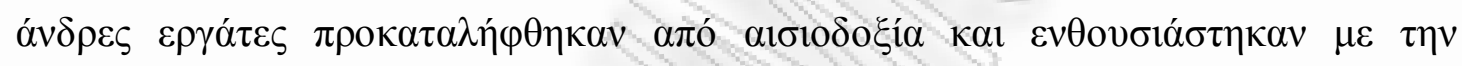

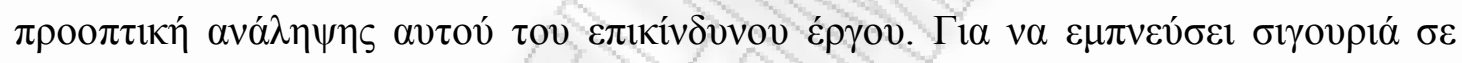

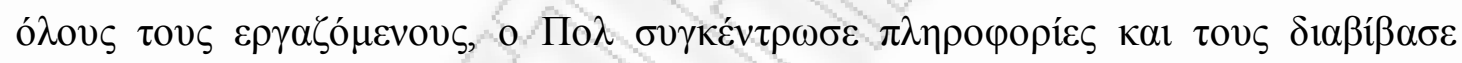

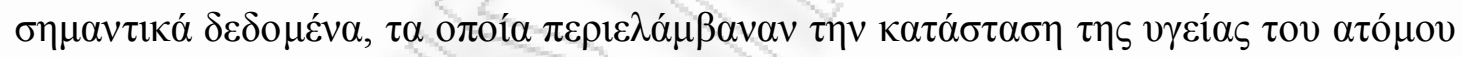

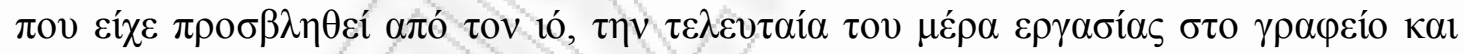

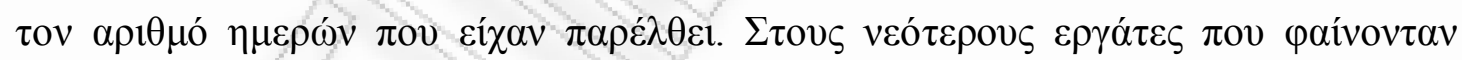

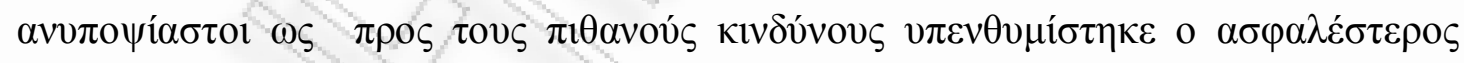

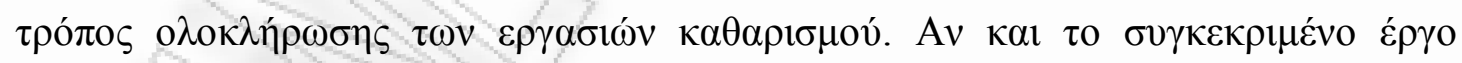

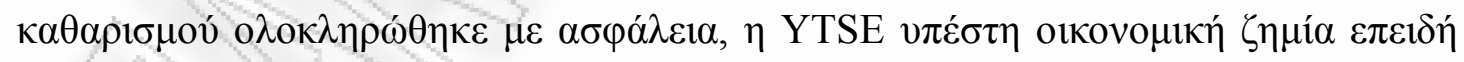

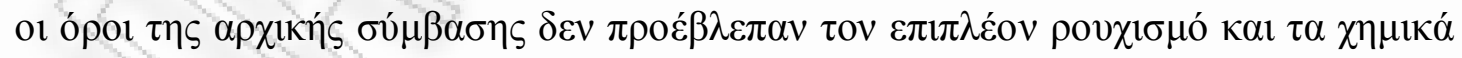

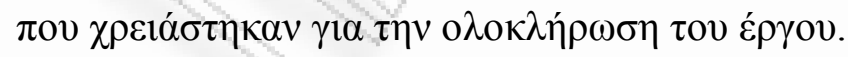

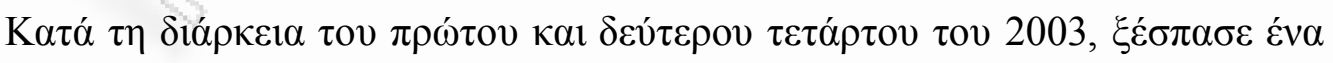

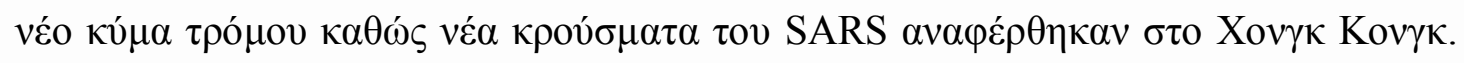

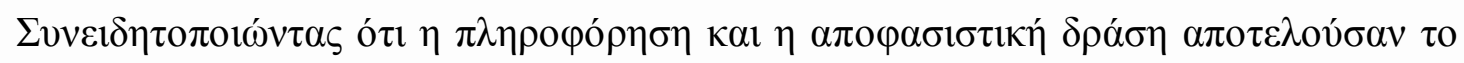




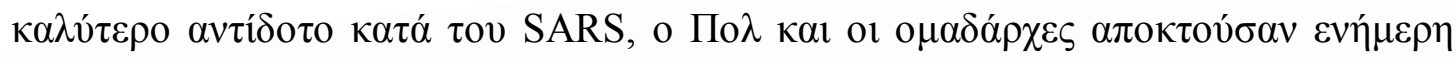

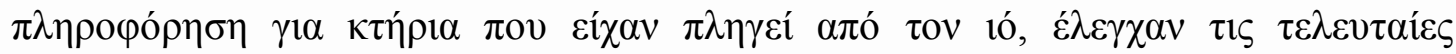

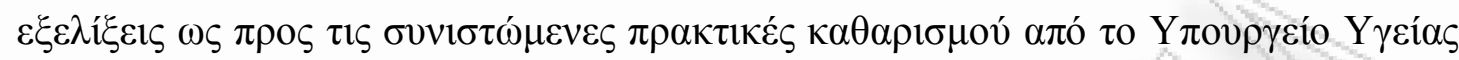

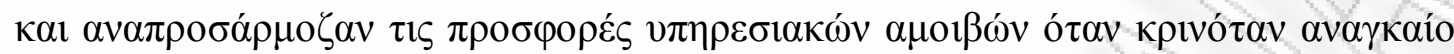

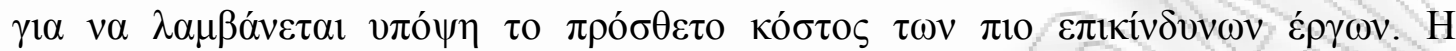

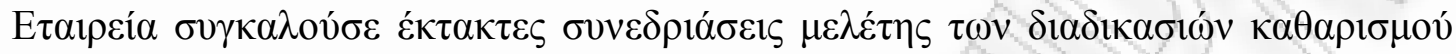

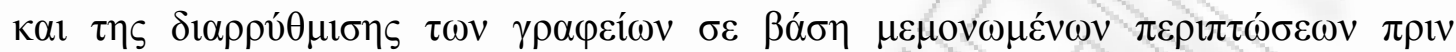

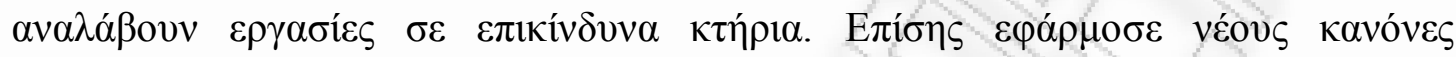

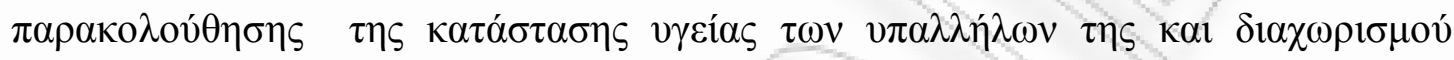

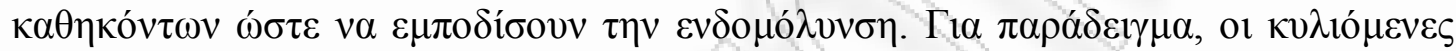

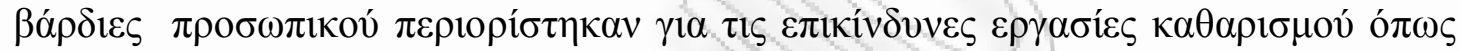

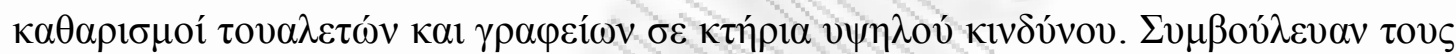

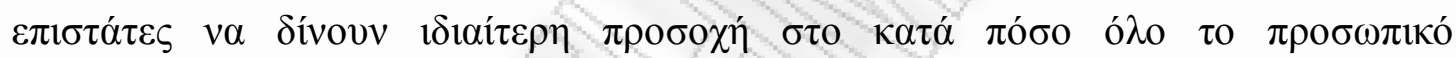

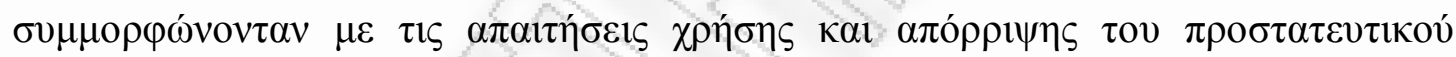

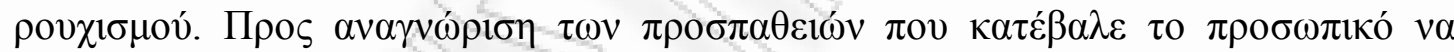

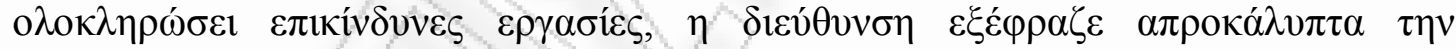

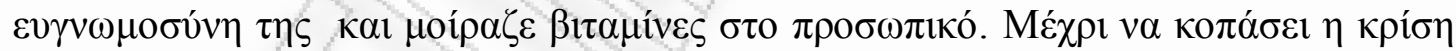

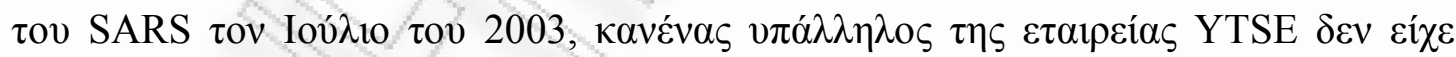
$\mu \mathrm{o} \lambda v v \theta \varepsilon i ́$

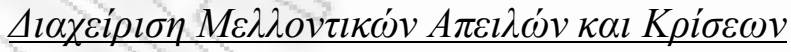

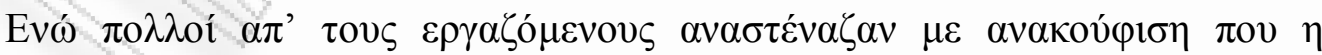

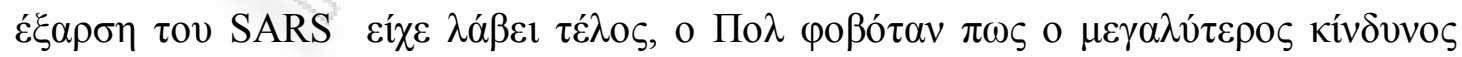

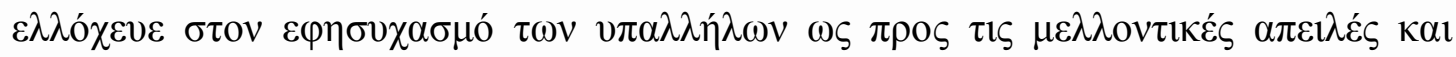

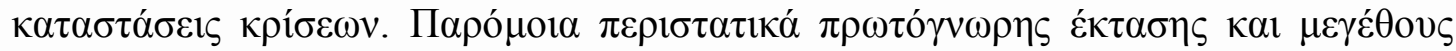




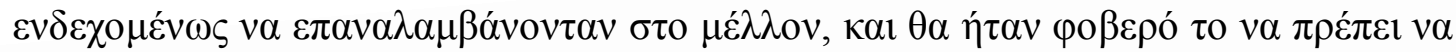

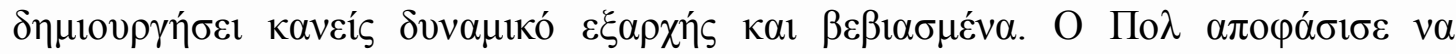

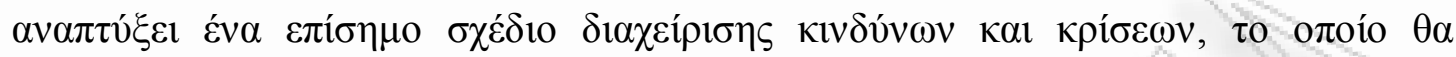

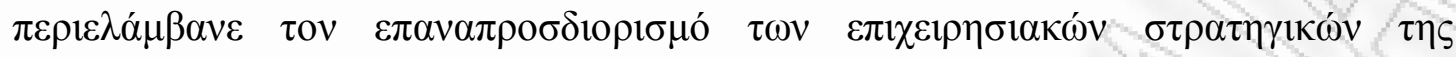

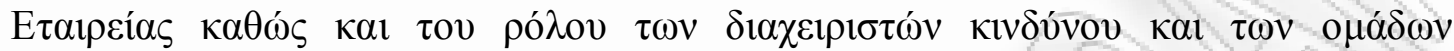

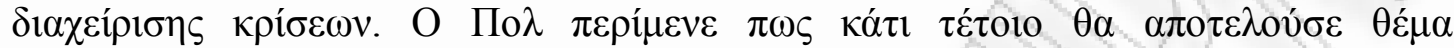

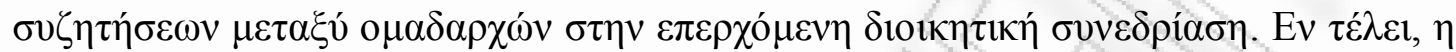

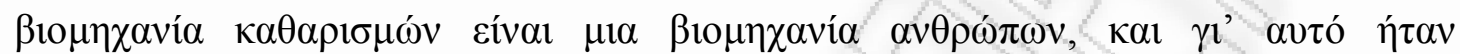

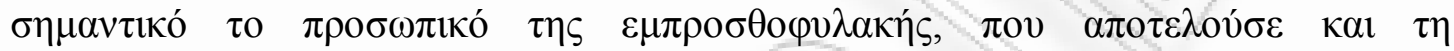

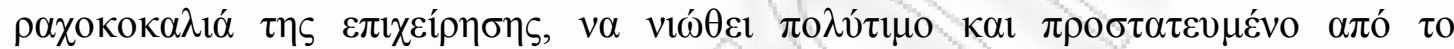

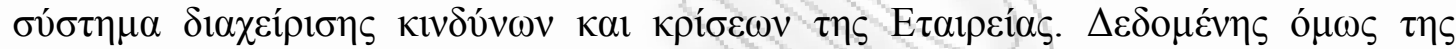

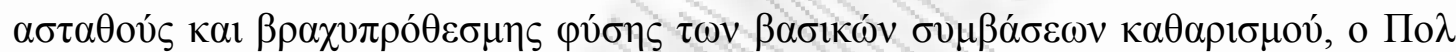

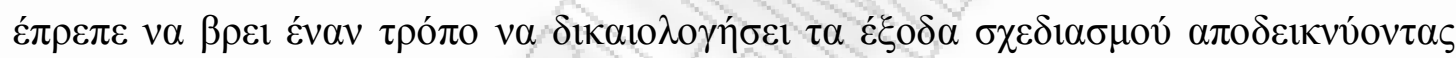

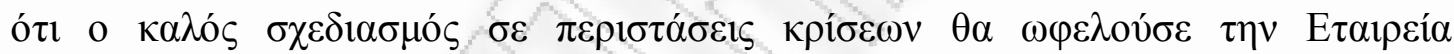
$\mu \alpha \kappa \rho о \pi \rho o ́ \theta \varepsilon \sigma \mu \alpha$. 


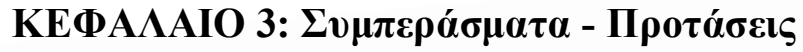

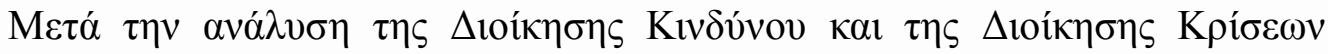

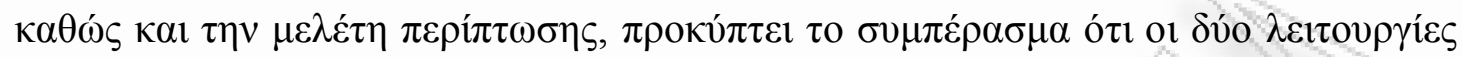

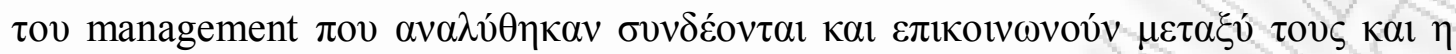

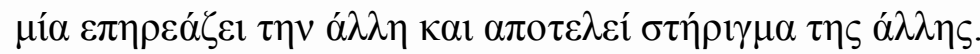

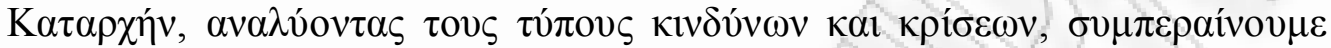

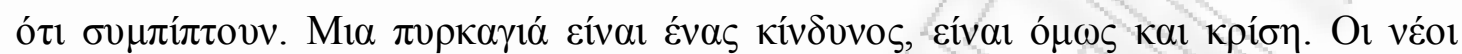

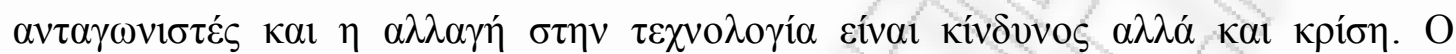

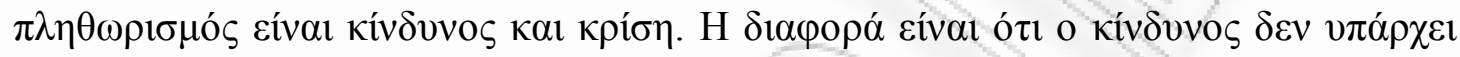

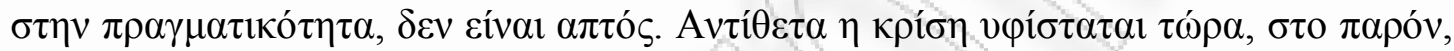

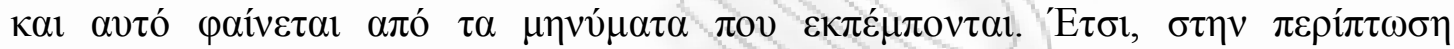

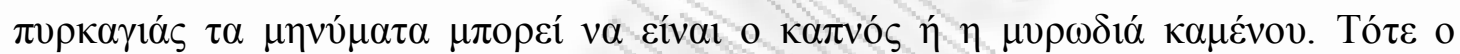

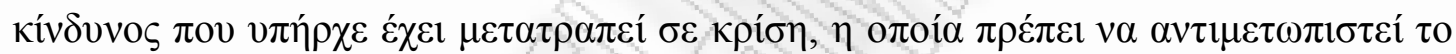

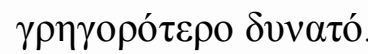

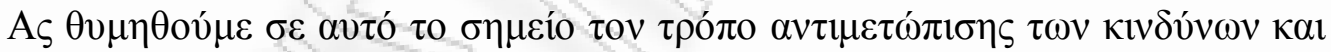

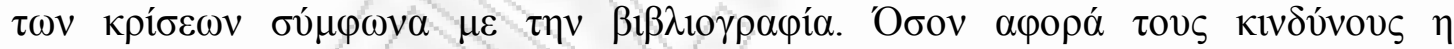

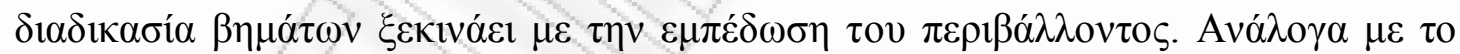

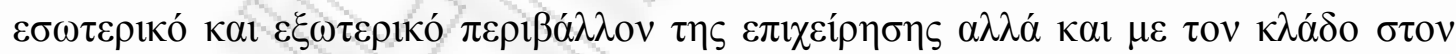

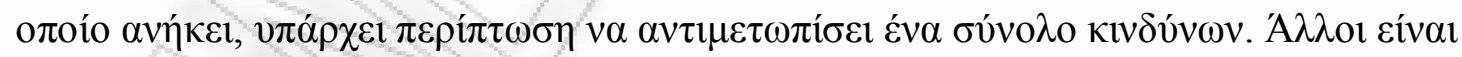

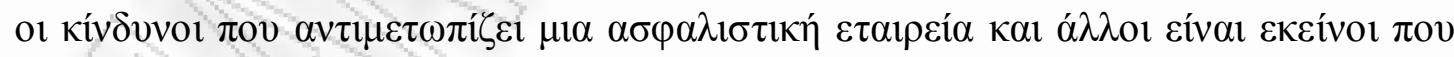

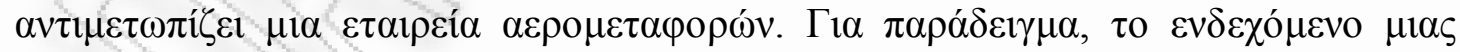

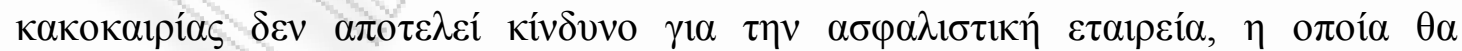

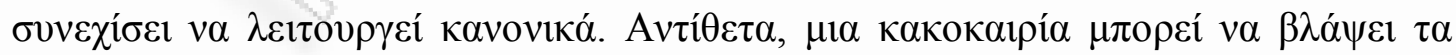

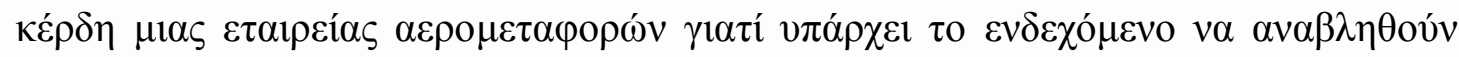




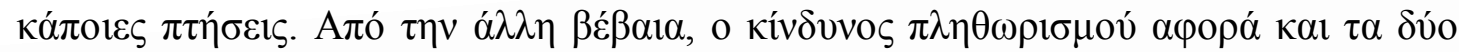

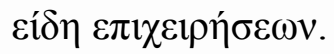

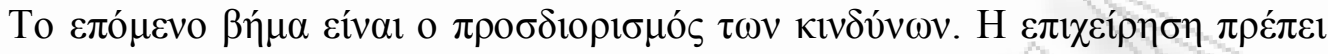

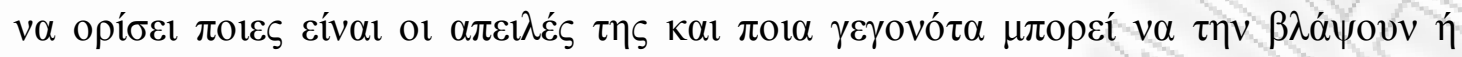

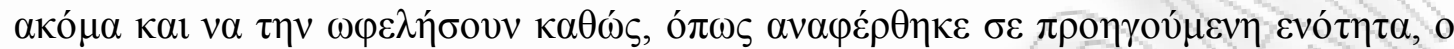

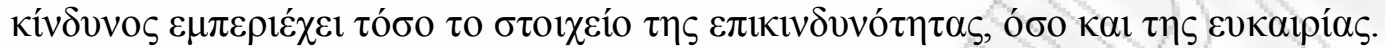

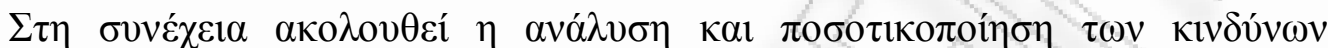

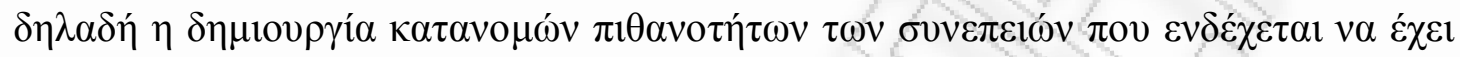

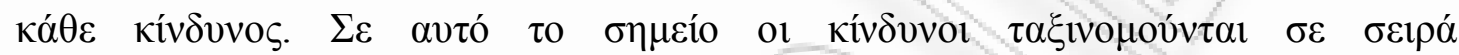

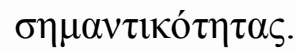

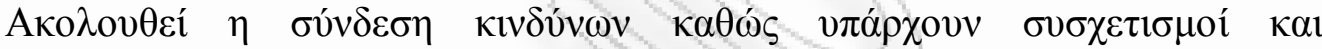

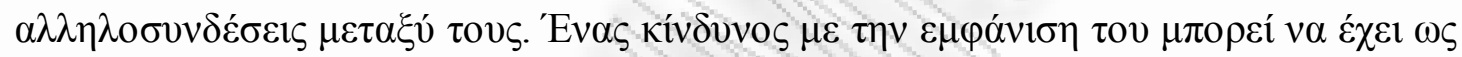

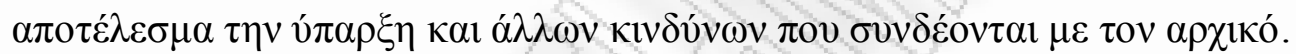

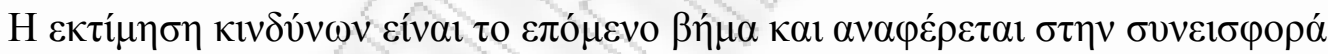

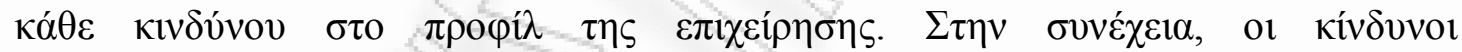

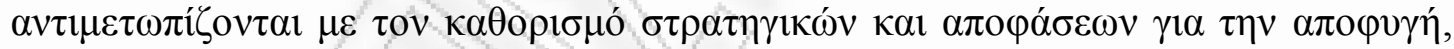

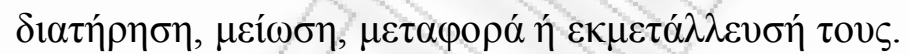

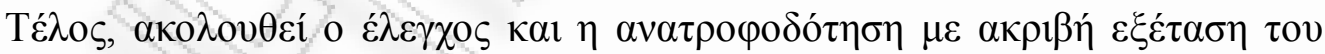

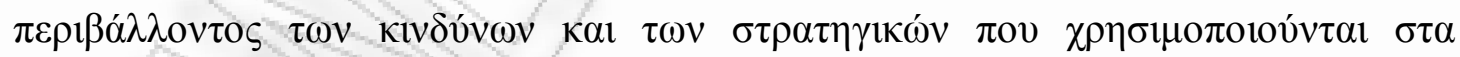

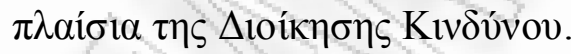

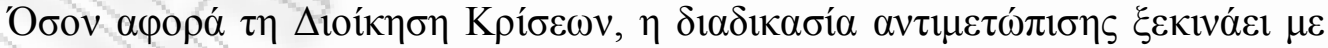

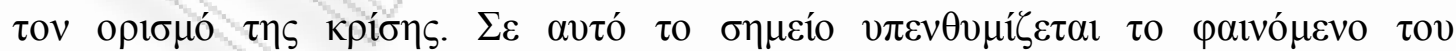

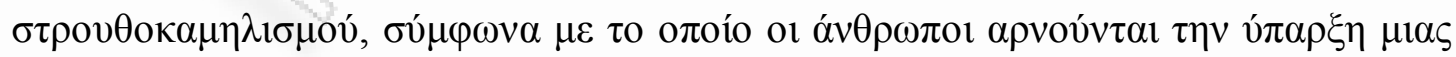

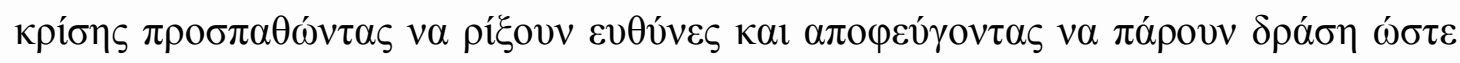

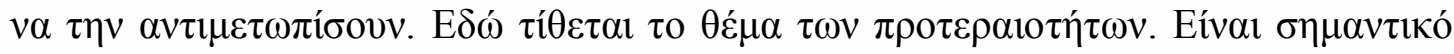




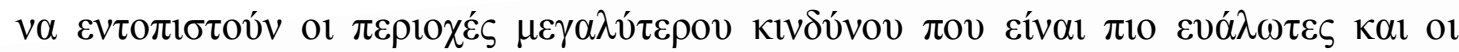

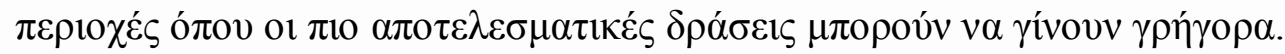

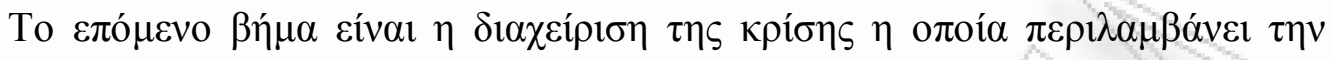

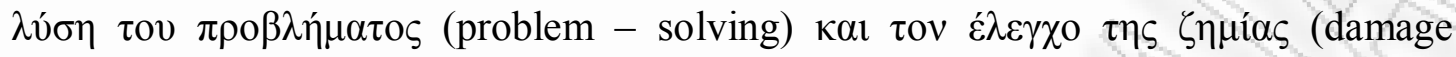

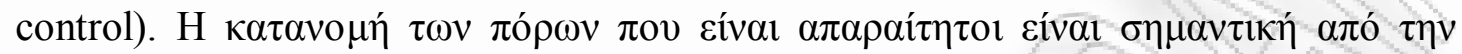

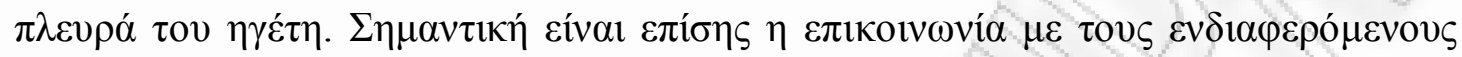

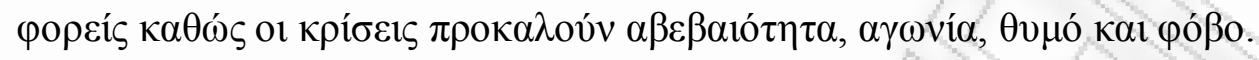

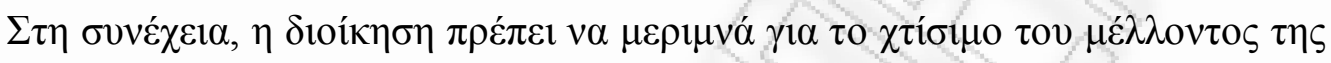

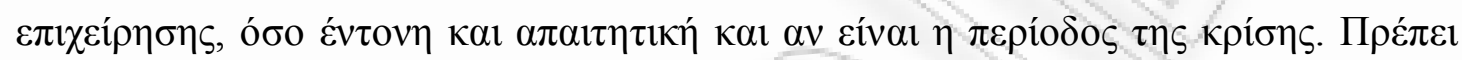

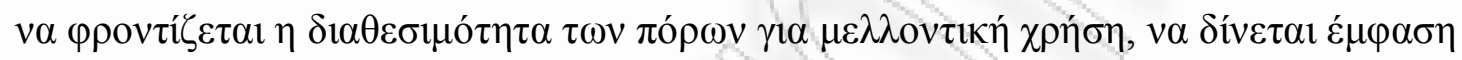

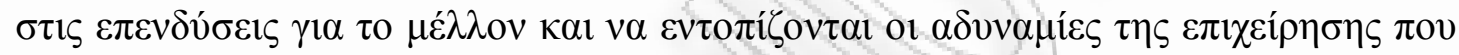

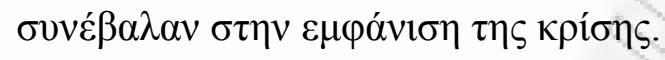

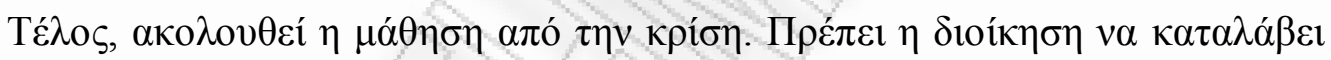

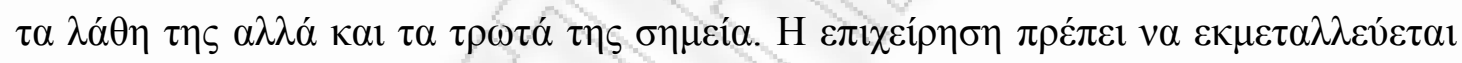

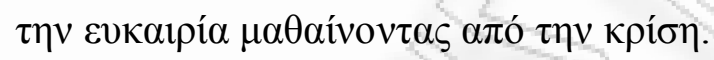

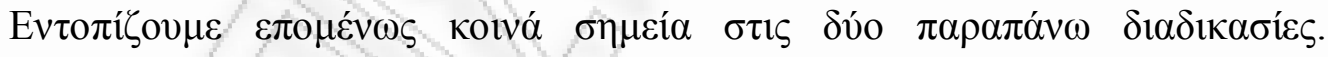

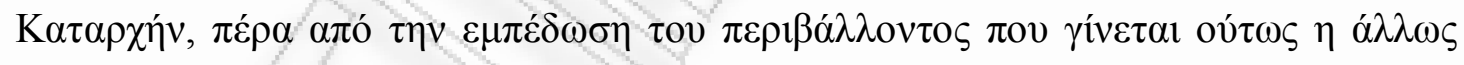

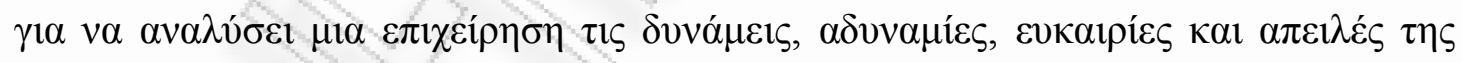

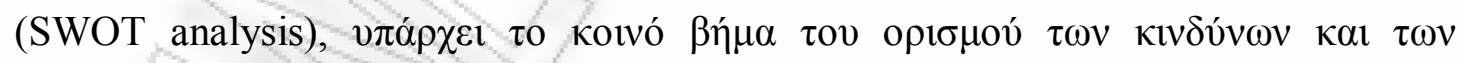

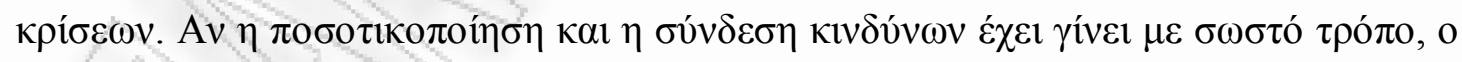

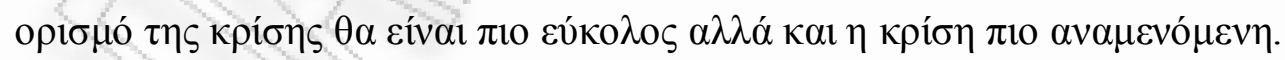

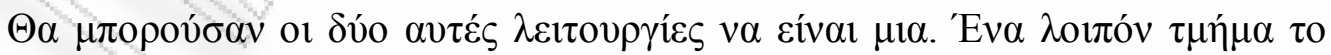

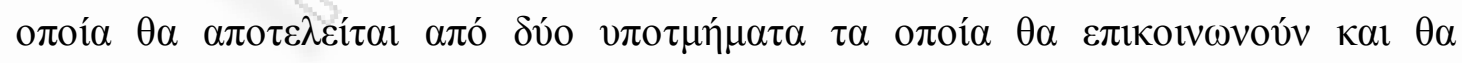

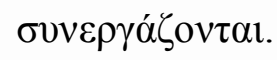




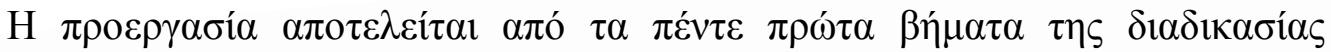

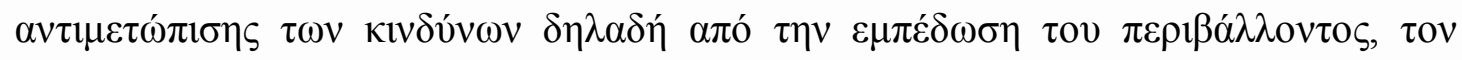

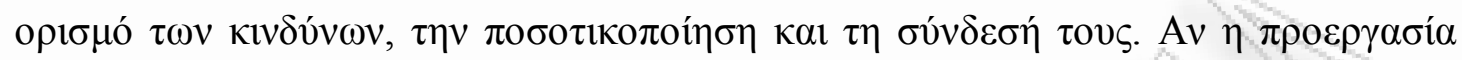

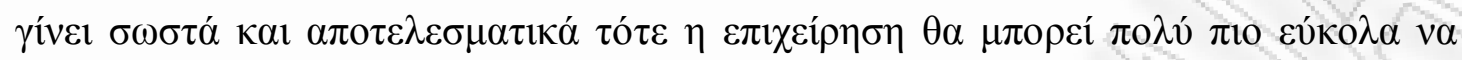

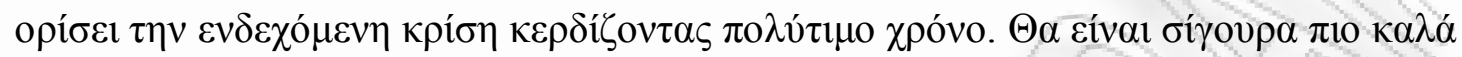

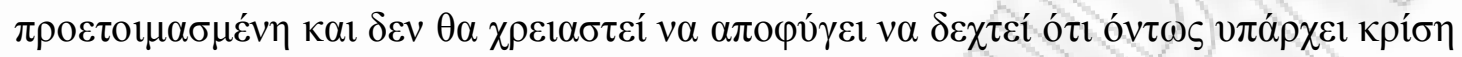

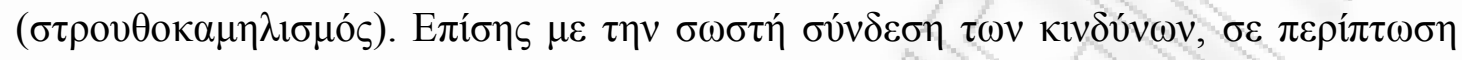

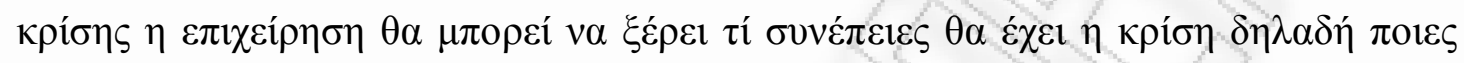

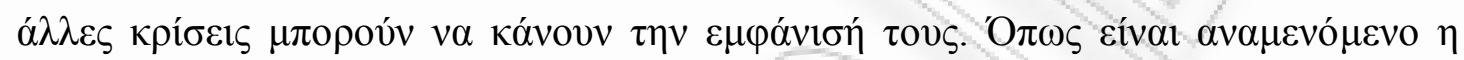

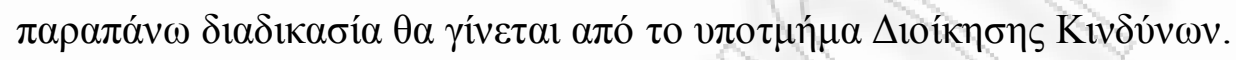

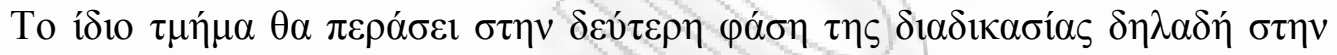

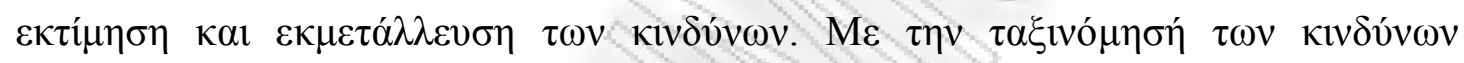

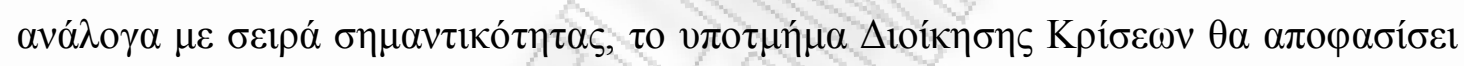

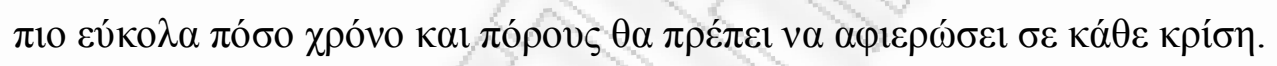

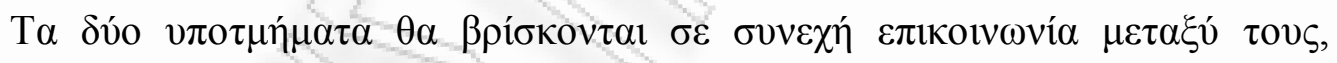

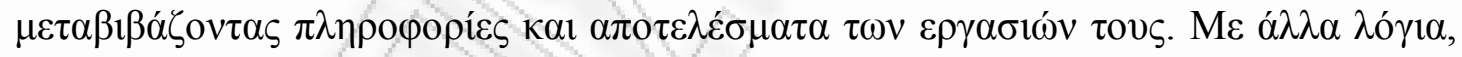

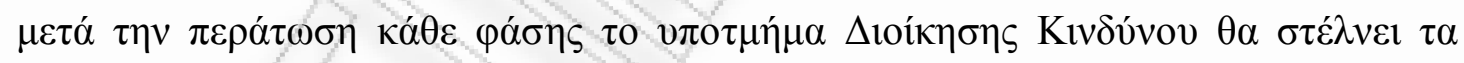

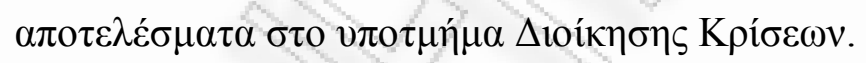

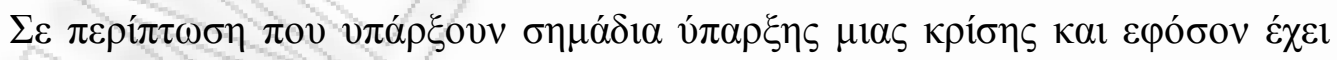

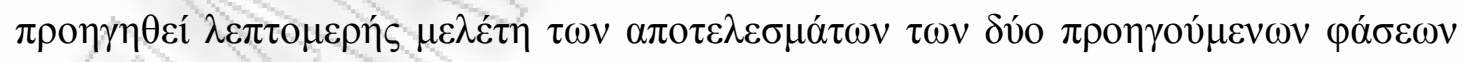

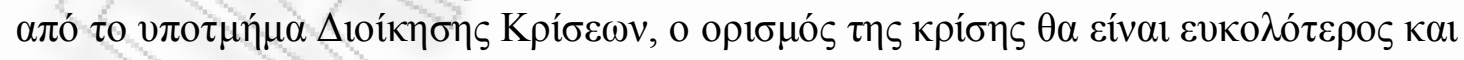

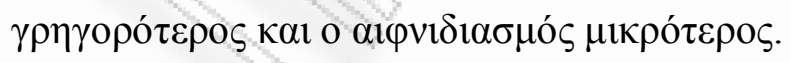

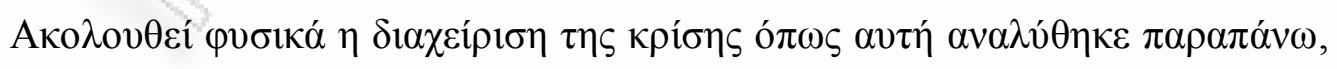

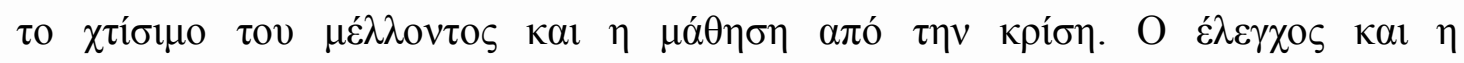




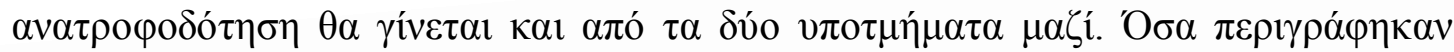

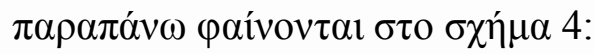

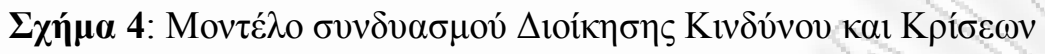

\begin{tabular}{|c|c|c|}
\hline BHMA $1^{\circ}$ & $B H M A 2^{\circ}$ & BHMA $3^{O}$ \\
\hline 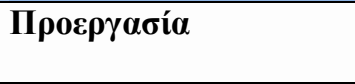 & 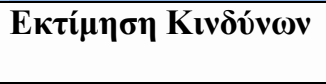 & 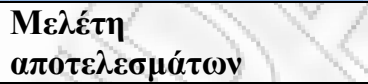 \\
\hline 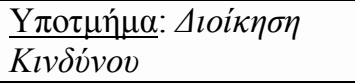 & 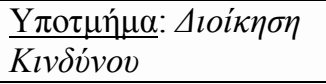 & 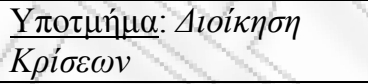 \\
\hline 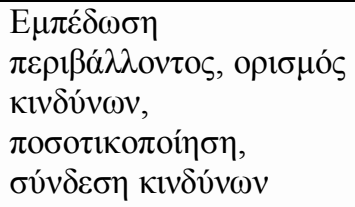 & 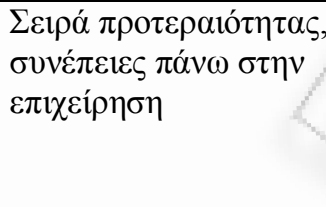 & 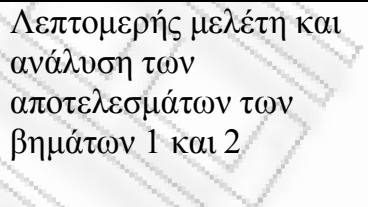 \\
\hline
\end{tabular}

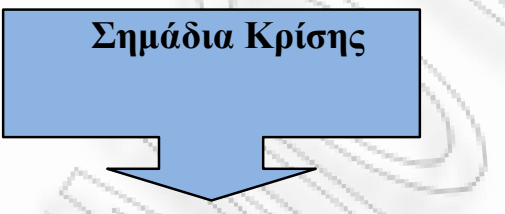

\begin{tabular}{|c|c|c|}
\hline$\underline{B H M A} 4^{\circ}$ & $\underline{B H M A 5^{\circ}}$ & $B H M A 6^{\circ}$ \\
\hline 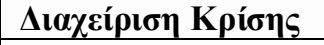 & 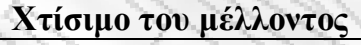 & 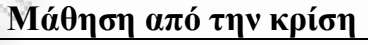 \\
\hline 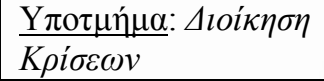 & 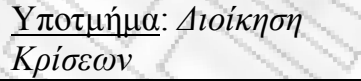 & 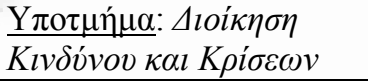 \\
\hline 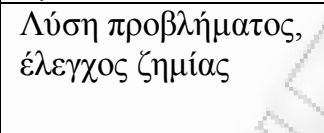 & 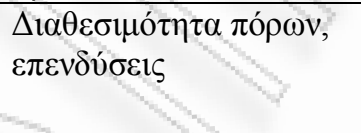 & 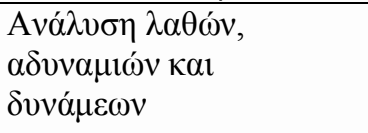 \\
\hline
\end{tabular}

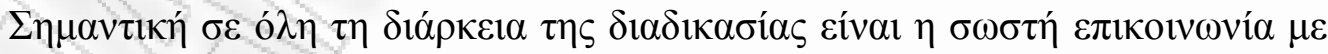

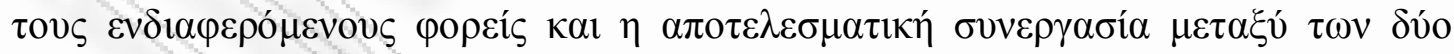
$v \pi 0 \tau \mu \eta \mu \alpha ́ \tau \omega v$.

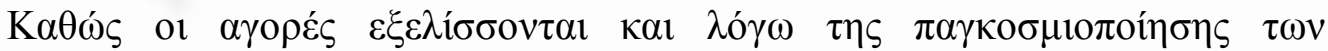

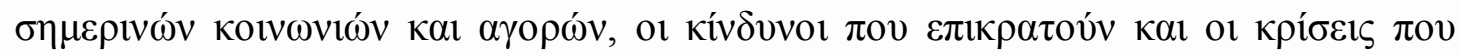

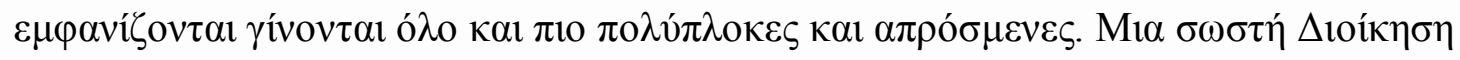




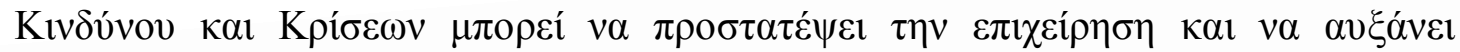

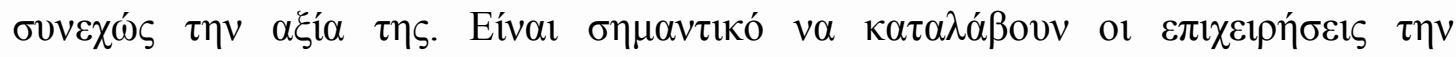

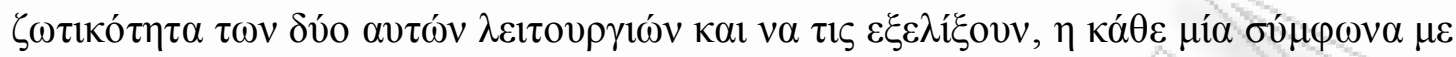
$\tau 1 \varsigma \delta 1 \kappa \varepsilon ́ \varsigma \tau \eta \varsigma \alpha \nu \alpha ́ \gamma \kappa \varepsilon \varsigma$. 


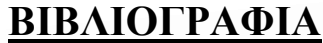

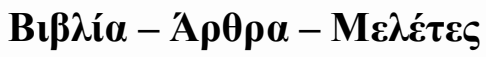

- Suzanne Labarge, "Chief Risk Officers: Should Your Organization Have One?", 2002

- Lisa K. Meulbroek, "Integrated Risk Management for the Firm: A Senior Manager's Guide”, 2002

- James Lam, "Enterprise Risk Management. From Incentives to Controls" , 2003

- Hull J.C., "Options, Futures and Other Derivatives", 2000

- Young, Peter C. and Tippins, Steven C., "Managing Business Risk: An Organization Wide Approach to Risk Management', 2000

- Douglas McLeod, "New Chief Risk Officer Role Coordinates Risk Strategy", 1999

- Brian W. Nocco and Rene M. Stulz, "Enterprise Risk Management: Theory and Practice", 2006

- $\quad$ Rosabeth Moss Kanter, "Note on Management of Crisis", 1996

- Fink Steven, "Crisis Management: Planning for the Inevitable”, 1986

- Selbst P., “The Containment and Control of Organizational Crises”, 1978

- Davies H. and Walters M," "Do all crises have to become disasters? Risk and risk mitigation", 1998

- Harvard ManageMentor, "Managing a Crisis. How to make good decisions in the heat of the moment", 2005

- Michael Watkins, “Assessing Your Organization's Crisis Response Plans”, 2001

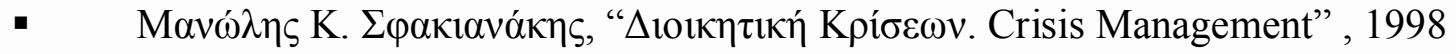




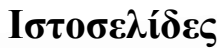

- $\quad$ www.investorwords.com

- $\quad$ www.ferma.eu

- $\quad$ www.standardas.org.au

- $\quad$ www.det.act.gov.au

- $\quad$ www.findarticles.com

- $\quad$ www.casact.org

- $\quad$ www.towersperrin.com

- $\quad$ www.tillinghast.com

- $\quad$ www.crisismanagement-disasterrecovery.com

- $\quad$ www.crisisexperts.com

- $\quad$ www.wikipedia.com

- www.kathimerini.gr

- $\quad$ www.israelnewsagency.com

- $\quad$ http://biznewsletter.wordpress.com 